\title{
Multiple Imputation of Missing Data in Multilevel Designs: A Comparison of Different Strategies
}

\author{
Oliver Lüdtke ${ }^{1,2}$, Alexander Robitzsch ${ }^{1,2}$ and Simon Grund ${ }^{1,2}$ \\ ${ }^{1}$ Leibniz Institute for Science and Mathematics Education, Kiel, Germany \\ ${ }^{2}$ Centre for International Student Assessment, Germany
}

Supplemental Online Material 
Enclosed in this document are the supplemental materials for our article entitled "Multiple Imputation of Missing Data in Multilevel Designs: A Comparison of Different Strategies". Supplement A contains the results of an additional simulation study that featured a multilevel model with random slopes as the data generating model. Supplement B contains the results of an additional simulation study that compared the procedures discussed in the main article under a data generating model with either manifest or latent group means. Supplement $\mathrm{C}$ contains the computer code that was used to calculate the data analysis example using the different missing data methods. Supplement D contains additional tables to the results reported in the article.

\section{Supplement A: Simulation Results for Multilevel Model with Random Slopes}

We conducted an additional simulation study in which we investigated the performance of the PAN approach for a multilevel model with random slopes:

$$
Y_{i j}=\mu_{Y}+\beta_{W, Y X} X_{W, i j}+\beta_{B, Y X} X_{B, j}+v_{0 j}+v_{1 j} X_{W, i j}+\varepsilon_{i j}
$$

where $\beta_{W, Y X}$ and $\beta_{B, Y X}$ are the within- and between-group regression coefficients; $v_{0 j}$ and $v_{1 j}$ are normally distributed random effects with mean zero and variances $\tau_{0}^{2}$ and $\tau_{1}^{2}$, and covariance $\tau_{01}$. Note that the between part of the level-1 predictor is treated as a latent variable (see also model in Equation 6 in the main article). Preacher, Zhang, and Zyphur (in press) provide a detailed discussion of random slope models with latent between parts of level-1 variables.

We used selected conditions of the simulation in the main article and simulated $K=150$ groups of medium size $(n=15)$, where the ICCs for $X$ and $Y$ were constrained to be equal and set to .10 or .30 . The correlation within groups $\left(\rho_{W}\right)$ was set to .35 , and the correlation between groups $\left(\rho_{B}\right)$ to .60. For simplicity's sake, the random effects were assumed to be independent of one another $\left(\tau_{01}=0\right)$, and the slope variance $\left(\tau_{1}^{2}\right)$ was varied with values 
of $.01, .05, .10$, and .20 . Missing data (deleting 25\%) in either $Y$ or $X$ were induced by an MCAR or MAR mechanism by setting $\lambda$ to 0 or .8 (see Equation 29 in the main article).

In addition to the PAN approach, which is based on a joint modeling approach, we also used a conditional model for imputing the missing data. In the case of missing values on $Y$, for instance, a multilevel model with random slopes is used in which $Y$ is the dependent and $X$ is the predictor variable. This approach was suggested for handling incomplete variables in models with random slopes (see Enders, Mistler, \& Keller, in press). It was implemented in a chained equations framework (also known as "fully conditional specification", FCS) using the function mice. impute. 21. pan in the R package mice (van Buuren \& Groothuis-Oudshoorn, 2011). To accommodate group-level effects of level1 predictors in the imputation model, the observed group means were also included as additional predictors in the FCS approach (see Enders et al., in press). We used Mplus 7.3 to estimate the multilevel model with random slopes. Note that Mplus uses full information maximum likelihood estimation, which may result in slightly biased estimates of variance components. Thus, we included the results obtained from complete data sets as a benchmark.

The main questions were (a) whether the PAN approach provides valid estimators of $\beta_{W, Y X}$ and $\beta_{B, Y X}$ despite ignoring the slope variation, and (b) whether the FCS approach provides an improved estimation of the slope variance $\left(\tau_{1}^{2}\right)$ compared to the PAN approach. Table 1 presents the main results for the bias and the coverage rates of the $95 \%$ confidence intervals when the slope variance is large $\left(\tau_{1}^{2}=.20\right)$.

First, in the case where the dependent variable is incomplete $(Y \sim X)$, both the PAN approach and the FCS approach produced approximately unbiased estimates of the withingroup and between-group regression coefficient. For the variance components, the estimators provided by the FCS approach were very close to those obtained from the complete data sets, whereas the PAN approach underestimated the slope variance. It is also worth noting that the coverage rates produced by the PAN approach were lower than the nominal 
Table 1

Bias and Coverage of the 95\% Confidence Interval for the Estimators of the Fixed Effects and Variance Components in the Random Slope Model ( $Y$ on $X)$ for Large Intraclass Correlations $\left(\rho_{I, X}=\rho_{I, Y}=.30\right)$

\begin{tabular}{|c|c|c|c|c|c|c|c|c|c|c|c|c|}
\hline & \multicolumn{6}{|c|}{$\operatorname{MCAR}(\lambda=0)$} & \multicolumn{6}{|c|}{$\operatorname{MAR}(\lambda=0.8)$} \\
\hline & \multicolumn{3}{|c|}{ Bias } & \multicolumn{3}{|c|}{ Coverage $(95 \%)$} & \multicolumn{3}{|c|}{ Bias } & \multicolumn{3}{|c|}{ Coverage $(95 \%)$} \\
\hline & $\mathrm{CD}$ & PAN & FCS & $\mathrm{CD}$ & PAN & FCS & $\mathrm{CD}$ & PAN & FCS & $\mathrm{CD}$ & PAN & FCS \\
\hline & \multicolumn{12}{|c|}{ Missing $Y \sim X(25 \%)$} \\
\hline$\beta_{W, Y X}(.350)$ & .001 & .000 & .001 & .935 & .879 & .940 & .001 & .000 & .001 & .943 & .851 & .943 \\
\hline$\beta_{B, Y X}(.600)$ & -.005 & -.005 & -.005 & .952 & .951 & .951 & .000 & -.001 & .001 & .950 & .949 & .950 \\
\hline$(.192)$ & .025 & .005 & .028 & & & & .025 & .016 & .026 & & & \\
\hline \multirow[t]{2}{*}{$(.200)$} & -.021 & -.100 & -.019 & & & & -.022 & -.130 & -.023 & & & \\
\hline & \multicolumn{12}{|c|}{ Missing $X \sim Y(25 \%)$} \\
\hline$\beta_{W, Y X}(.350)$ & -.003 & -.005 & -.018 & .945 & .897 & .912 & .002 & .002 & -.020 & .952 & .902 & .918 \\
\hline$\beta_{B, Y X}(.600)$ & -.000 & -.001 & .001 & .938 & .942 & .939 & .010 & .010 & .022 & .946 & .953 & .949 \\
\hline$\tau_{0}^{2} \quad(.192)$ & .025 & .006 & .016 & & & & .024 & .005 & .011 & & & \\
\hline$(.200)$ & -.023 & -.089 & -.052 & & & & -.022 & -.106 & -.062 & & & \\
\hline
\end{tabular}

Note. Biases larger than 5\% and coverage values lower than $90 \%$ are written in bold. True values are given in parentheses. $\beta_{W, Y X}=$ within-group regression coefficient; $\beta_{B, Y X}=$ between-group regression coefficient; $\tau_{0}^{2}=$ intercept variance; $\tau_{1}^{2}=$ slope variance; $\mathrm{CD}=$ complete data; $\mathrm{PAN}=$ two-level joint model; FCS $=$ two-level conditional model.

$95 \%$. This could be explained by the fact that the slope variance is ignored in the imputation model of the PAN approach and that this in turn results in standard errors that are too small because this extra uncertainty is not taken into account when the imputations are generated (see Carpenter \& Kenward, 2013).

In the more challenging condition where the predictor variable is incomplete $(X \sim Y)$, the results for the PAN approach were almost identical, and the PAN approach continued to provide approximately unbiased estimates for the regression coefficients. However, as we see, the FCS approach underestimated the slope variance. The estimated relative bias $\left(-29 \%\right.$ for $\left.\tau_{1}^{2}=.10\right)$ matches the relative bias that is reported by Enders et al. (in press; see Table 2) under similar conditions. Furthermore, the FCS approach led to a small positive bias for the between-group regression coefficient (under MAR) and a small negative bias 


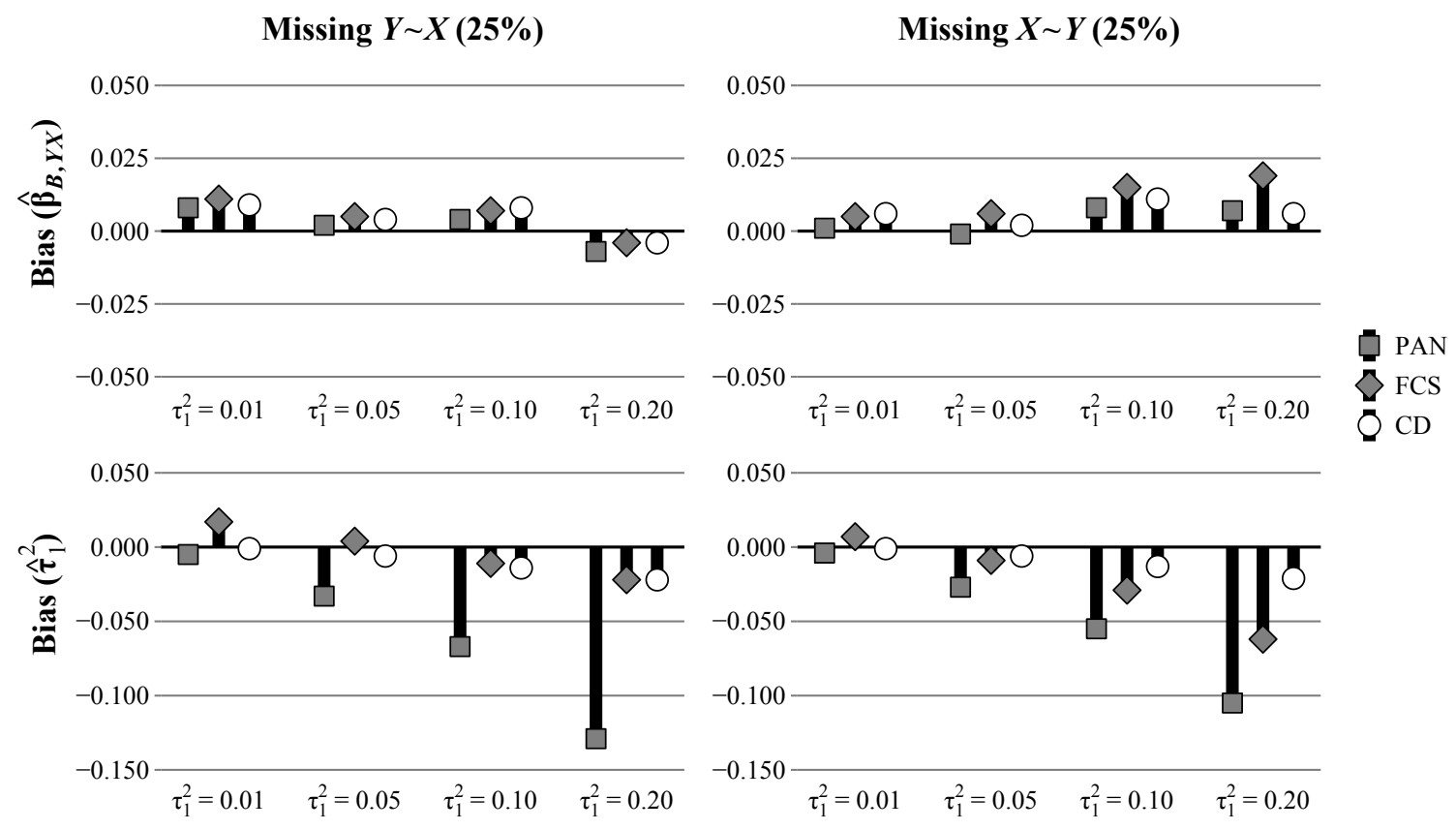

Figure 1. Bias for the between-group regression coefficient and the slope variance for different values of the (true) slope variance $\left(\tau_{1}^{2}\right)$. PAN $=$ two-level joint model; FCS = two-level conditional model; $\mathrm{CD}=$ complete data.

for the within-group regression coefficient (under both MAR and MCAR). As shown in Figure 1, the magnitude of the biases depended on the size of the true slope variance.

For very small values of the slope variance, neither the PAN nor the FCS approach produced biased estimates. However, as the true slope variance increased $\left(\tau_{1}^{2}=.05\right.$ or larger), both the PAN and the FCS approach provided negatively biased estimates of the slope variance. We also see that the bias for the estimator of the between-group coefficient in the FCS approach was relatively low but increased in size as the true slope grew larger.

In conclusion, the additional simulation suggested that the PAN approach also seems to provide approximately unbiased estimators of the within-group and between-group regression coefficients in models with random slopes. As expected, the estimates of the slope variance provided by the PAN approach were negatively biased. When the predictor variable is incomplete (i.e., the variable with a random slope), also the FCS approach 
produced biased estimates of the slope variance. These results suggest that estimates of slope variation in multilevel models with random slopes and incomplete predictor variables should be interpreted with caution (see also Grund, Lüdtke, \& Robitzsch, in press).

\section{Supplement B: Equivalence of Multilevel Imputation Models with Latent and Manifest Group Means}

There are two main approaches to estimating multilevel models with contextual effects. In the first approach, the group mean of the level-1 predictor variable is treated as an observed or manifest variable (see Raudenbush \& Bryk, 2002). This approach has traditionally been used in multilevel modeling and can result in biased estimates of group-level effects (especially when the ICC of the predictor $X$ is low and/or the group sizes are small). In the second approach, the group mean of the level-1 predictor is treated as latent (multilevel latent covariate model; see Lüdtke et al., 2008), and the group-level effect (i.e., the betweengroup effect) is controlled for error that is due to measuring a latent mean through the mean of a sample of finite size. This approach is currently the default in the software Mplus, and it is the one we use in our study.

The simulation results of our study show that if a researcher is interested in using the second approach (with latent means), the PAN approach is appropriate under most of the included conditions - problems were mainly observed with small groups and low ICC. This finding is consistent with the imputation model of the PAN approach ("empty model"; see Equation 24), which preserves the relations at the group level by treating the group means (or between parts of the level-1 variables) as latent variables. It is an important question, whether the PAN approach can also be used when the model of interest is a multilevel model with manifest group means. To answer this question, we conducted an additional simulation in which the multilevel random-intercept model with manifest means was the analysis model (and also the data-generating model). More specifically, for the model with manifest means

we used the observed group mean of the predictor $X$ by calculating $\bar{X}_{\bullet j}=\sum_{i=1}^{n_{j}} X_{i j}$ and treat 
it as a manifest variable (see Raudenbush \& Bryk, 2002):

$$
Y_{i j}=\beta_{0}+\beta_{W, Y X}^{\operatorname{man}}\left(X_{i j}-\bar{X}_{\bullet j}\right)+\beta_{B, Y X}^{\operatorname{man}} \bar{X}_{\bullet j}+\varepsilon_{i j}
$$

where $\beta_{0}$ is the regression intercept, $\beta_{W, Y X}^{\operatorname{man}}$ is the within-group regression coefficient describing the relationship between $Y$ and $X$ within groups, and $\beta_{B, Y X}^{\operatorname{man}}$ is the between-group regression coefficient that indicates the relationship between group means. The crucial question is whether the PAN approach is also appropriate if the multilevel model with manifest group means is the model of interest.

First, we assumed that the multilevel random-intercept model with manifest group means holds in the population (see Study 3 in Lüdtke et al., 2008, for a description of the data-generating procedure). We used selected conditions of the simulation in the main article and varied the group size ( $n=5$ and 15), the ICC of $X$ and $Y(.10$ and .30), and the missing mechanism (MCAR and MAR). The other conditions were held constant: number of groups $(K=150)$, within-group correlation $\left(\rho_{W}=.35\right)$, between-group correlation $\left(\rho_{B}\right.$ $=.60)$, and missing data rate $(25 \%)$. For each data set, missing data were imputed using the PAN approach. After imputing the missing values on $Y$, data sets were analyzed with a multilevel random-intercept model with manifest group means for the level-1 predictor using Mplus 7.3. We focused on the scenario when missing values occur on the predictor variable.

Table 2 shows that the PAN approach produces approximately unbiased estimates of the between-group regression coefficient (i.e., constellation with an incomplete predictor variable $Y$ and a fully observed dependent variable $X$ ) with coverage rates near the nominal value in most conditions. In conditions with a small intraclass correlation and a small group size, the estimator provided by the PAN approach was slightly negatively biased $(-14.5 \%$ in relative terms). However, the magnitude of this bias was comparable to the bias of the PAN approach when the analysis model (and data-generating model) was the multilevel 
Table 2

Bias and Coverage of the $95 \%$ Confidence Interval for the Estimators of the Between-Group Regression Coefficient in a Multilevel Model with Manifest Group Means $\left(\beta_{B, Y X}^{\operatorname{man}}\right)$ for a Large Number of Groups $(K=150)$

\begin{tabular}{|c|c|c|c|c|c|c|c|c|c|}
\hline \multirow[b]{3}{*}{$\rho_{I}$} & \multirow[b]{3}{*}{$n$} & \multicolumn{4}{|c|}{$\operatorname{MCAR}(\lambda=0)$} & \multicolumn{4}{|c|}{$\operatorname{MAR}(\lambda=0.8)$} \\
\hline & & \multicolumn{2}{|c|}{ Bias } & \multicolumn{2}{|c|}{ Coverage $(95 \%)$} & \multicolumn{2}{|c|}{ Bias } & \multicolumn{2}{|c|}{ Coverage (95\%) } \\
\hline & & PAN & $\mathrm{CD}$ & PAN & $\mathrm{CD}$ & PAN & $\mathrm{CD}$ & PAN & $\mathrm{CD}$ \\
\hline 0.1 & 5 & -.087 & .003 & 94.3 & 93.9 & -.086 & .003 & 95.2 & 93.9 \\
\hline 0.1 & 15 & -.024 & -.002 & 95.3 & 92.5 & -.030 & -.002 & 95.5 & 92.5 \\
\hline 0.3 & 5 & -.010 & .003 & 95.6 & 94.6 & -.013 & .003 & 96.2 & 94.6 \\
\hline 0.3 & 15 & .001 & -.001 & 94.6 & 93.9 & -.004 & -.001 & 94.5 & 93.3 \\
\hline
\end{tabular}

Note. Biases larger than $10 \%$ and coverage values lower than $90 \%$ are written in bold. True value $\beta_{B, Y X}=$ .600. $\rho_{I}=$ intraclass correlation of $X$ and $Y ; n=$ group size; $\mathrm{CD}=$ complete data; $\mathrm{PAN}=$ two-level joint model.

random-intercept model with latent group means ( $-15.2 \%$; see Table 2 in the main article).

In the following, we provide a formal argument explaining why the PAN approach is also appropriate for the imputation of missing data, when the multilevel model with manifest group means holds in the population. Vice versa, this argument also suggests that a multilevel model with manifest group means can be used when the population model is the one with latent group means, thus making both models suitable for MI in either case.

The main idea is that the covariance structure of the empty model of the PAN approach (see Equation 24 in the main article) can be equivalently represented by a multilevel model with manifest group means (see Carpenter \& Kenward, 2013, p. 221). Consider a two-level structure in which two individual-level variables $X_{i j}$ and $Y_{i j}$ are decomposed into latent within- and between-group components (see Equation 3 in the main article). Then, the covariance matrices of $X$ and $Y$ within and between groups can be written as follows:

$$
\boldsymbol{\Sigma}_{W}=\left(\begin{array}{cc}
\sigma_{X}^{2} & \rho_{W} \sigma_{X} \sigma_{Y} \\
\rho_{W} \sigma_{X} \sigma_{Y} & \sigma_{Y}^{2}
\end{array}\right) \quad \text { and } \quad \boldsymbol{\Sigma}_{B}=\left(\begin{array}{cc}
\tau_{X}^{2} & \rho_{B} \tau_{X} \tau_{Y} \\
\rho_{B} \tau_{X} \tau_{Y} & \tau_{Y}^{2}
\end{array}\right)
$$


where $\Sigma_{W}$ is a within-group covariance matrix and $\boldsymbol{\Sigma}_{B}$ a between-group covariance matrix. Let us further assume that the within and between components are normally distributed and the group sizes are balanced (with size $n$ ). It has been shown in the literature that the bivariate multilevel random-intercept model can also be represented as a multivariate single level model with $2 \cdot n$ variables (Mehta \& Neale, 2005, pp. 265). Let $\mathbf{X}_{j}=\left(X_{1 j}, \ldots, X_{n j}\right)$ and $\mathbf{Y}_{j}=\left(Y_{1 j}, \ldots, Y_{n j}\right)$ denote the vectors for $X$ and $Y$ variables of the persons $i$ in a specific group $j$. Carpenter and Kenward (2013, p. 221; see also Mehta \& Neale, 2005) show that the vector $\left(\mathbf{X}_{j}, \mathbf{Y}_{j}\right)$ for a group $j$ is multivariate normally distributed with a mean vector including $2 \cdot n$ means of $X$ and $Y$, and a covariance matrix

$$
\boldsymbol{\Sigma}=\left(\begin{array}{cc}
\tau_{X}^{2} \mathbf{J}+\sigma_{X}^{2} \mathbf{I} & \rho_{B} \tau_{X} \tau_{Y} \mathbf{J}+\rho_{W} \sigma_{X} \sigma_{Y} \mathbf{I} \\
\rho_{B} \tau_{X} \tau_{Y} \mathbf{J}+\rho_{W} \sigma_{X} \sigma_{Y} \mathbf{I} & \tau_{Y}^{2} \mathbf{J}+\sigma_{Y}^{2} \mathbf{I}
\end{array}\right)
$$

where $\mathbf{J}$ is $n \times n$ matrix in which all entries are equal to one, and $\mathbf{I}$ is the $n \times n$ identity matrix which has ones in the diagonal and zeros as all other entries. Generating imputations from the bivariate multilevel normal model (i.e., the PAN approach) when only some of the $\mathbf{Y}_{j}$ values are missing basically means drawing imputations from the conditional distribution $P\left(\mathbf{Y}_{j} \mid \mathbf{X}_{j}\right)$. Note that the joint distribution $P\left(\mathbf{X}_{j}, \mathbf{Y}_{j}\right)$ is also given by the product of the conditional distribution $P\left(\mathbf{Y}_{j} \mid \mathbf{X}_{j}\right)$ and the marginal distribution $P\left(\mathbf{X}_{j}\right)$, i.e. $P\left(\mathbf{X}_{j}, \mathbf{Y}_{j}\right)=P\left(\mathbf{X}_{j}\right) \cdot P\left(\mathbf{Y}_{j} \mid \mathbf{X}_{j}\right)$. As the joint distribution $P\left(\mathbf{X}_{j}, \mathbf{Y}_{j}\right)$ is multivariate normal, the conditional distribution $P\left(\mathbf{Y}_{j} \mid \mathbf{X}_{j}\right)$ is also multivariate normal. Using a formula for the inversion of a structured covariance matrix (Magnus \& Neudecker, 1988), it can be shown that the conditional mean of the distribution $P\left(\mathbf{Y}_{j} \mid \mathbf{X}_{j}\right)$ is a function of the vector $\mathbf{X}_{j}$ and the manifest group mean (Carpenter \& Kenward, 2013, p. 221). The conditional mean of each component $E\left(Y_{i j} \mid \mathbf{X}_{j}\right)$ is then given by

$$
E\left(Y_{i j} \mid \mathbf{X}_{j}\right)=\beta_{0}+\beta_{W, Y X}^{m a n}\left(X_{i j}-\bar{X}_{\bullet j}\right)+\beta_{B, Y X}^{m a n} \bar{X}_{\bullet j}
$$


where the coefficients $\beta_{W, Y X}^{\operatorname{man}}=\rho_{W} \sigma_{Y} / \sigma_{X}$ and $\beta_{B, Y X}^{\operatorname{man}}=\left(\rho_{B} \tau_{X} \tau_{Y}+\rho_{W} \sigma_{X} \sigma_{Y} / n\right) /\left(\tau_{X}^{2}+\sigma_{X}^{2} / n\right)$ can be directly calculated from the entries of the covariance matrices $\Sigma_{W}$ and $\Sigma_{B}$ (Carpenter \& Kenward, 2013, p. 221). Furthermore, the conditional covariance of the distribution $P\left(\mathbf{Y}_{j} \mid \mathbf{X}_{j}\right)$ can be written as $\tau_{e}^{2} \mathbf{J}+\sigma_{e}^{2} \mathbf{I}$ with variance components $\tau_{e}^{2}=\tau_{Y}^{2}-\left(\rho_{B} \tau_{X} \tau_{Y}+\right.$ $\left.\rho_{W} \sigma_{X} \sigma_{Y} / n\right)^{2} /\left(\tau_{X}^{2}+\sigma_{X}^{2} / n\right)$ and $\sigma_{e}^{2}=\sigma_{Y}^{2}\left(1-\rho_{W}^{2}\right)$. These findings show that the conditional distribution $P\left(\mathbf{Y}_{j} \mid \mathbf{X}_{j}\right)$ is consistent with a multilevel random-intercept model with manifest group means with a level-2 variance $\tau_{e}^{2}$ and level-1 variance $\sigma_{e}^{2}$. Therefore, drawing imputations from the model with manifest group means is an appropriate imputation strategy when the multilevel model with latent group means holds in the population.

A similar argument can be used in the opposite case, if the PAN approach is used as an imputation model and the analysis model is a multilevel model with manifest group means. In this scenario, one has to show that the multivariate distribution $P\left(\mathbf{X}_{j}, \mathbf{Y}_{j}\right)$ of the PAN approach (Equation 4) follows, if the multilevel model with manifest group means holds in the population. Let us again assume that $X_{i j}$ is decomposed into normally distributed between-group and within-group components (with corresponding variances $\tau_{X}^{2}$ and $\sigma_{X}^{2}$, respectively), and that group sizes are balanced. If the multilevel model with manifest group means holds, the conditional mean of the conditional distribution $P\left(\mathbf{Y}_{j} \mid \mathbf{X}_{j}\right)$ has the form given in Equation 5, and the conditional covariance matrix is given by $\tau_{e}^{2} \mathbf{J}+\sigma_{e}^{2} \mathbf{I}$. Using the relation $P\left(\mathbf{X}_{j}, \mathbf{Y}_{j}\right)=P\left(\mathbf{X}_{j}\right) \cdot P\left(\mathbf{Y}_{j} \mid \mathbf{X}_{j}\right)$, the joint distribution $P\left(\mathbf{X}_{j}, \mathbf{Y}_{j}\right)$ is then multivariate normal with covariance matrix

$$
\boldsymbol{\Sigma}=\left(\begin{array}{cc}
\tau_{X}^{2} \mathbf{J}+\sigma_{X}^{2} \mathbf{I} & \psi_{Y X} \mathbf{J}+\sigma_{Y X} \mathbf{I} \\
\psi_{Y X} \mathbf{J}+\sigma_{Y X} \mathbf{I} & \tau_{Y}^{2} \mathbf{J}+\sigma_{Y}^{2} \mathbf{I}
\end{array}\right)
$$

where all parameters in this matrix can be calculated as functions from the marginal distribution $P\left(\mathbf{X}_{j}\right)$ and the parameters from the model with manifest group means $P\left(\mathbf{Y}_{j} \mid \mathbf{X}_{j}\right)$. More specifically, it holds that $\psi_{Y X}=-\beta_{W, Y X}^{\operatorname{man}} \cdot 1 / n \cdot \sigma_{X}^{2}+\beta_{B, Y X}^{\operatorname{man}}\left(\tau_{X}^{2}+\sigma_{X}^{2} / n\right), \sigma_{Y X}=\beta_{W, Y X}^{\operatorname{man}} \sigma_{X}^{2}$, $\tau_{Y}^{2}=-\left(\beta_{W, Y X}^{\operatorname{man}}\right)^{2} \cdot 1 / n \cdot \sigma_{X}^{2}+\left(\beta_{B, Y X}^{\operatorname{man}}\right)^{2}\left(\tau_{X}^{2}+\sigma_{X}^{2} / n\right)+\tau_{e}^{2}$, and $\sigma_{Y}^{2}=\left(\beta_{W, Y X}^{\operatorname{man}}\right)^{2} \sigma_{X}^{2}+\sigma_{e}^{2}$. Hence, 
the covariance structure implied by the model with manifest group means is identical to the covariance structure of the empty model of the PAN approach. This result shows that the PAN approach can be used as an imputation model, even when the multilevel model with manifest group means is the model of interest.

Based on the finding of the equivalence of the imputation models with latent group means and manifest group means (for normally distributed multilevel data with balanced group sizes), we expect that imputations generated by a joint imputation approach (i.e., the PAN approach) and a fully conditional approach (using multilevel models with manifest group means as conditional distributions) perform similarly and will imply the same covariance structure among the variables (see Enders et al., in press). A similar argument was also made by Mistler $\underline{(2015)}$.

\section{Supplement C: R Code for the Data Analysis Example}

This section contains the $\mathrm{R}$ code used for the analysis example in which the different missing data methods have been applied to a large empirical dataset from educational research (students nested within classes). The example dataset included the class indicator variable, and five partially observed variables that were measured at the student level.

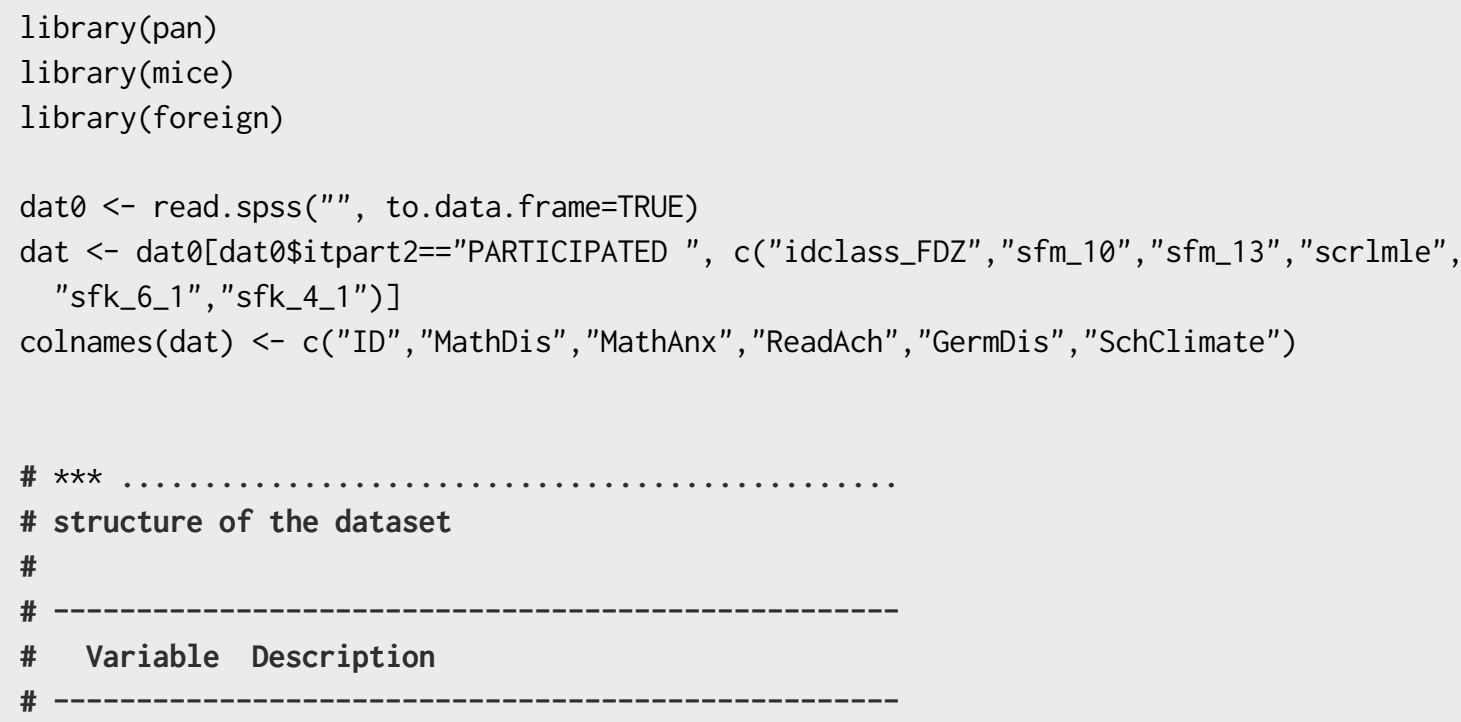




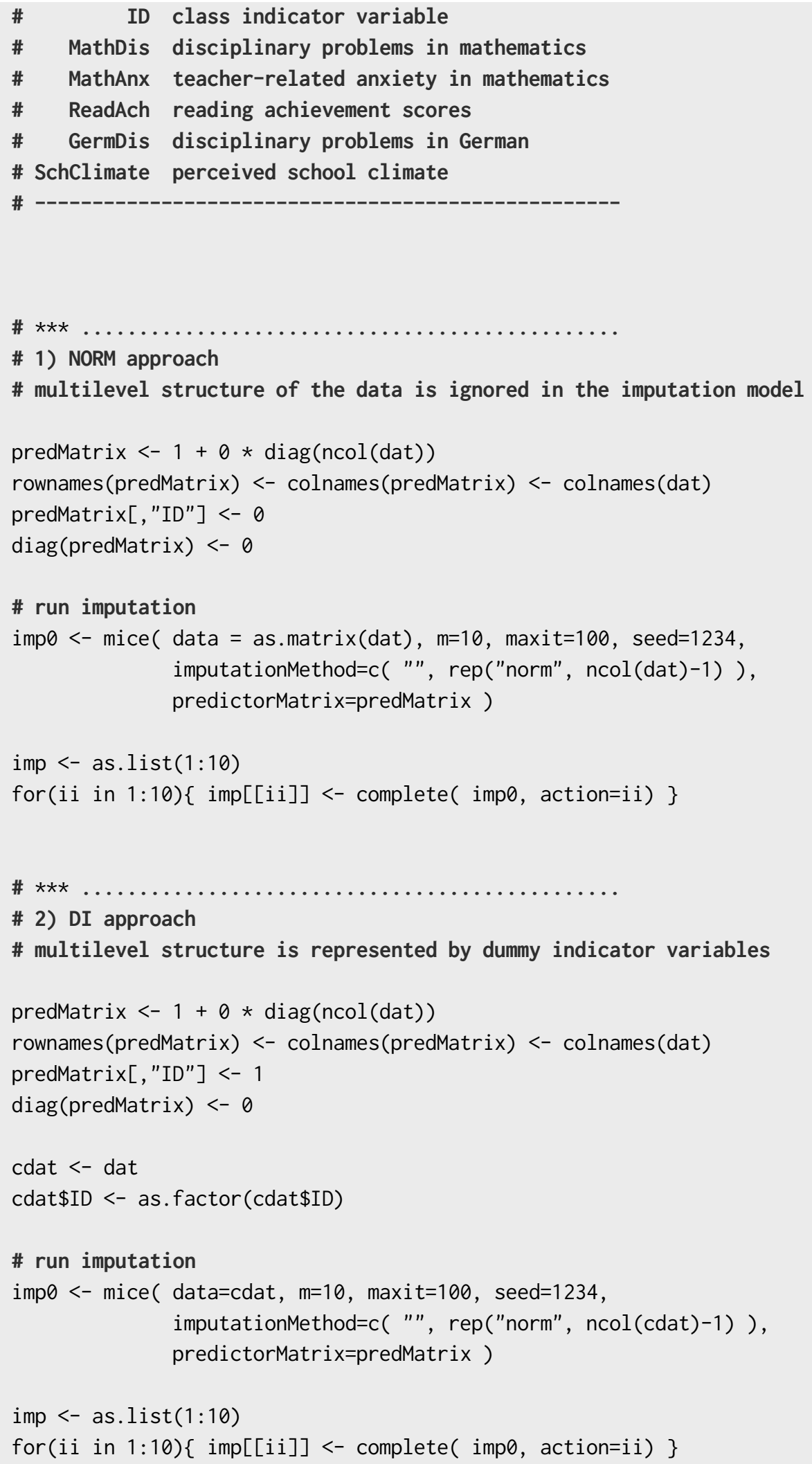




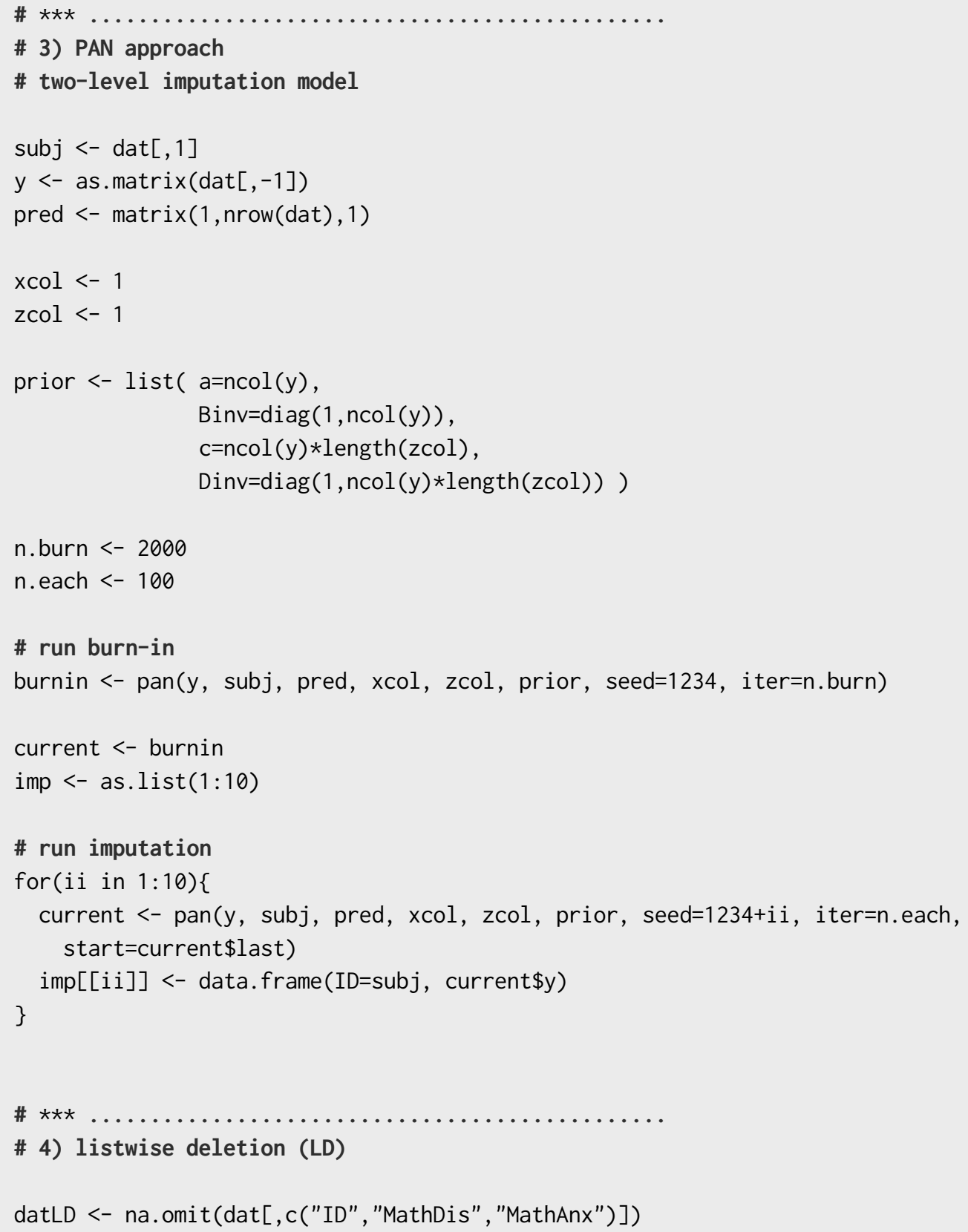




\section{Supplement D: Additional Tables}

The following pages contain the complete simulation results for the intraclass correlation of $Y$, and the between-group and within-group regression coefficients in the multilevel regression models of $X$ on $Y$ and $Y$ on $X$, respectively. We included the results for bias, RMSE, and (where possible) the coverage rates of the $95 \% \mathrm{CI}$. The results for the intraclass correlation of $Y$ are presented in Table A1 to A16. The results for the between- and withingroup regression coefficients with $X$ regressed on $Y$ are presented in Table B1 to B24 and Table C1 to C24, respectively. Table D1 to D24 and Table E1 to E24 contain the results for the between- and within-group regression coefficients for $Y$ regressed on $X$, respectively. 


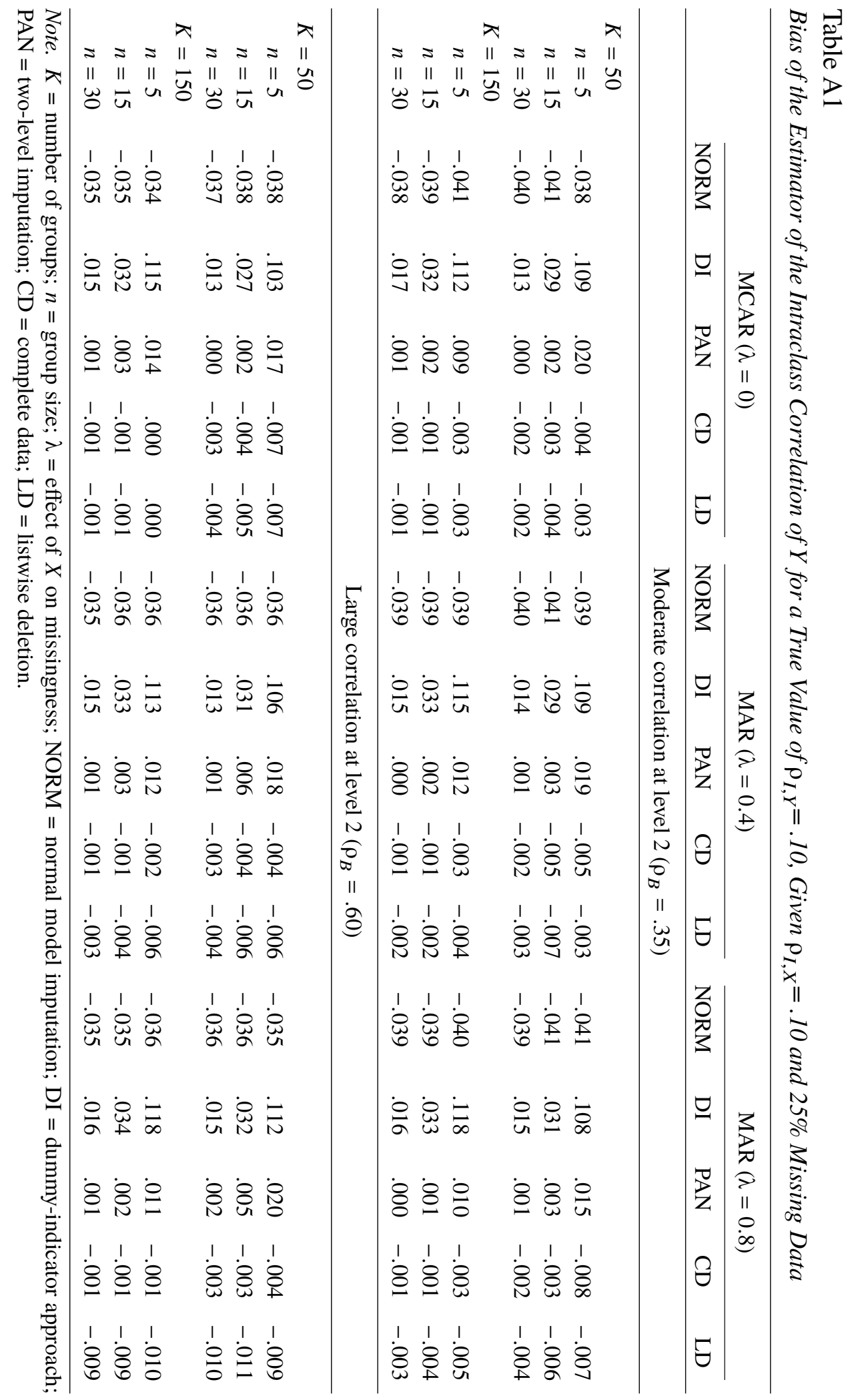




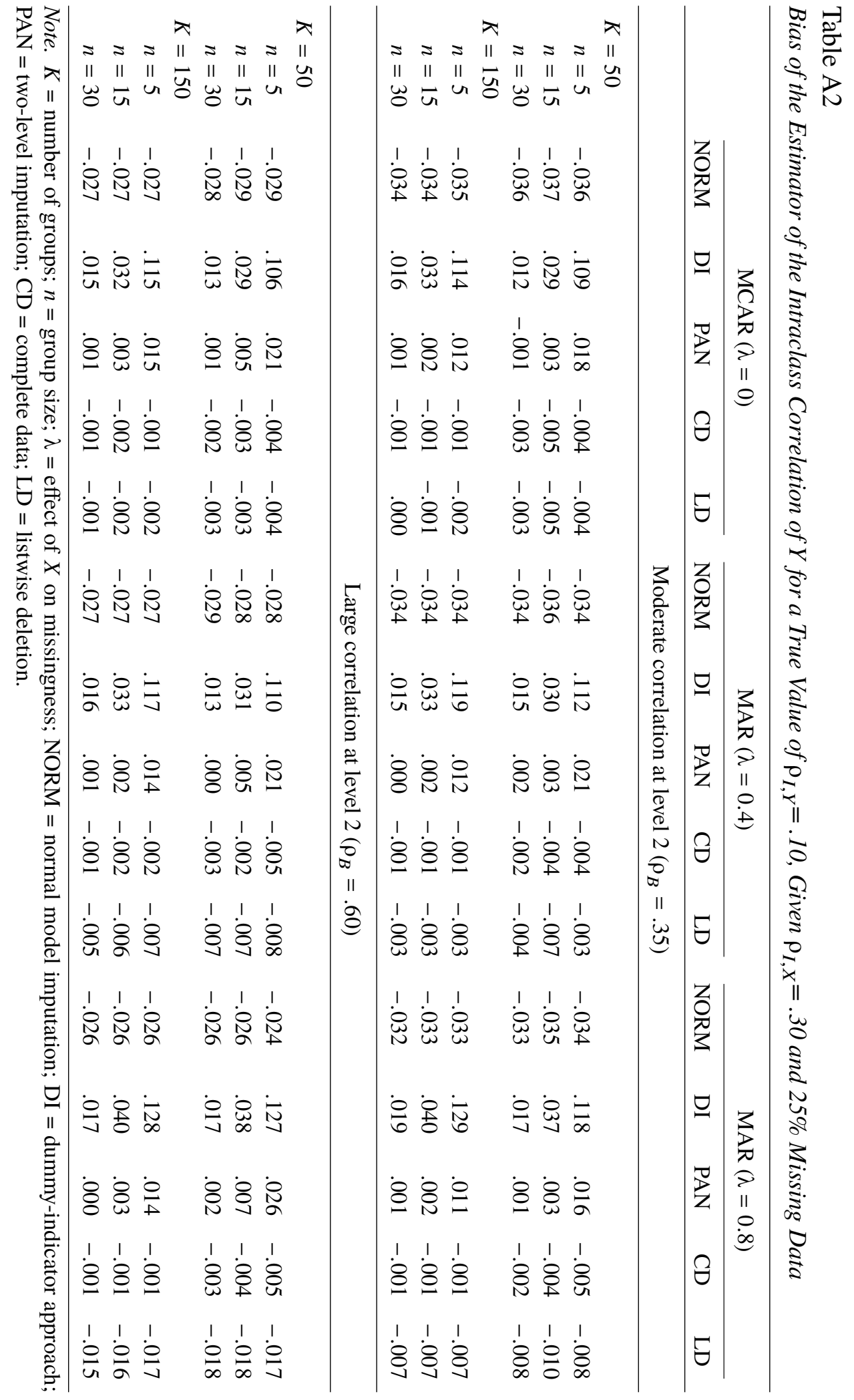




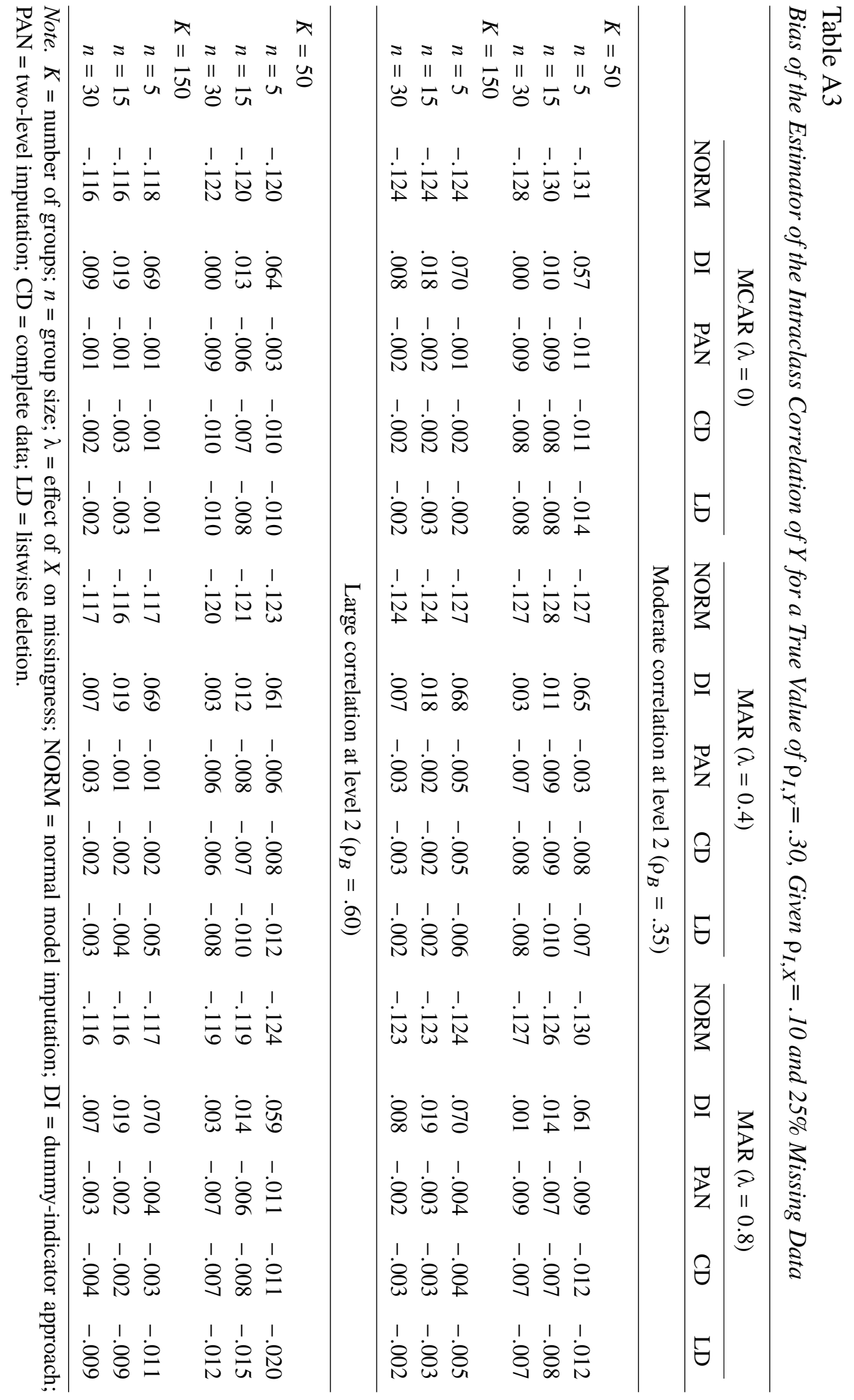




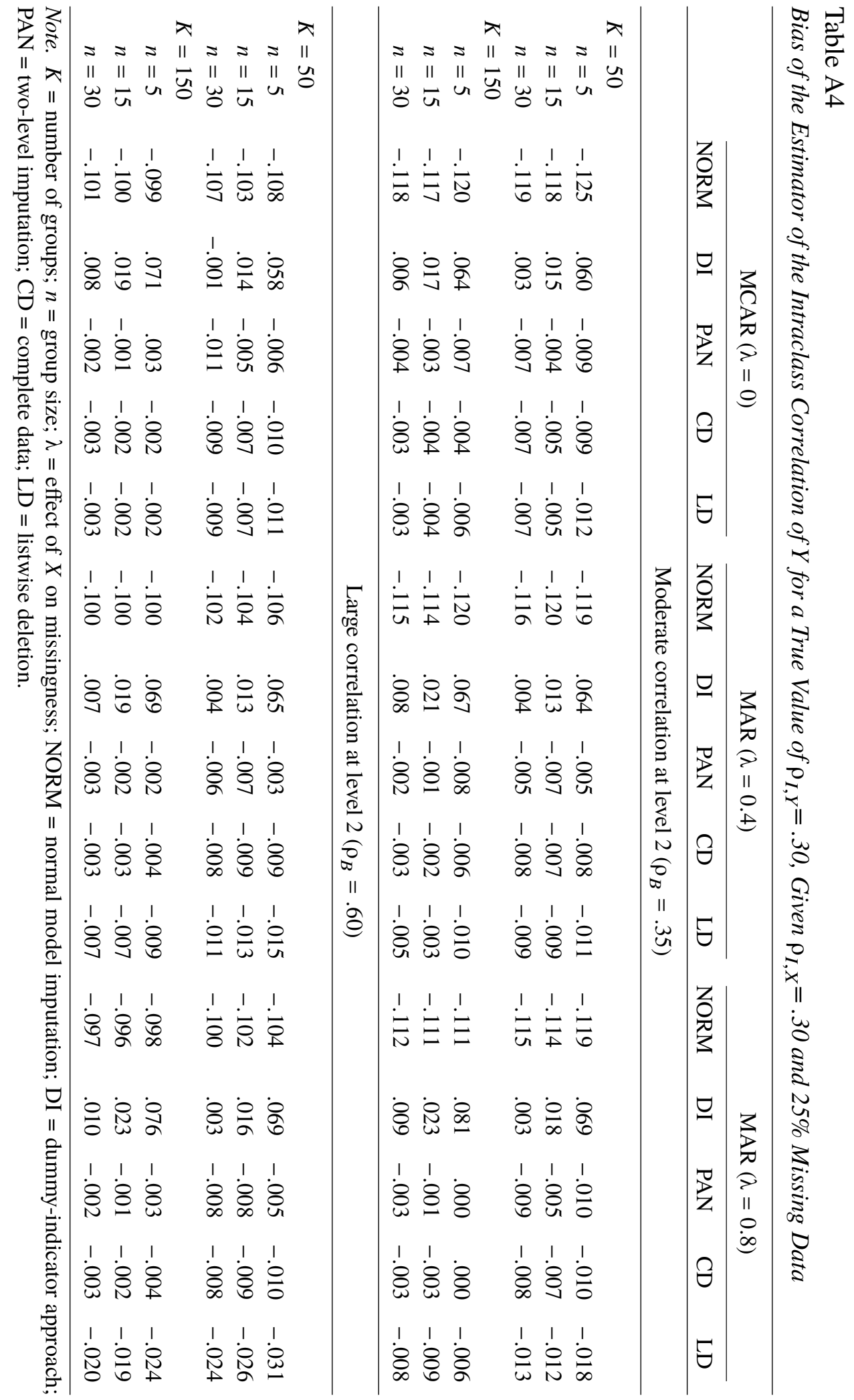




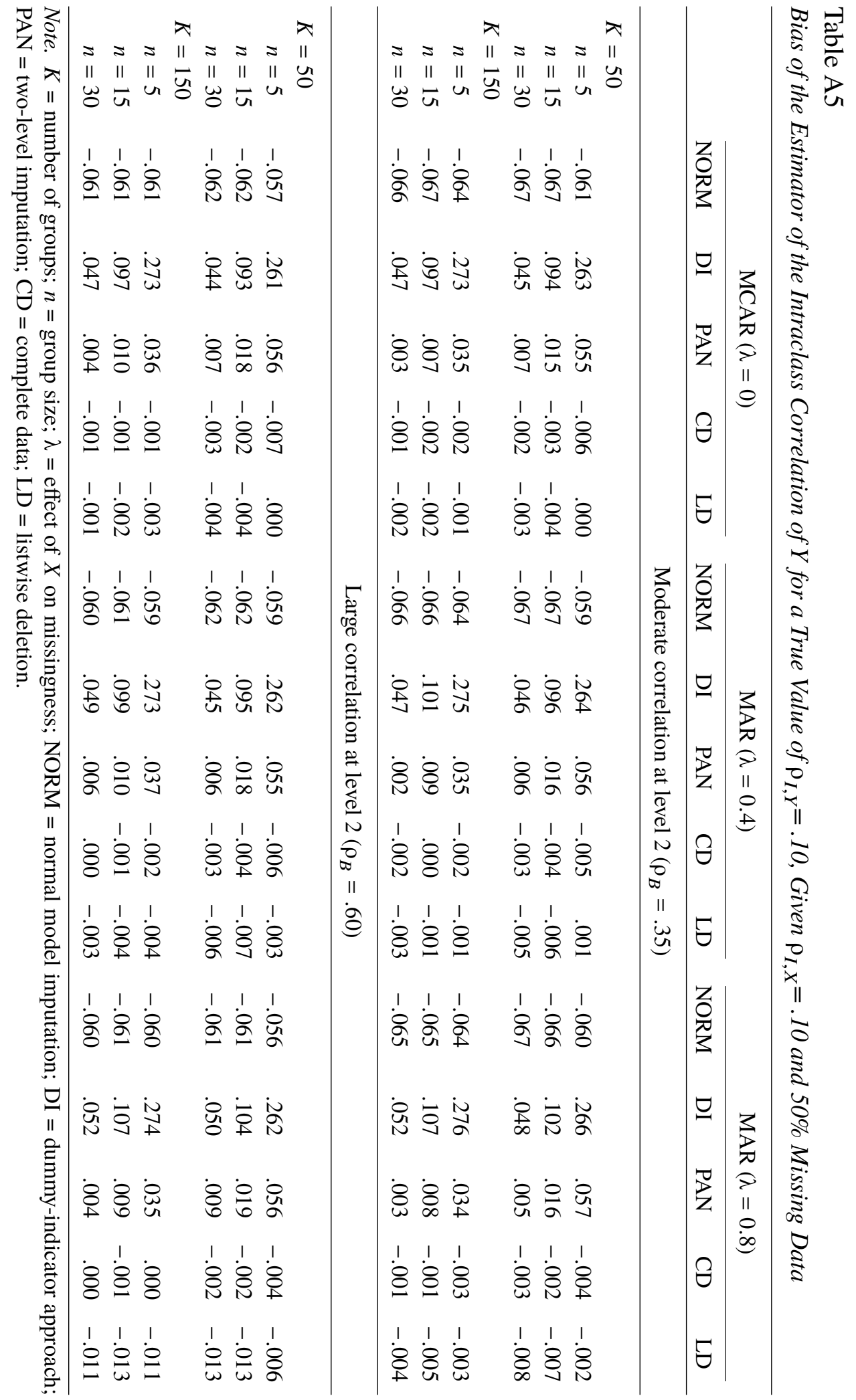




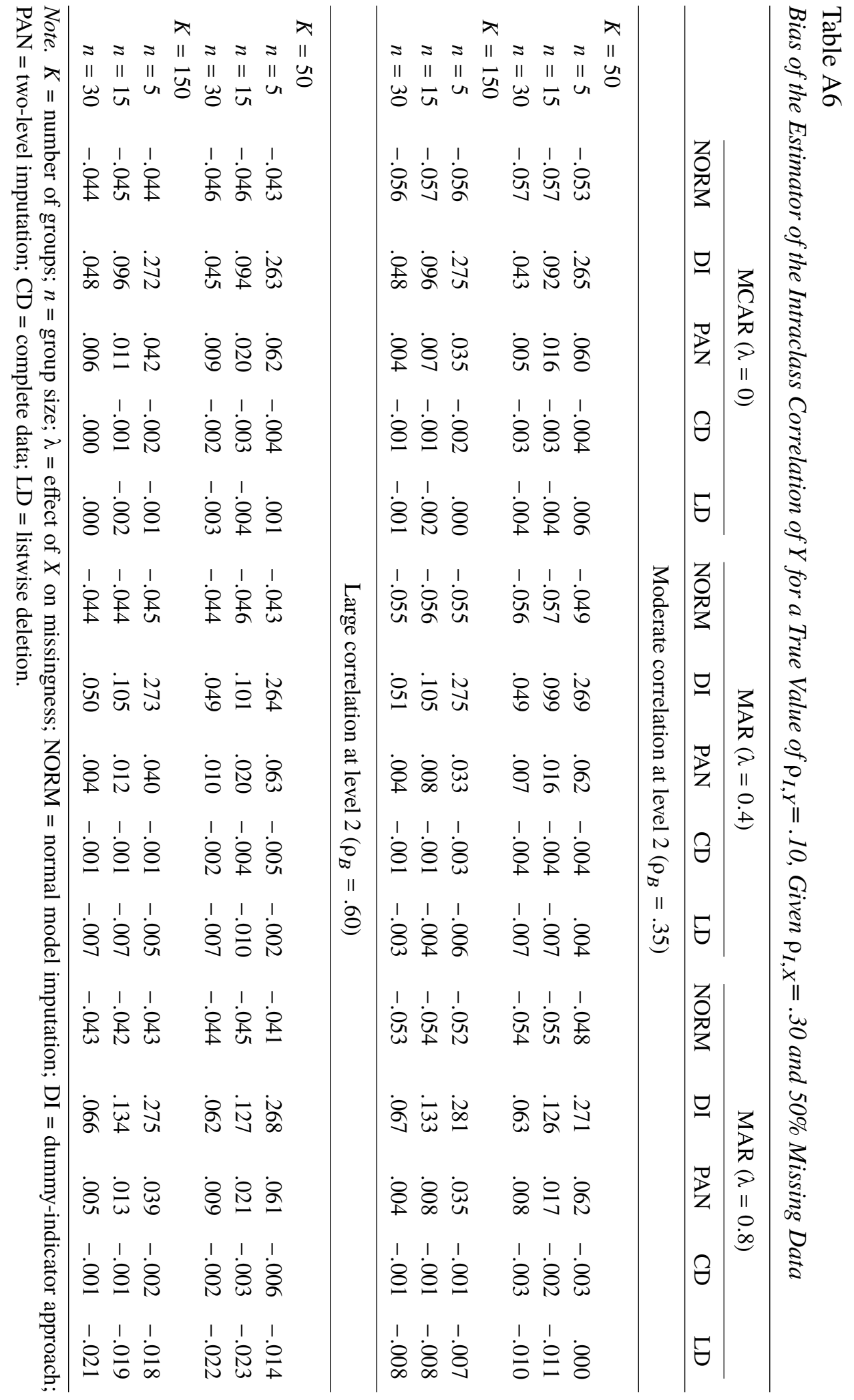




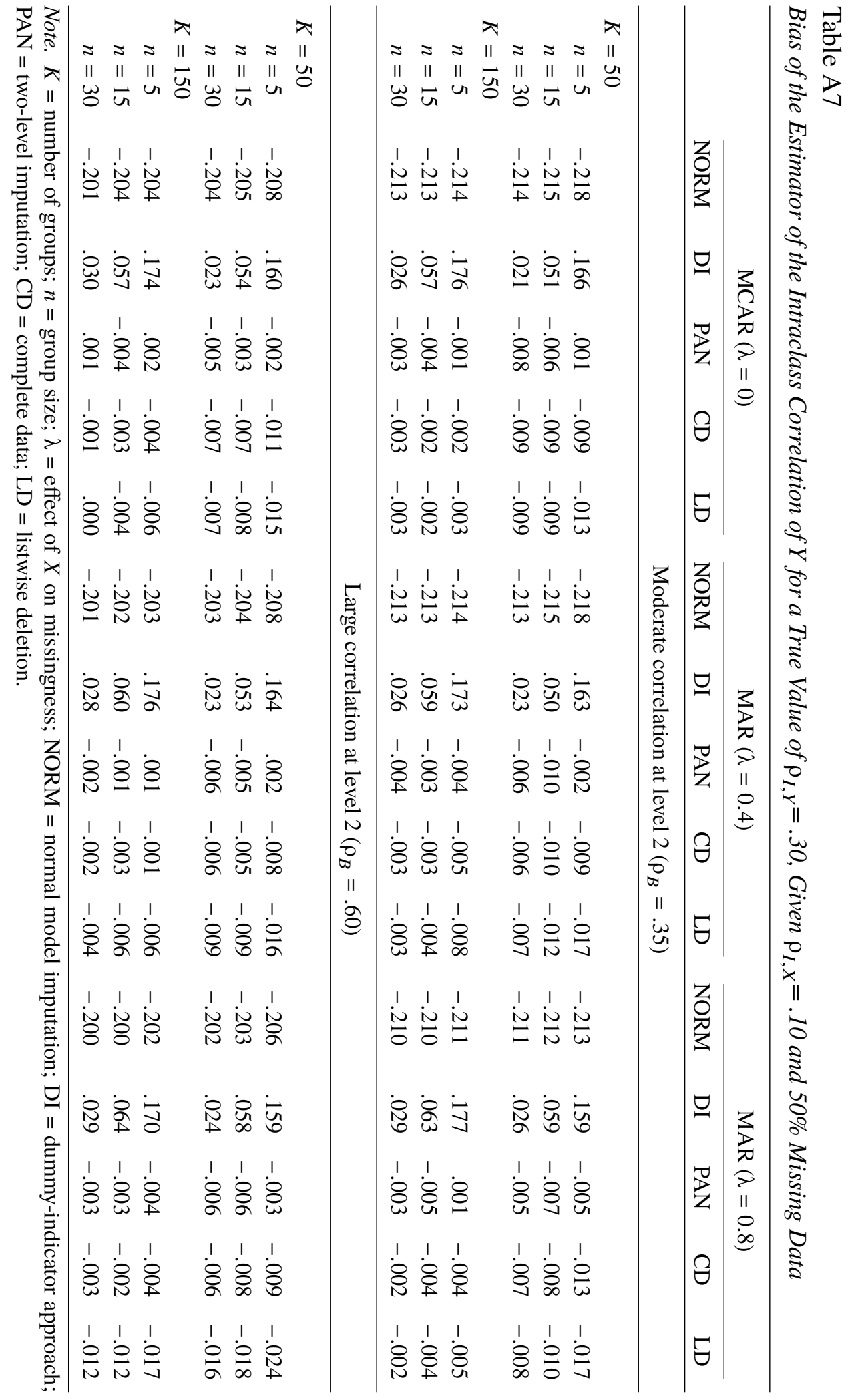




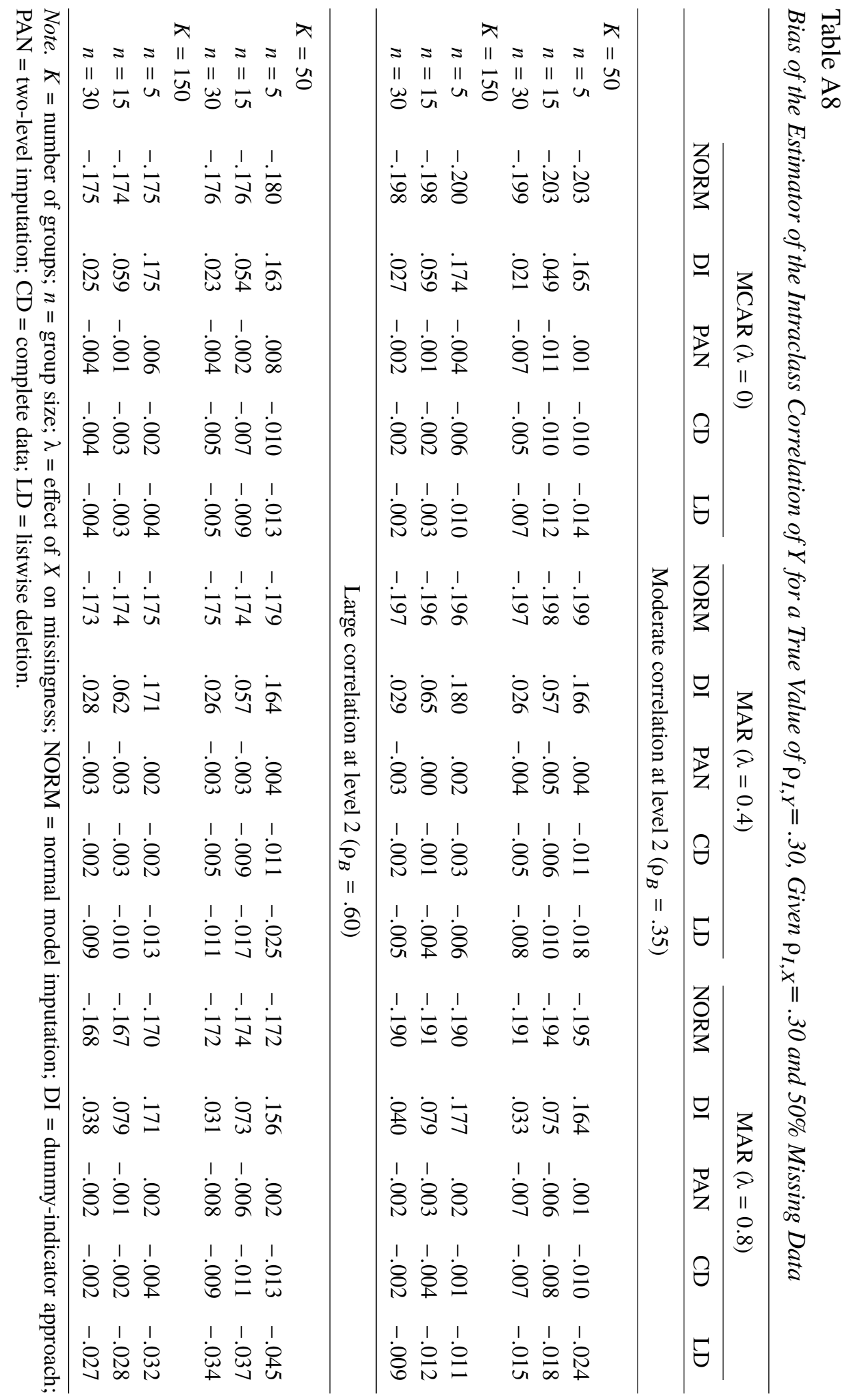




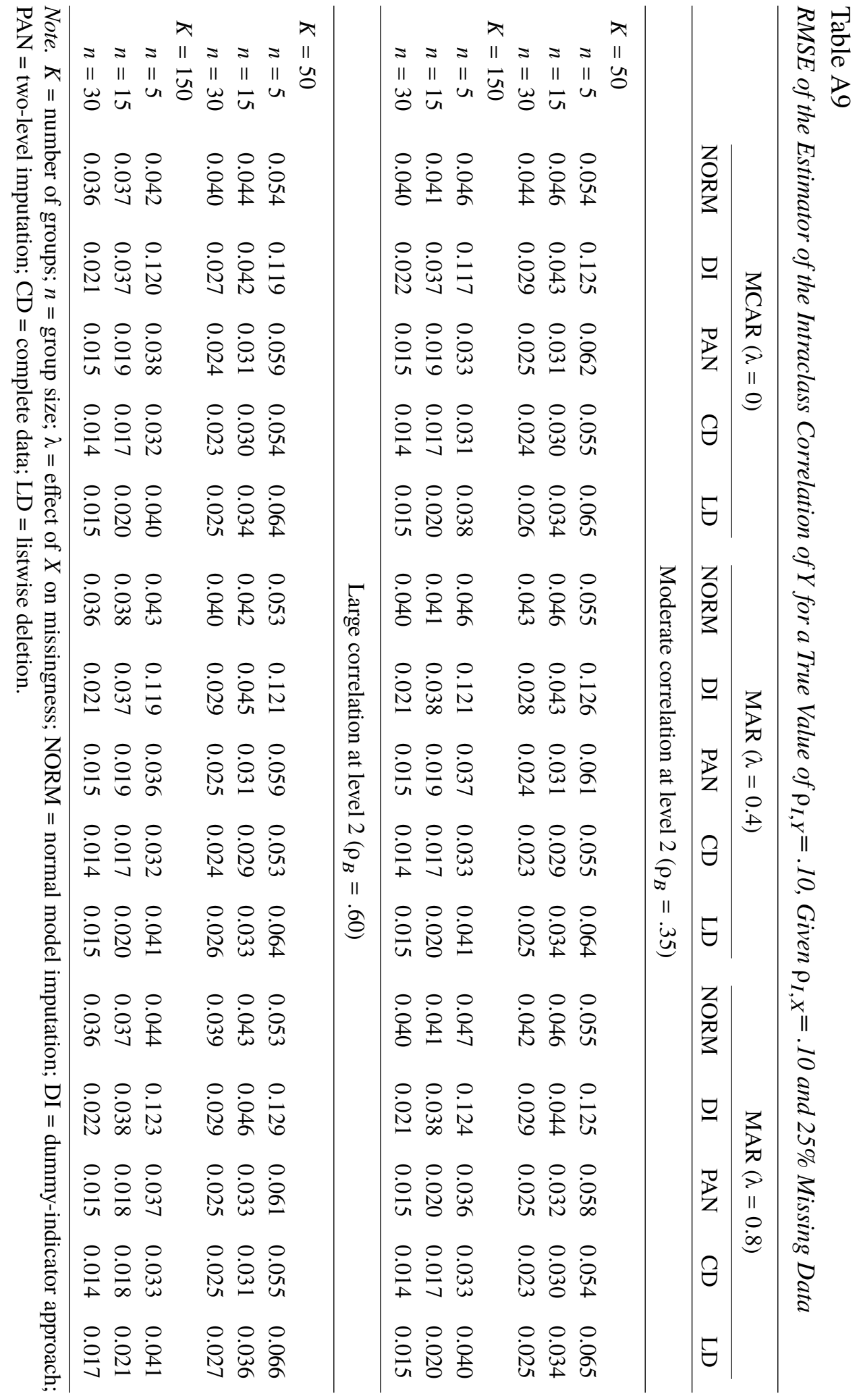




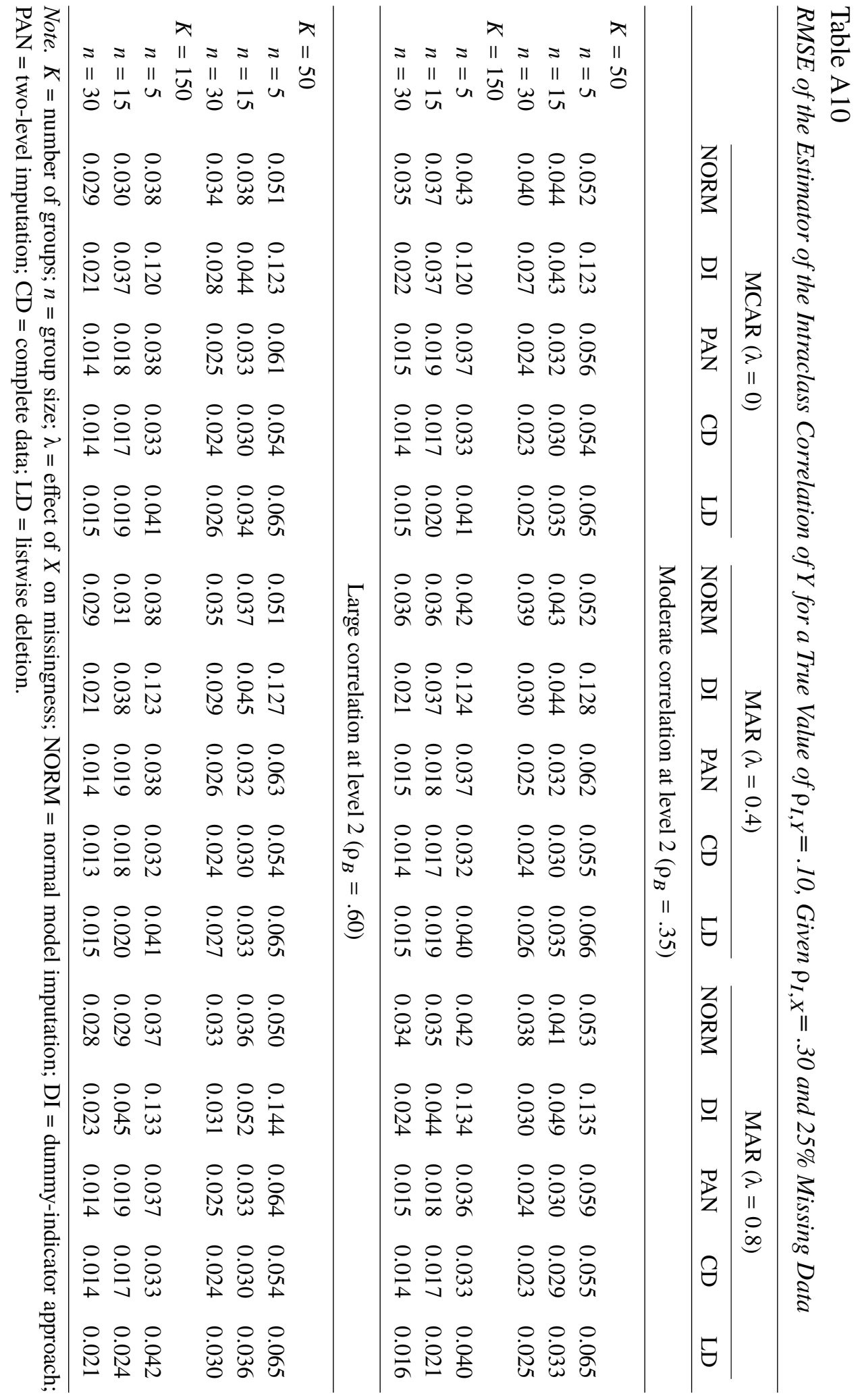




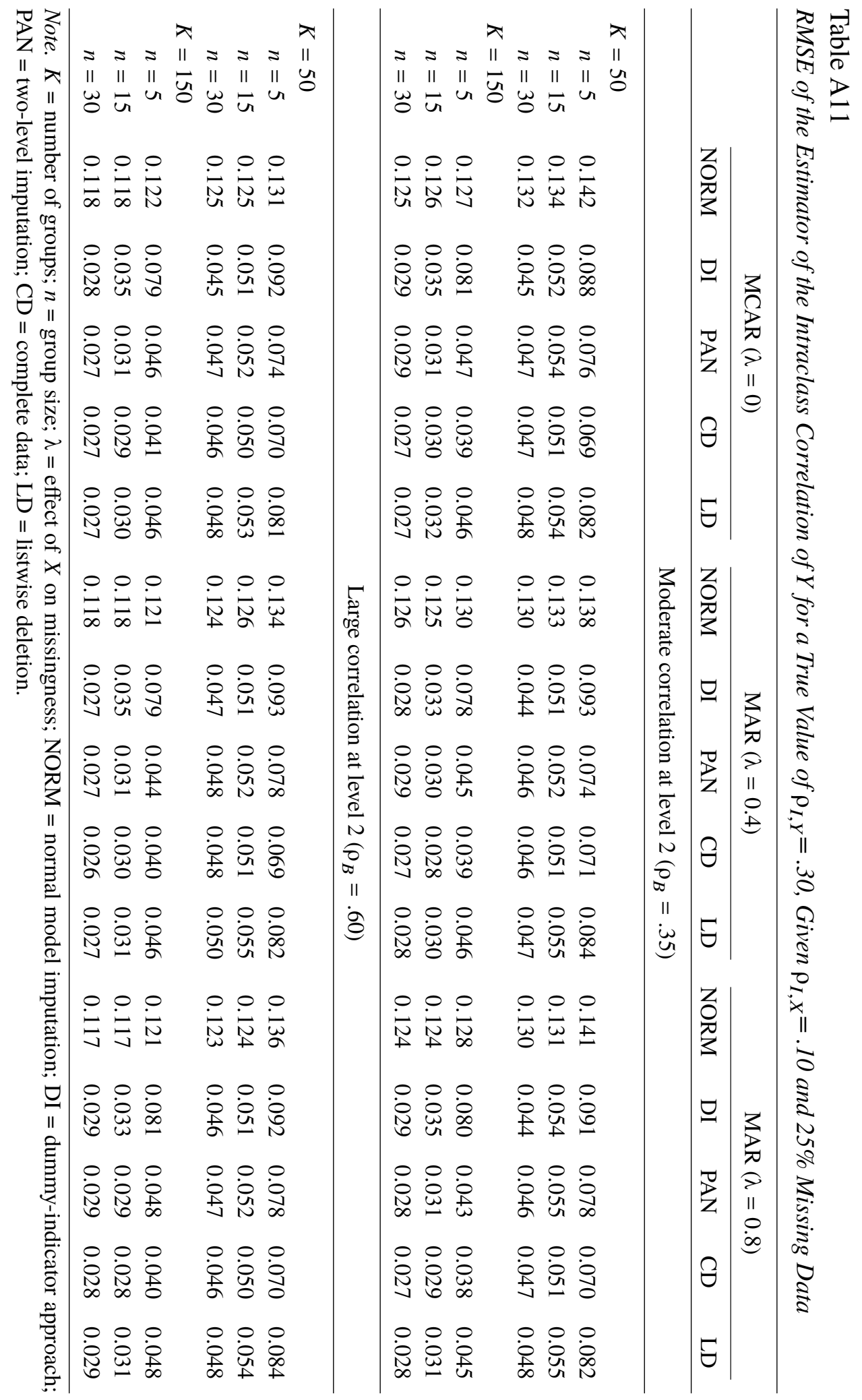




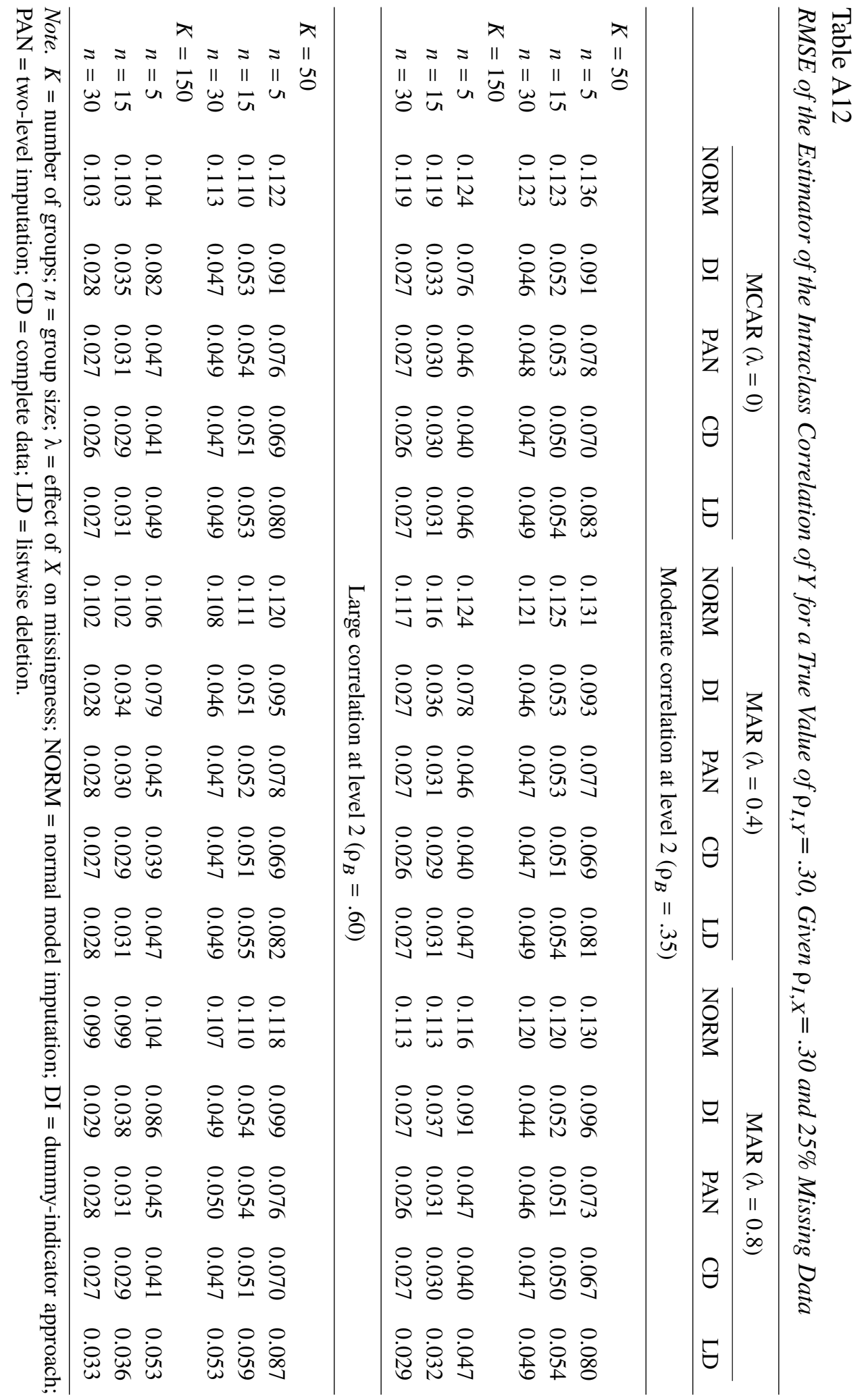




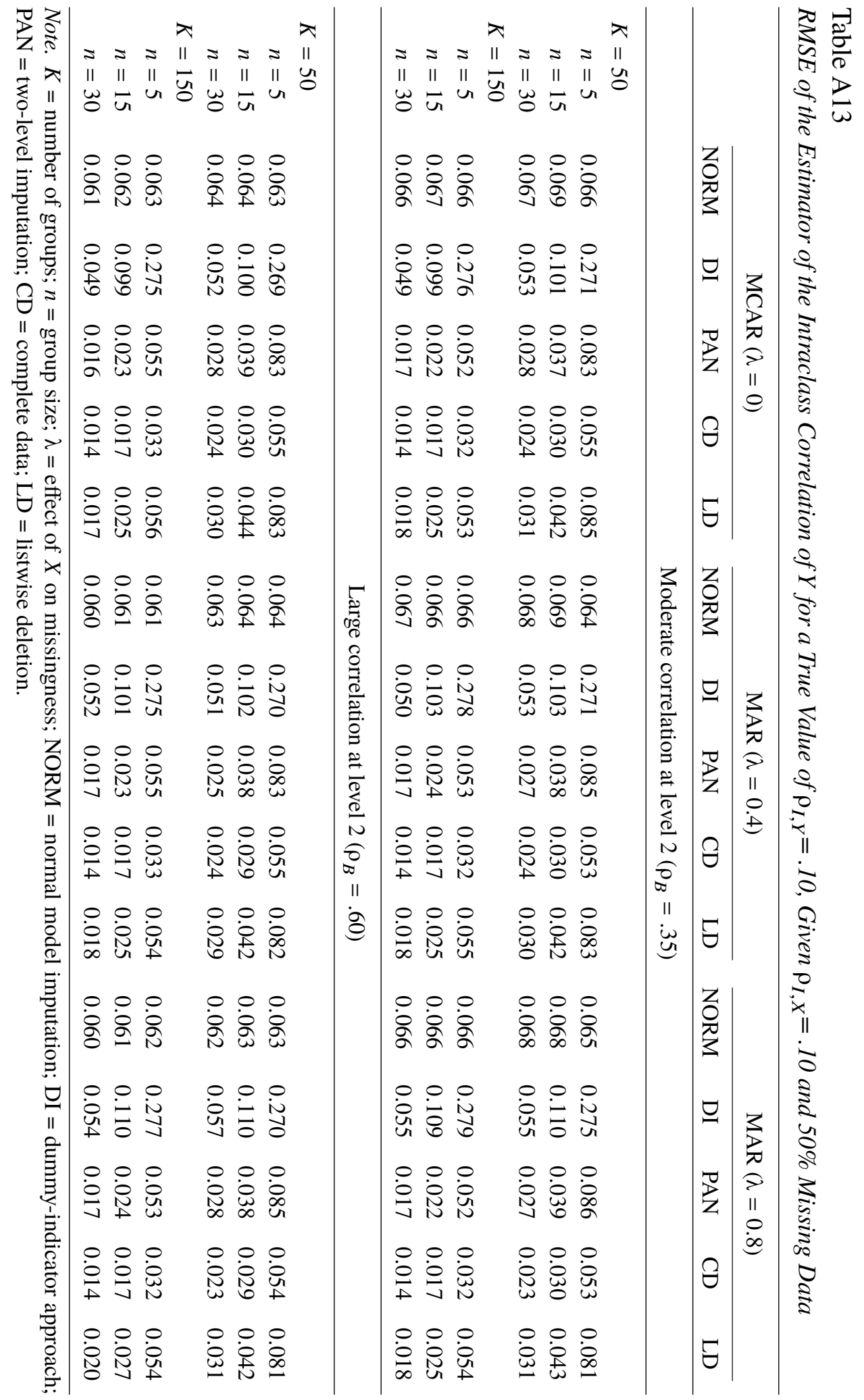




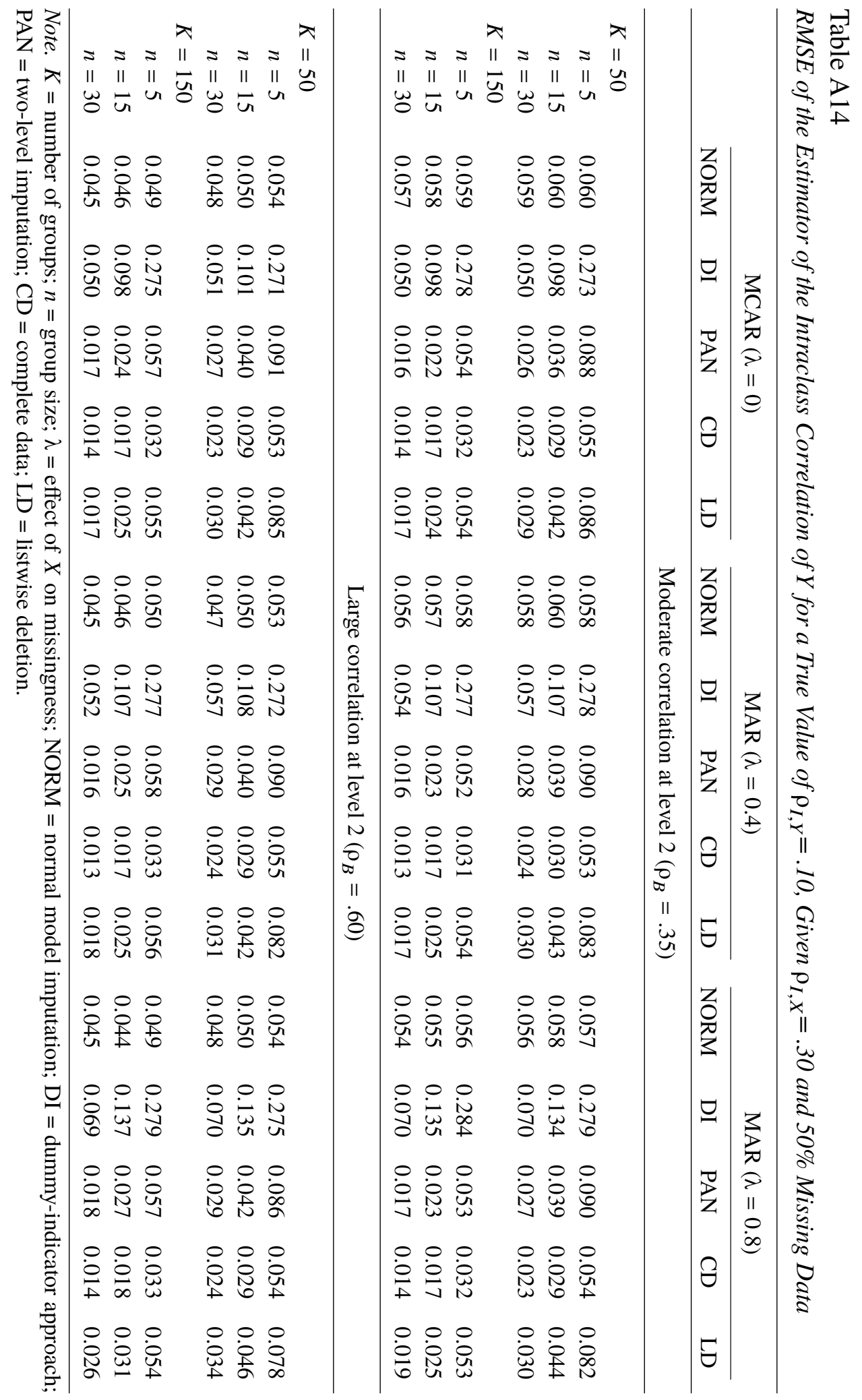




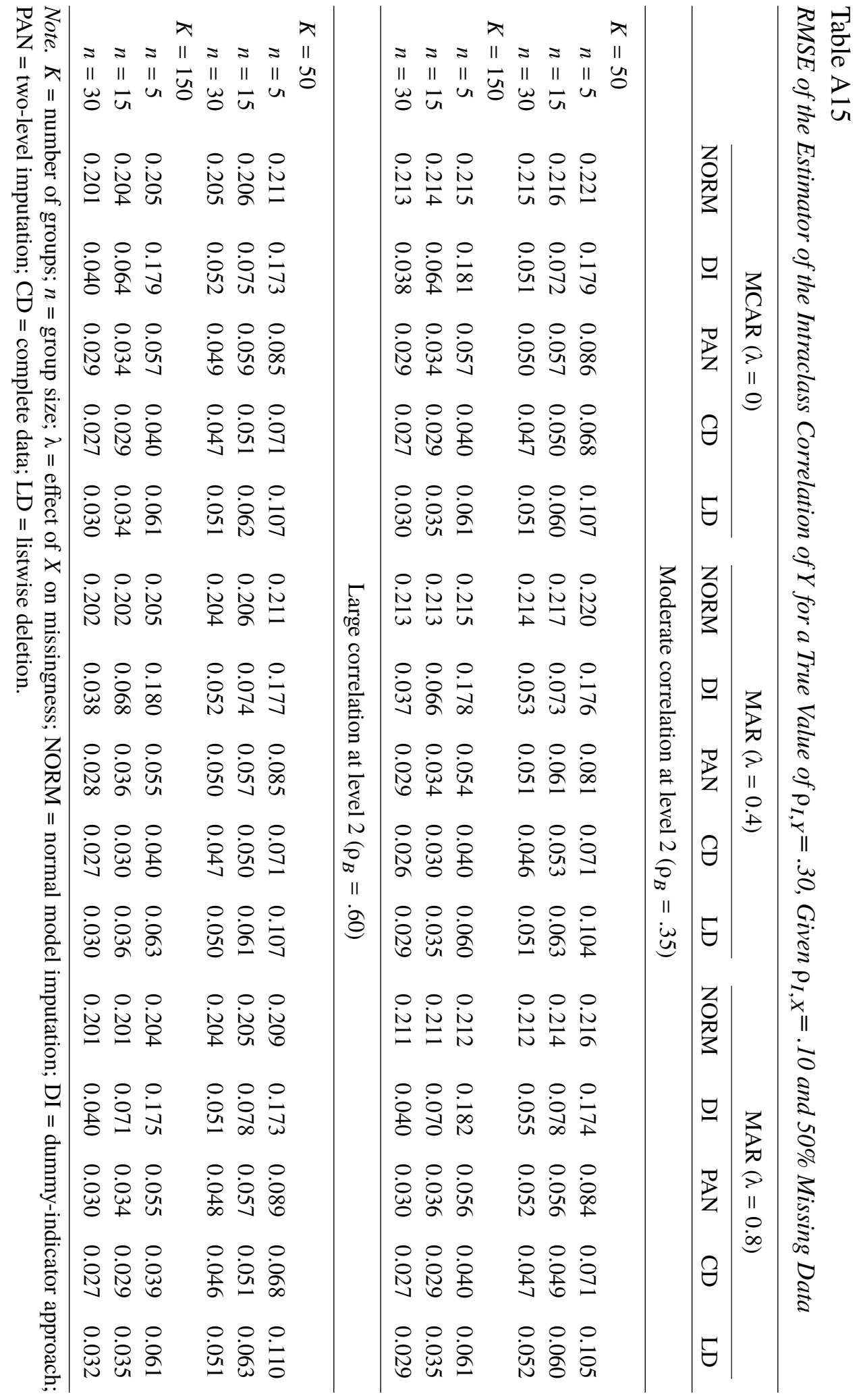



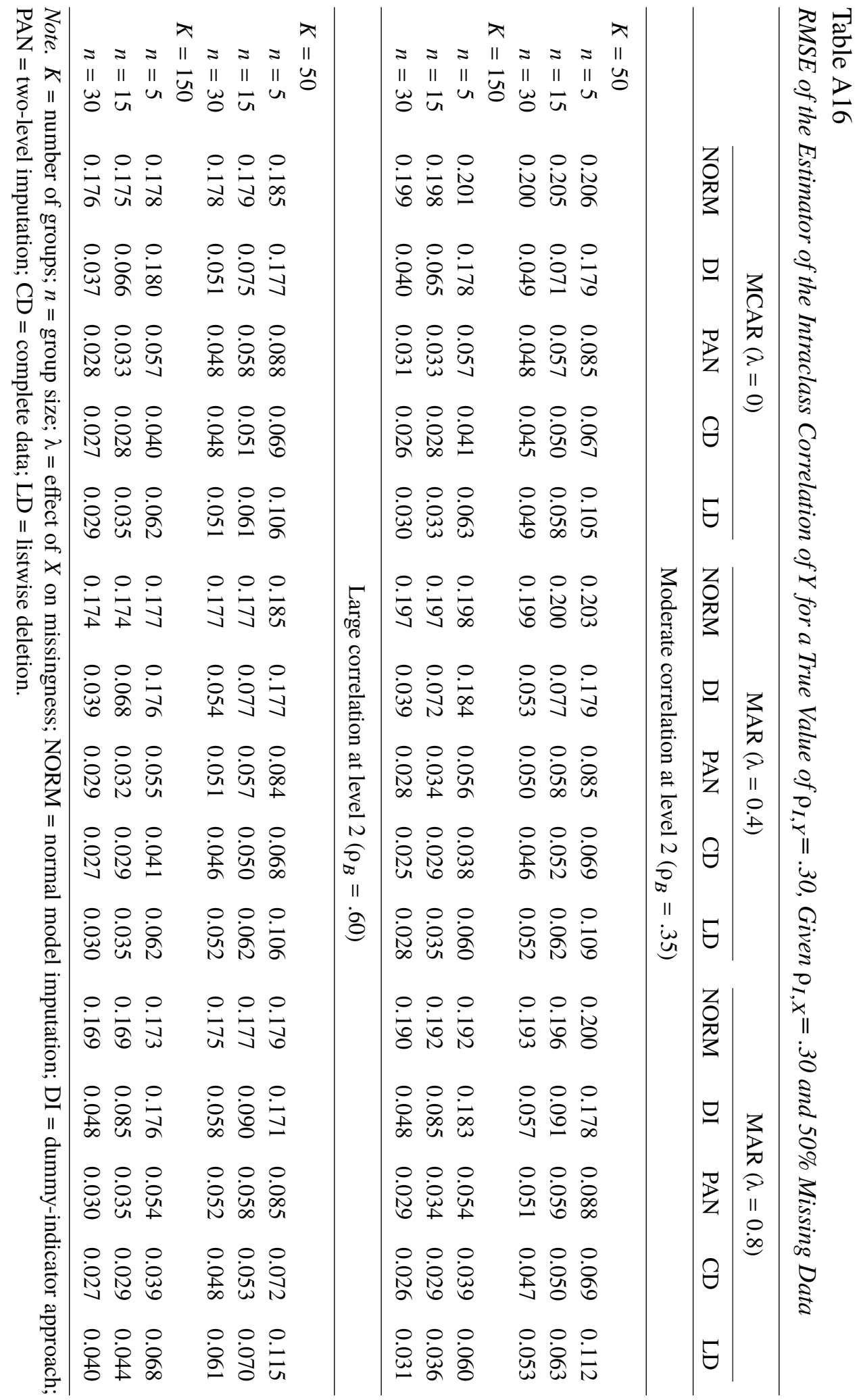


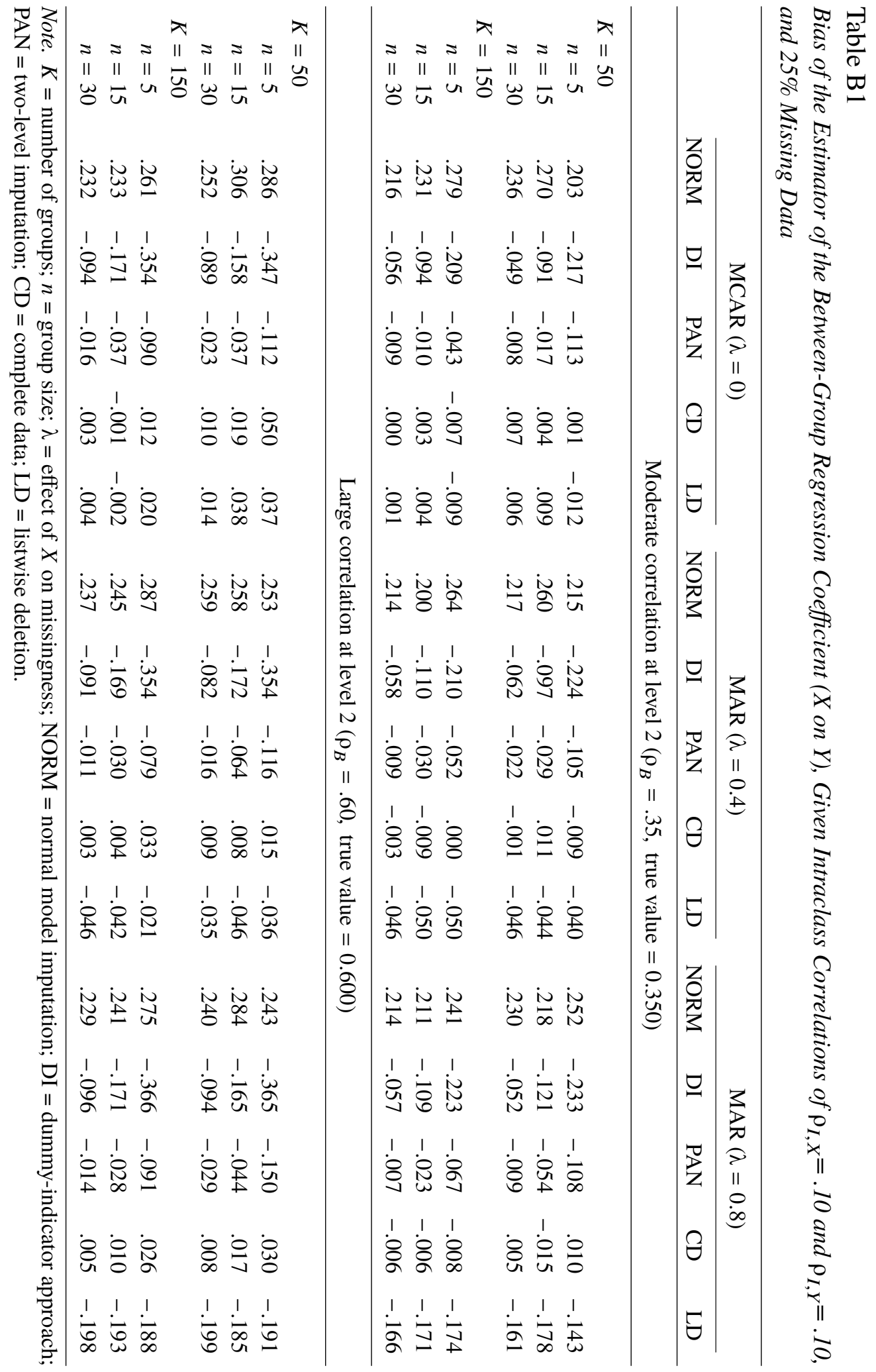




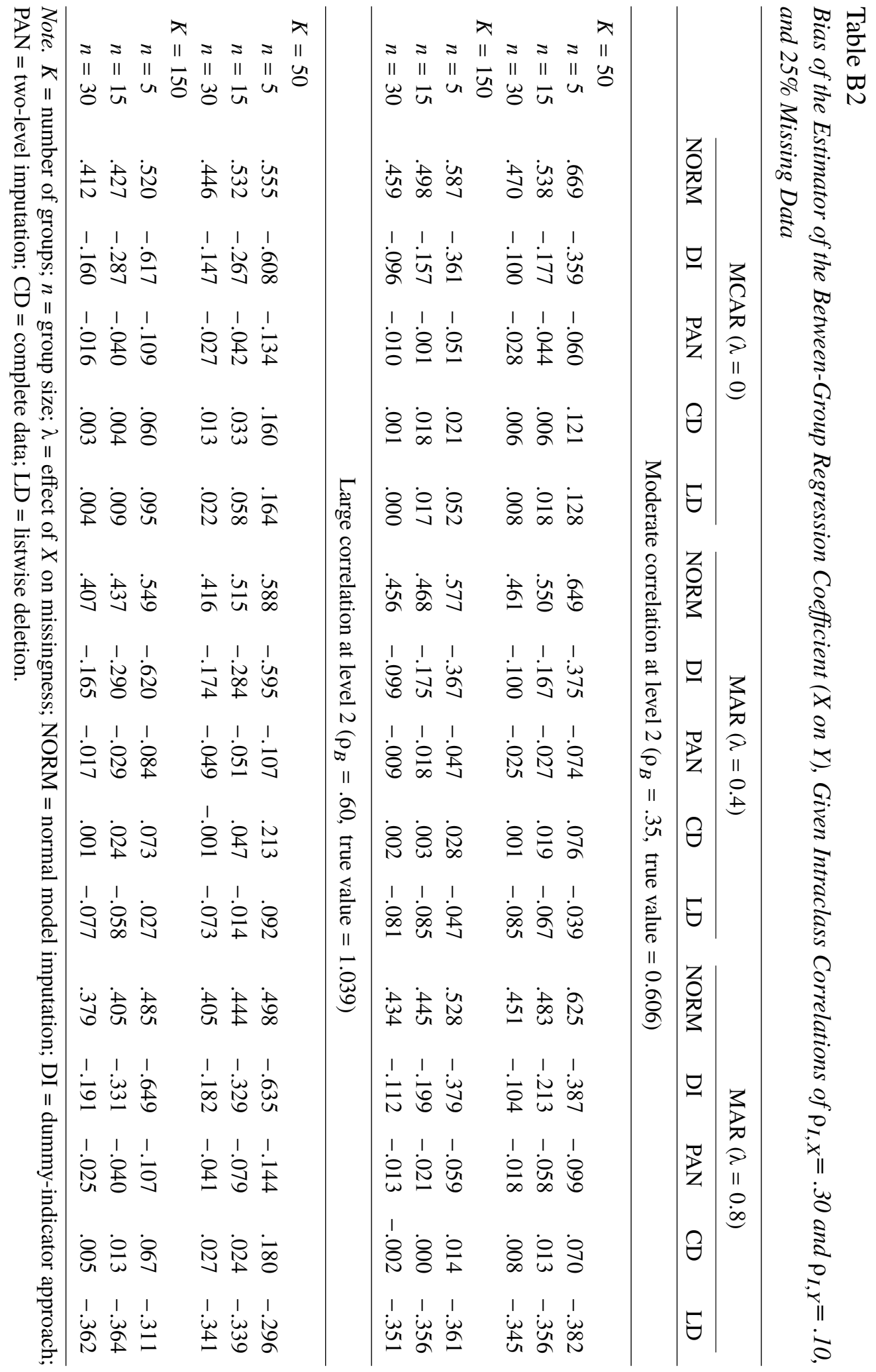




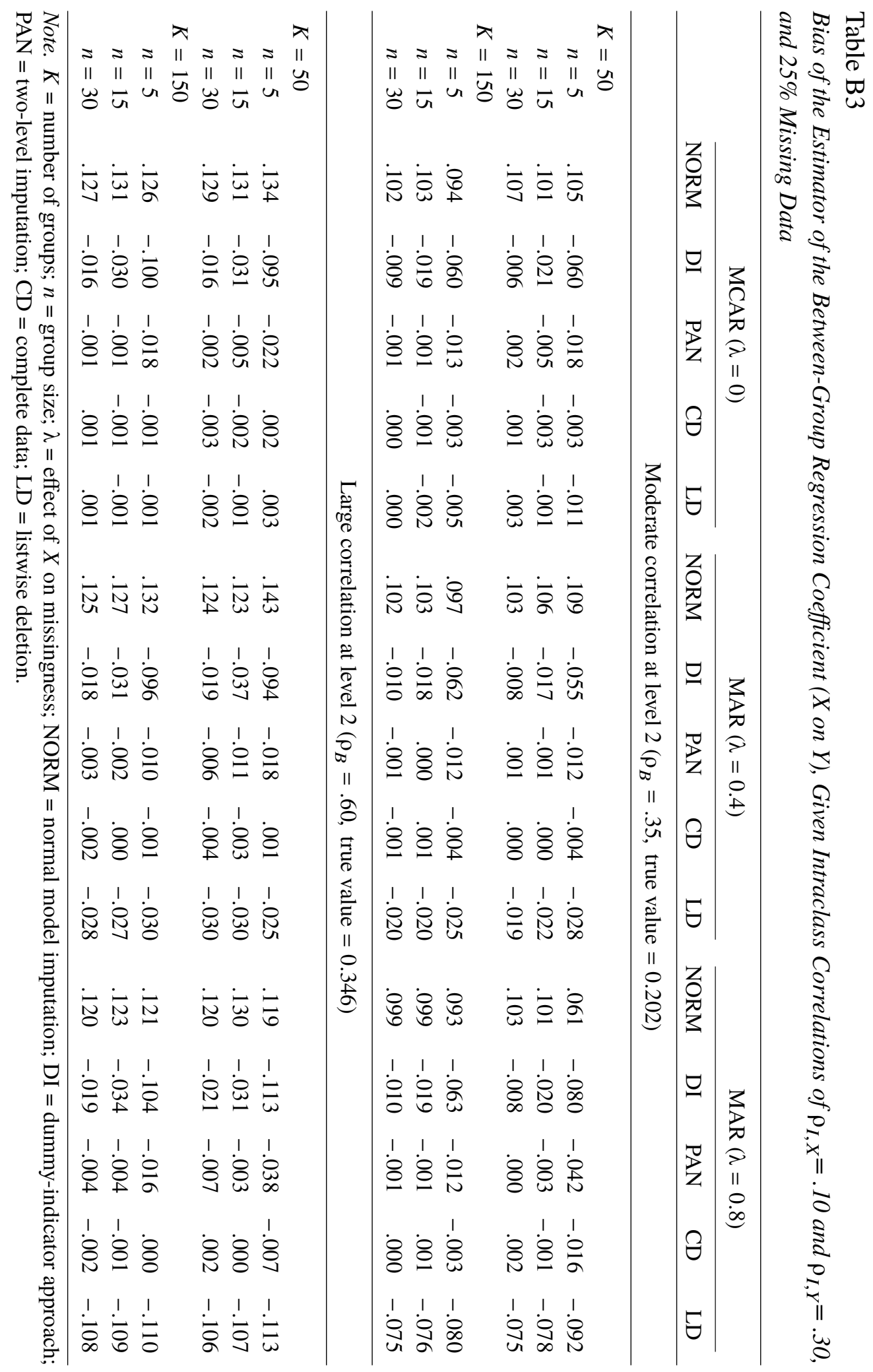




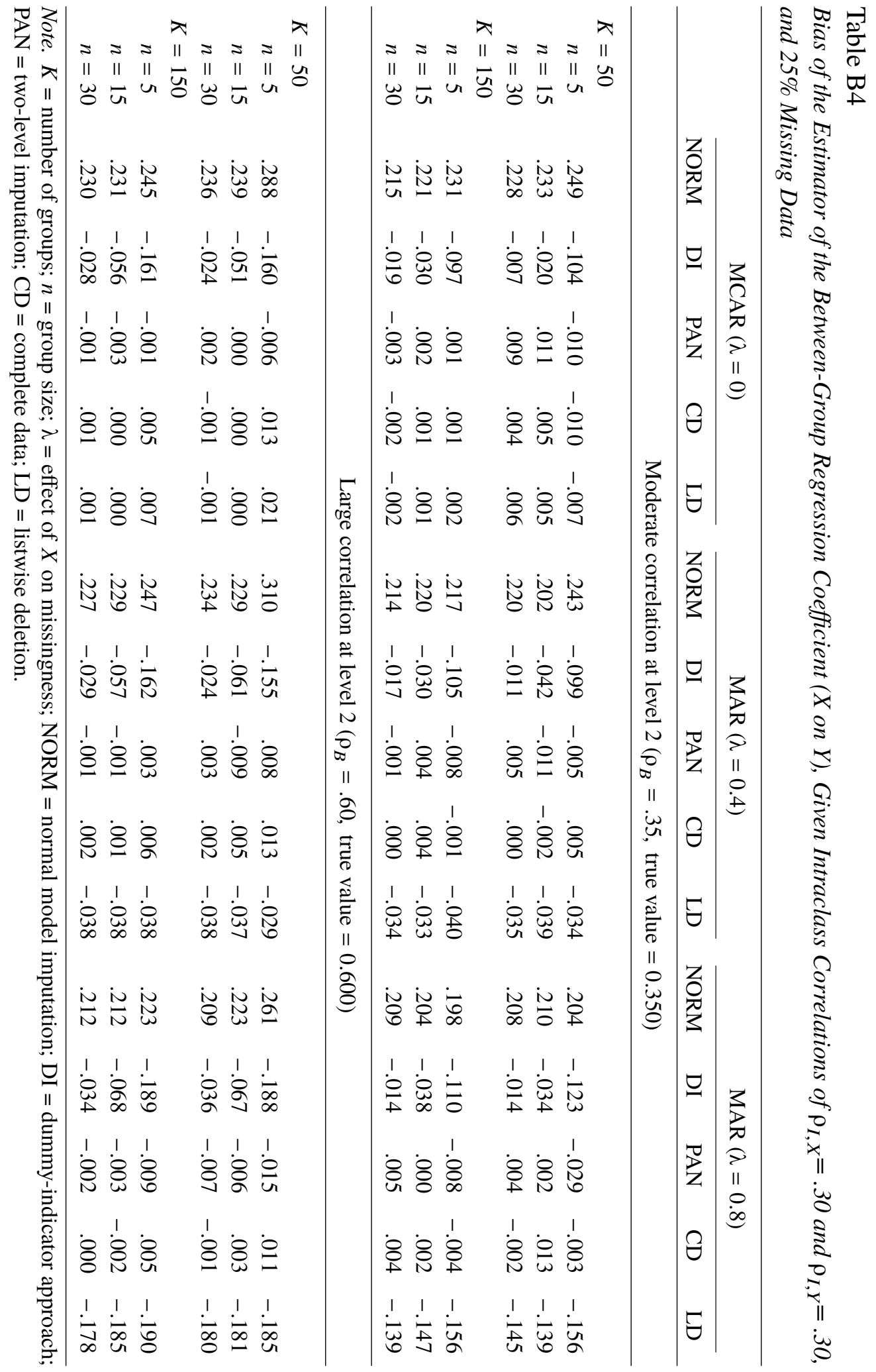




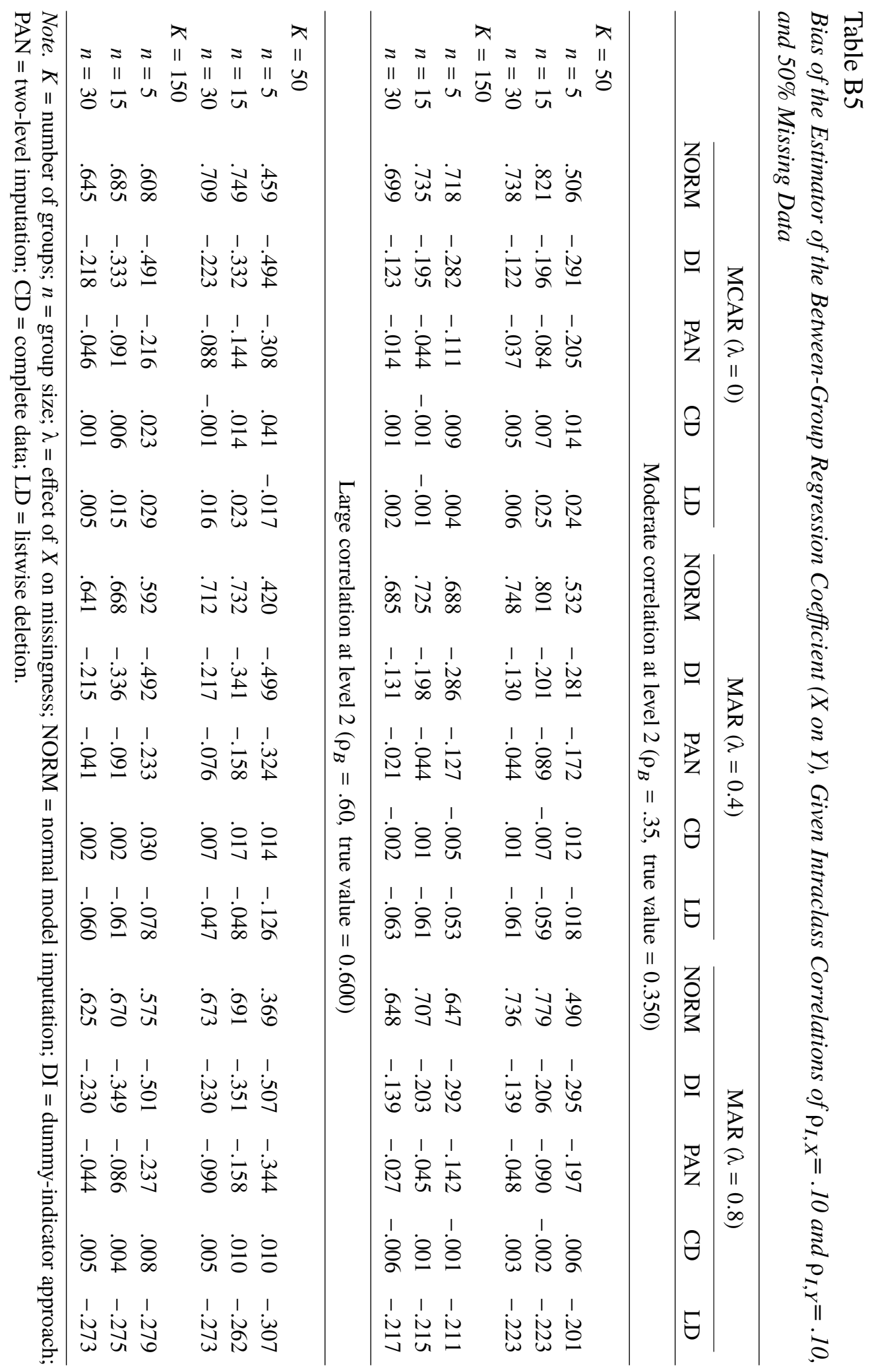




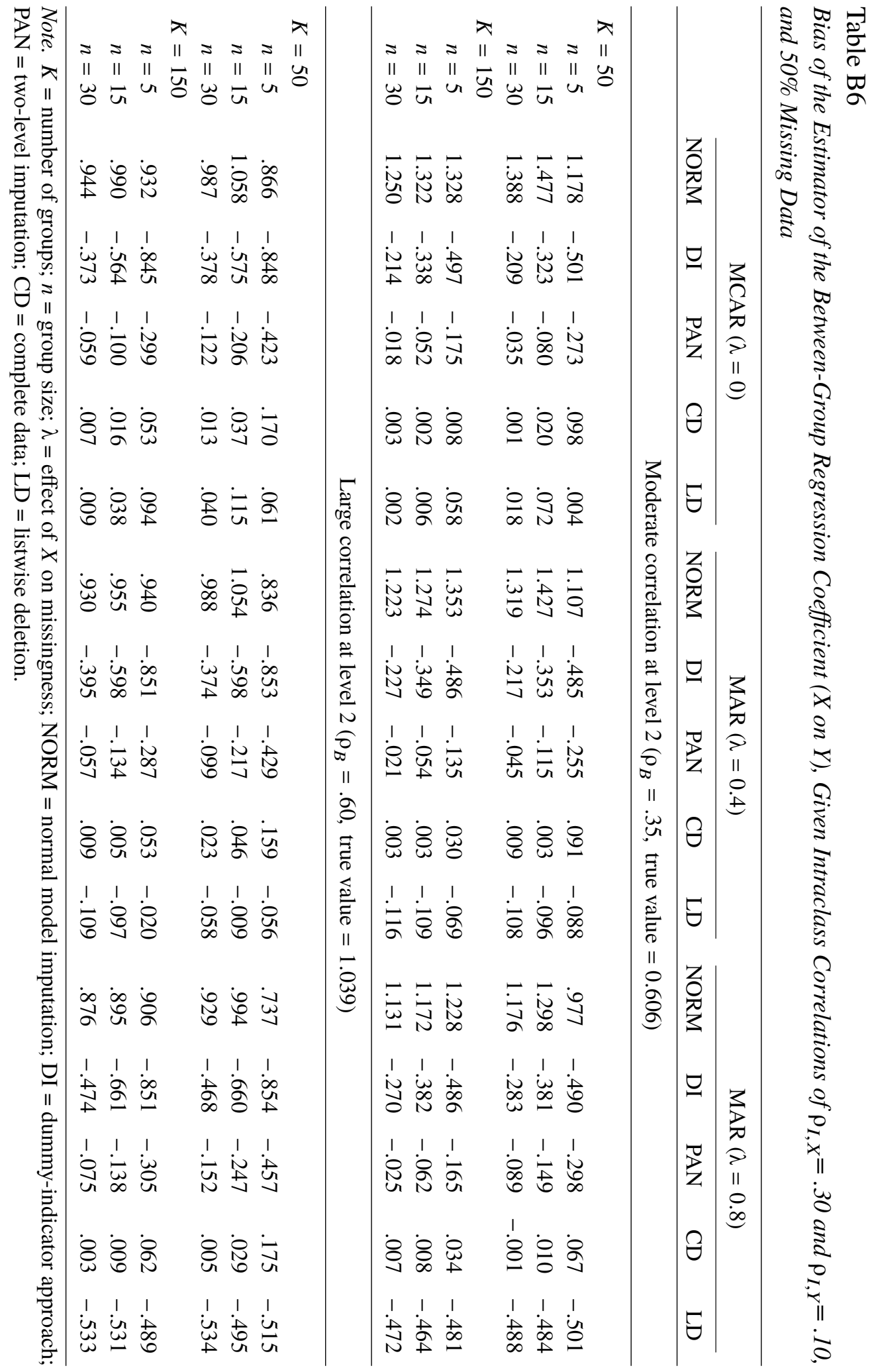




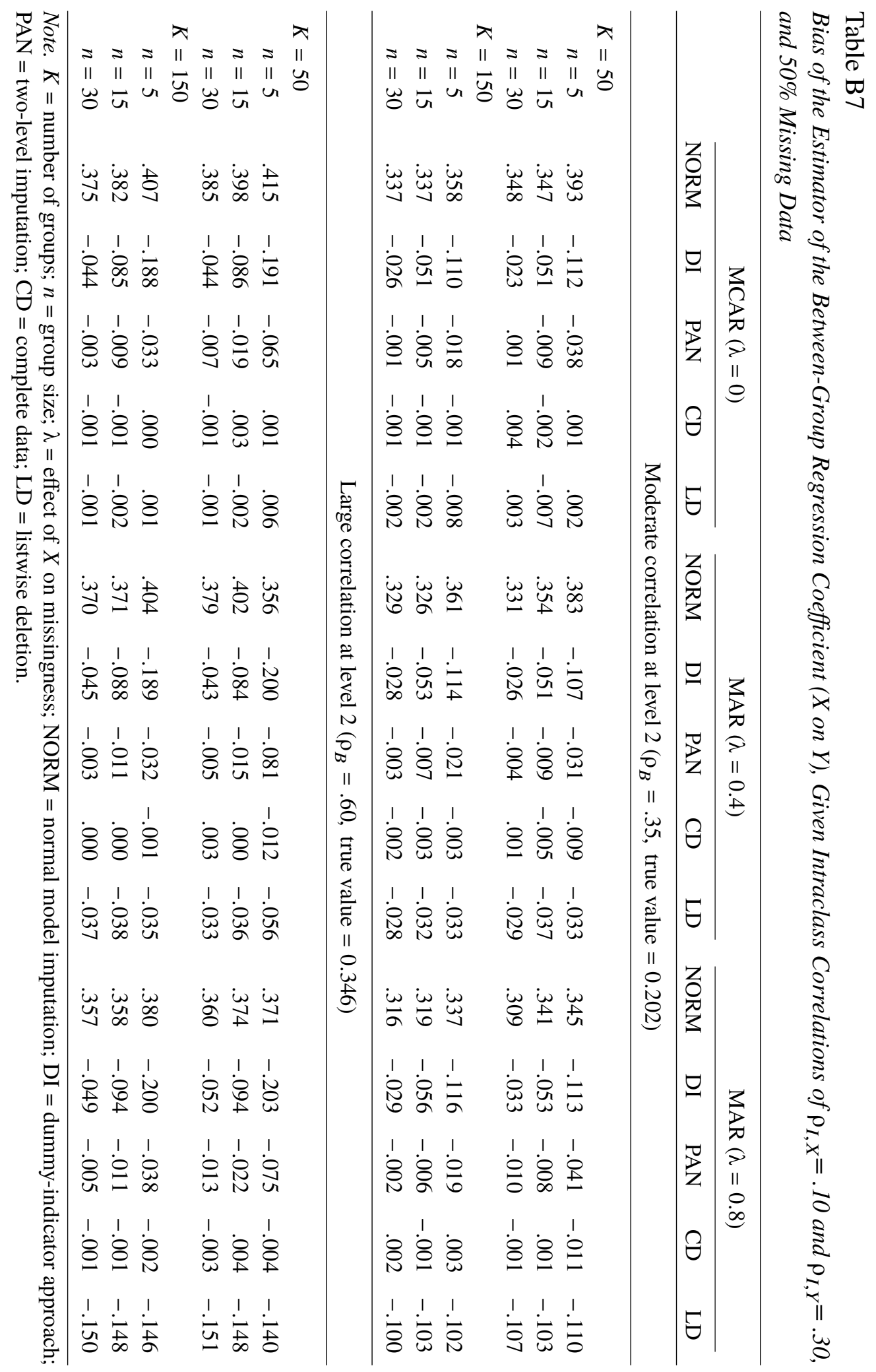




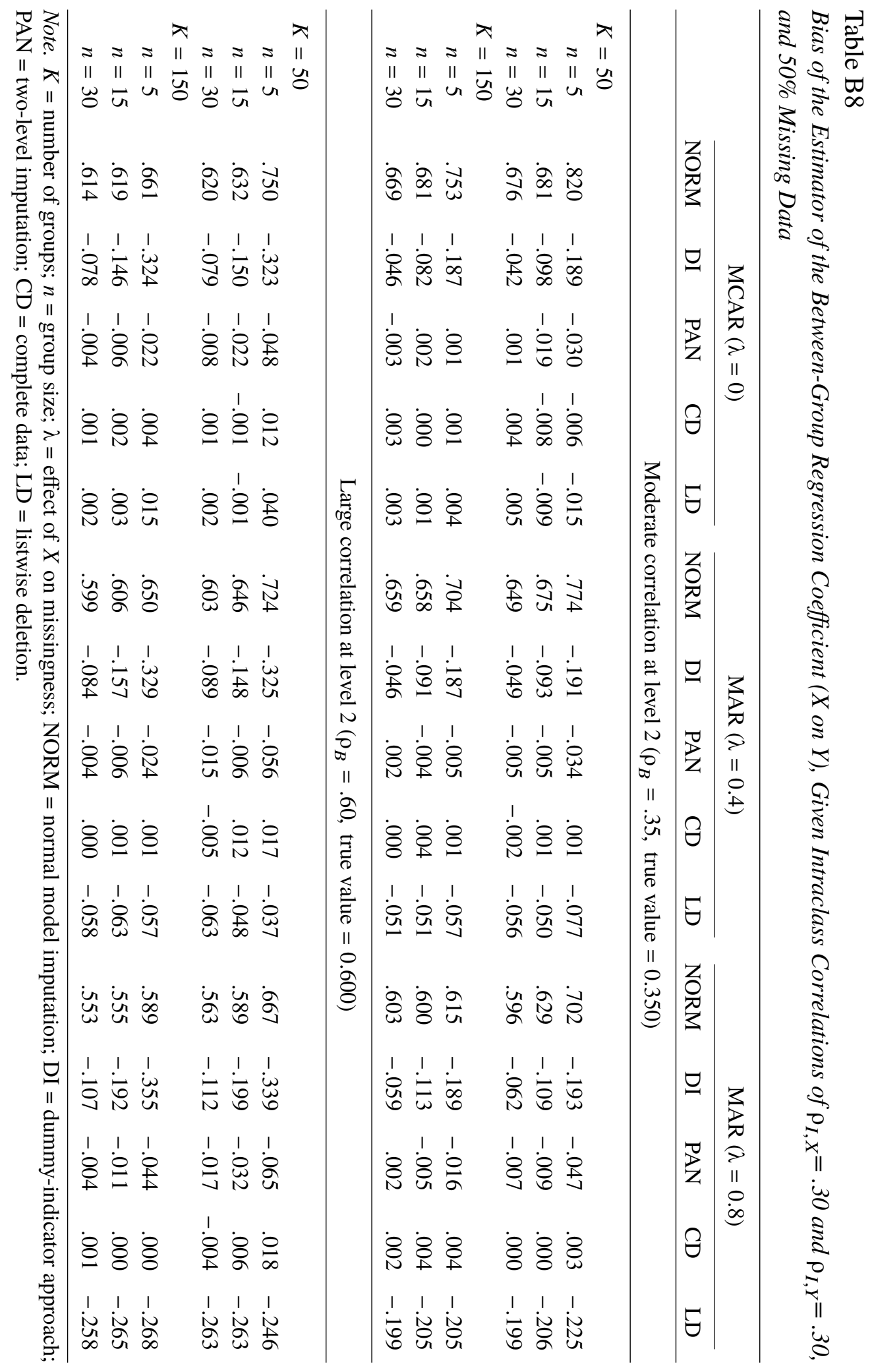




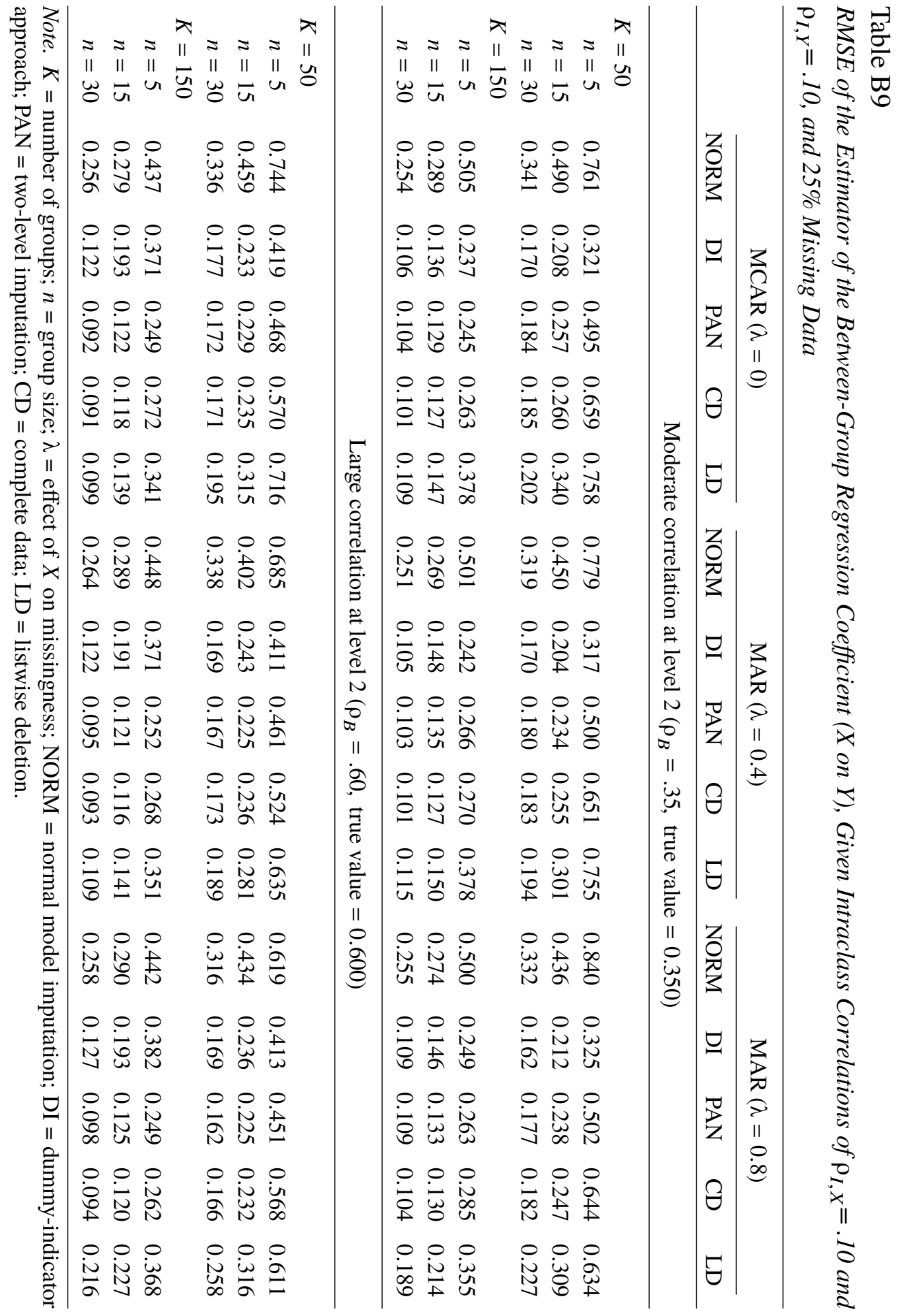



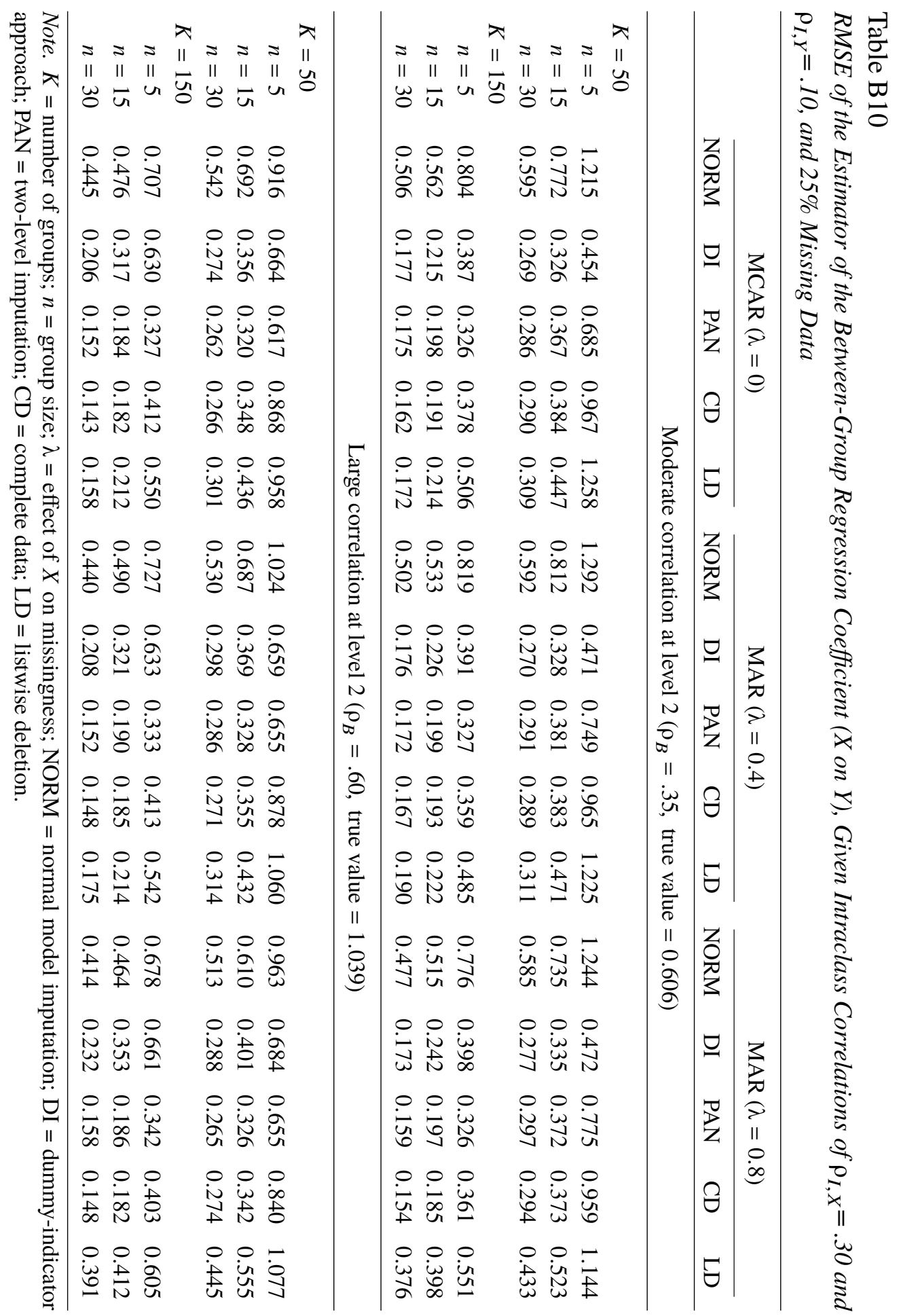


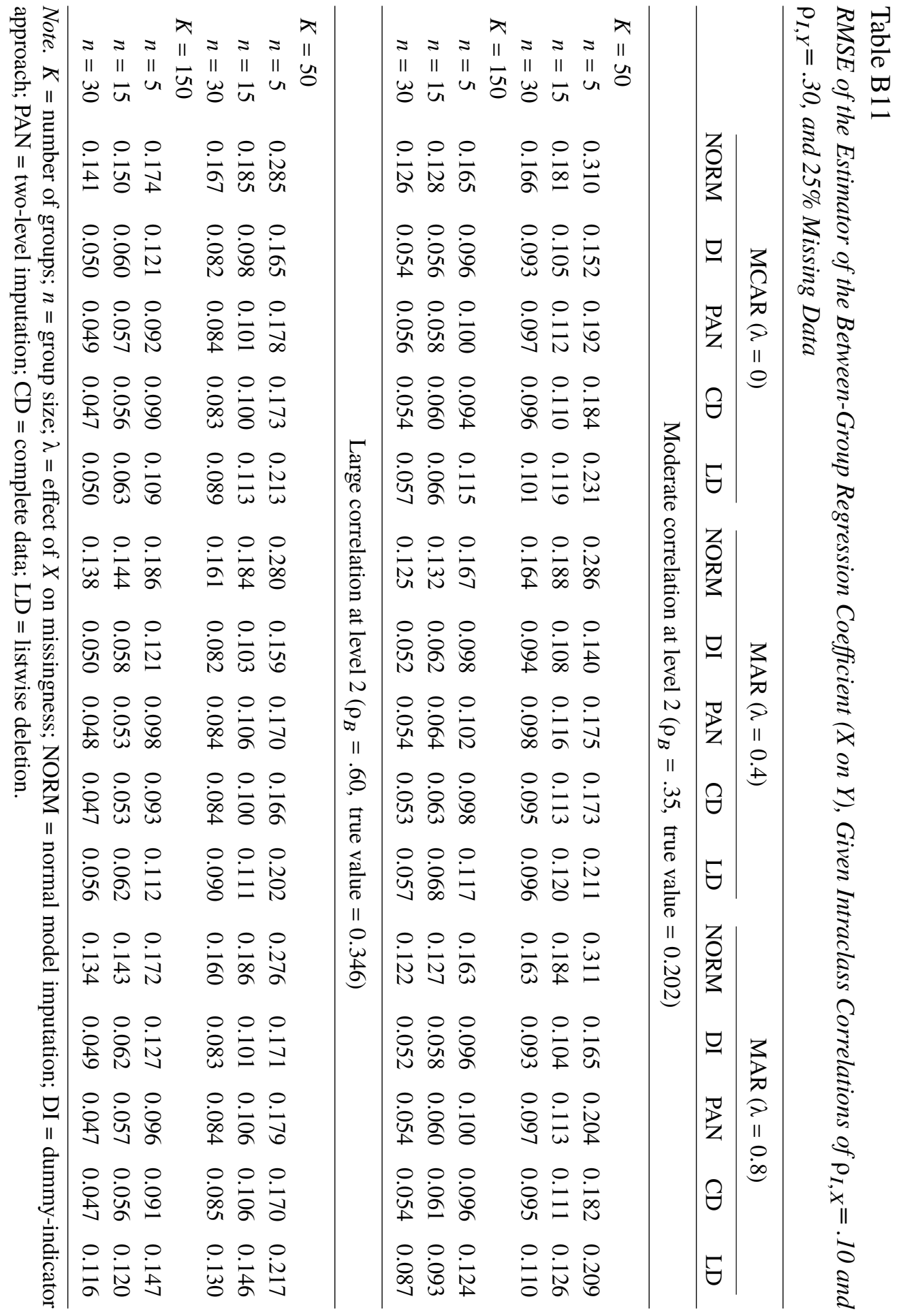




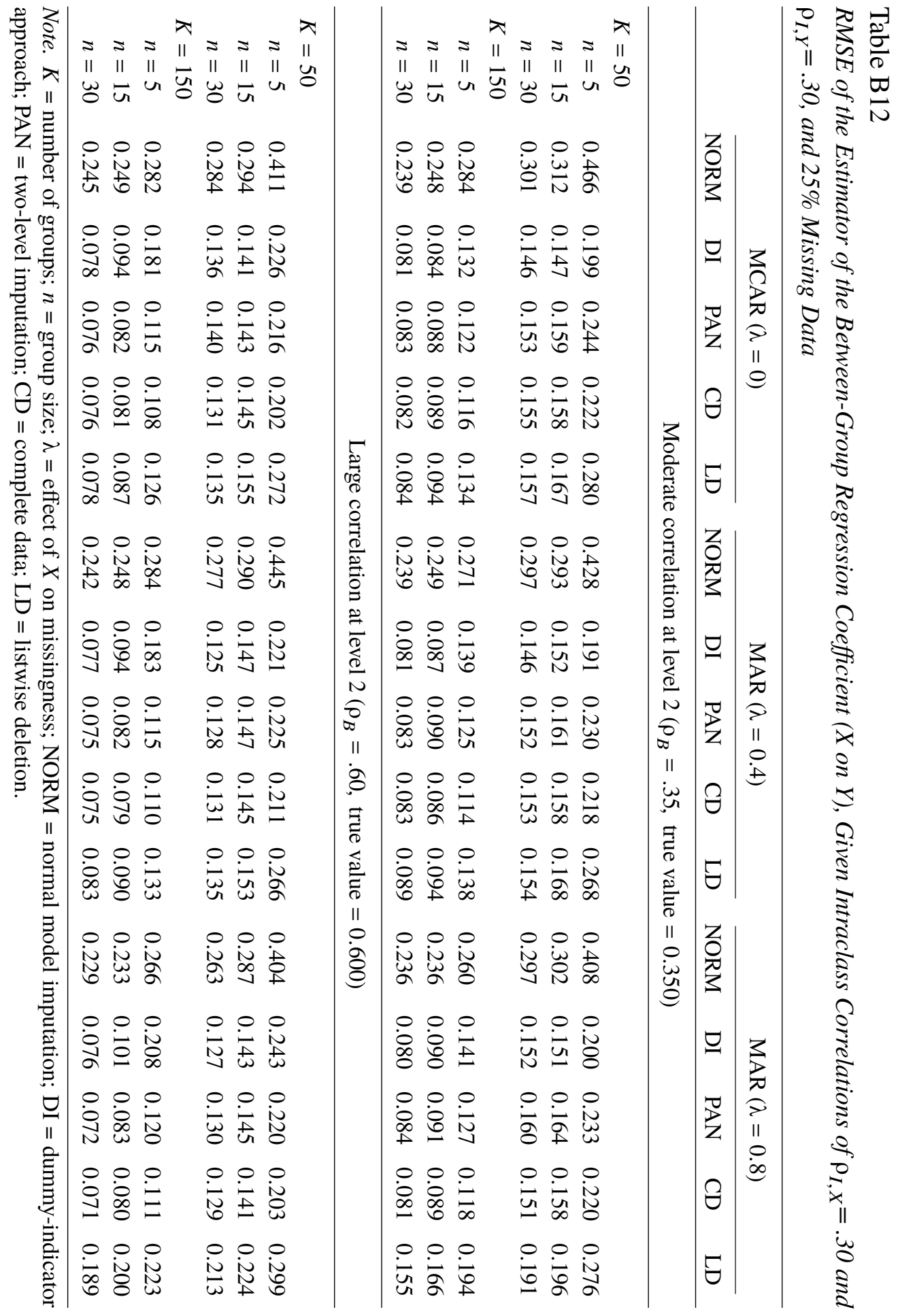




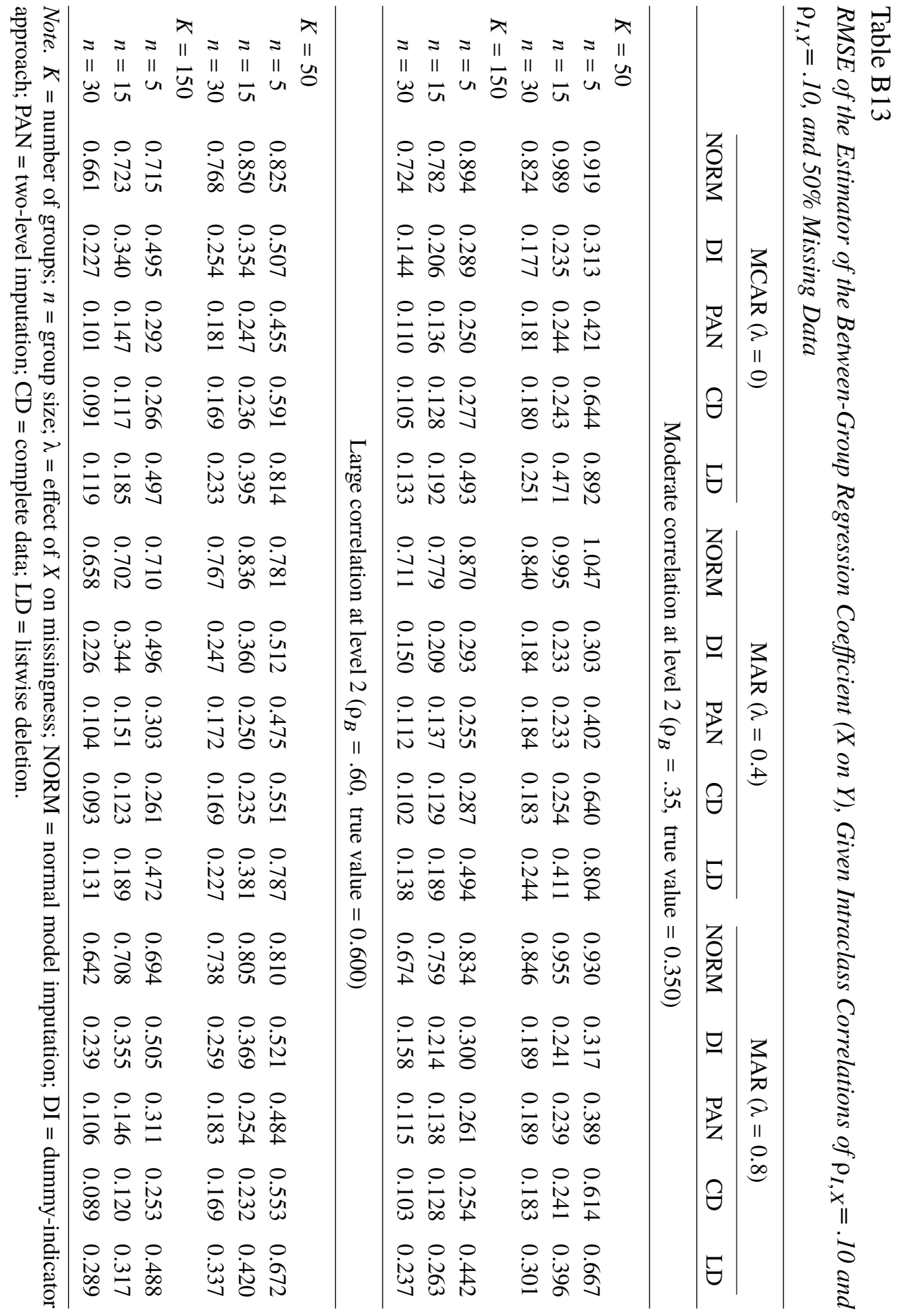




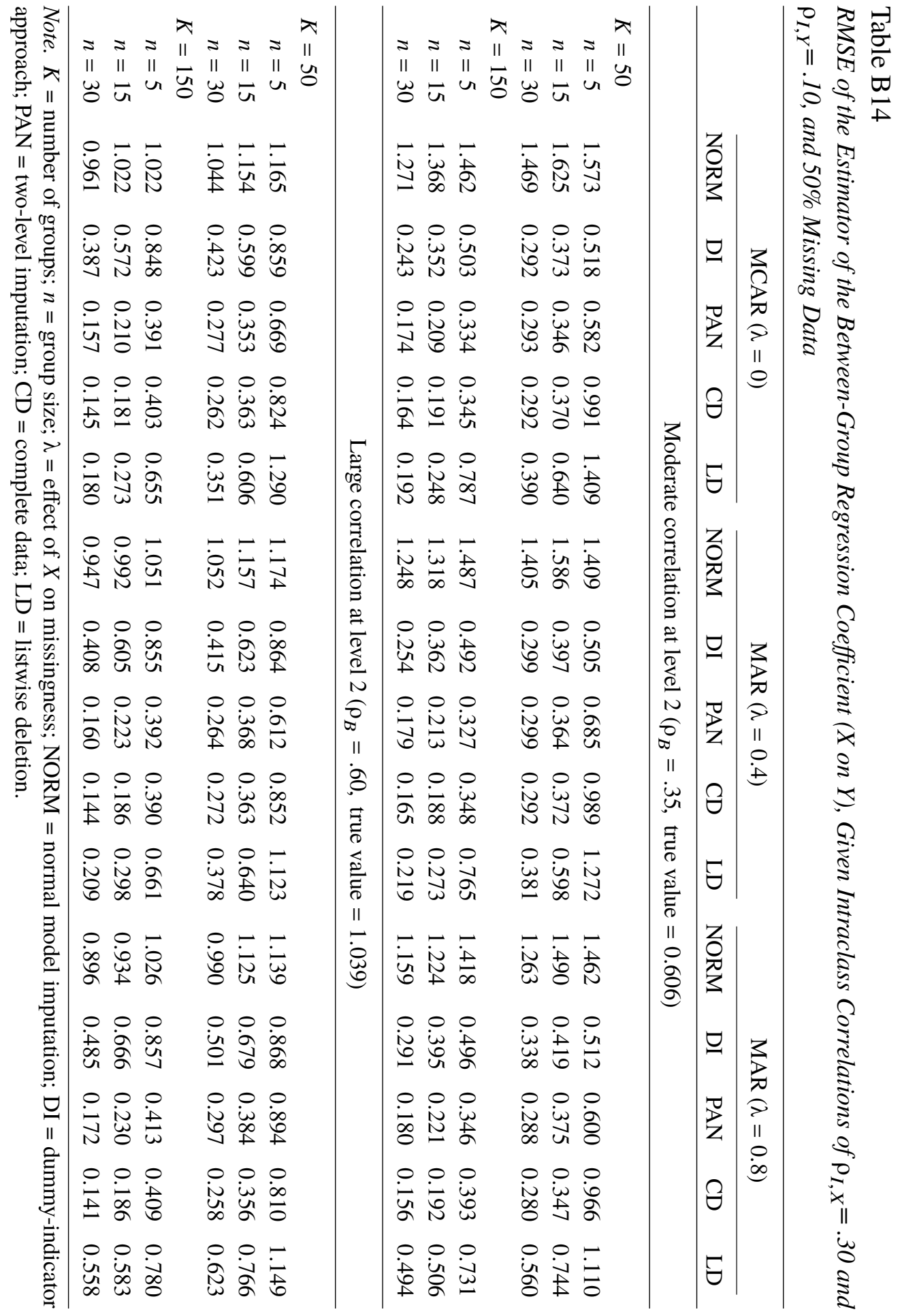




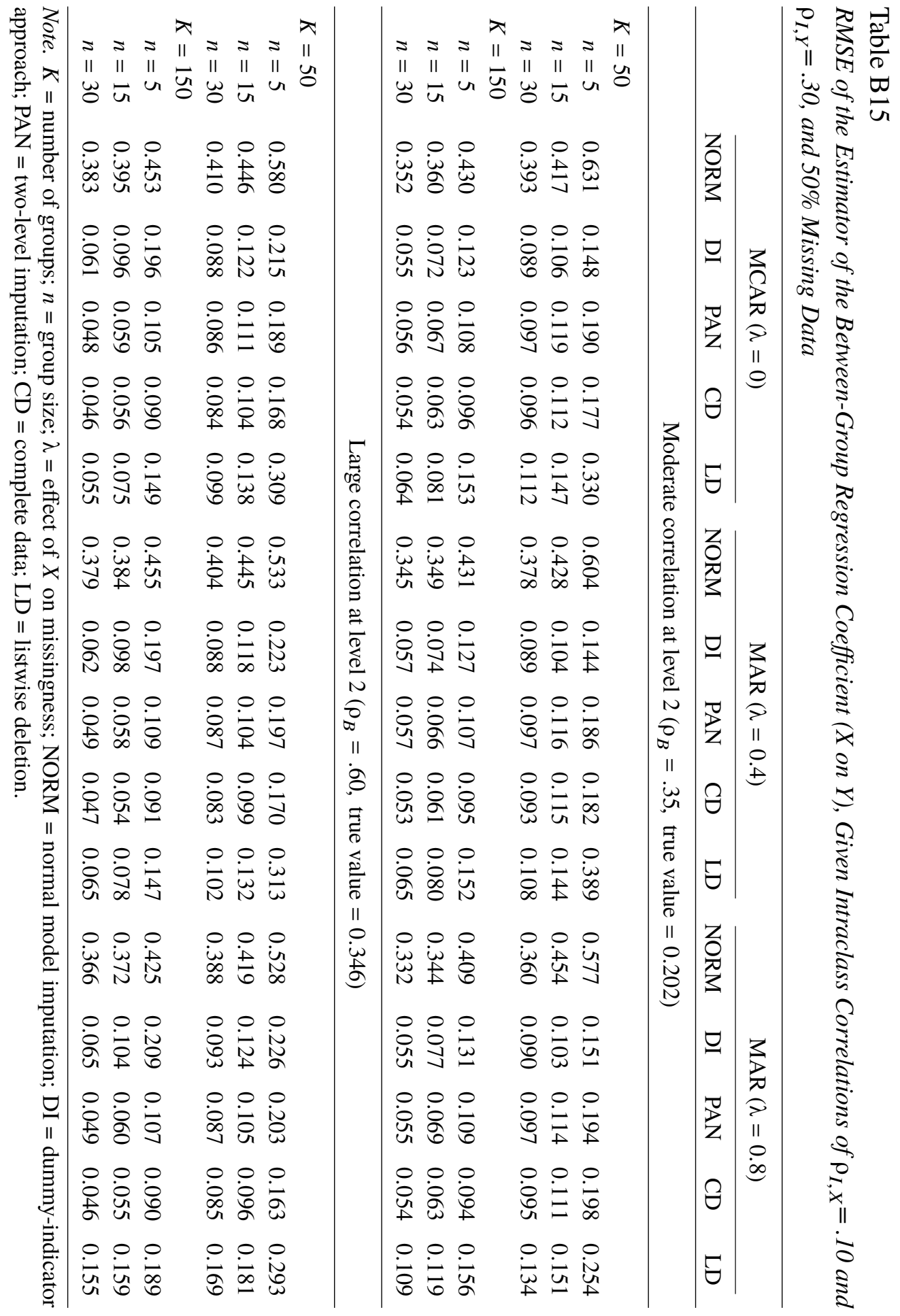




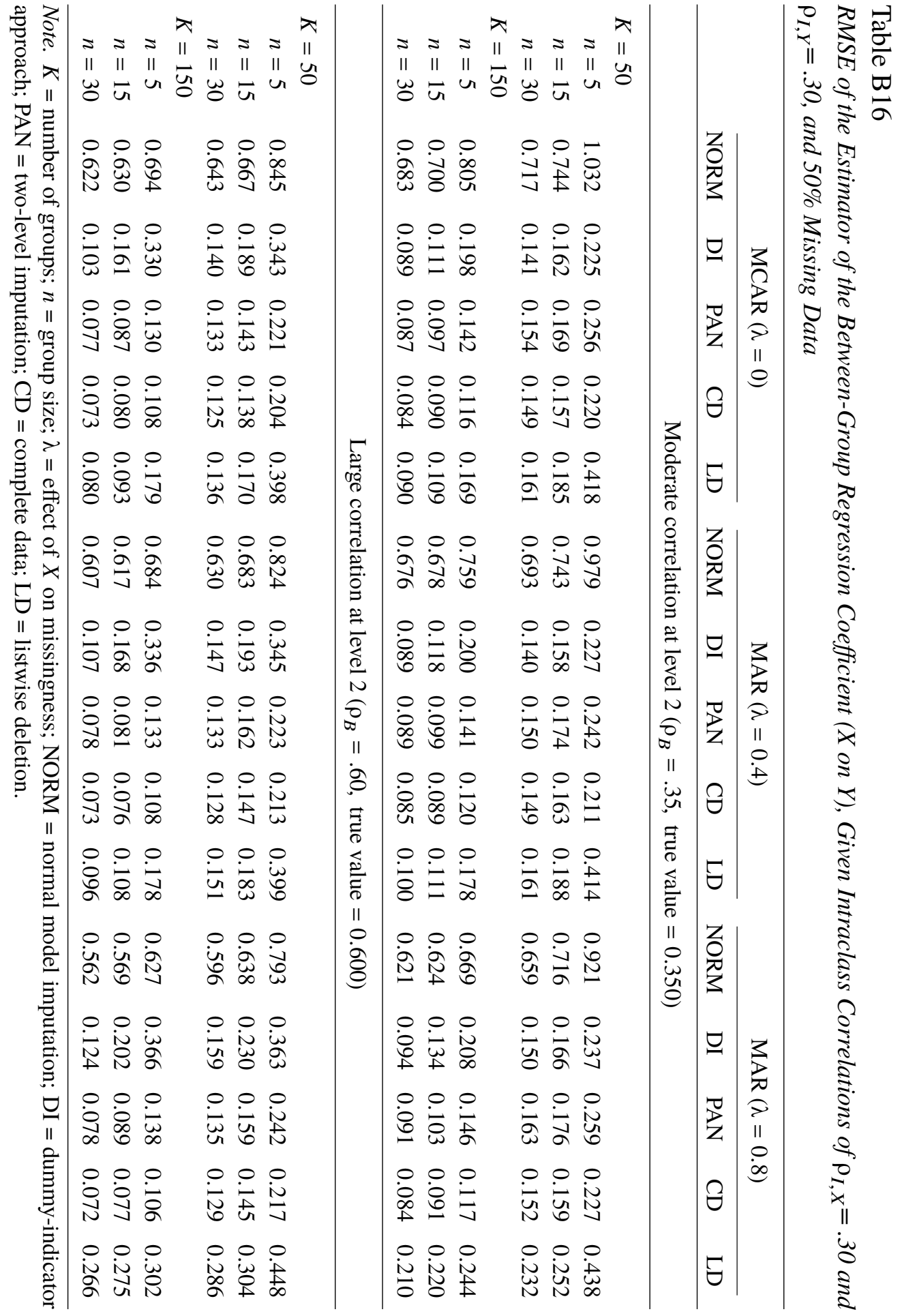




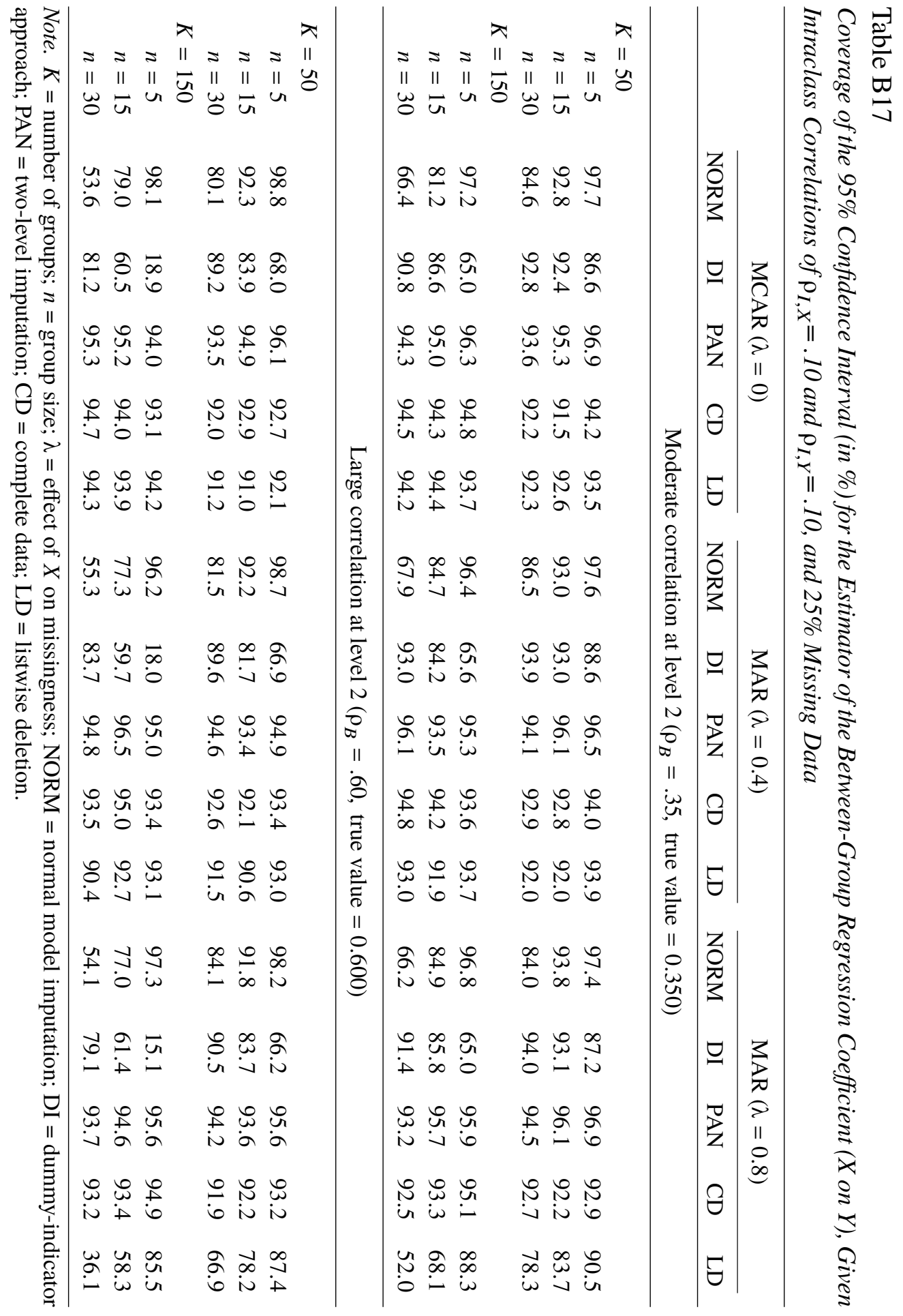




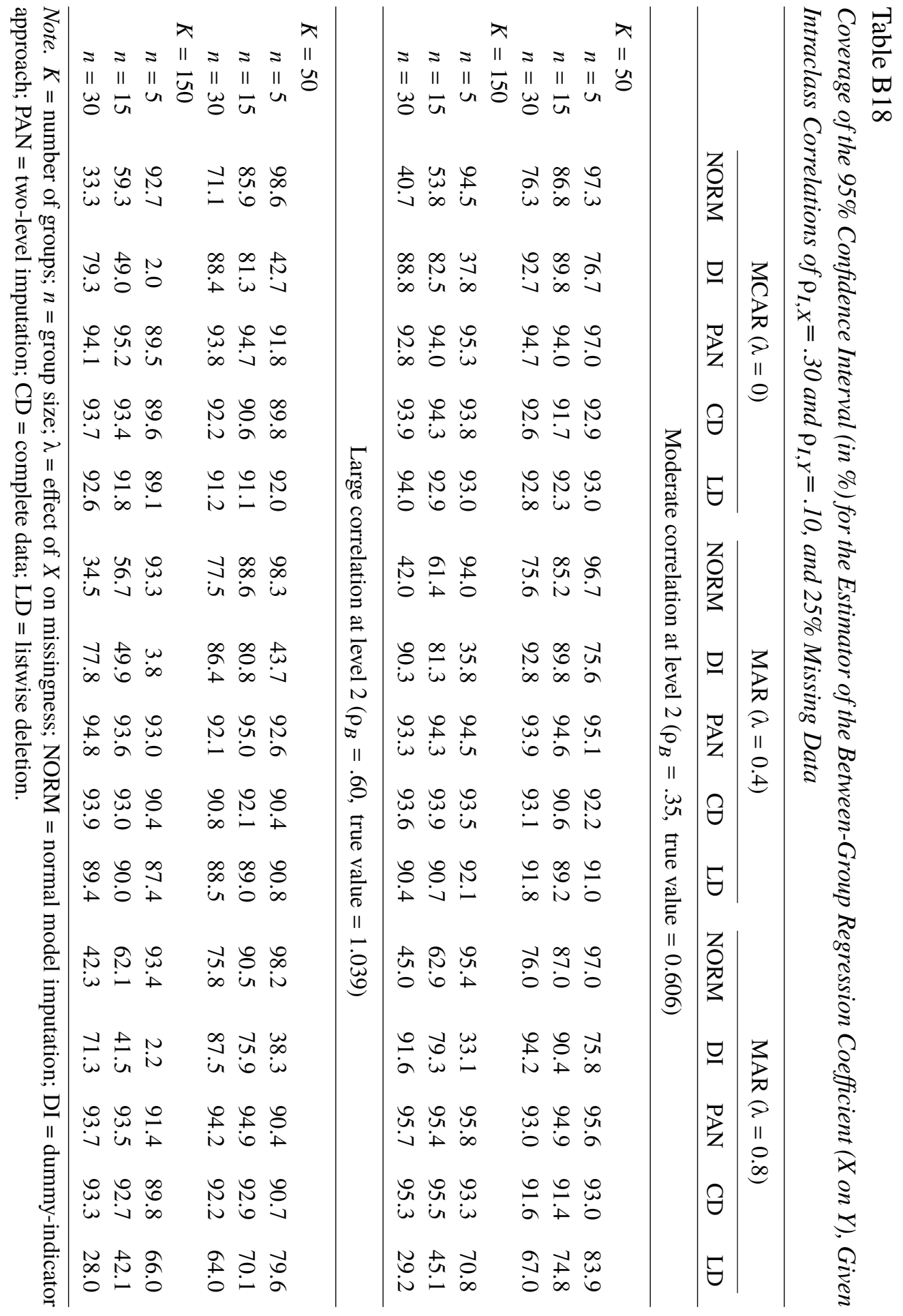




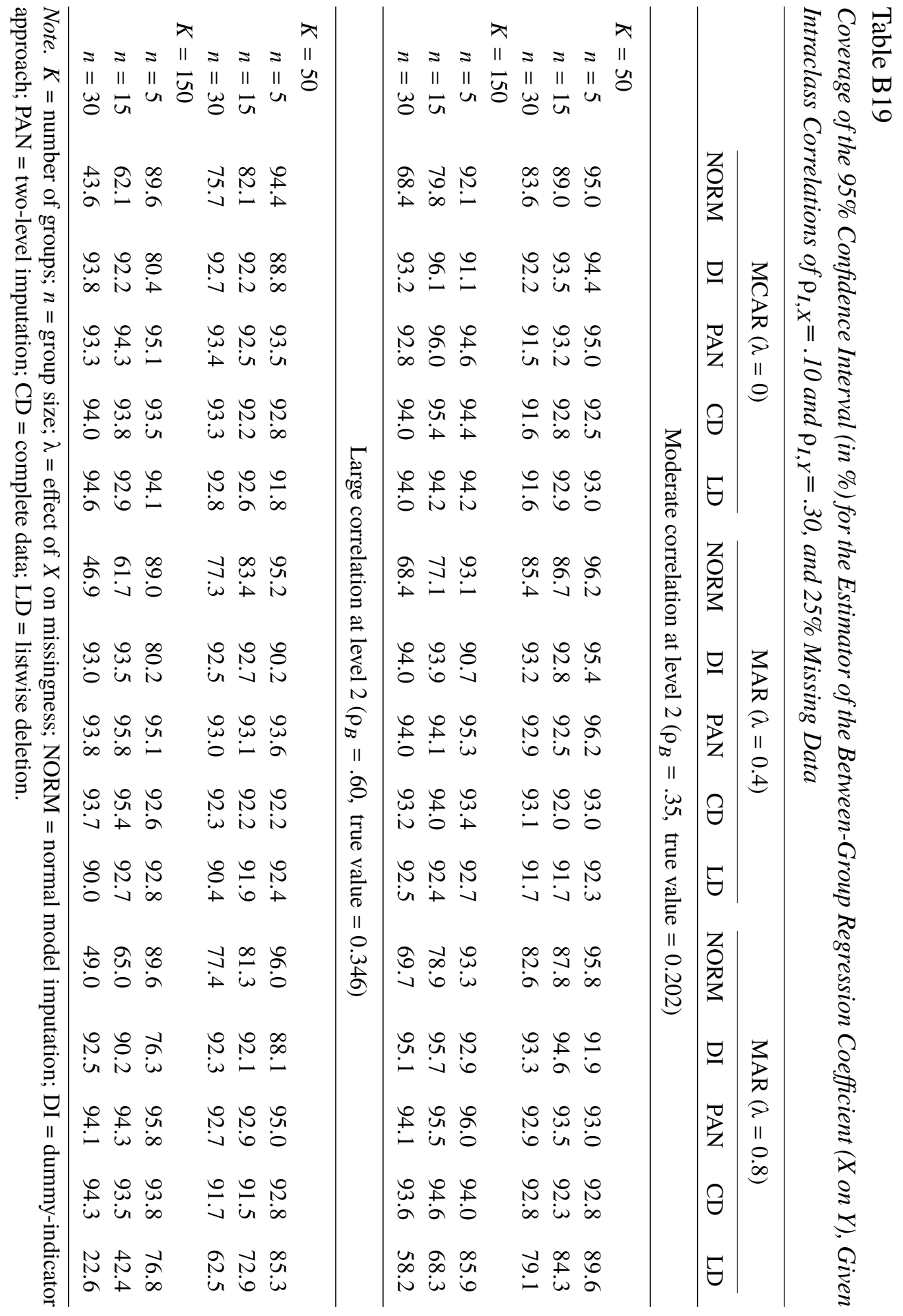




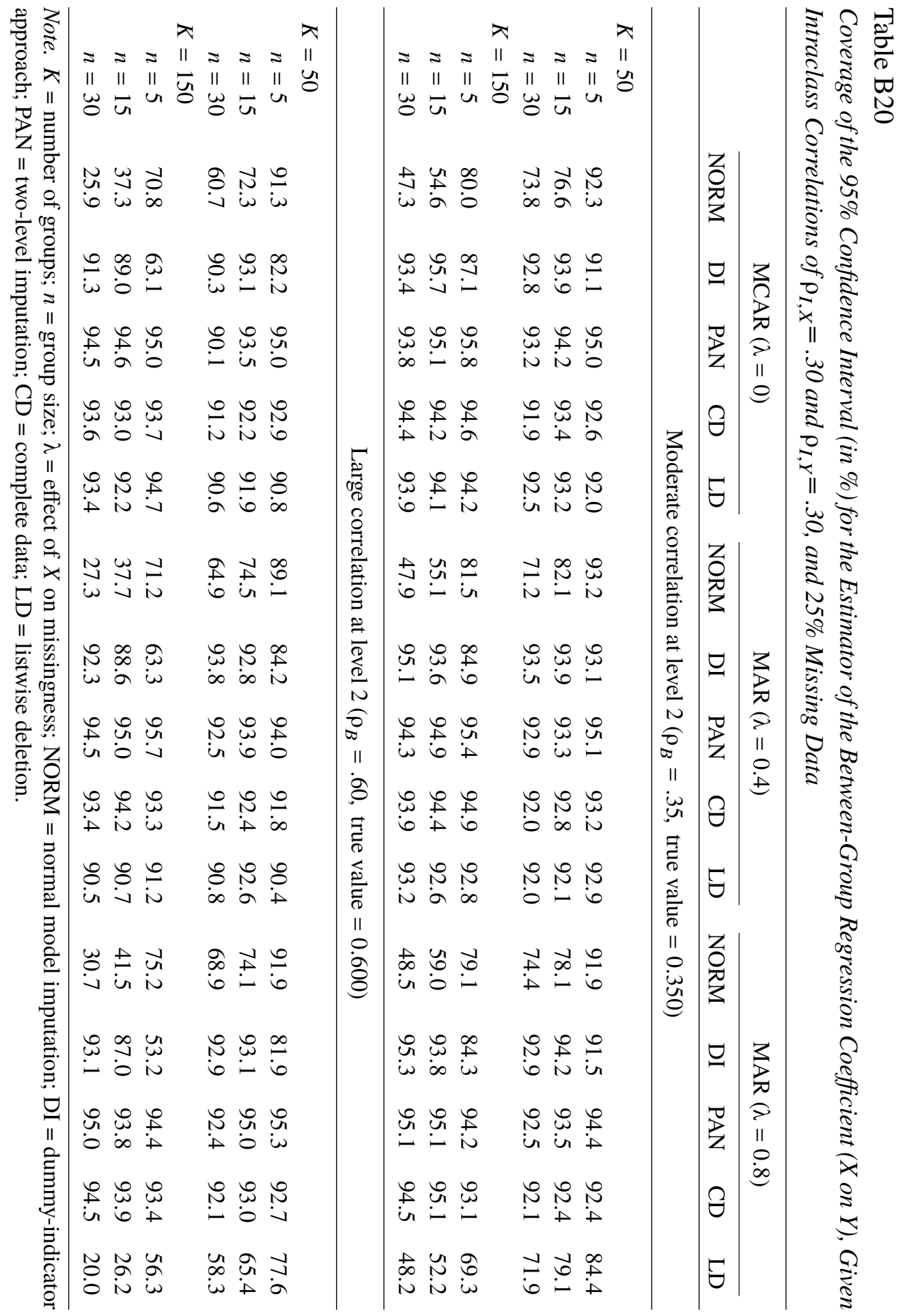




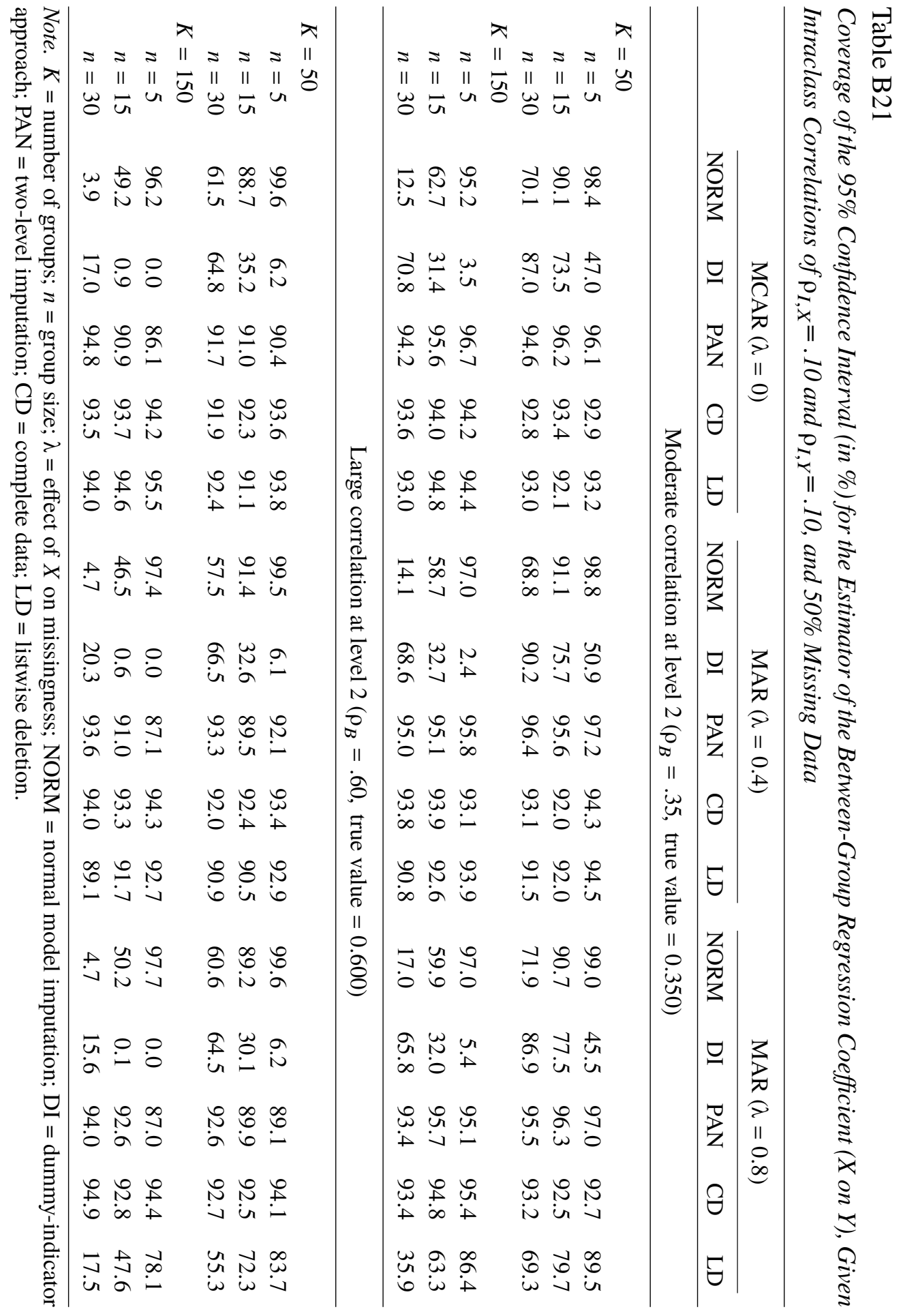




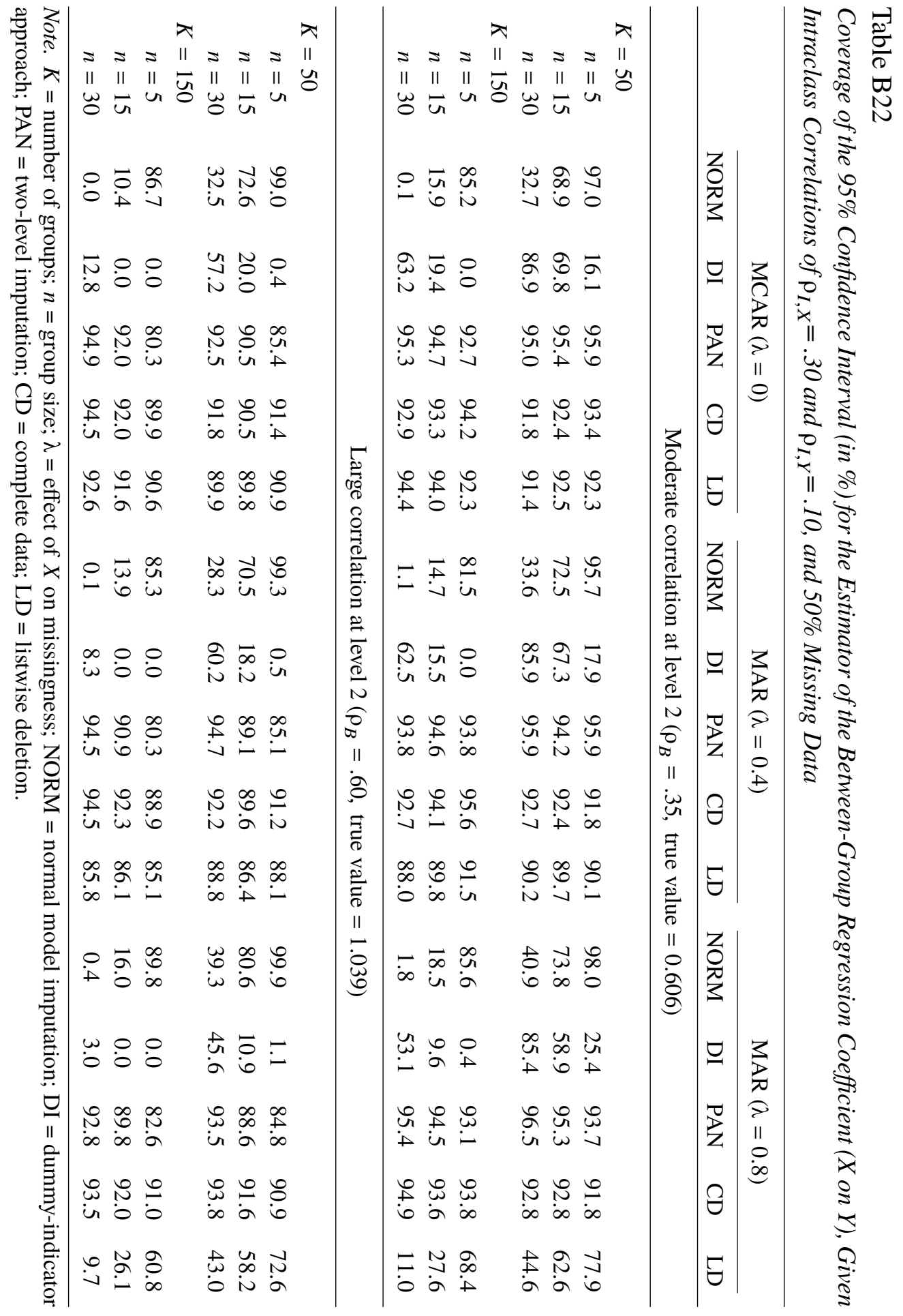




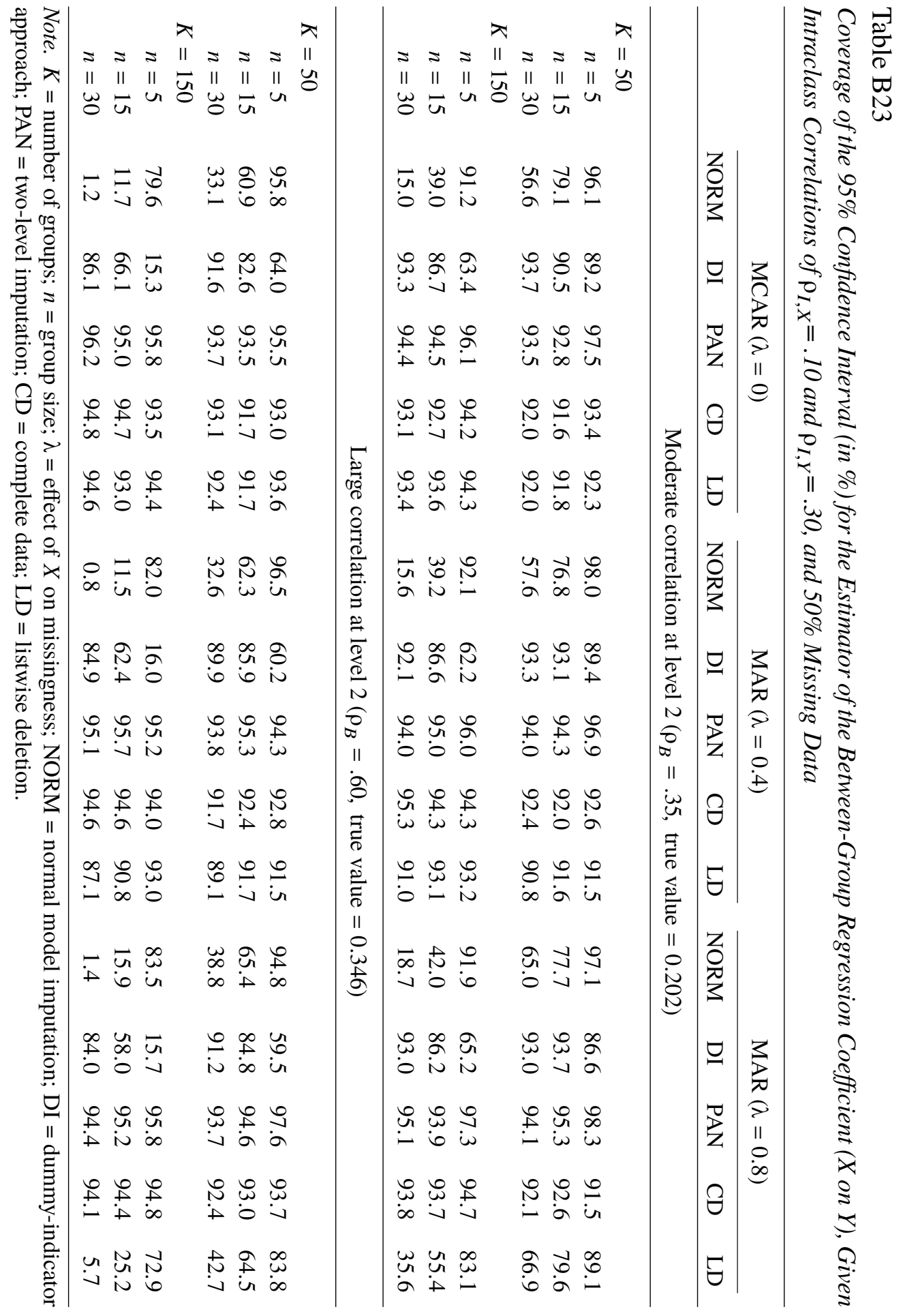




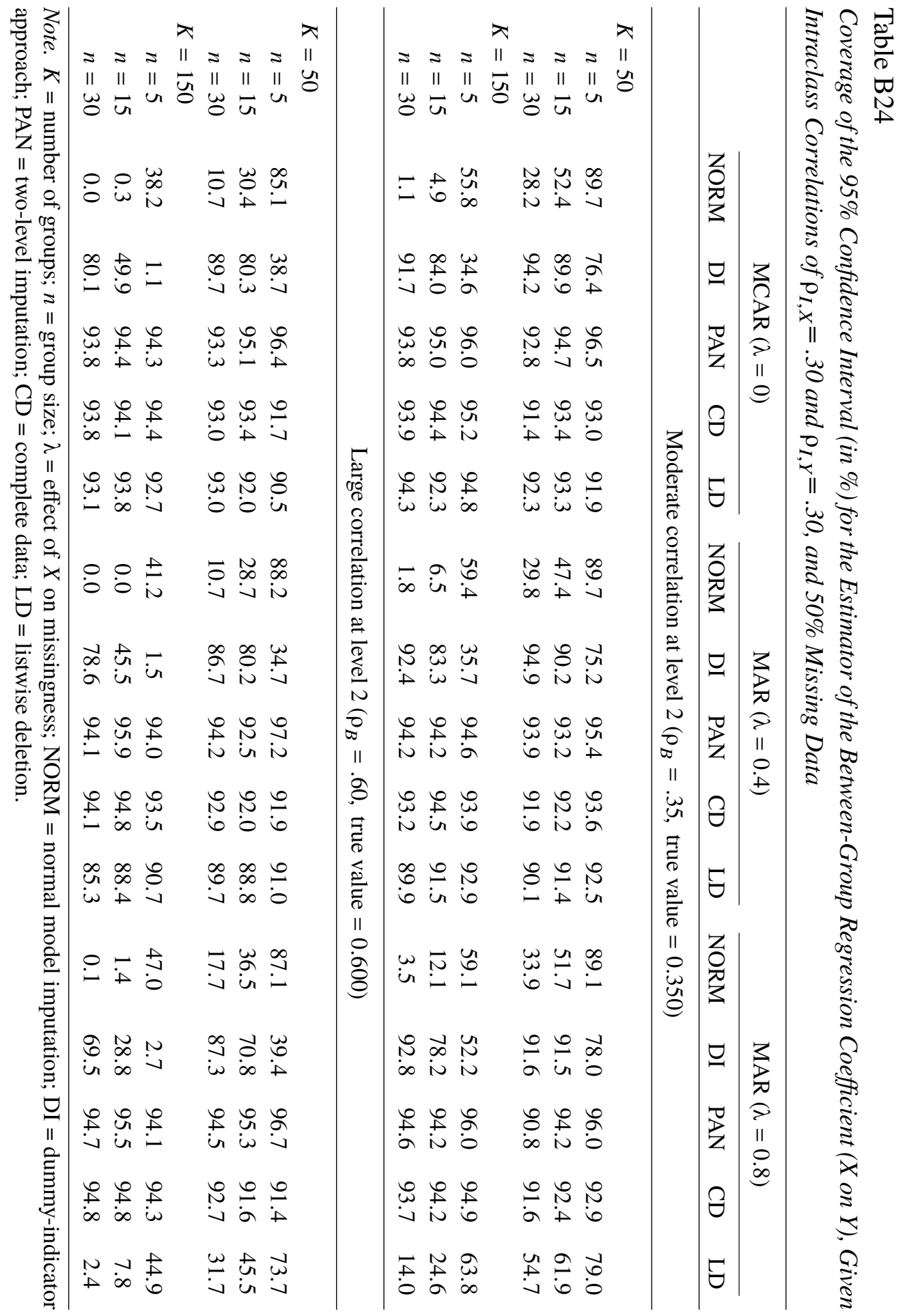




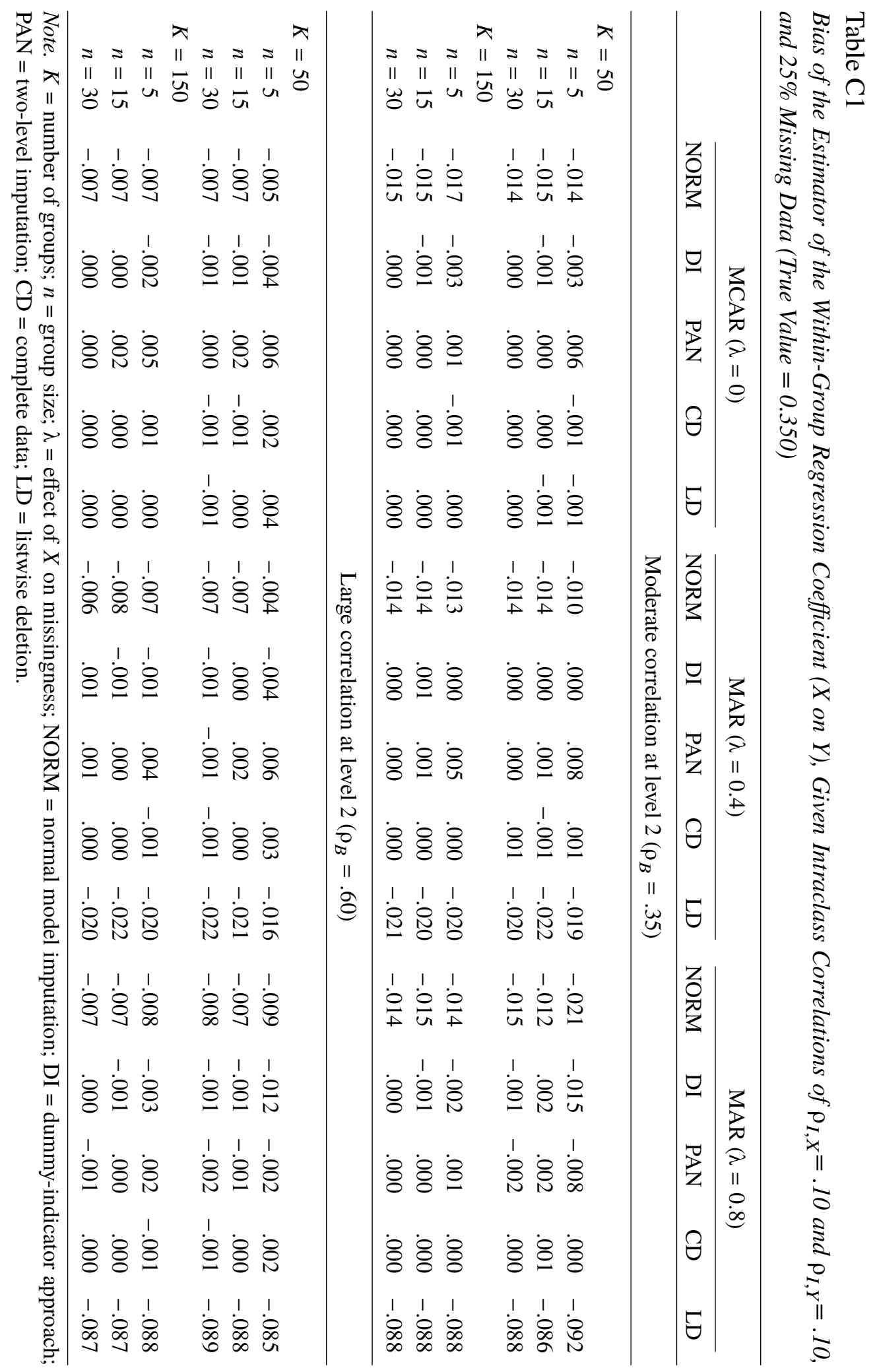




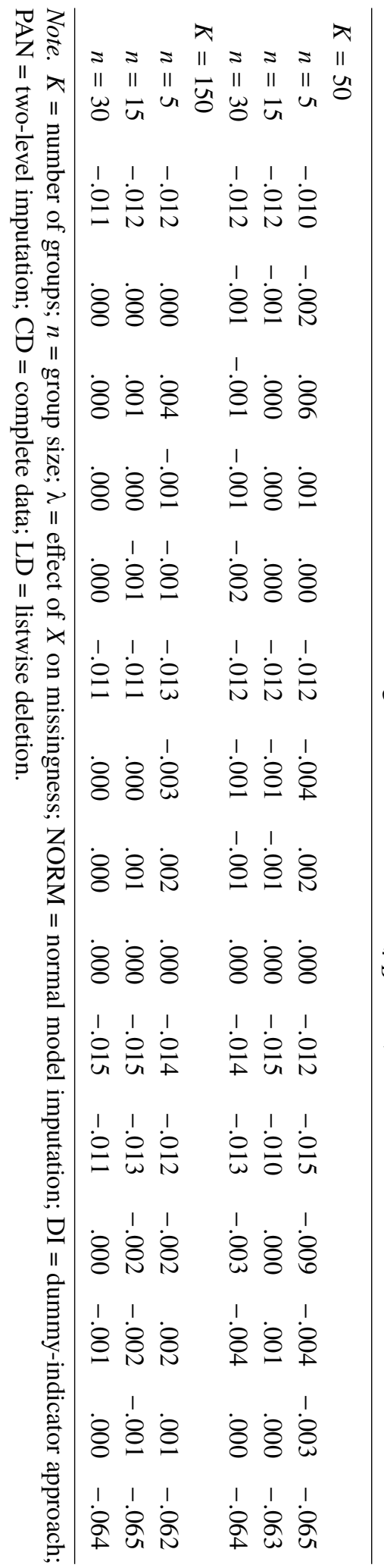

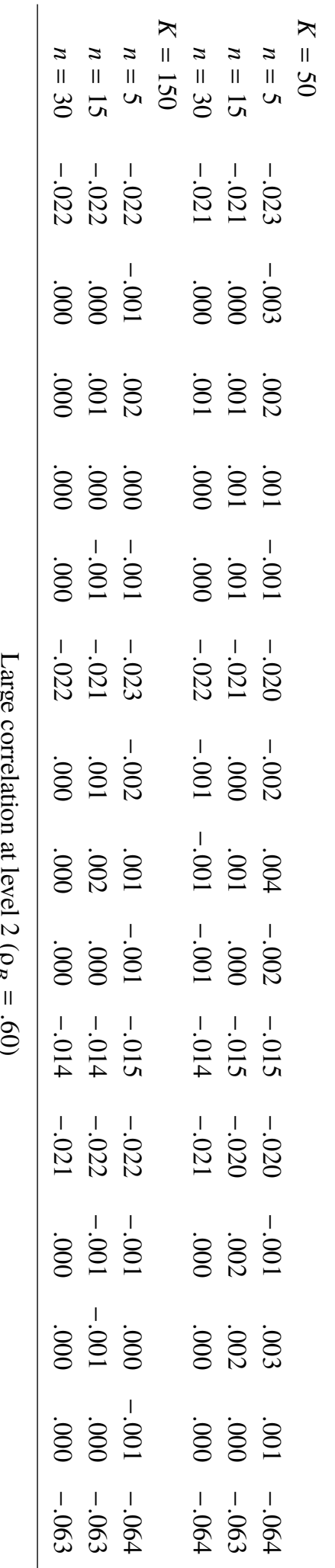

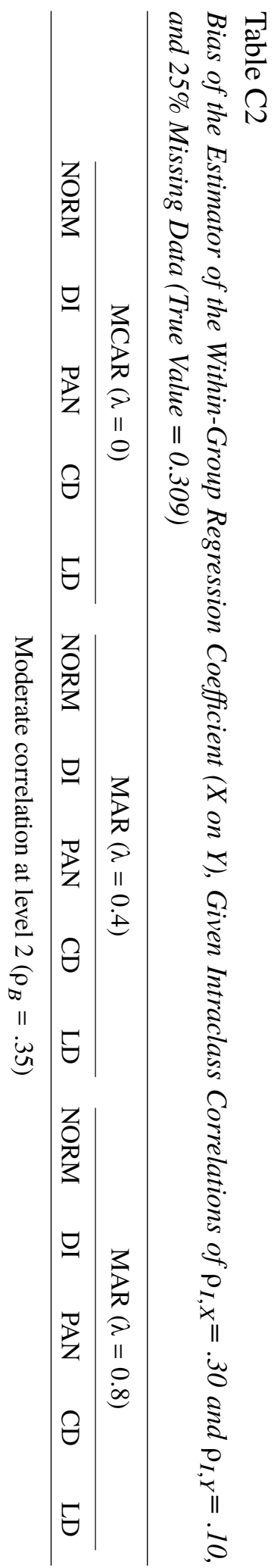




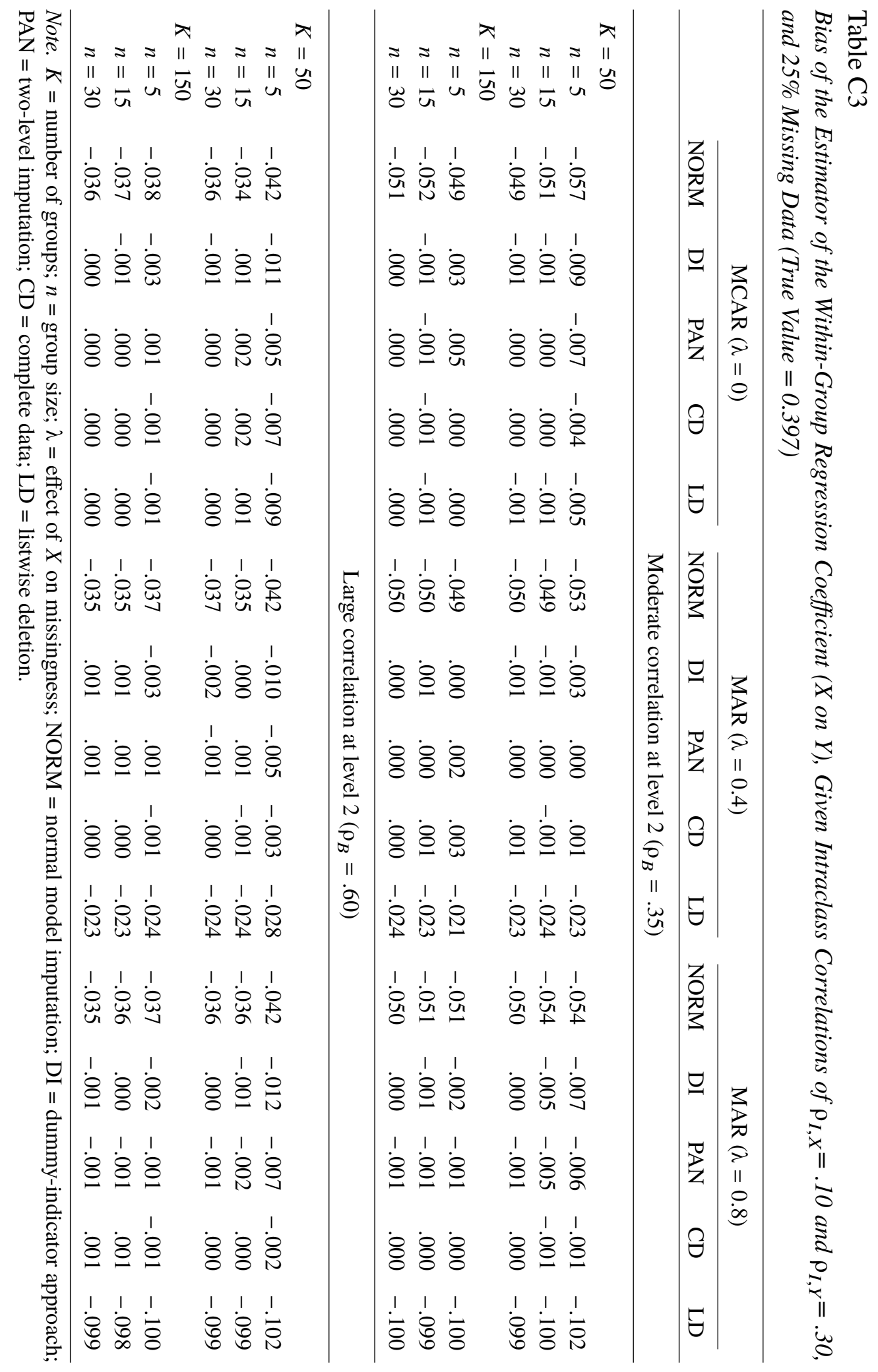




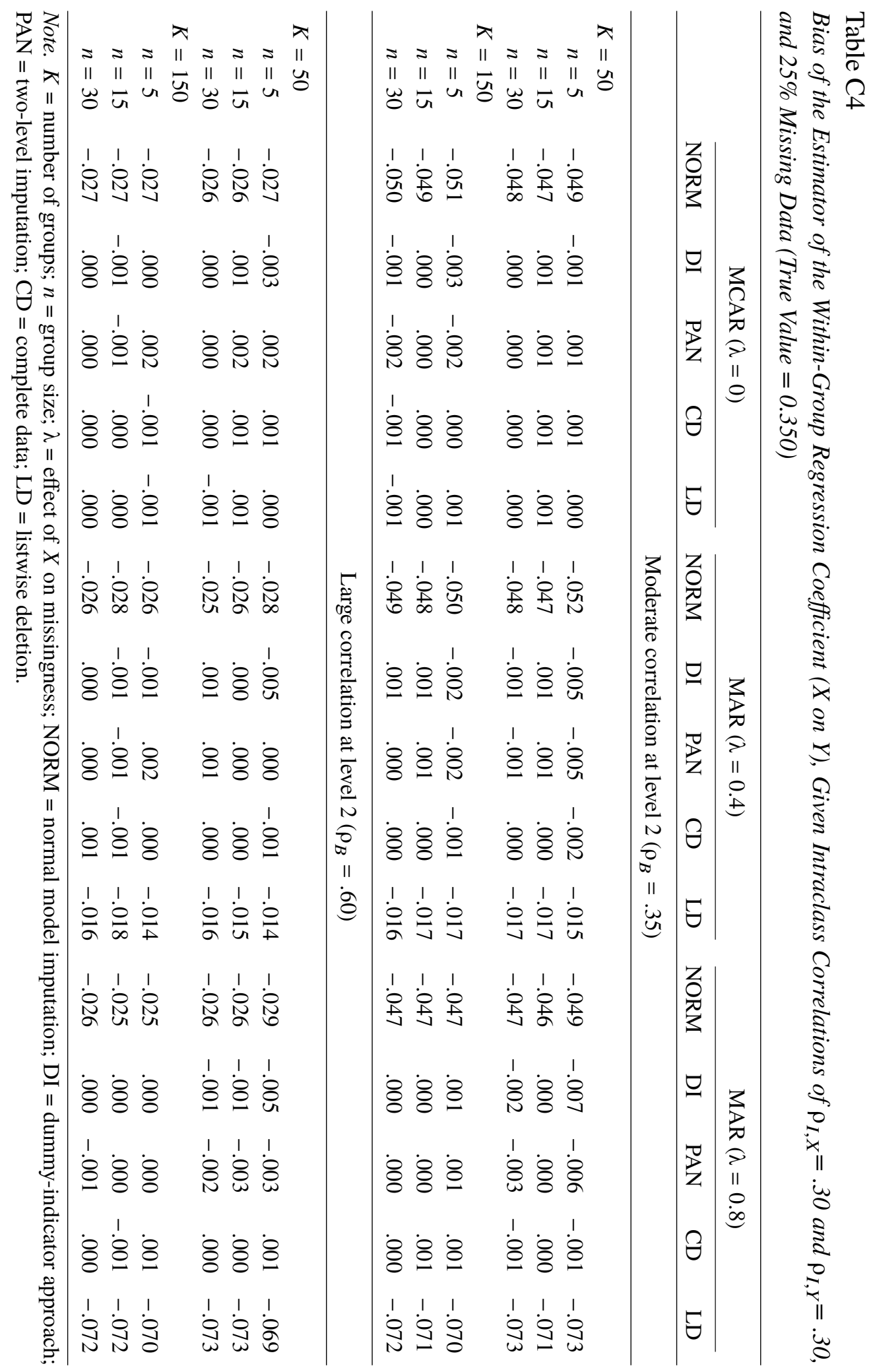




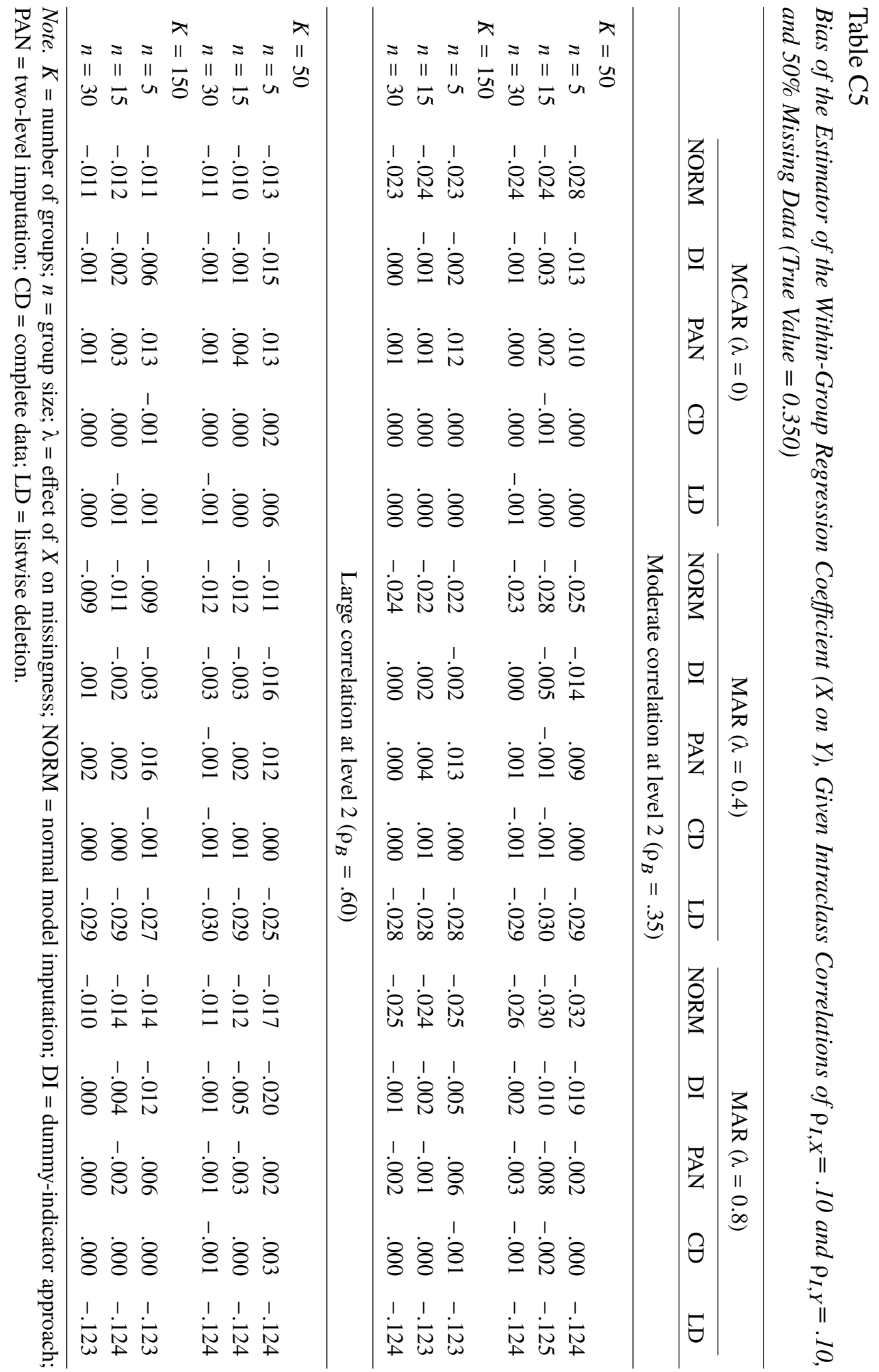




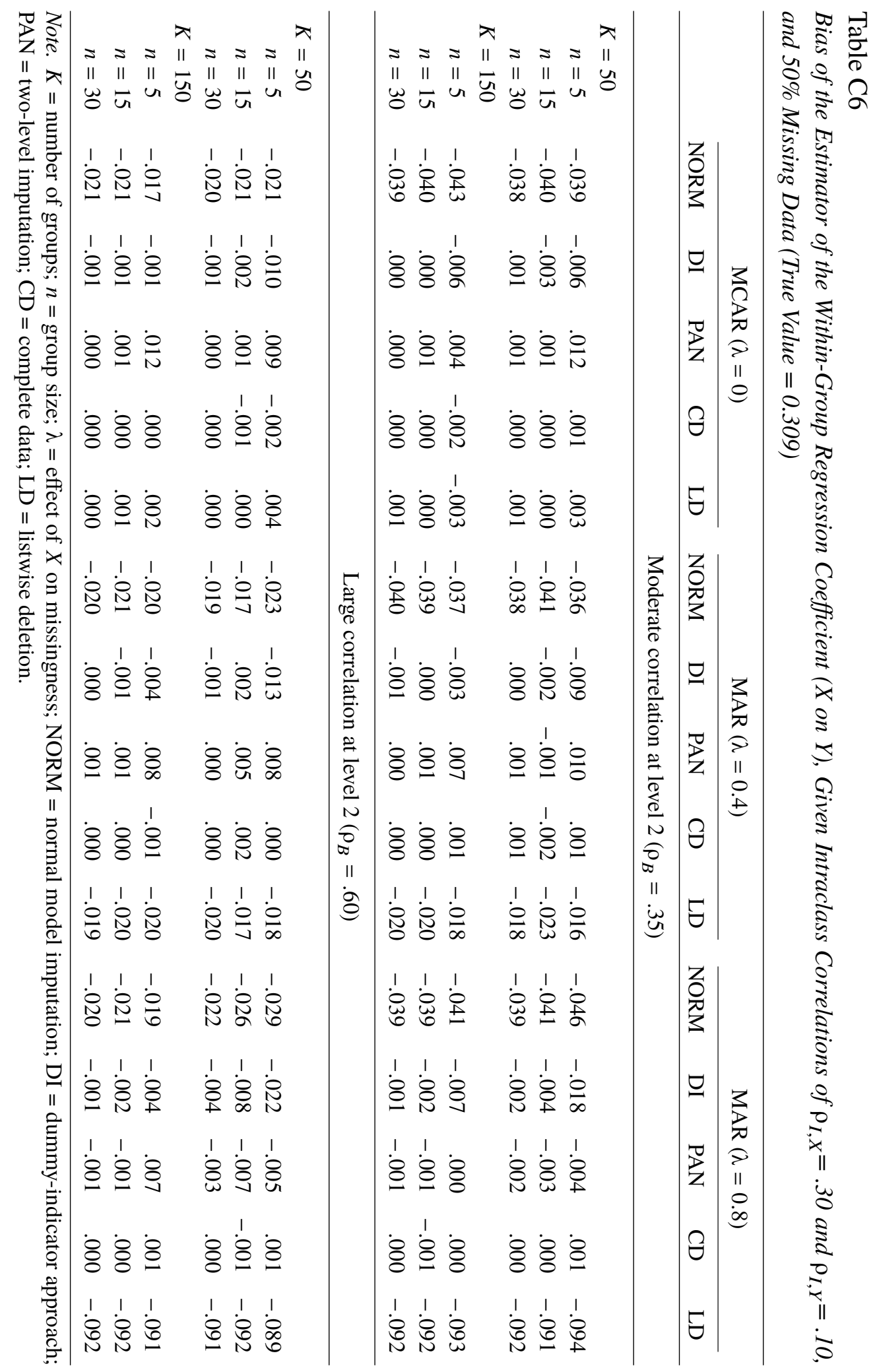




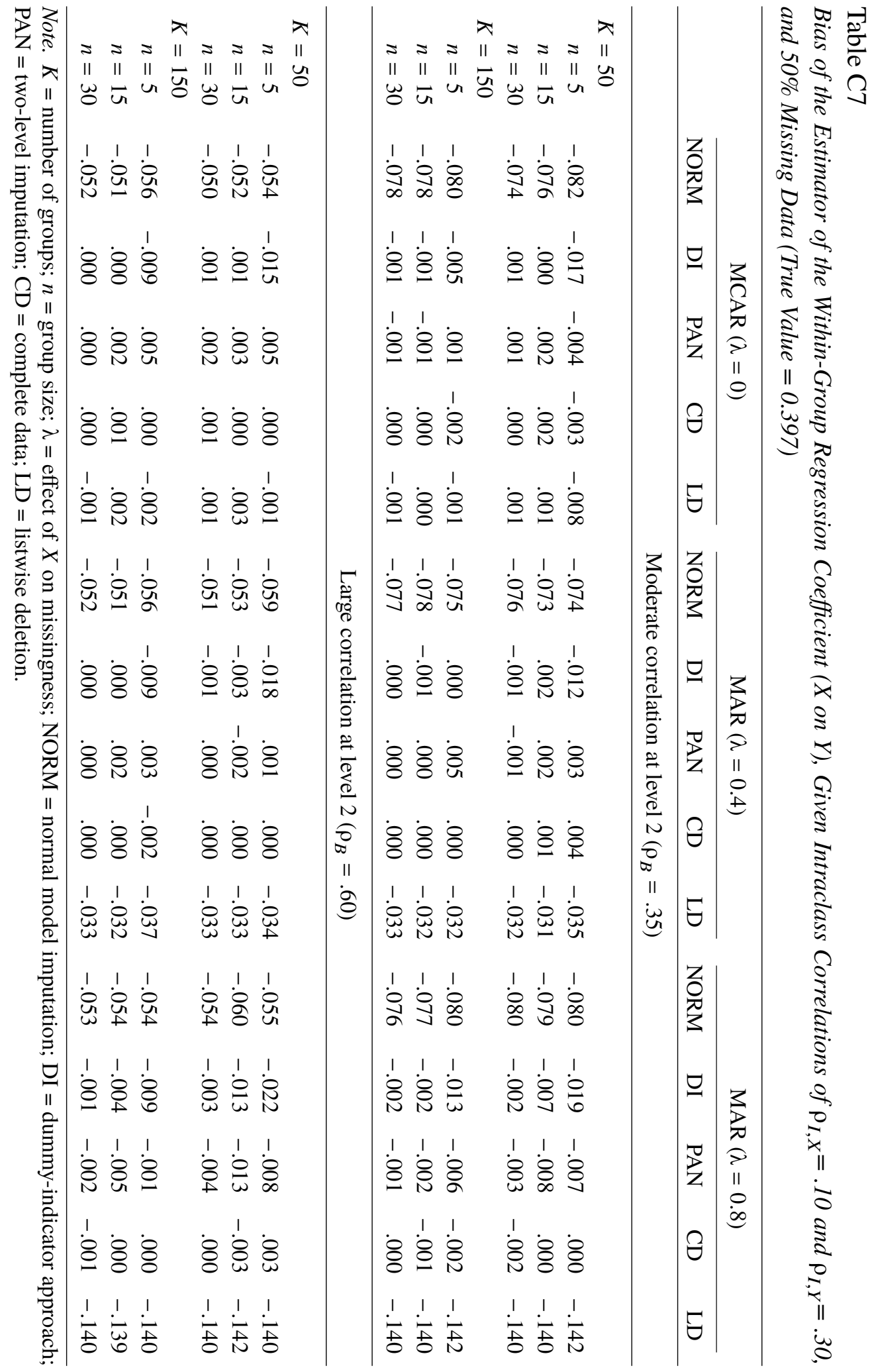




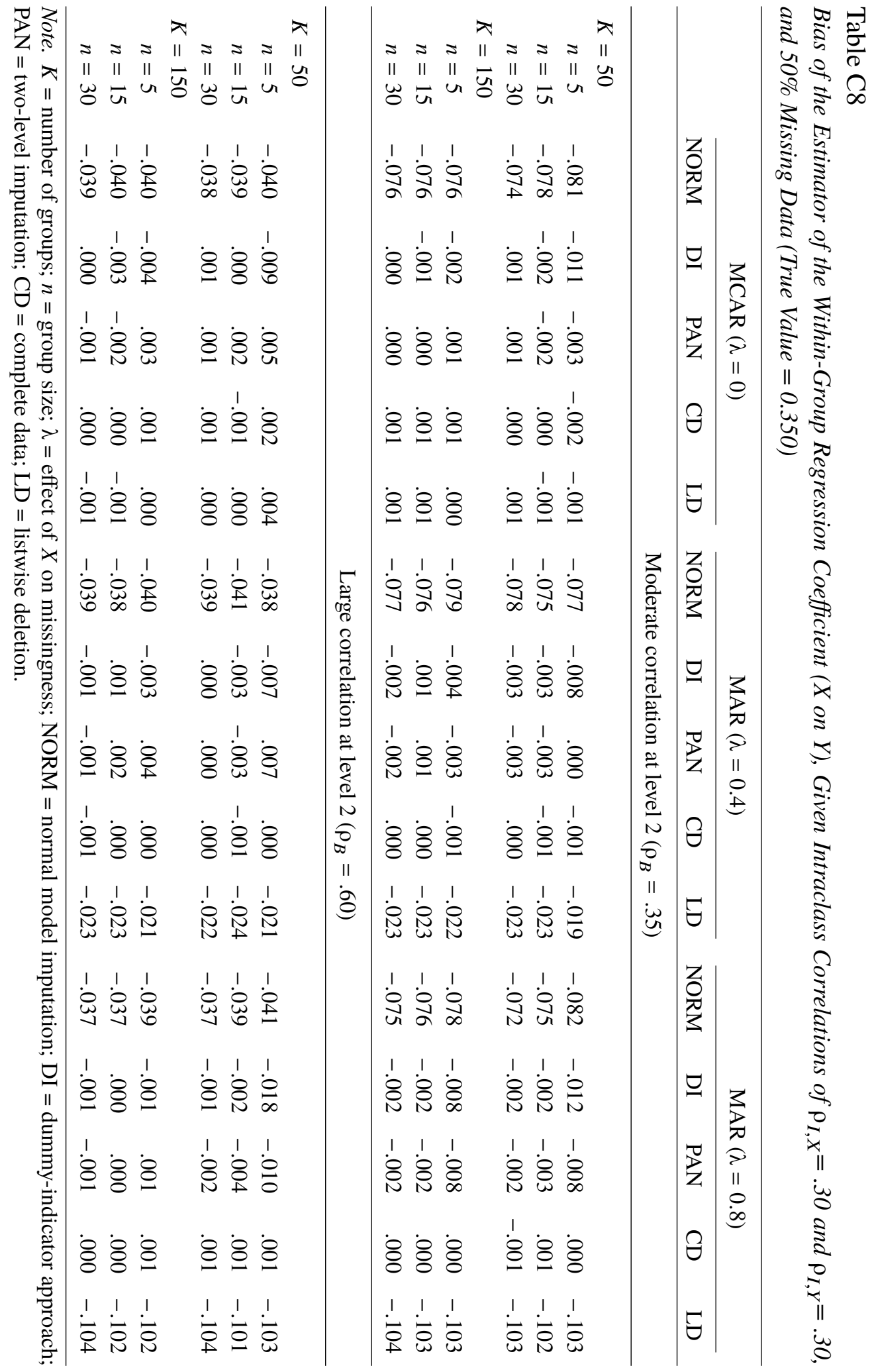




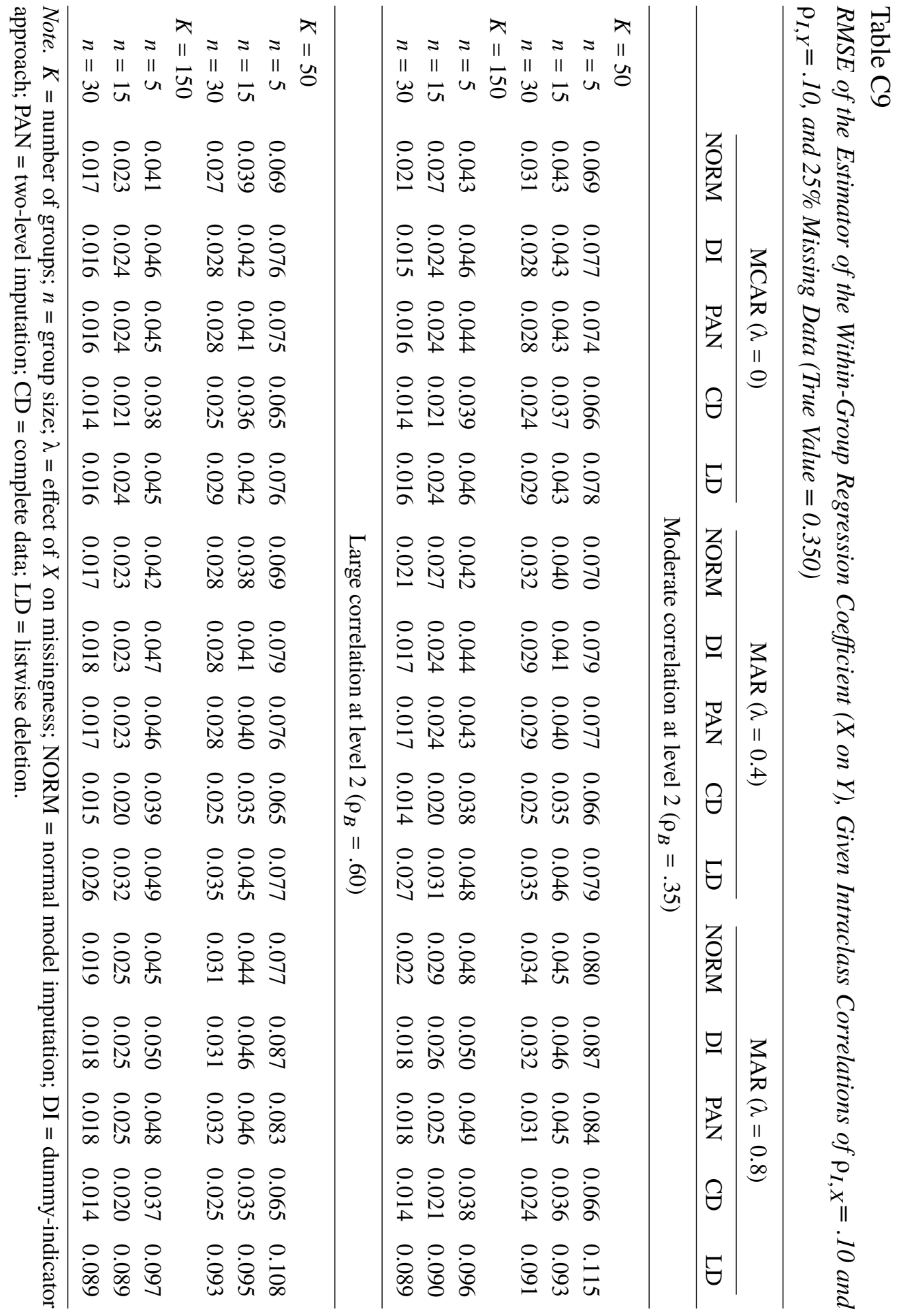




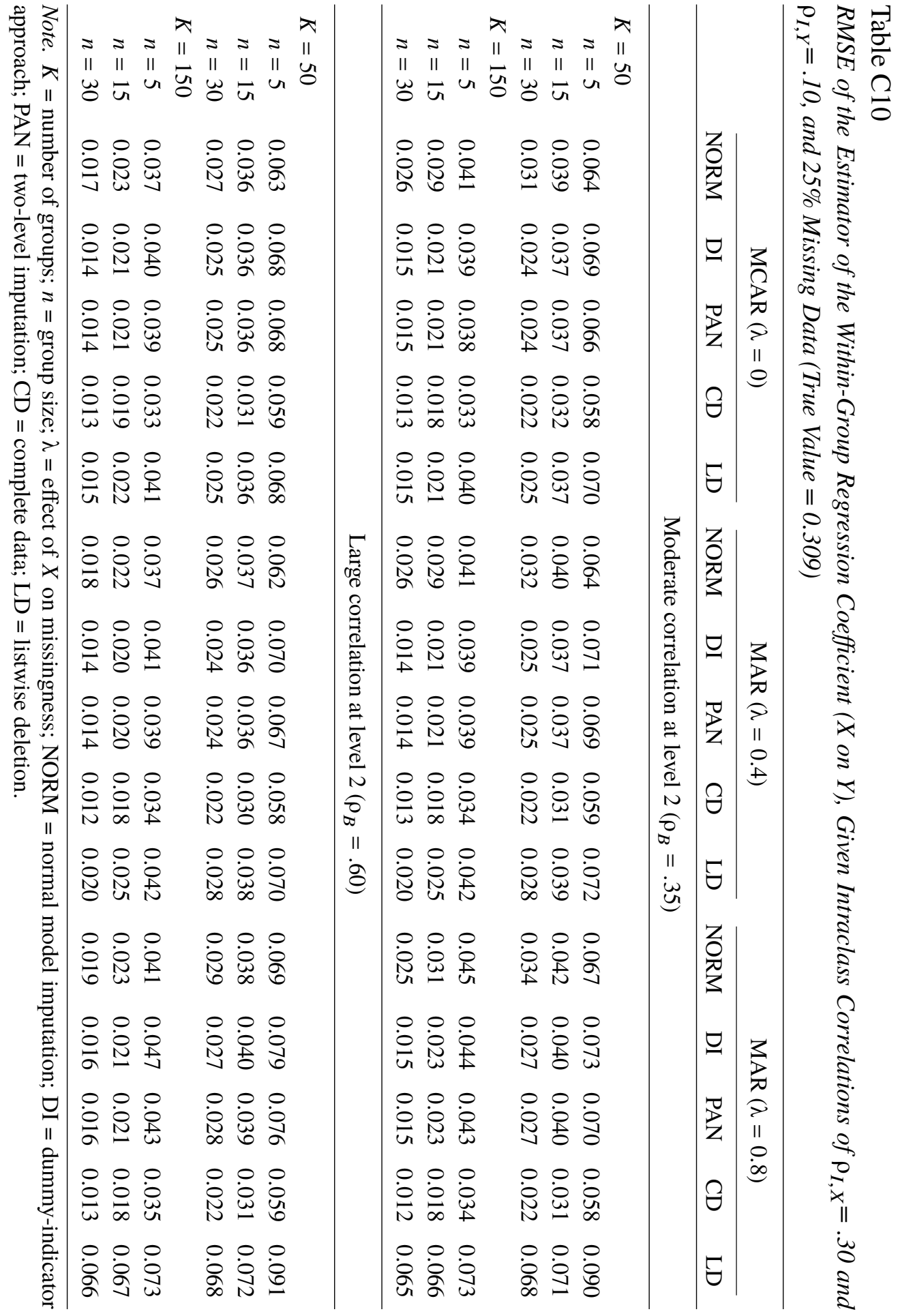




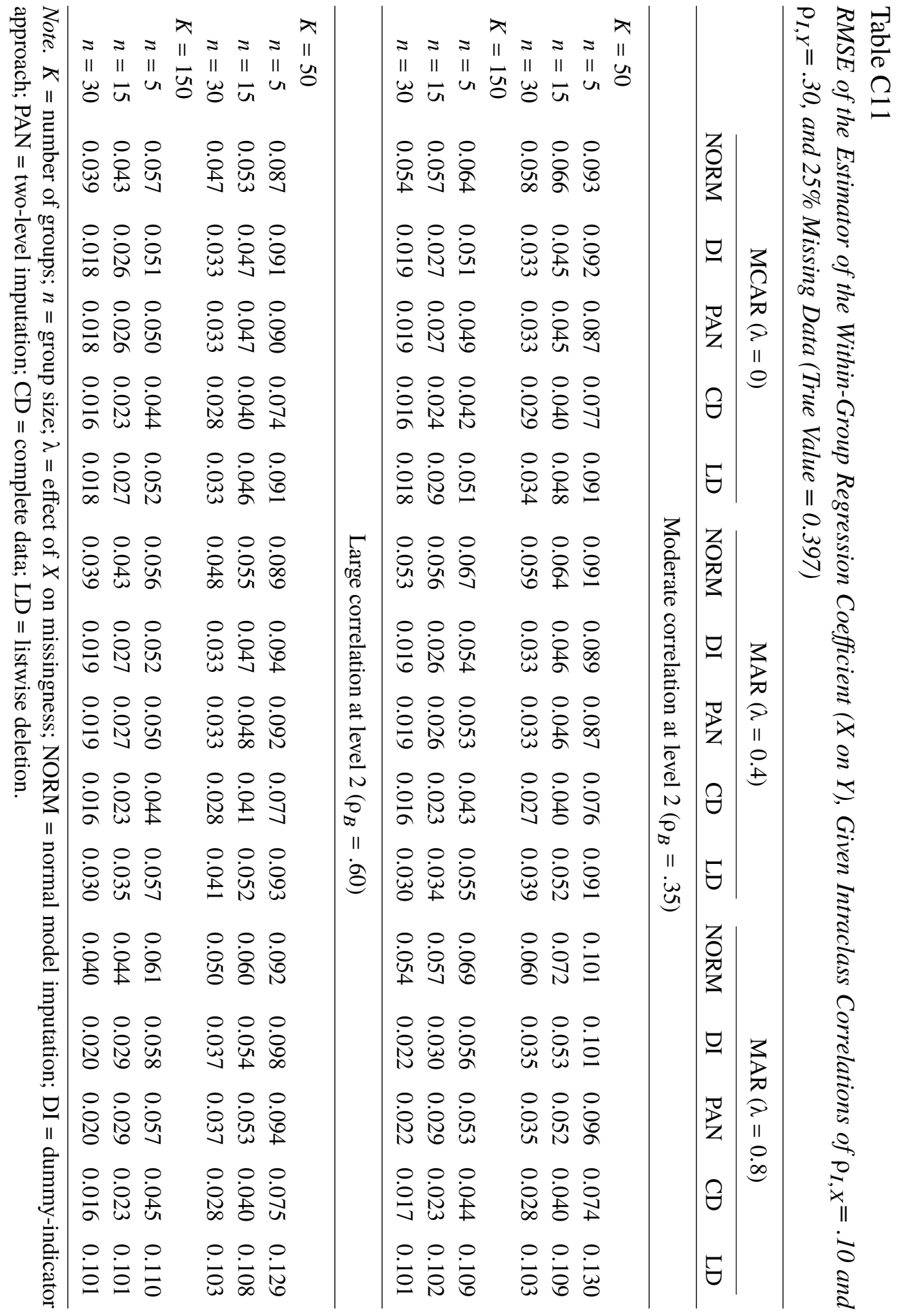




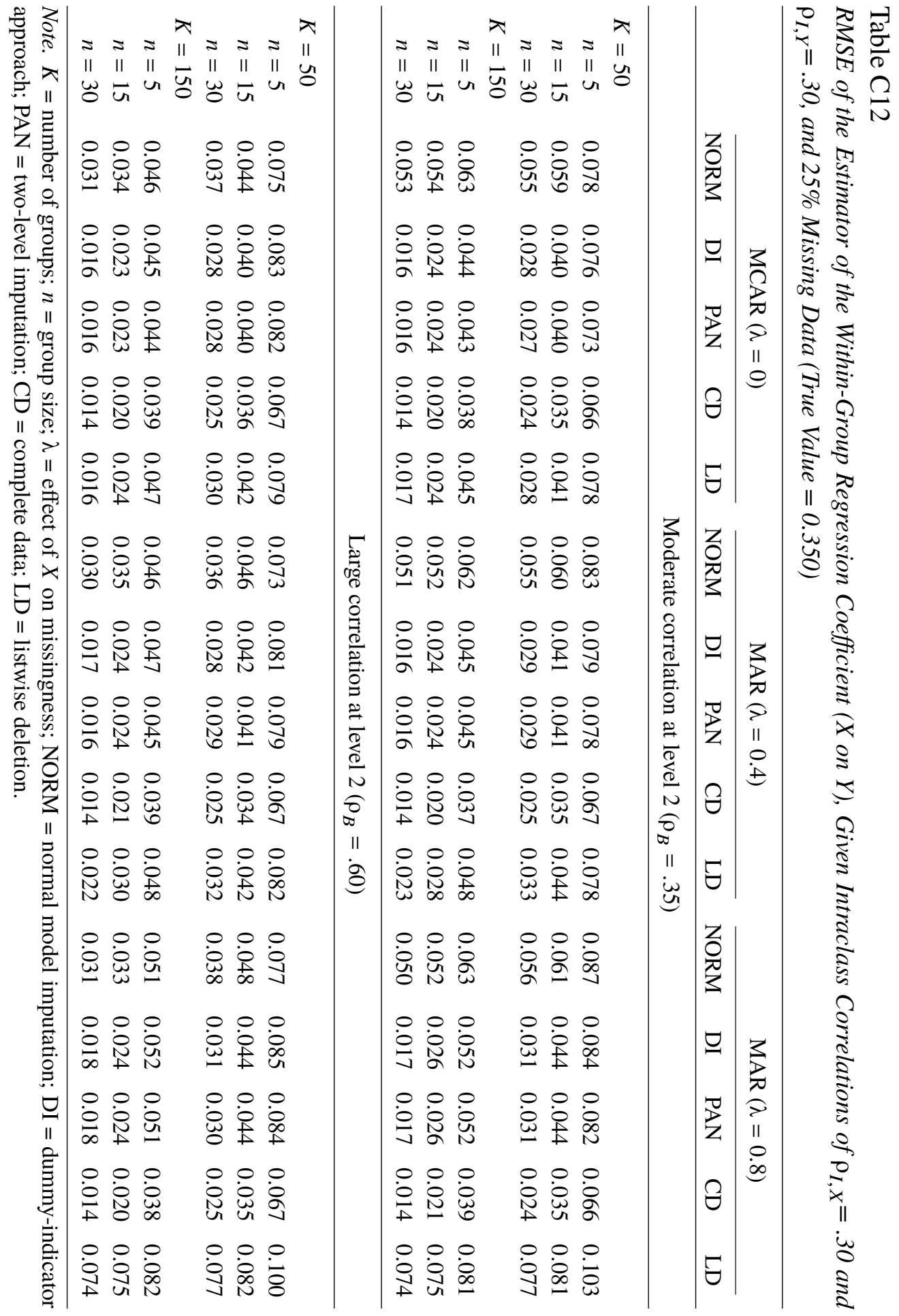




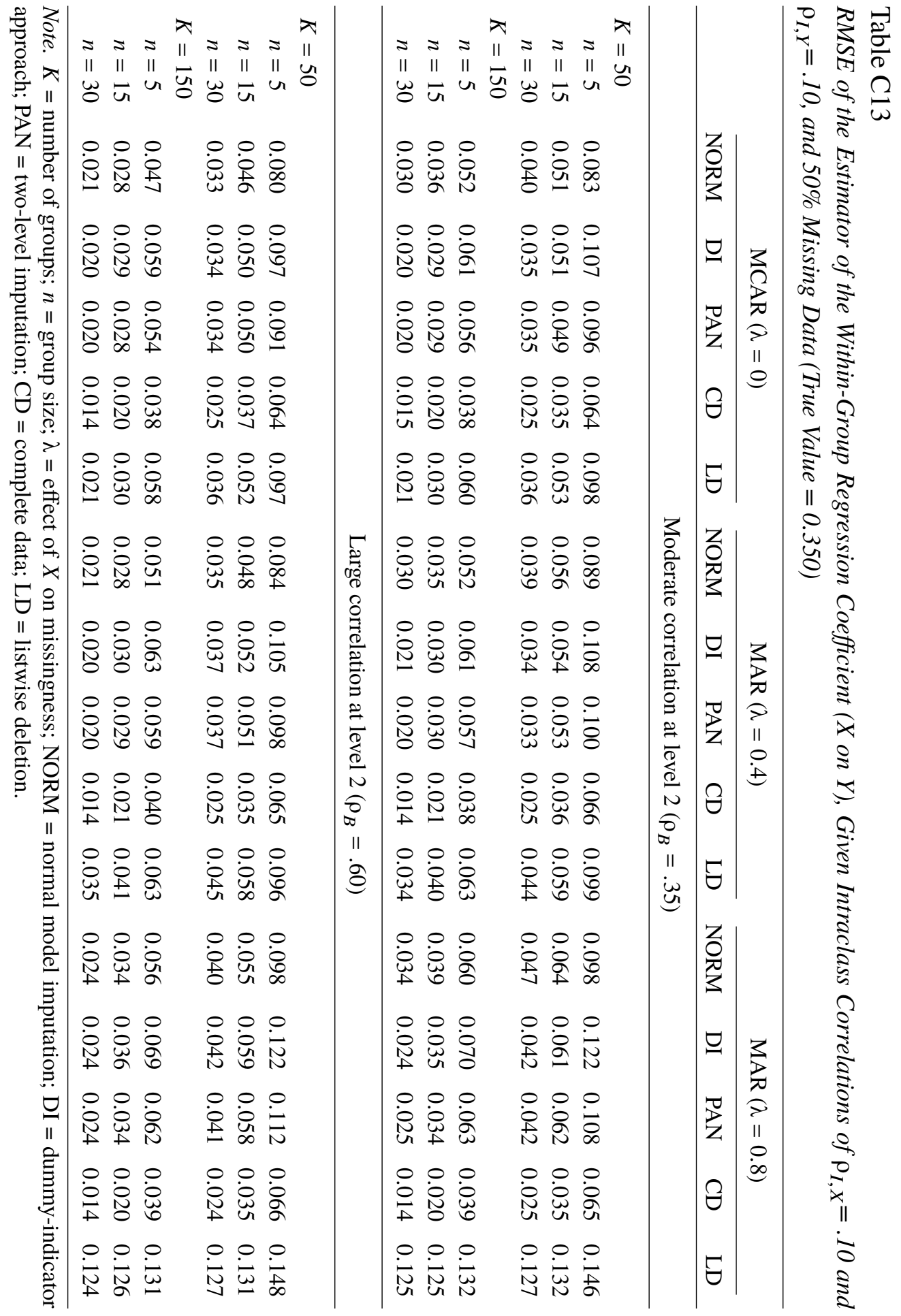




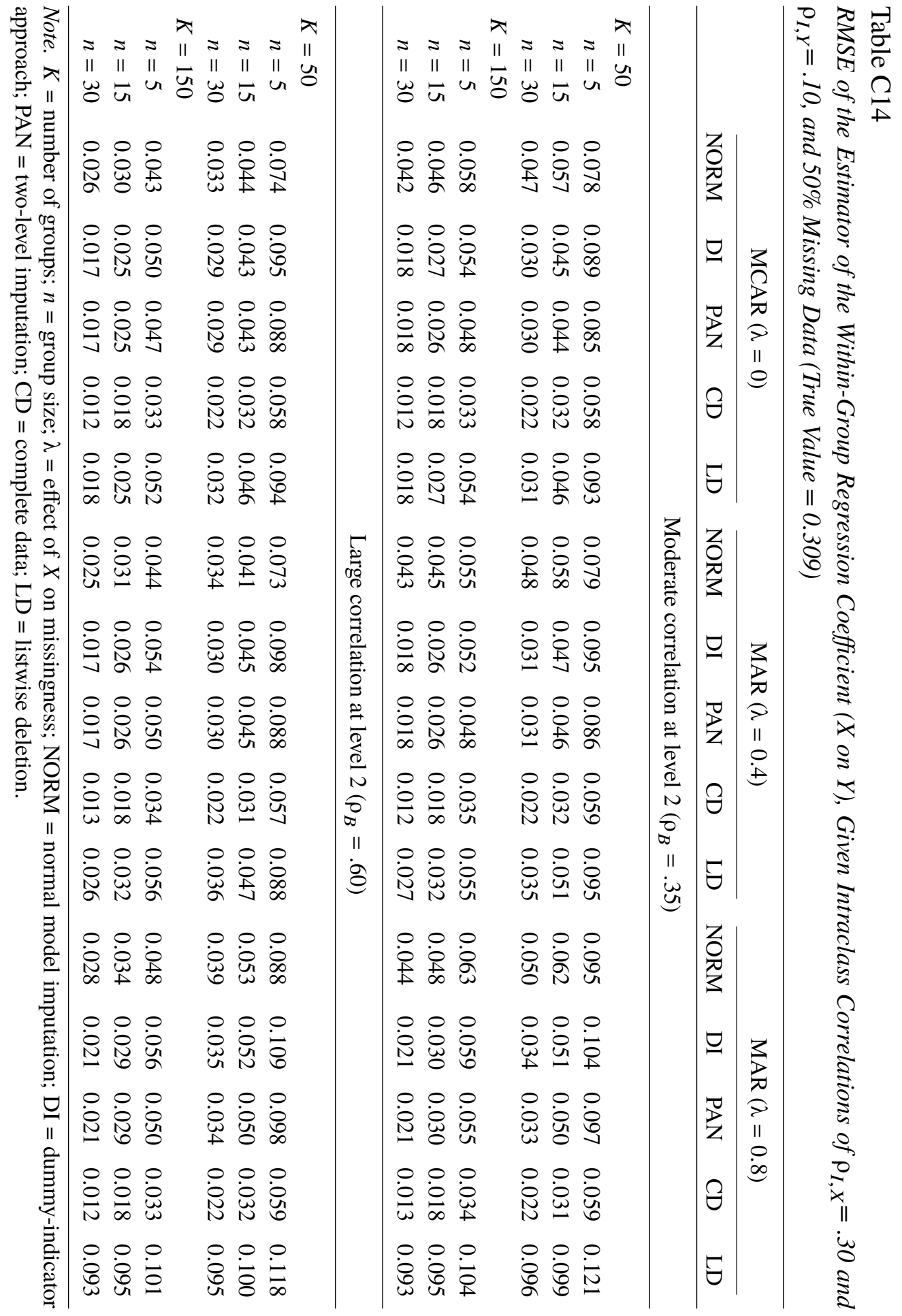




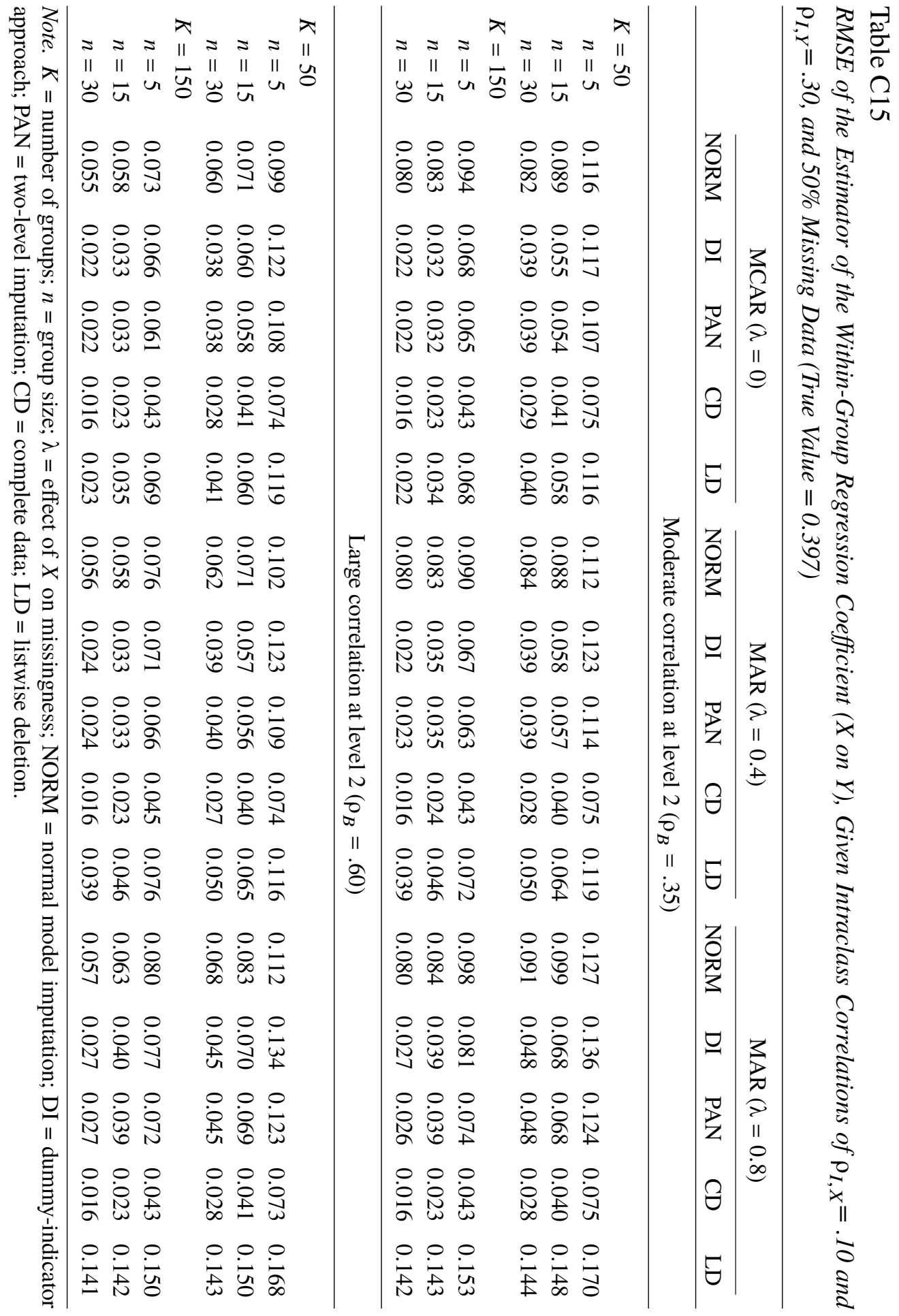




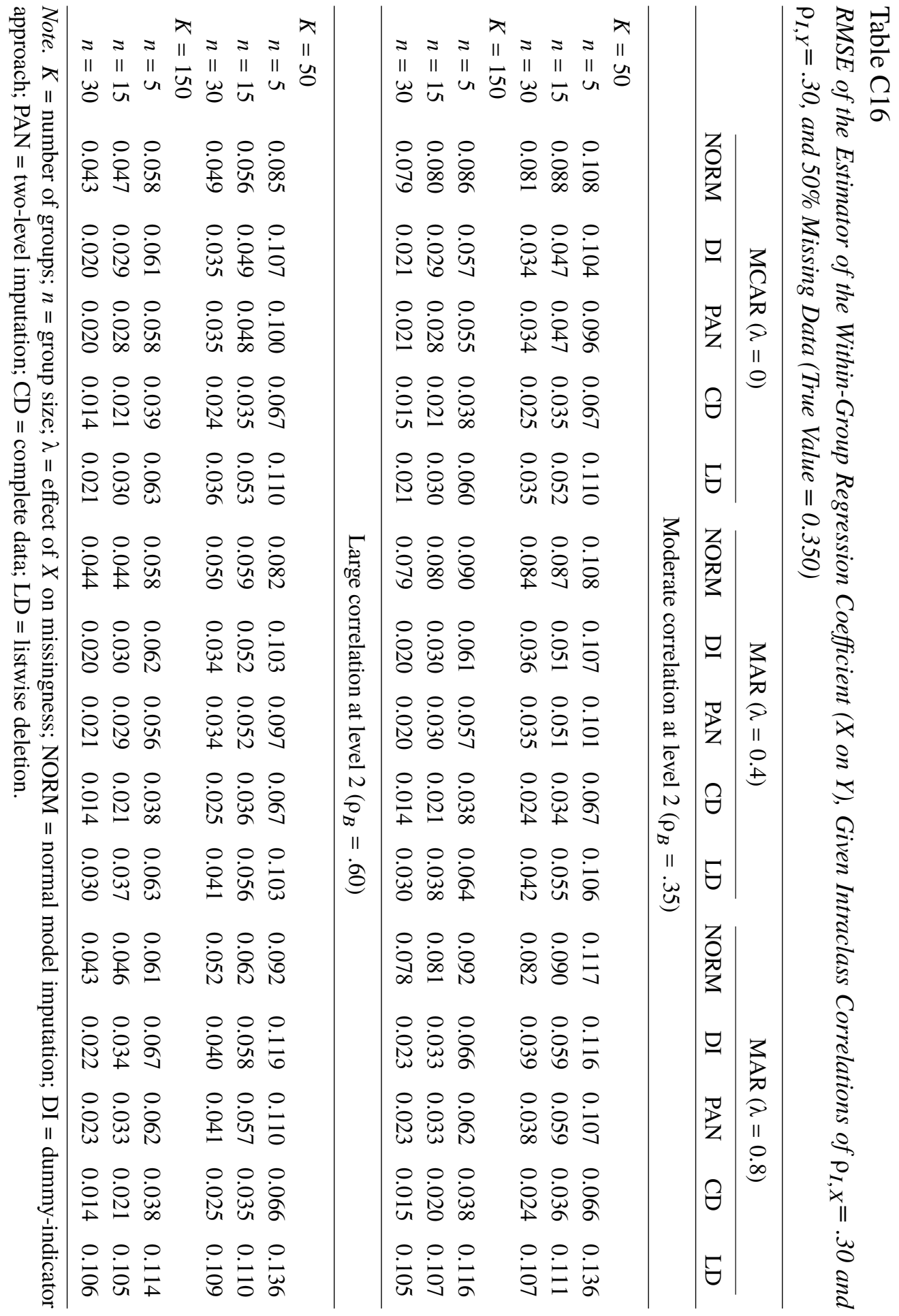




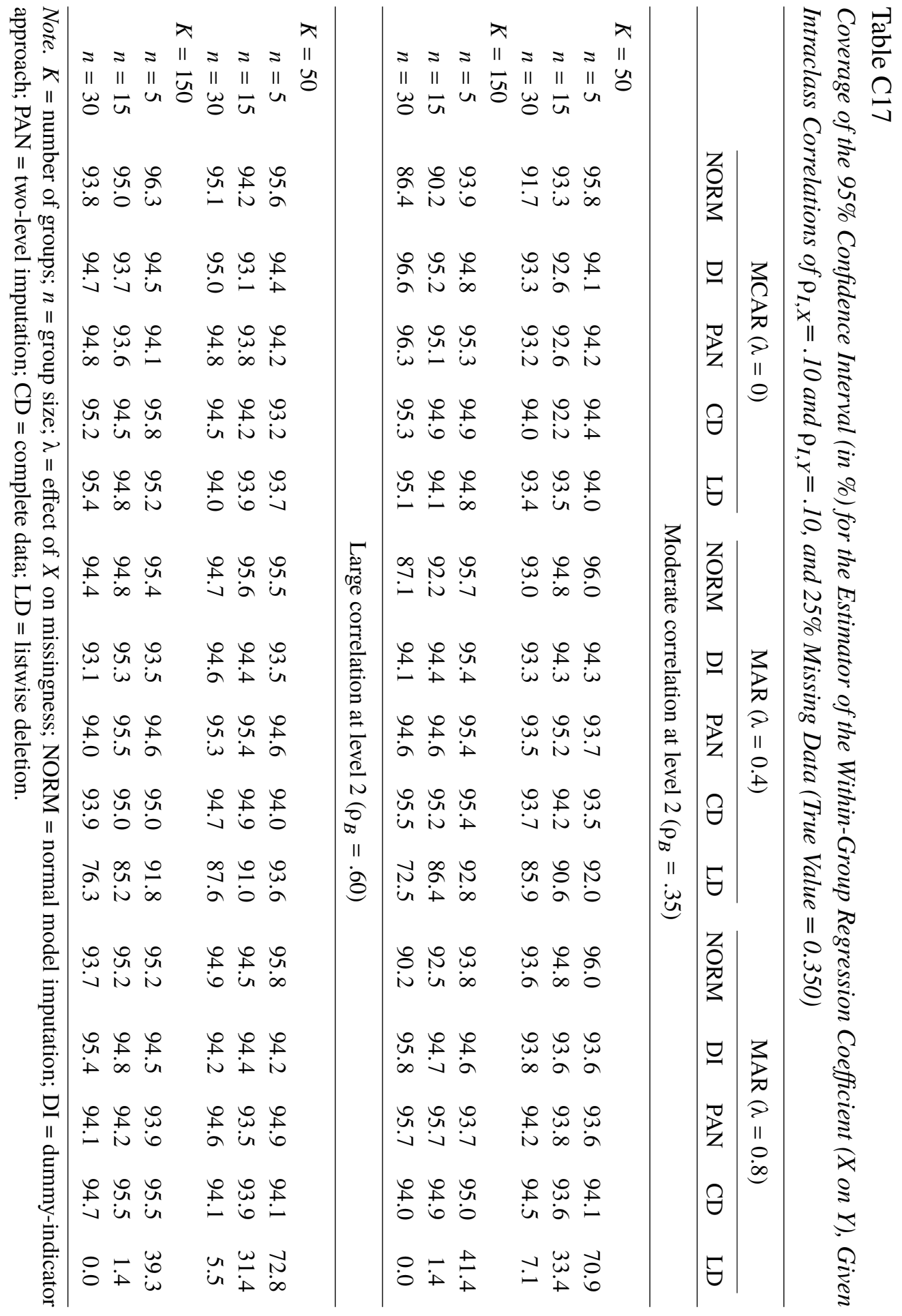




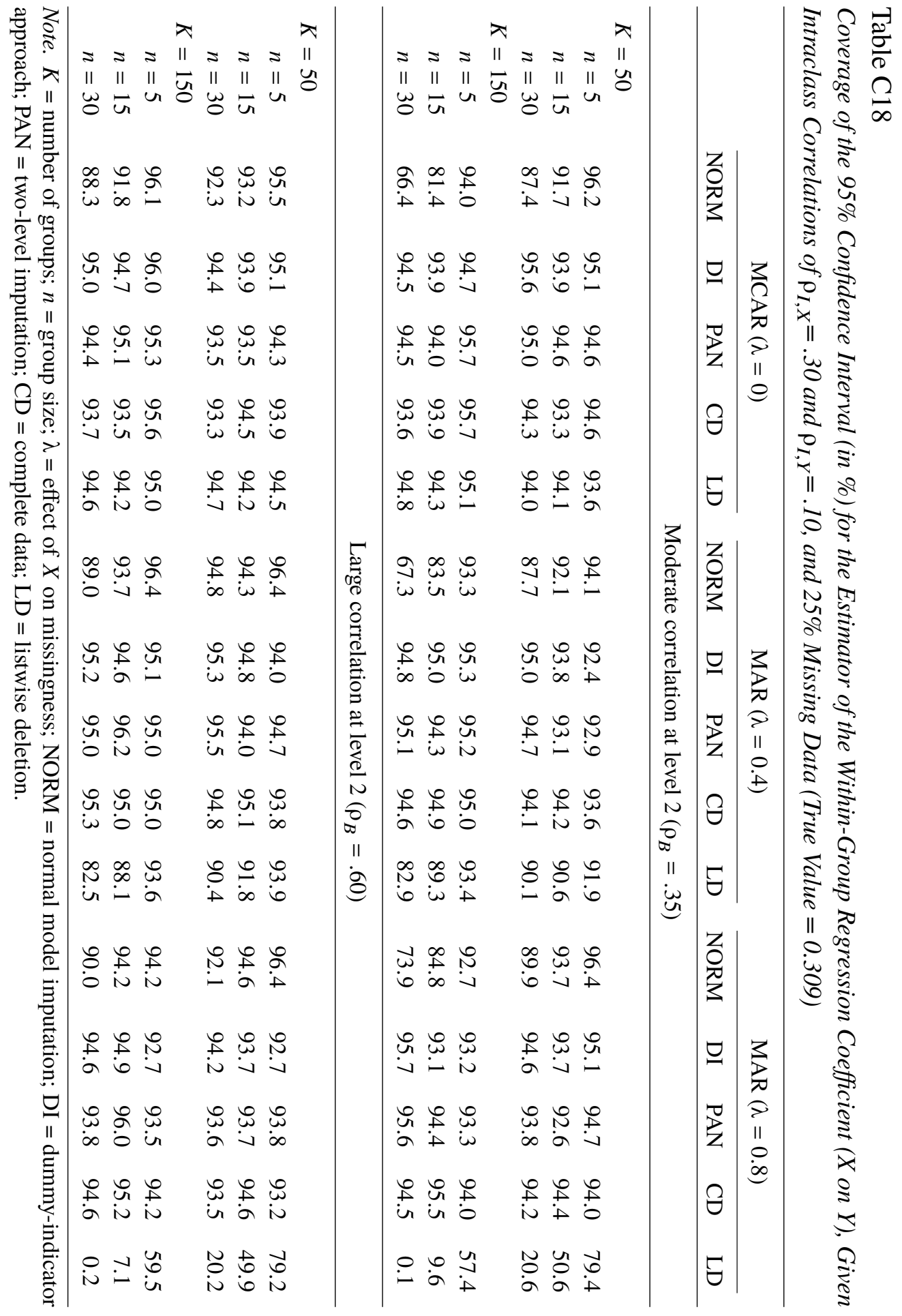




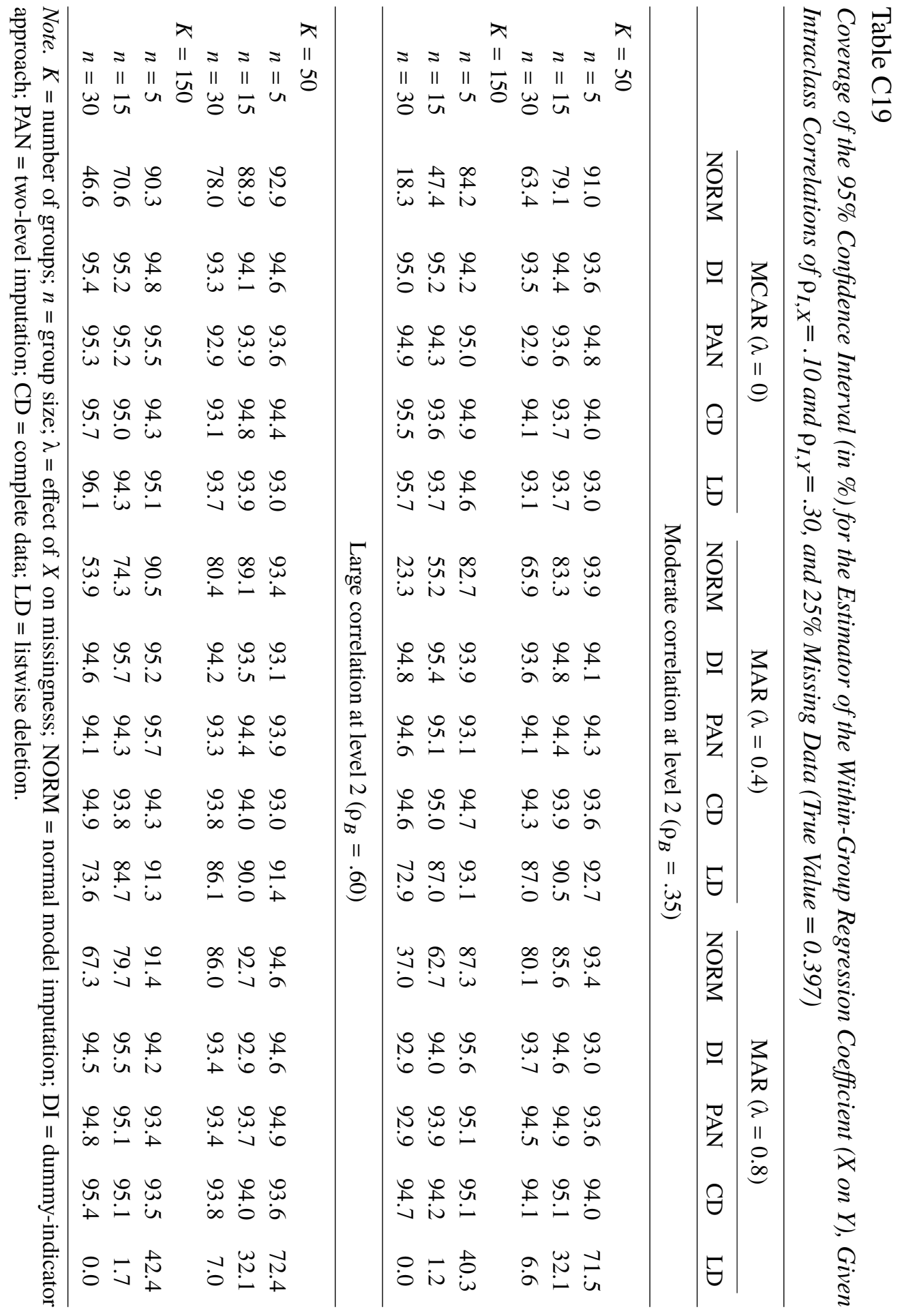




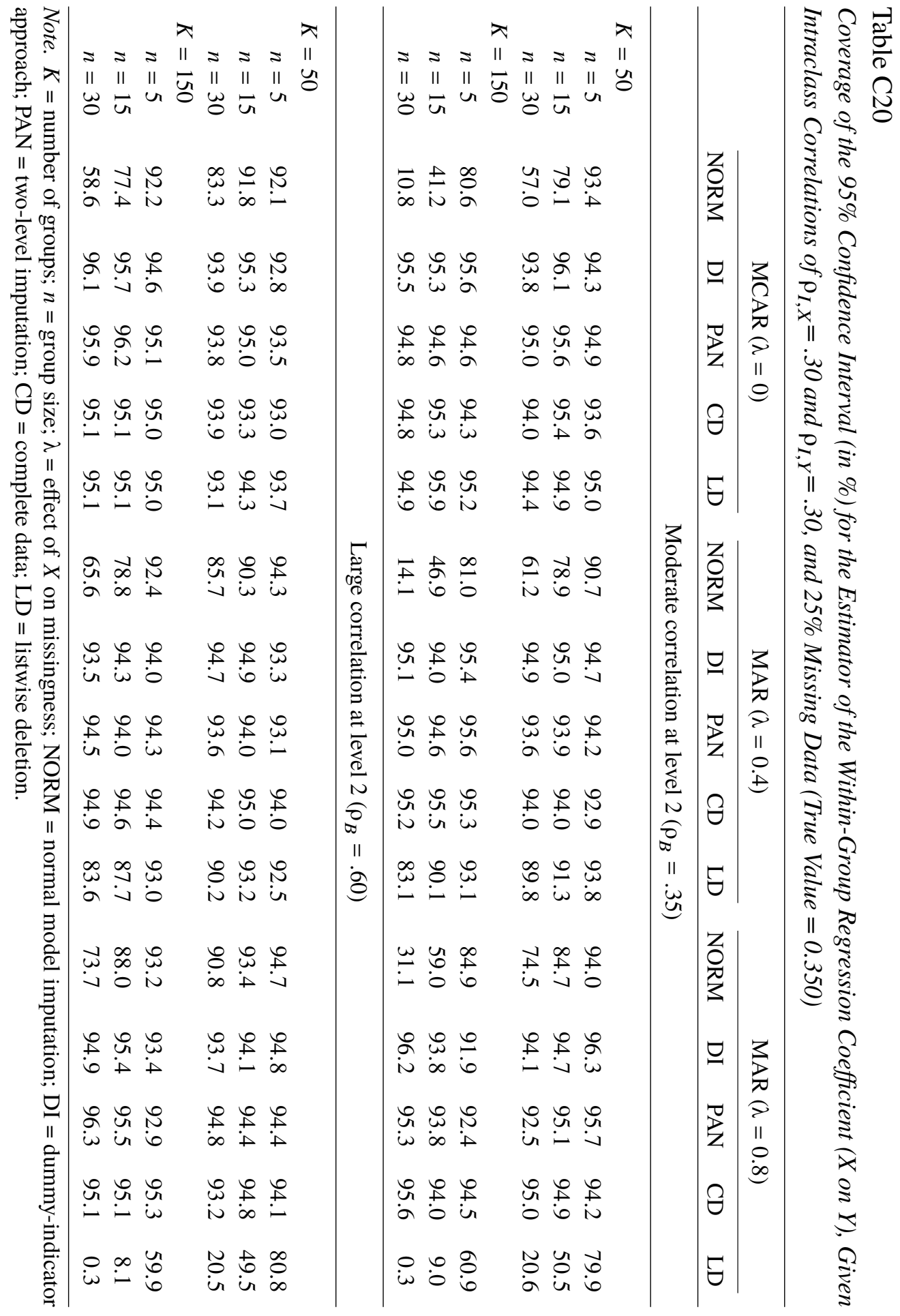




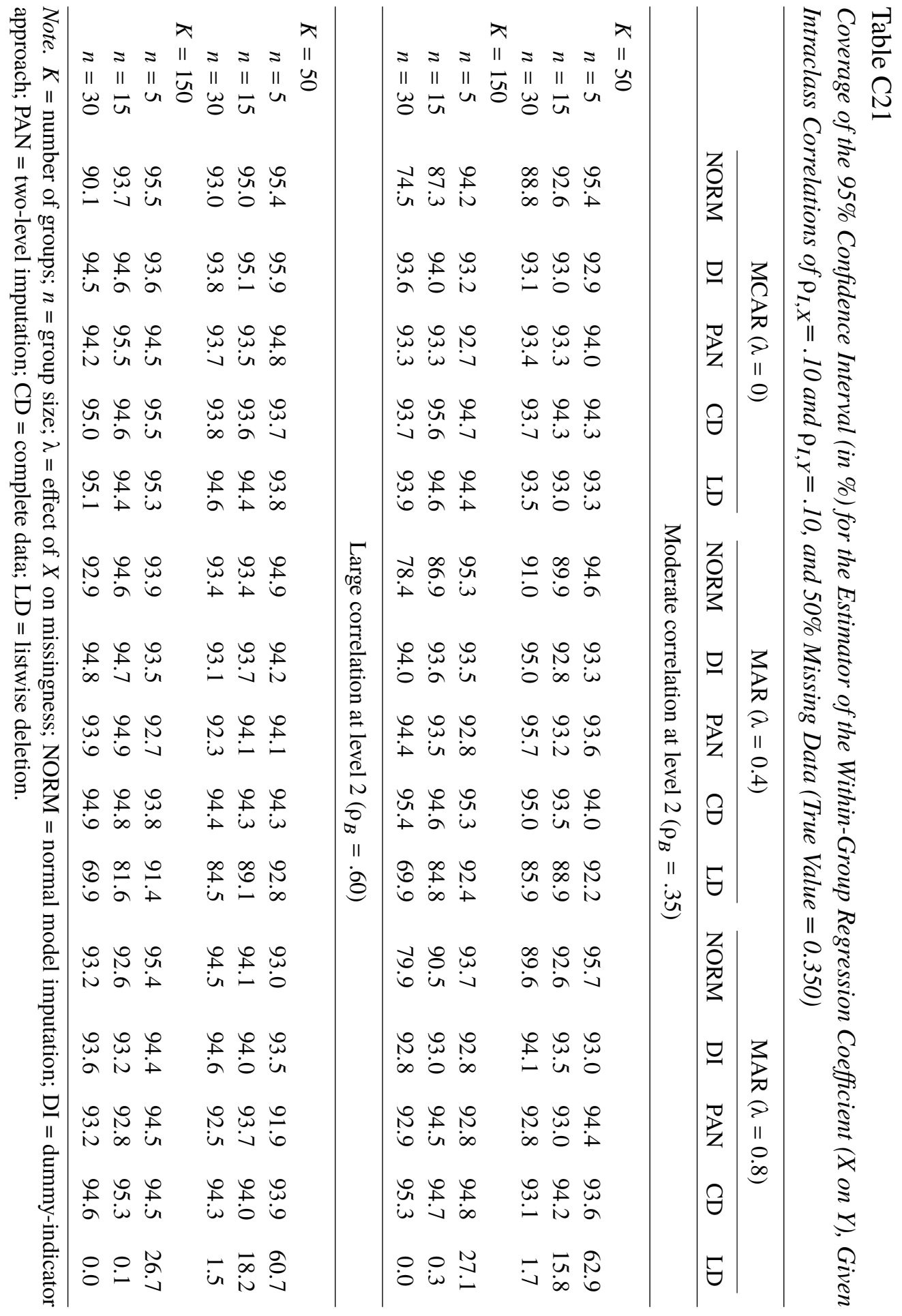




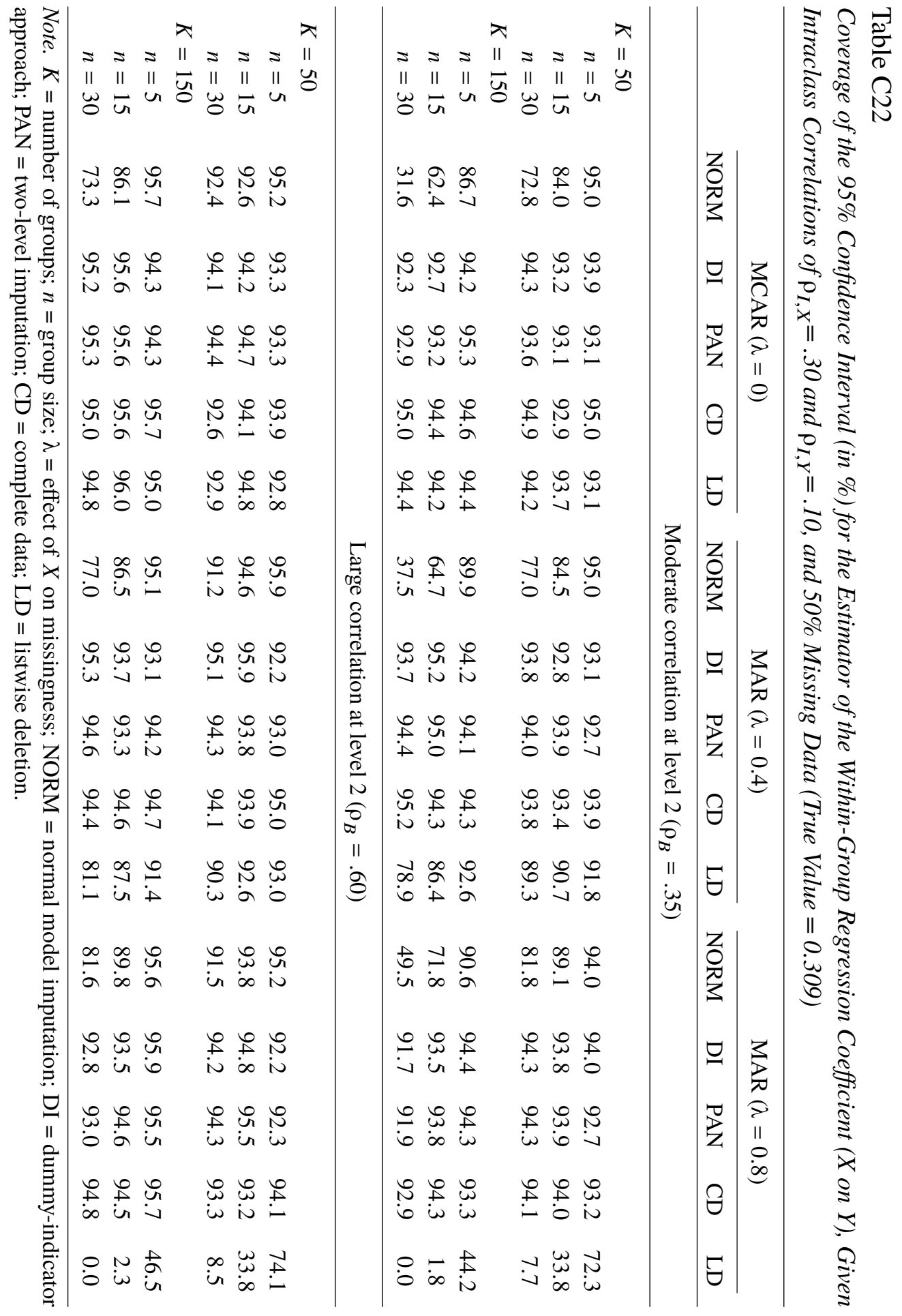




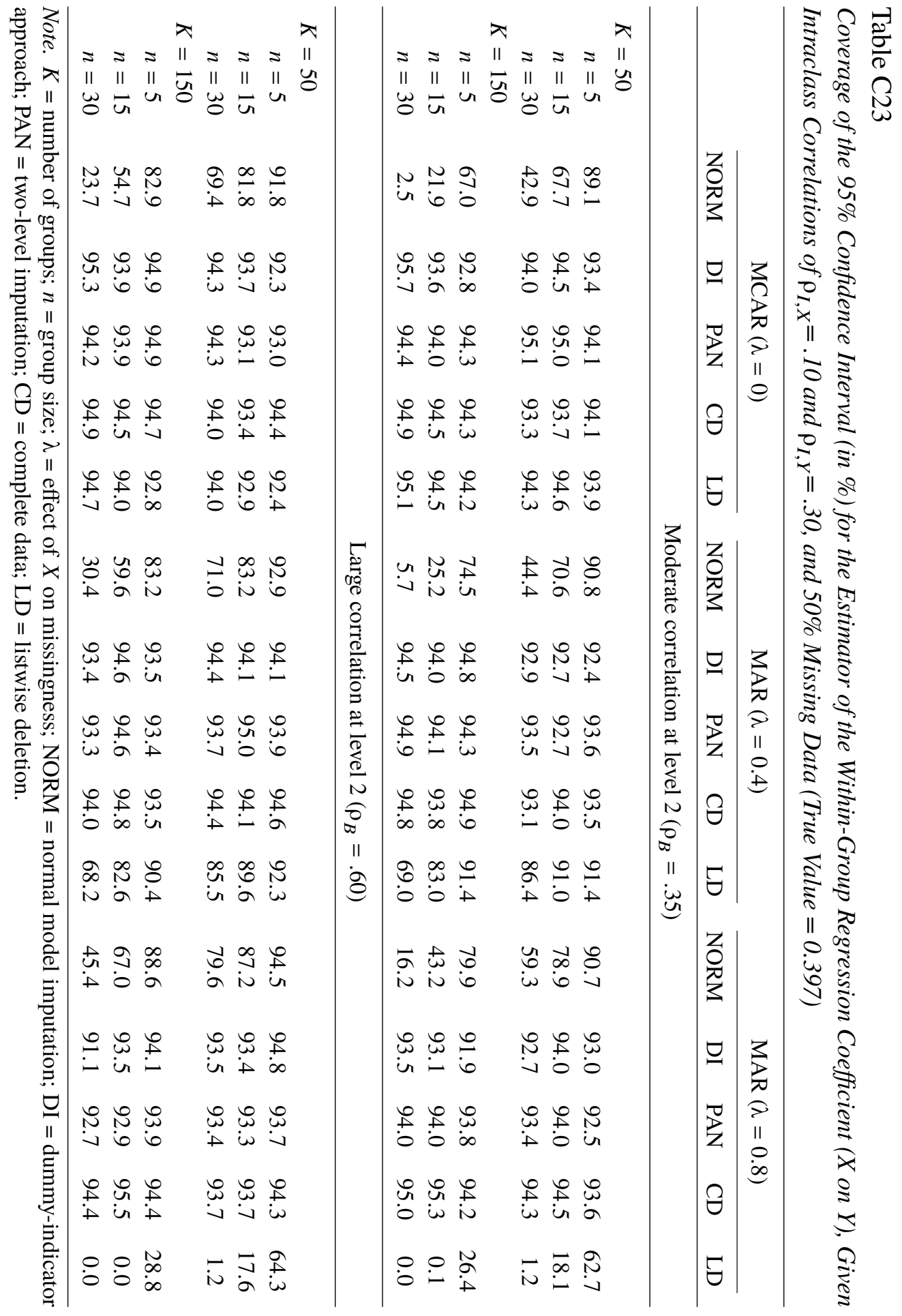




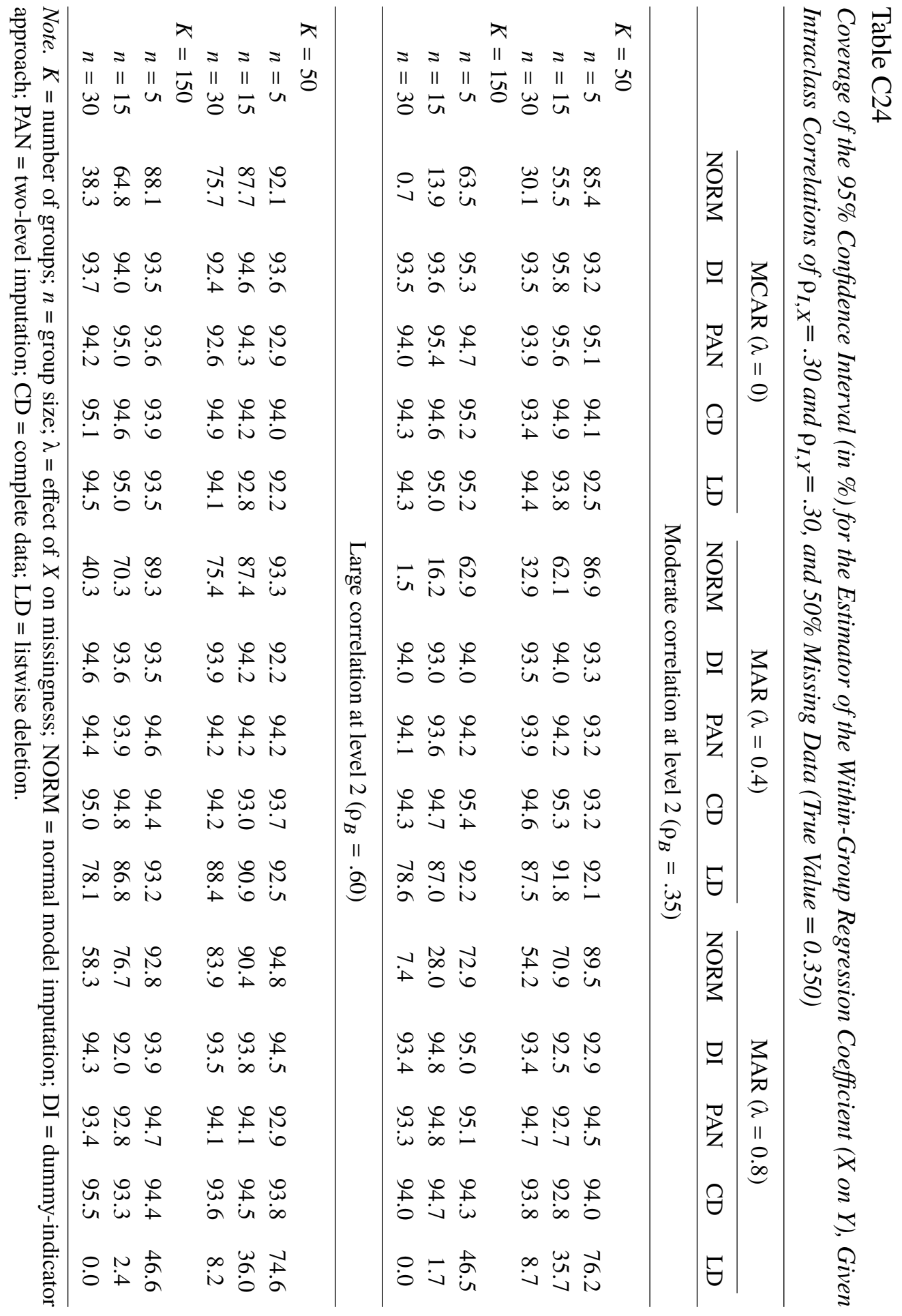




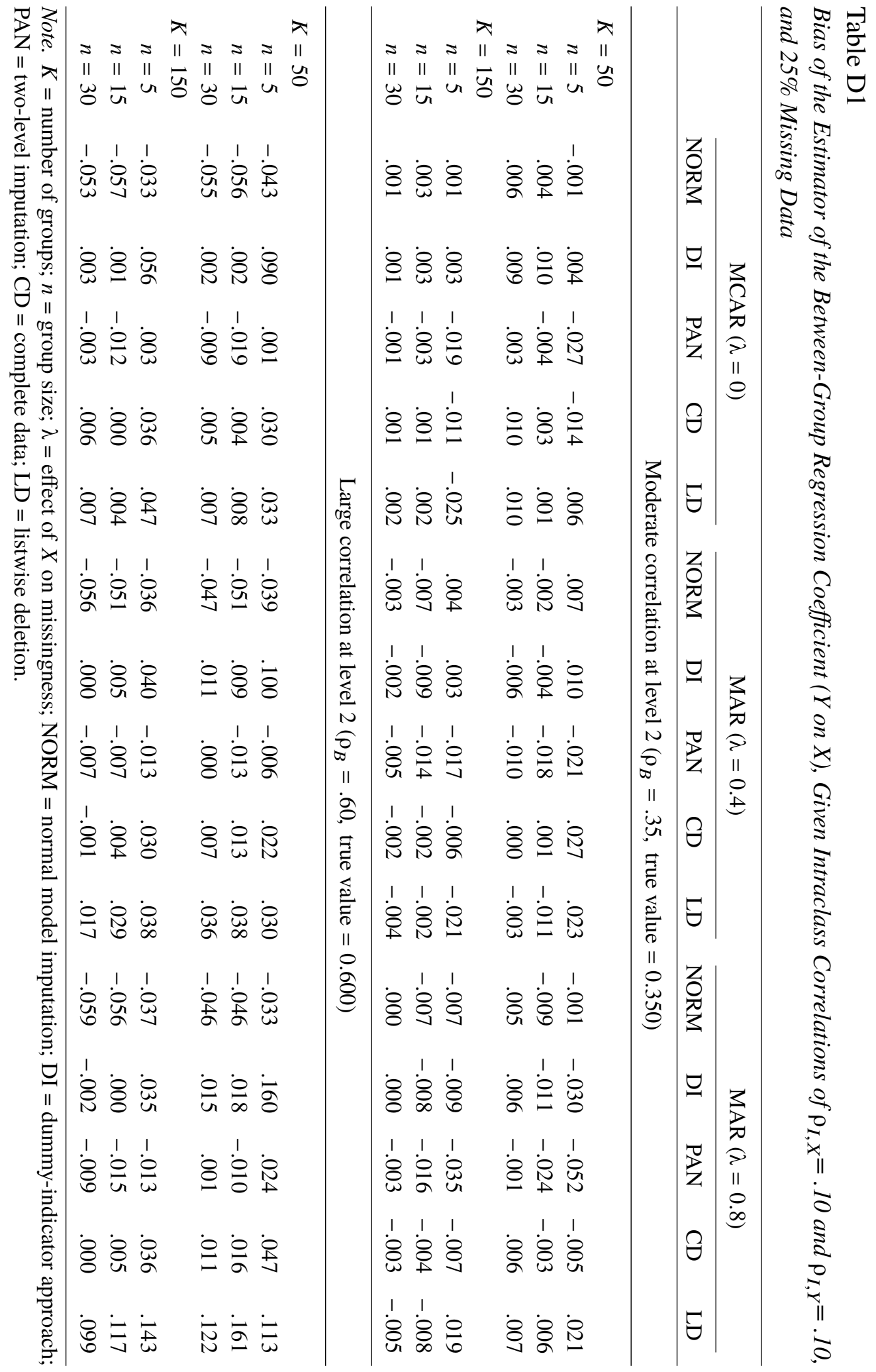




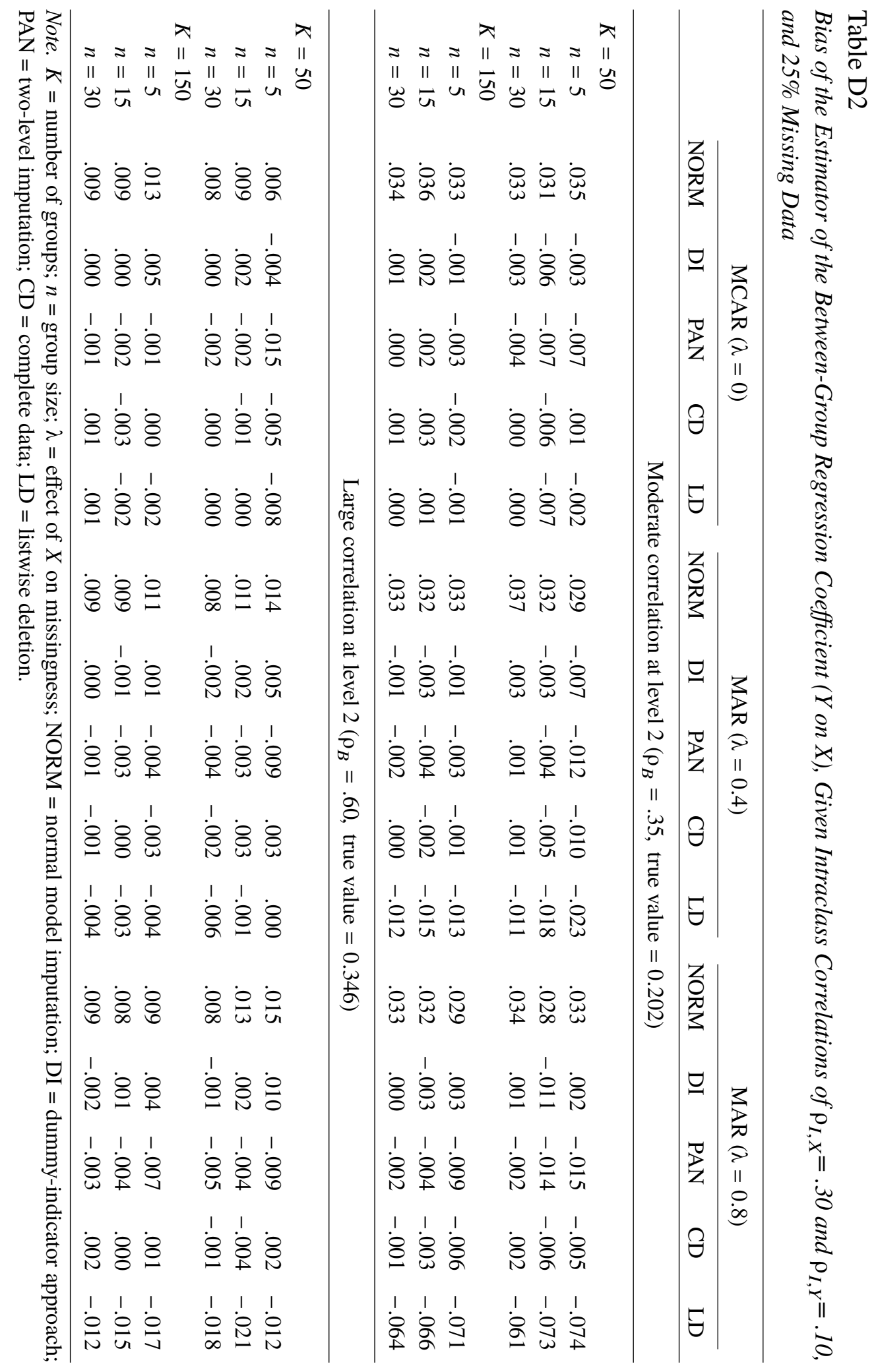




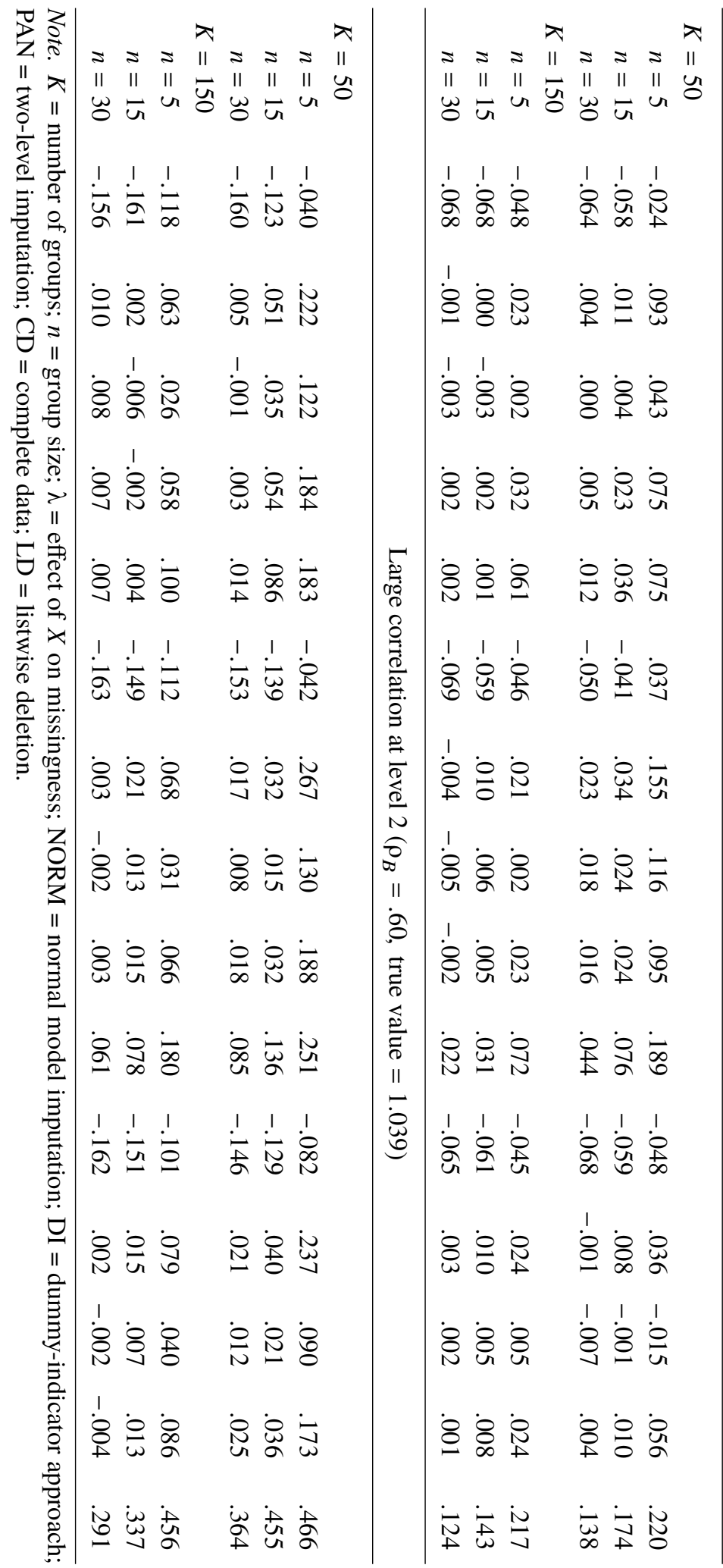

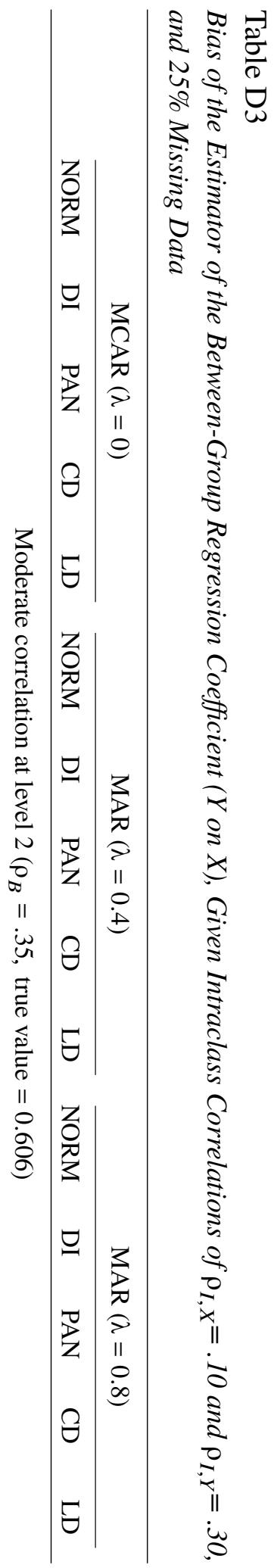




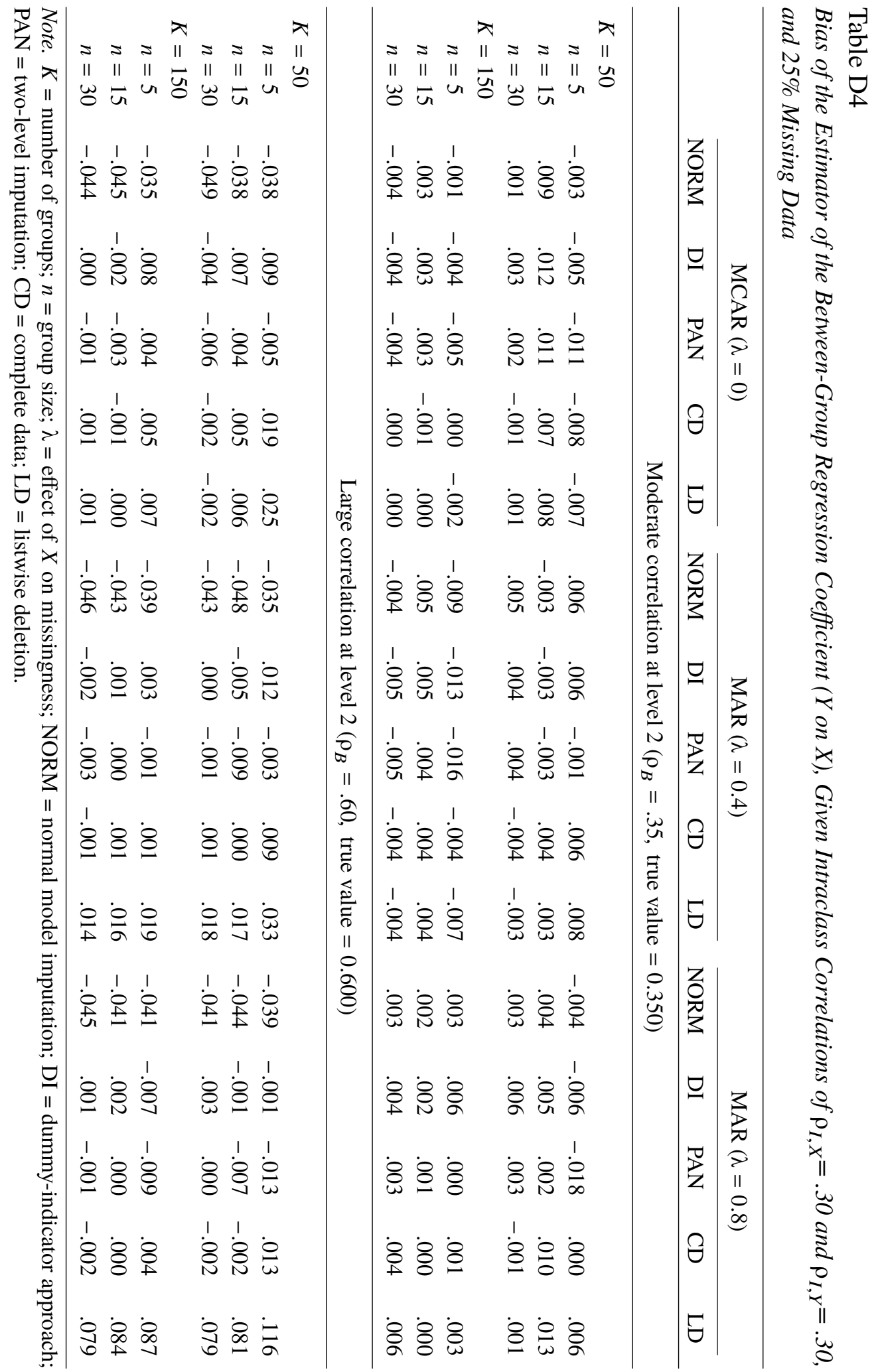




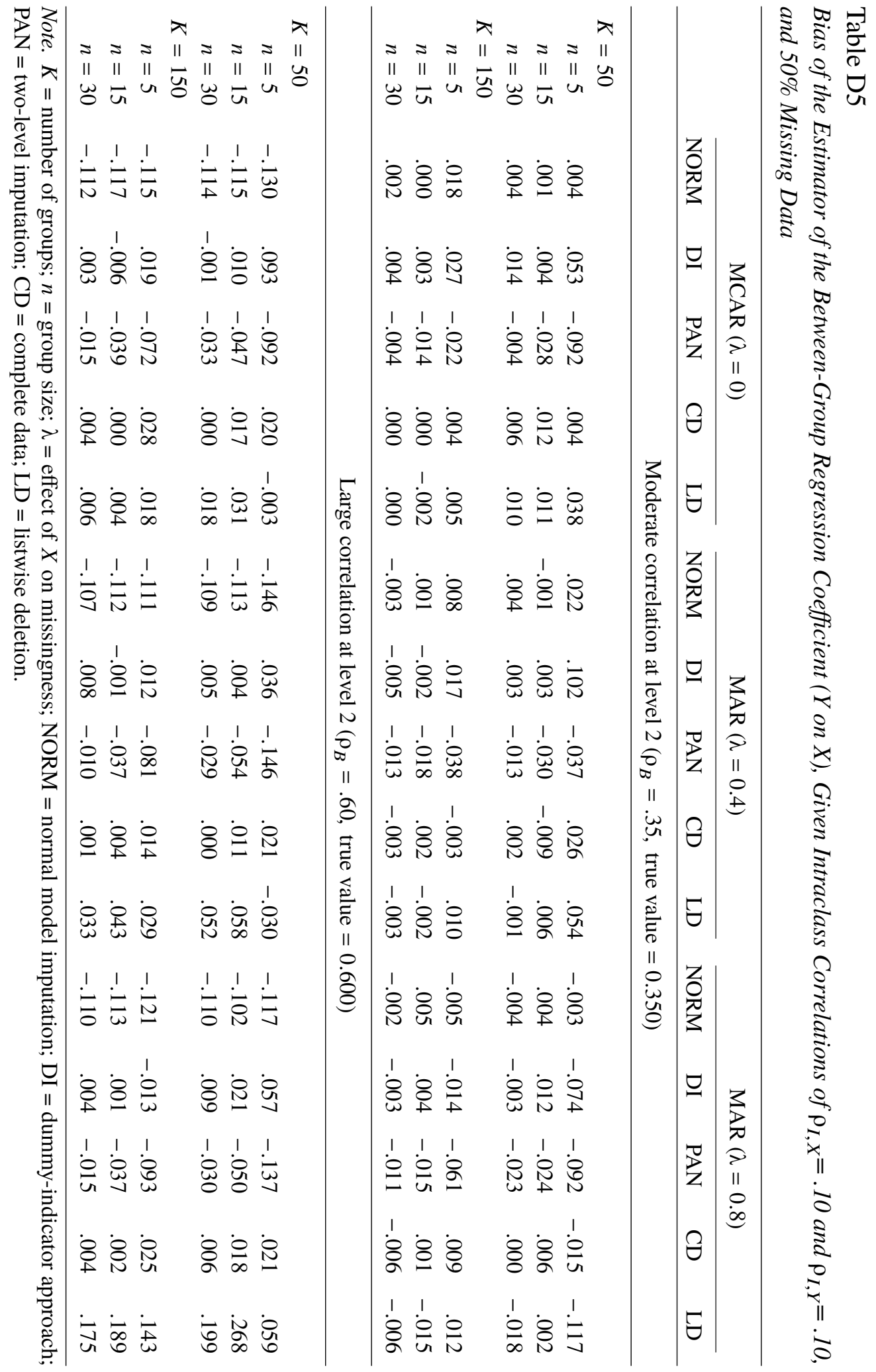




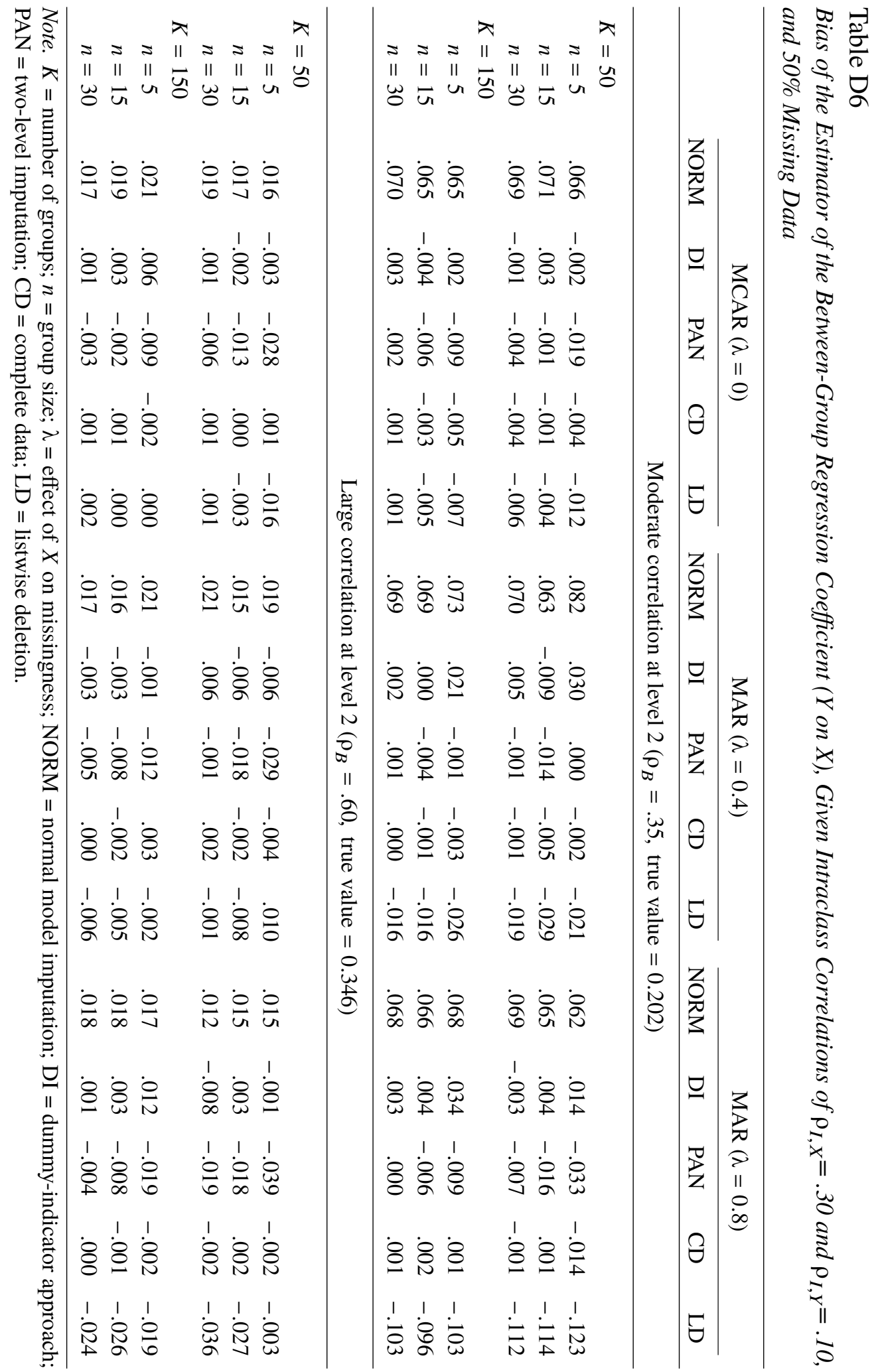




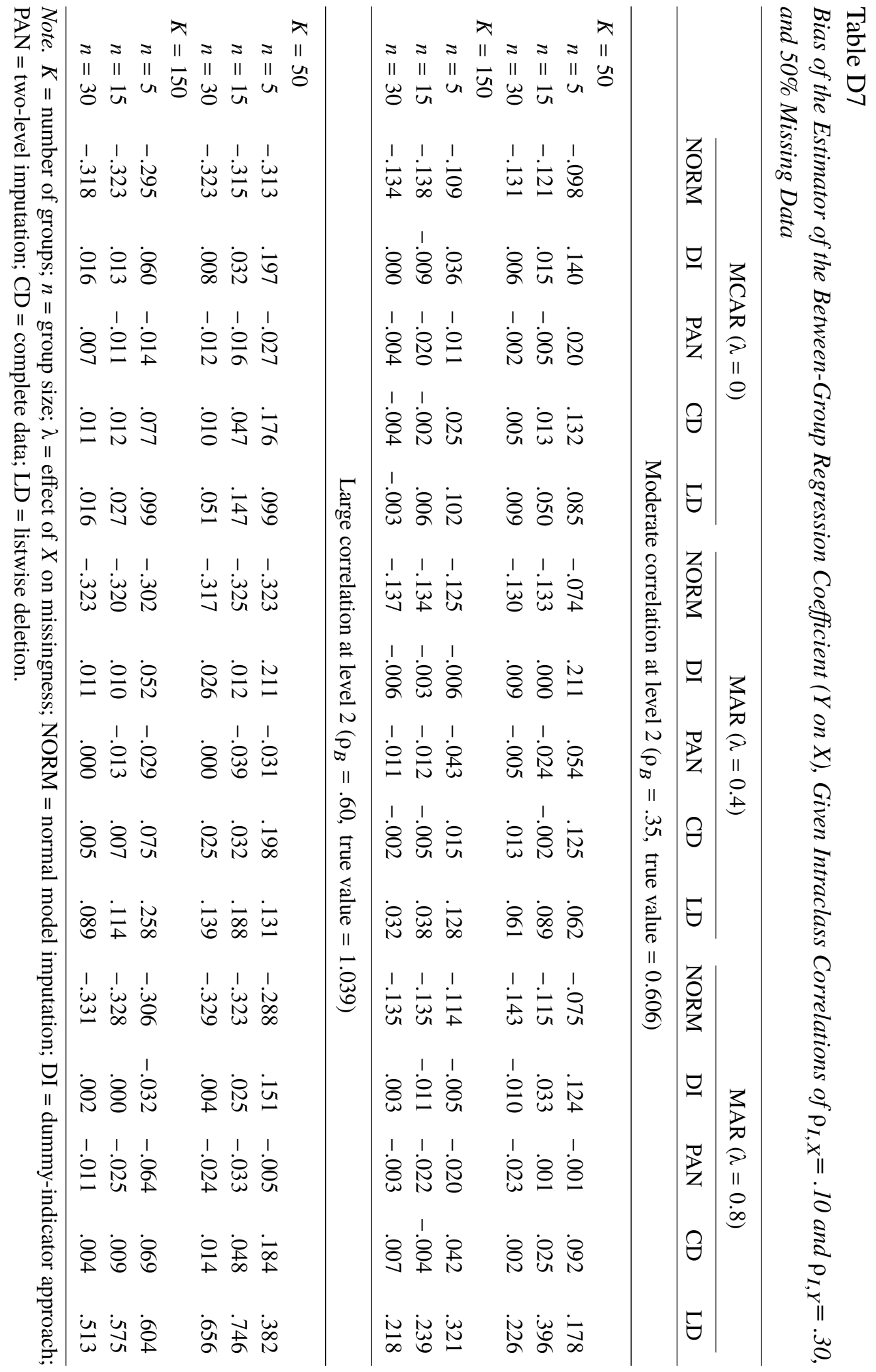




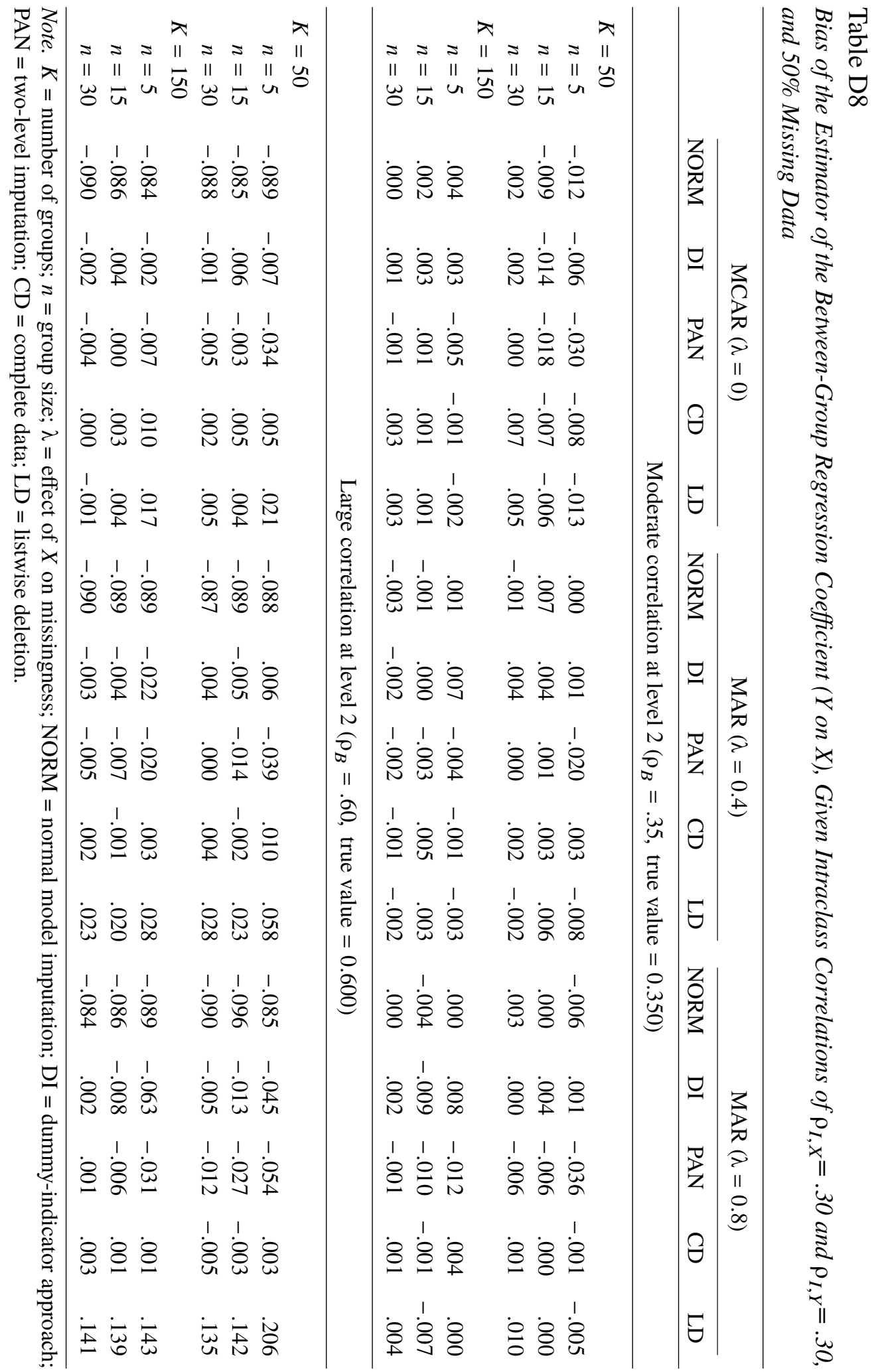




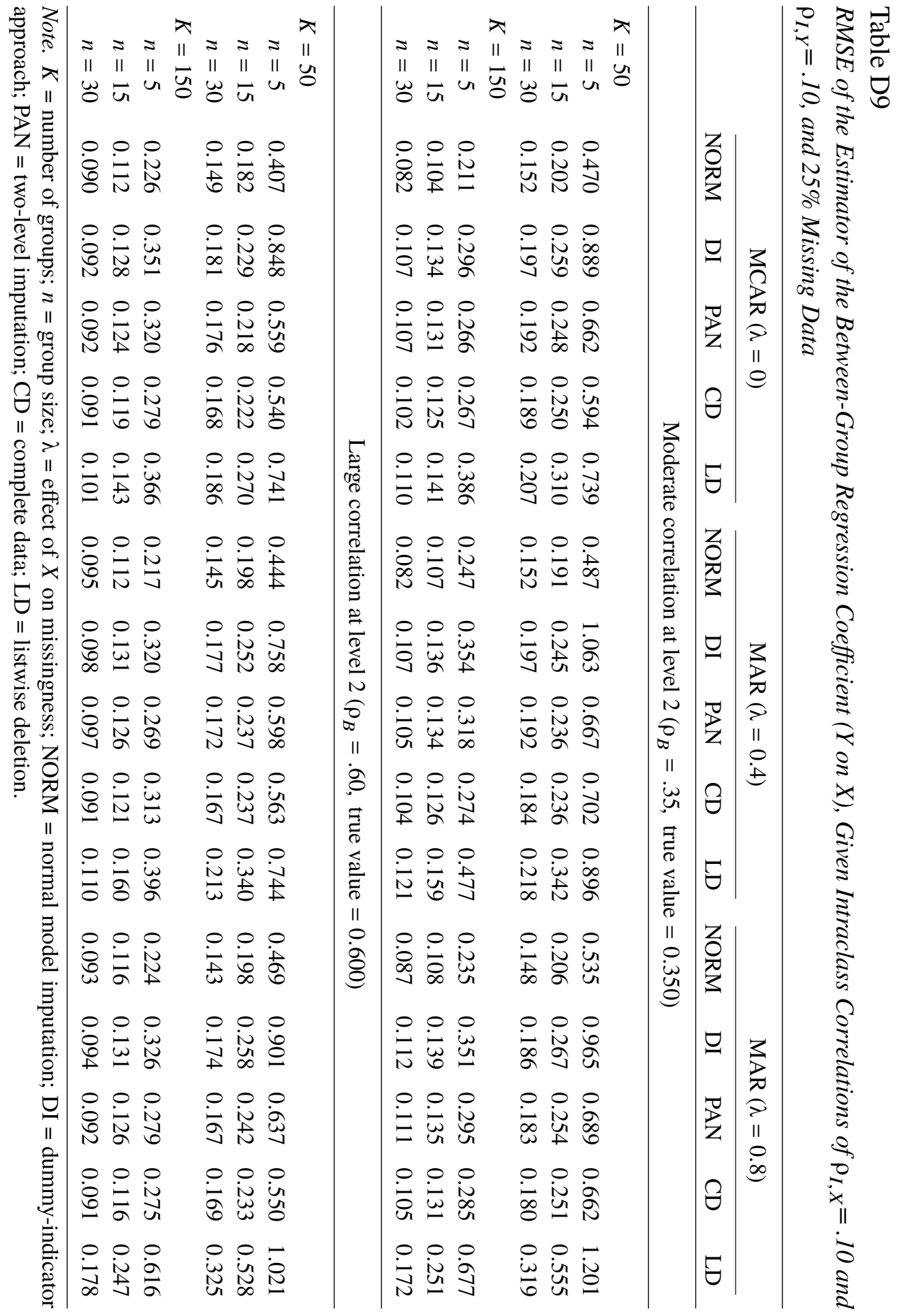




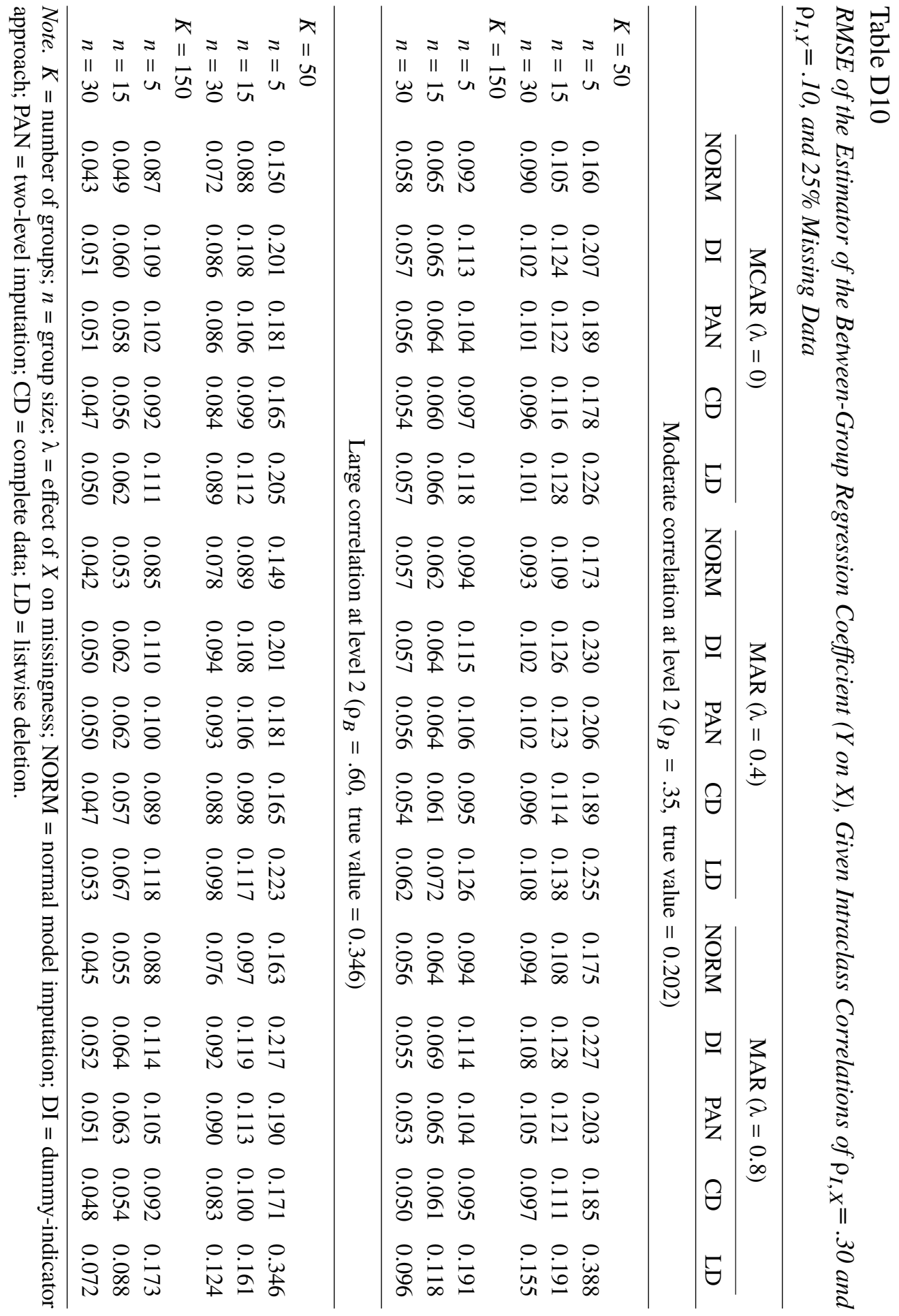




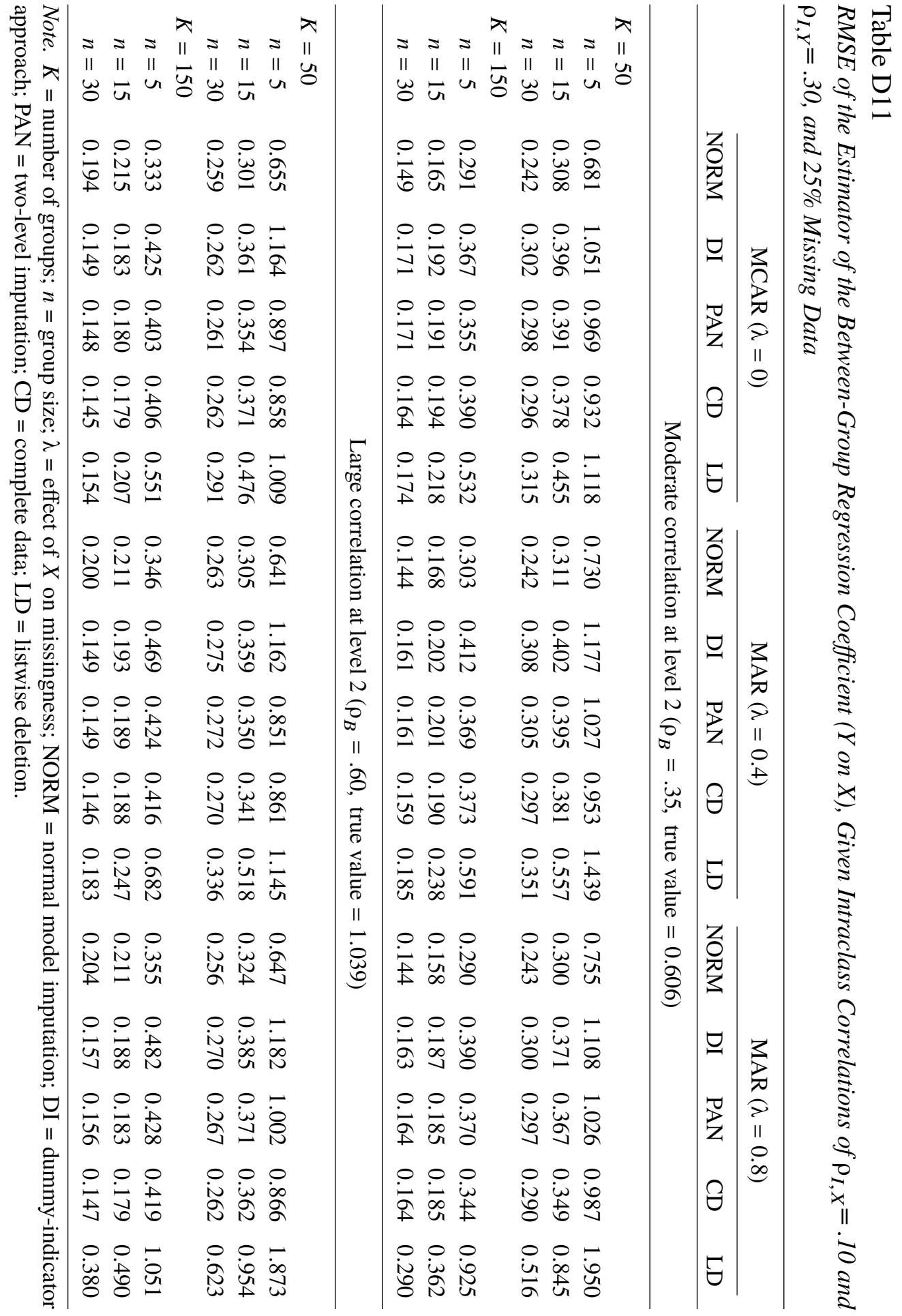




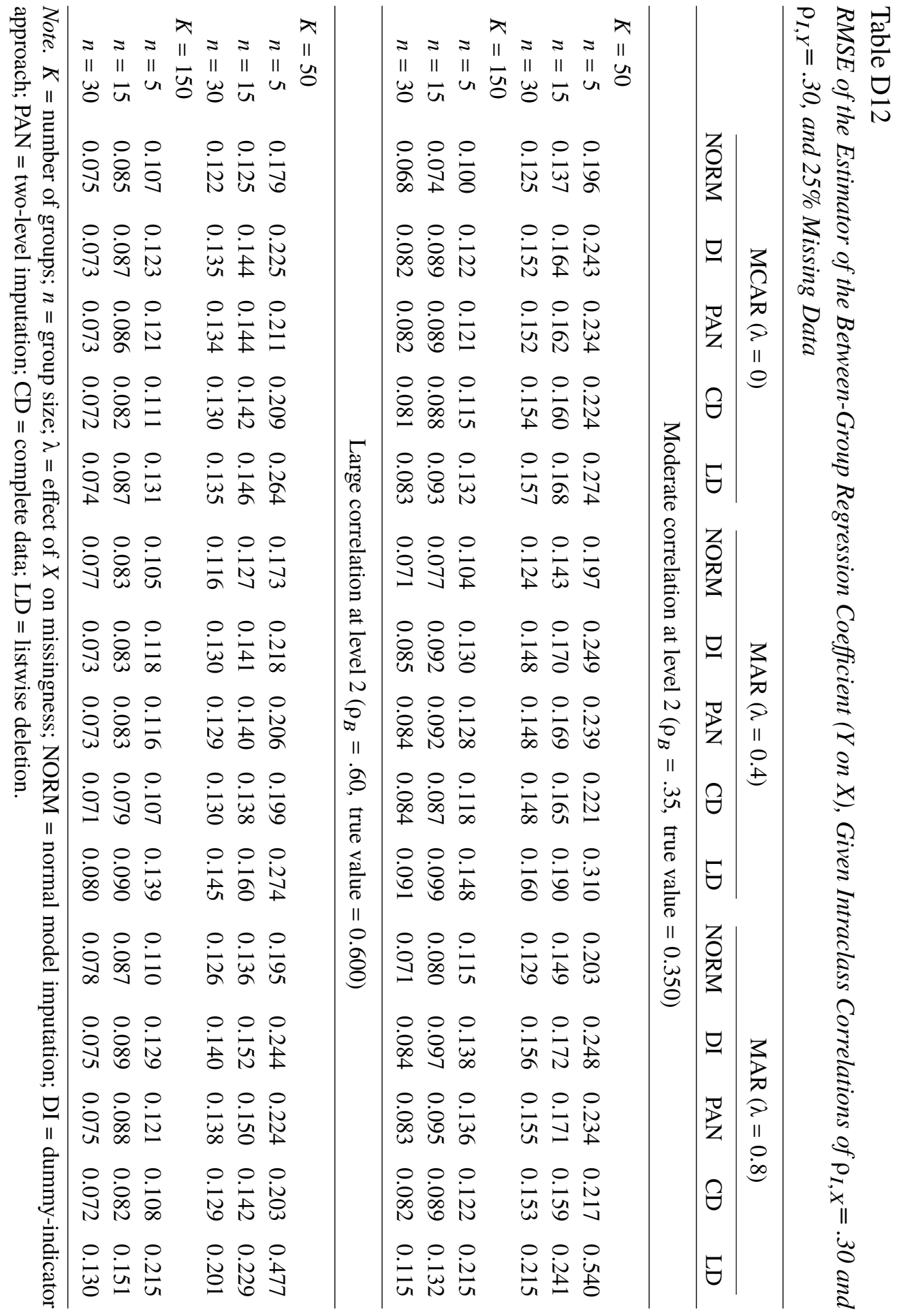




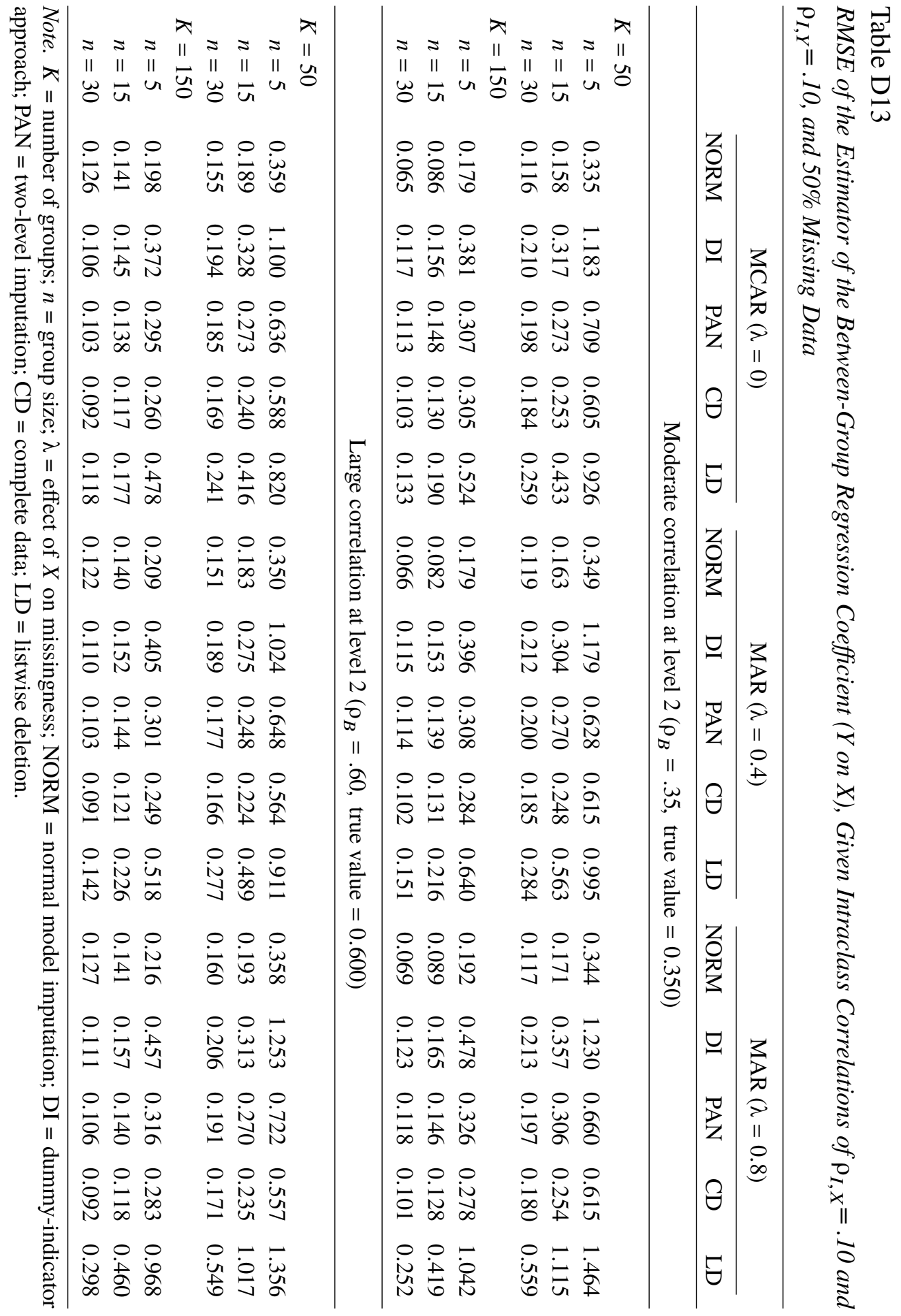




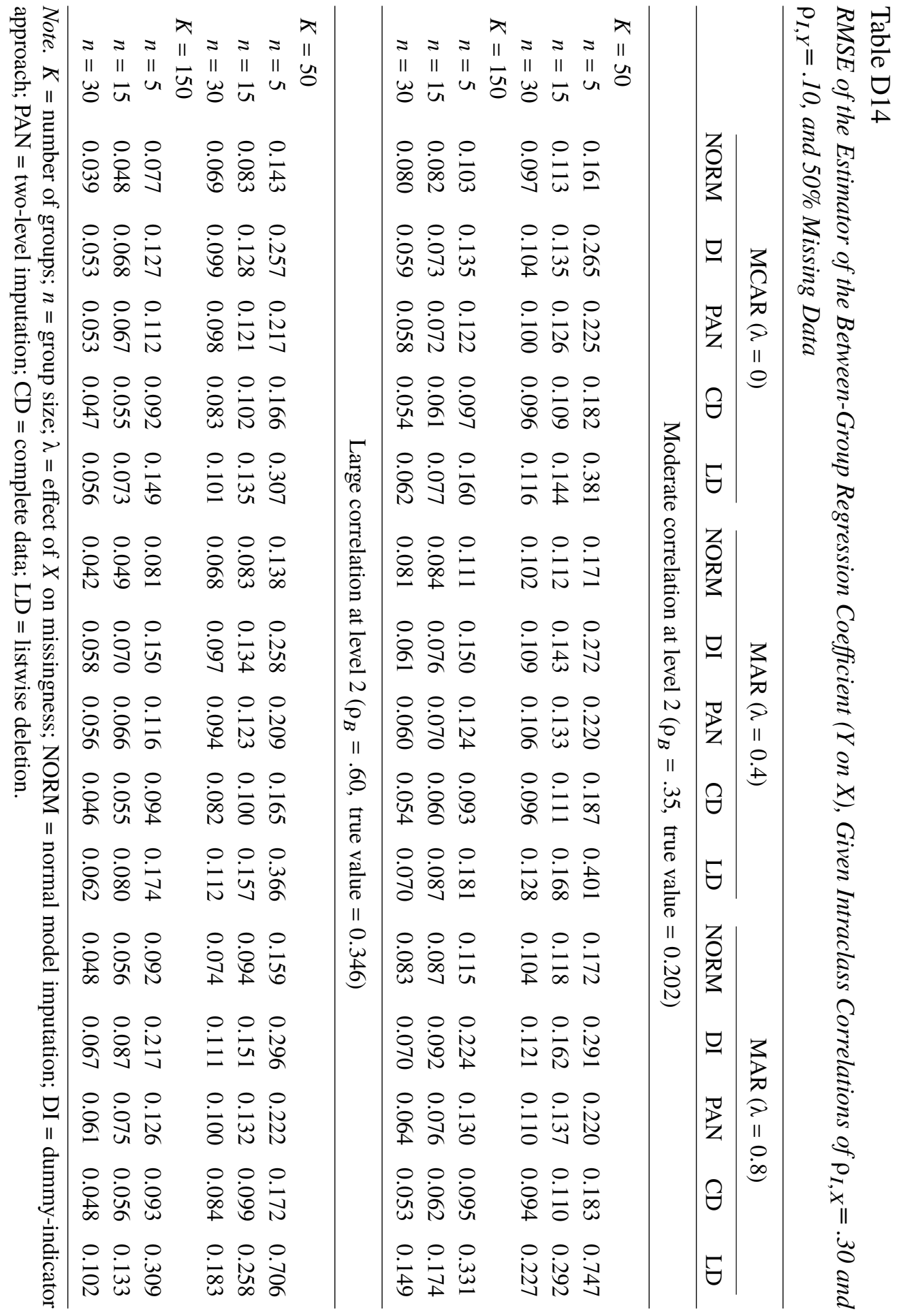




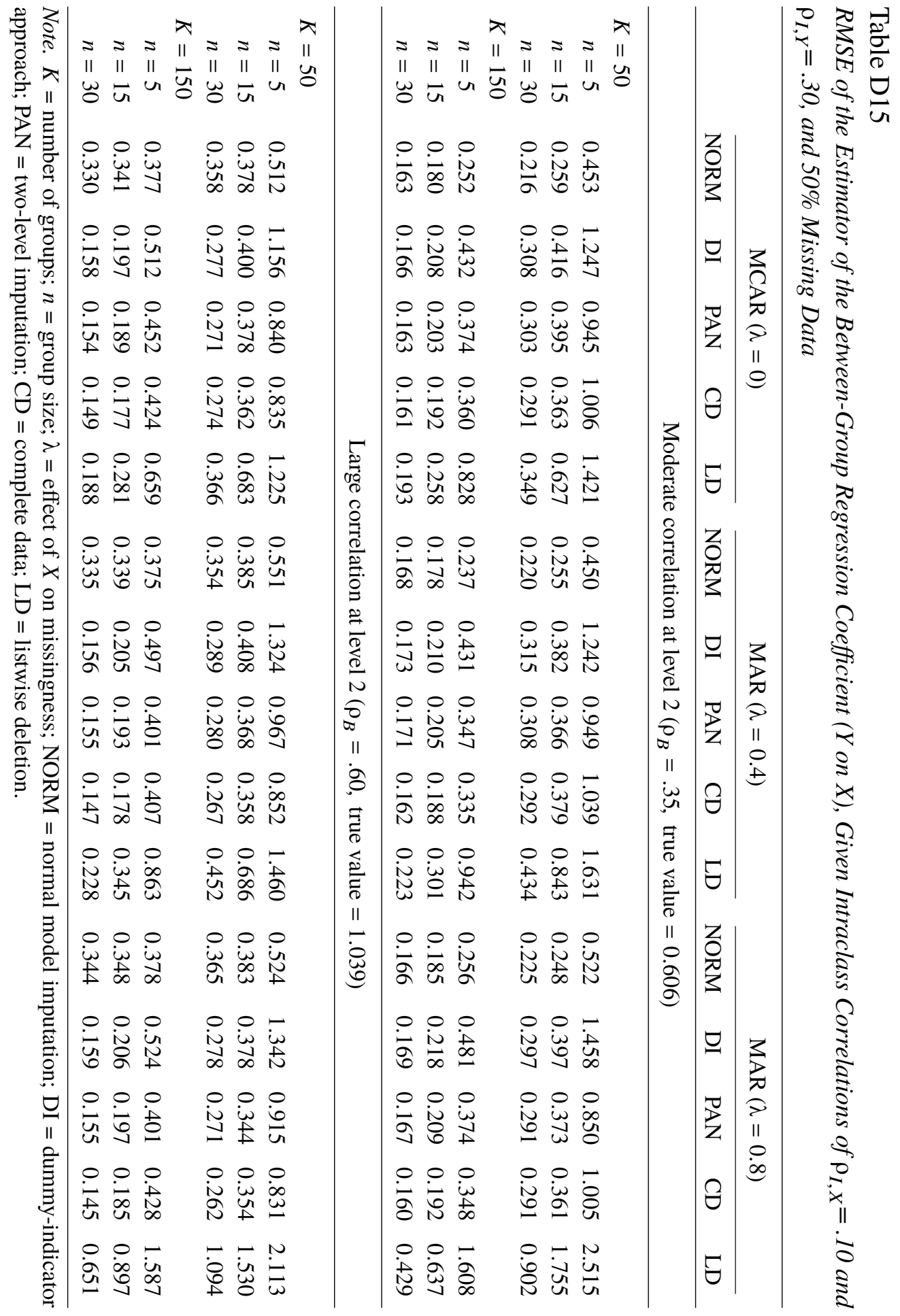




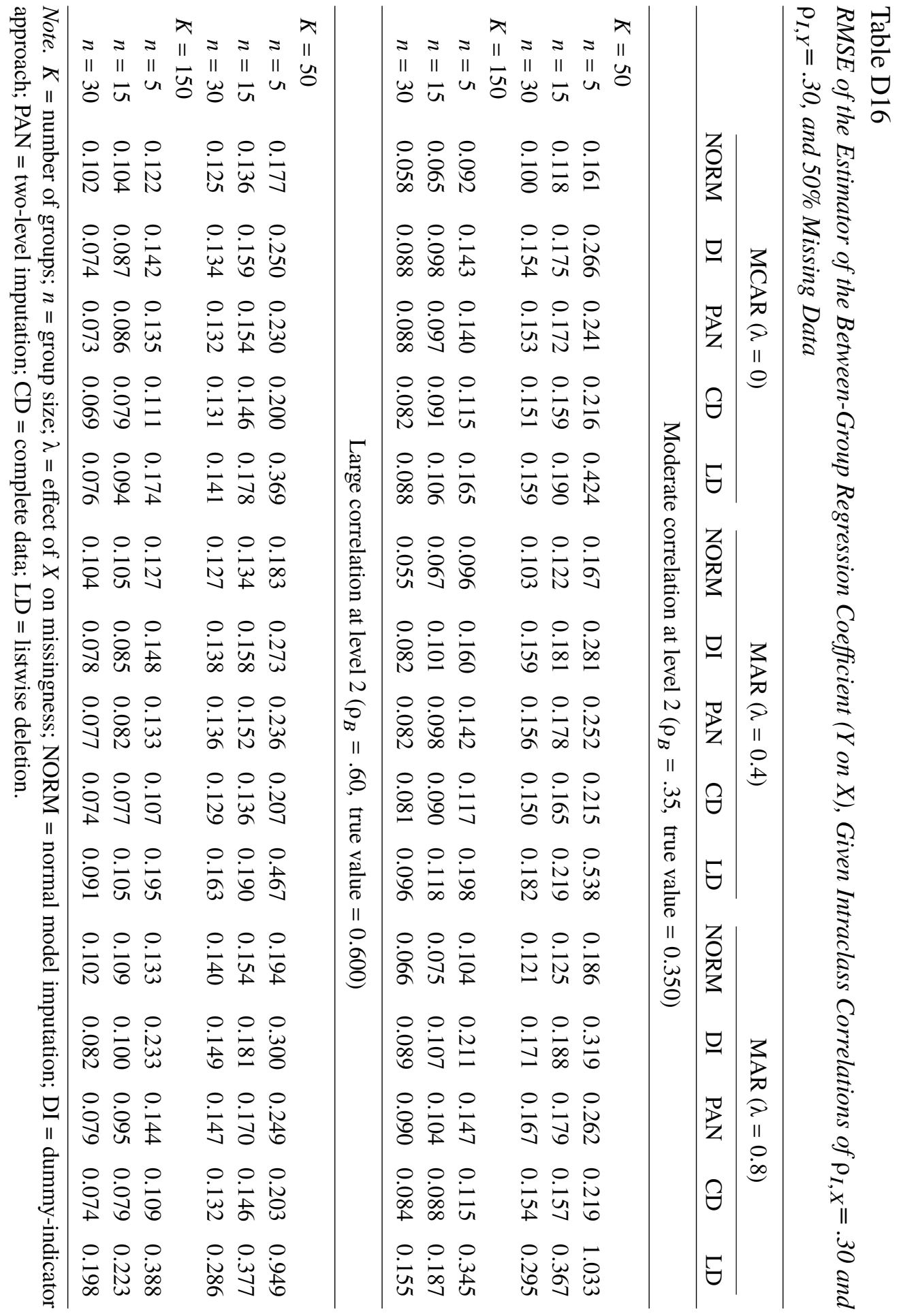




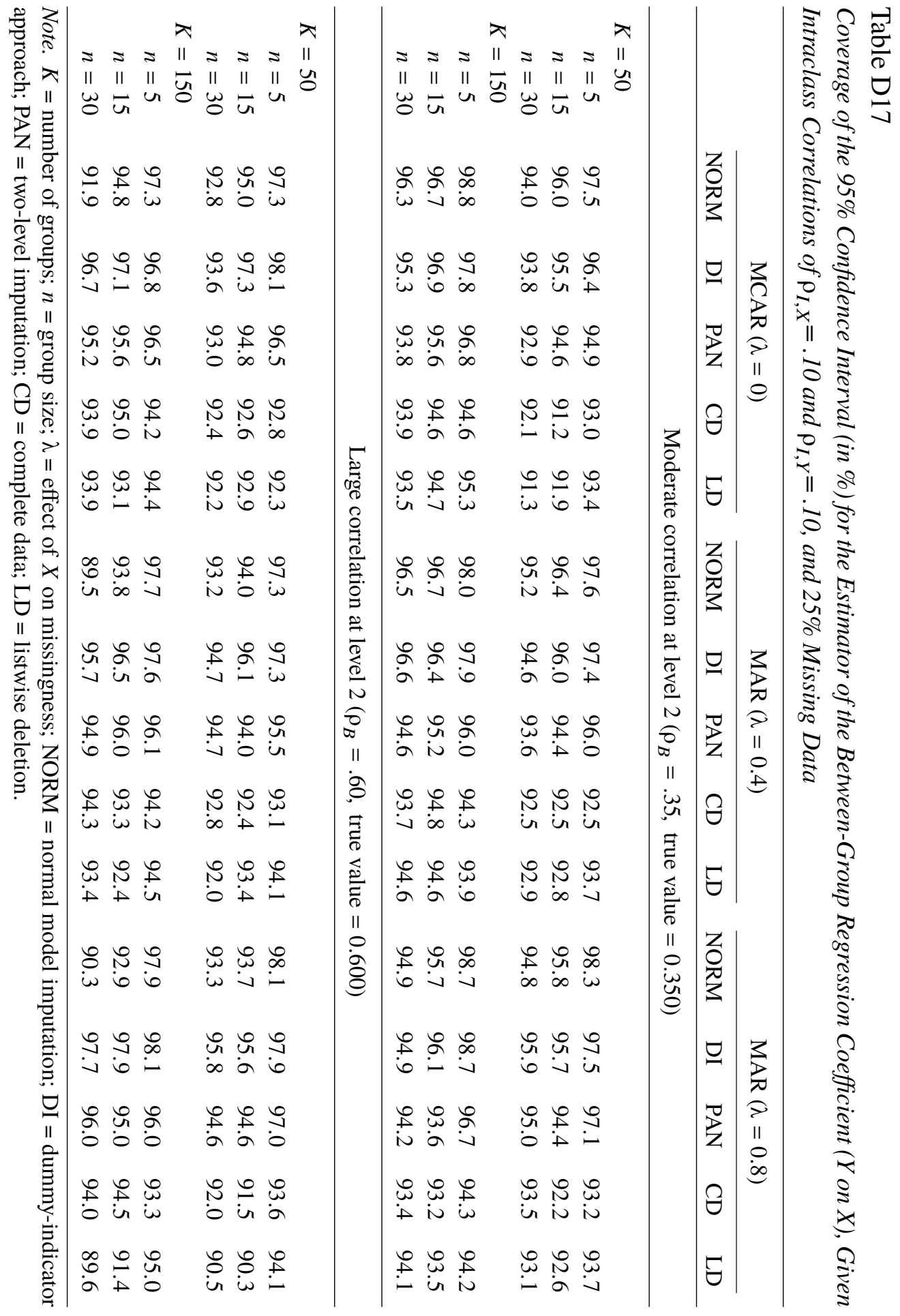




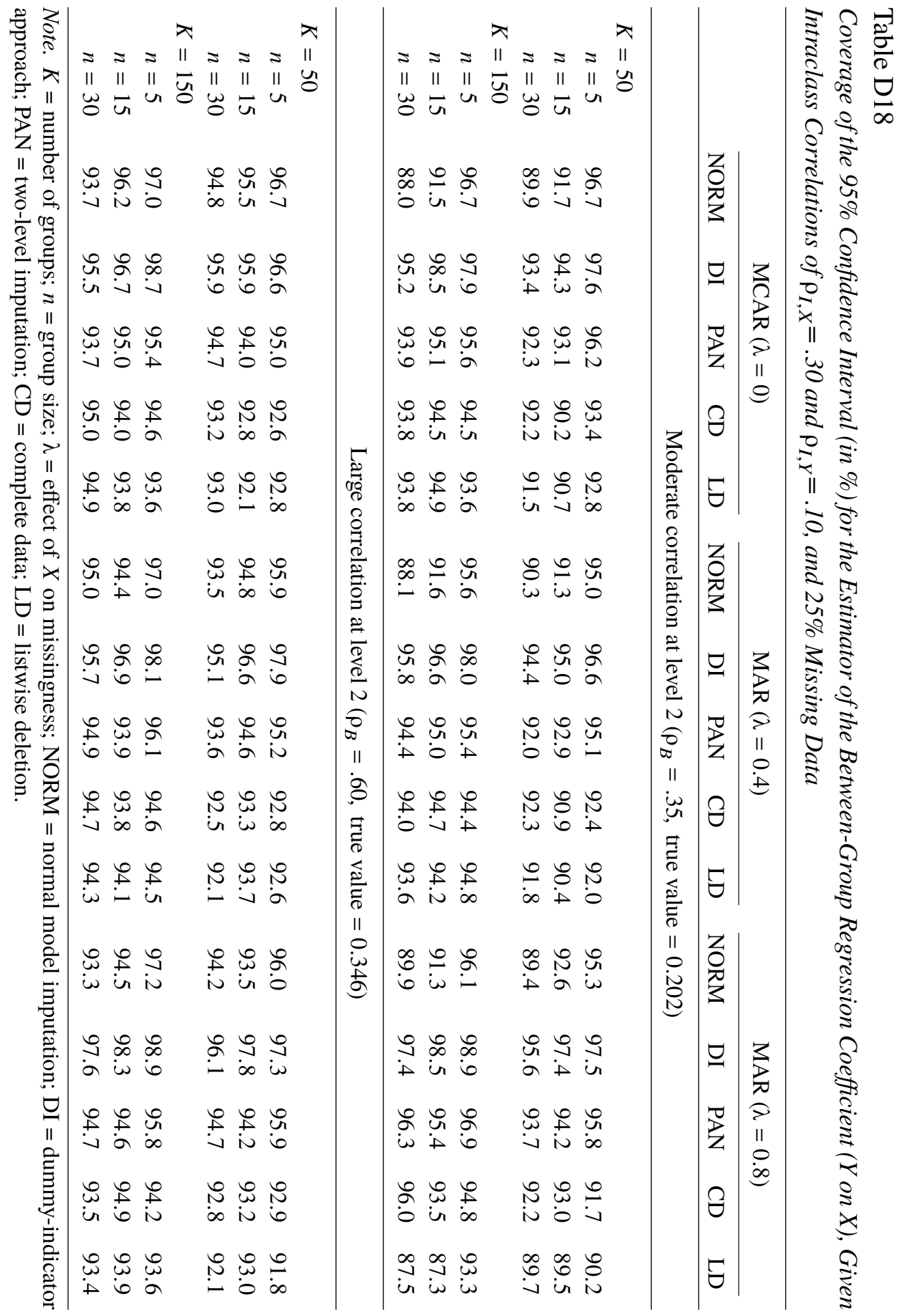




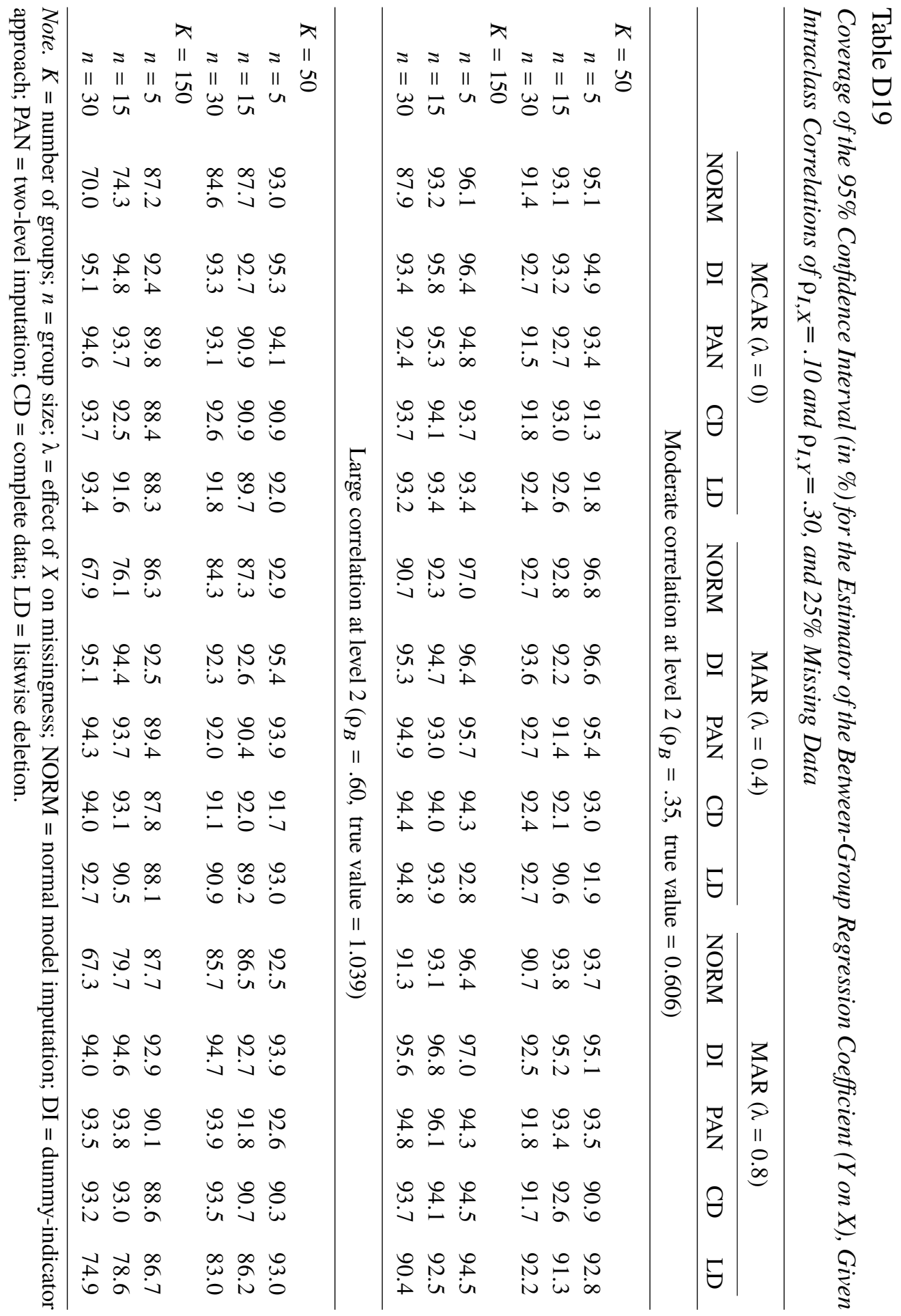




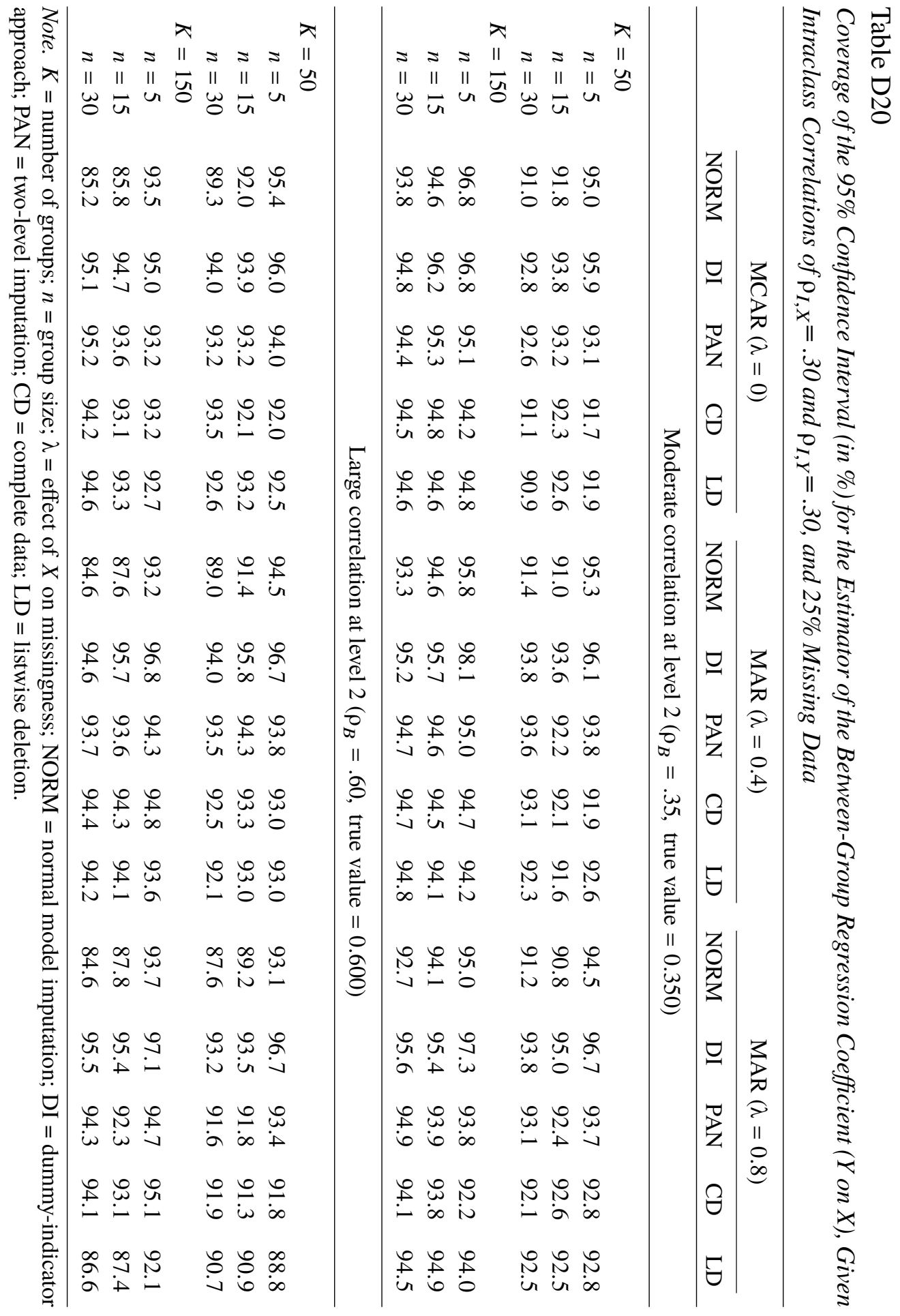




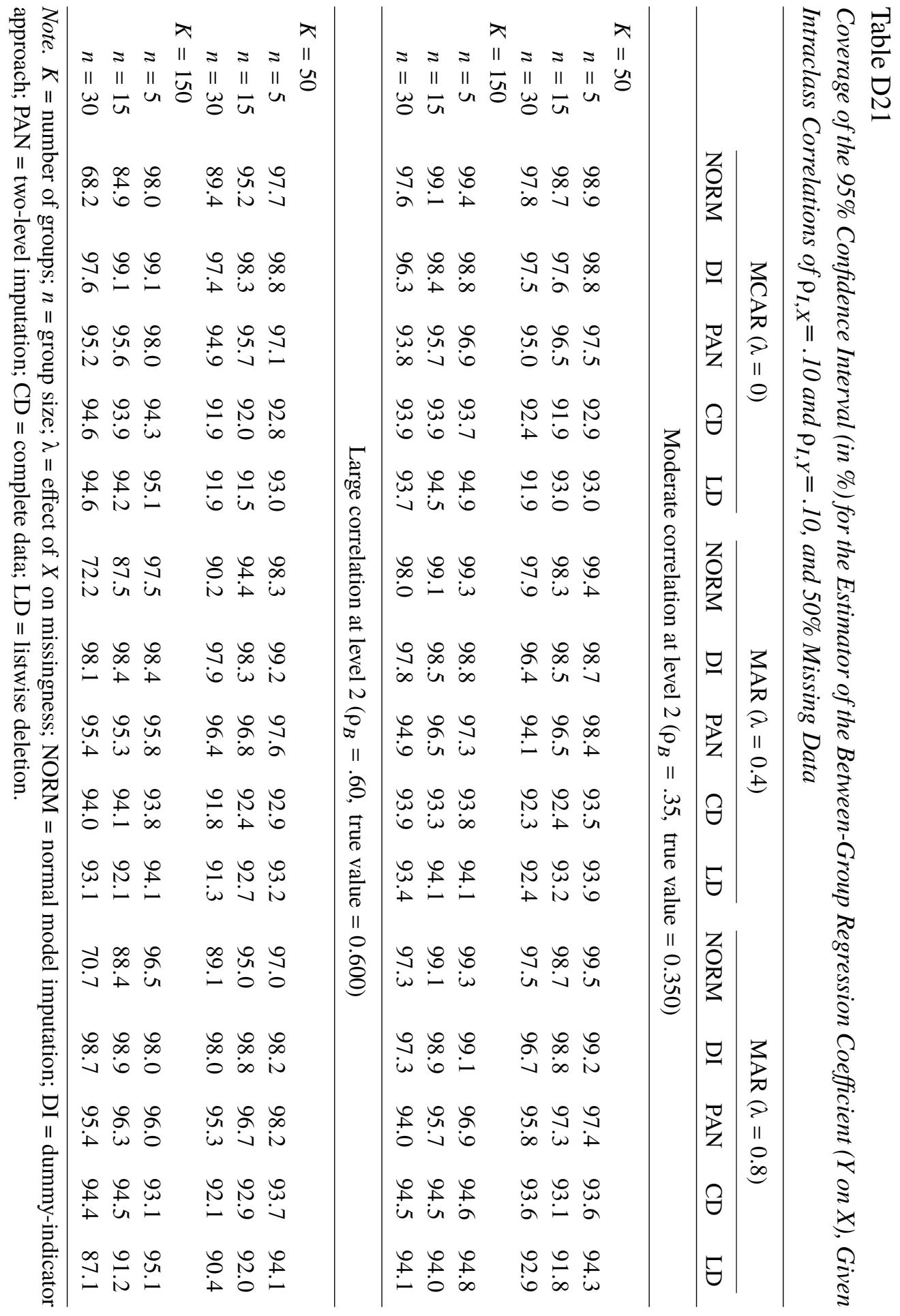




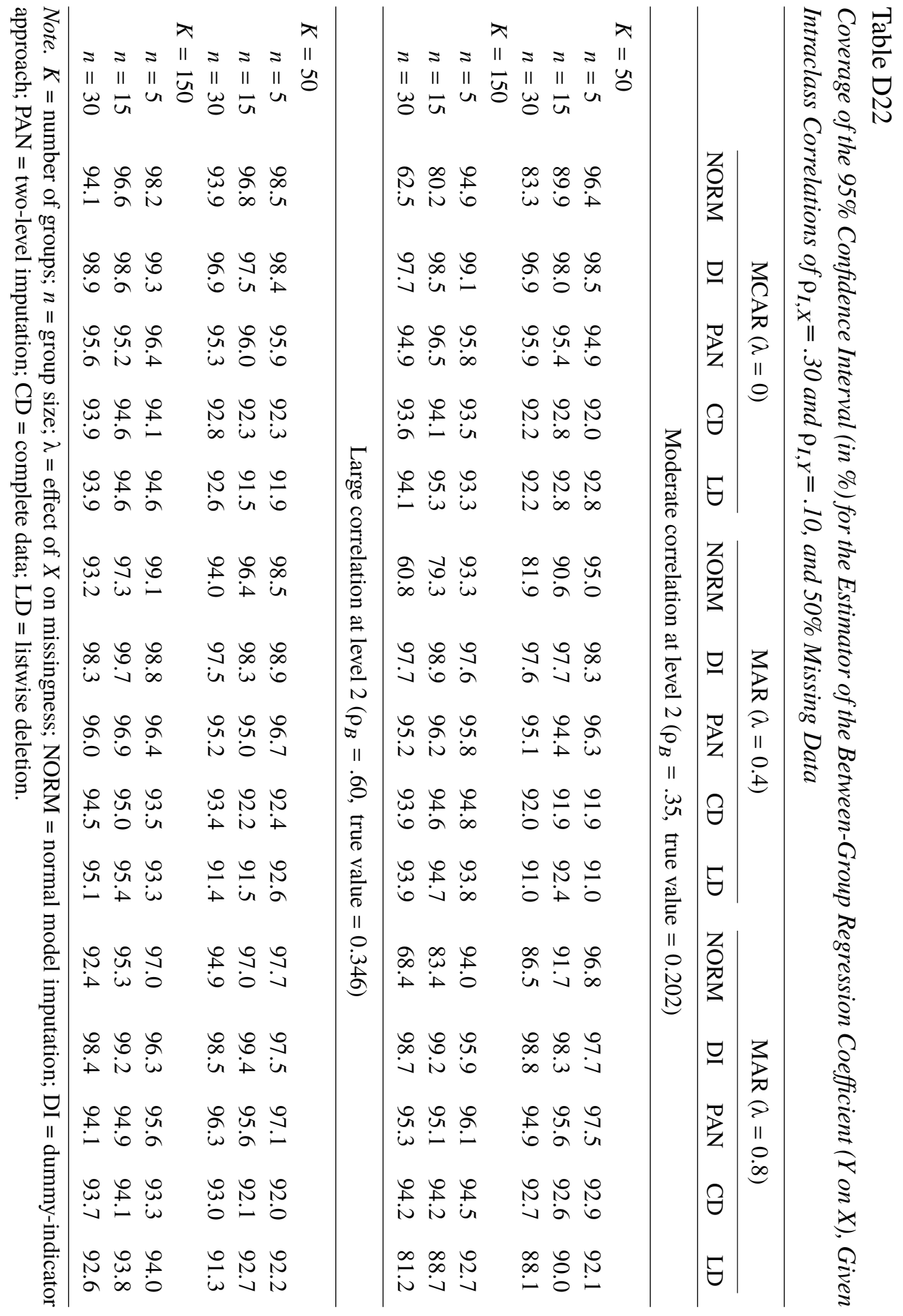




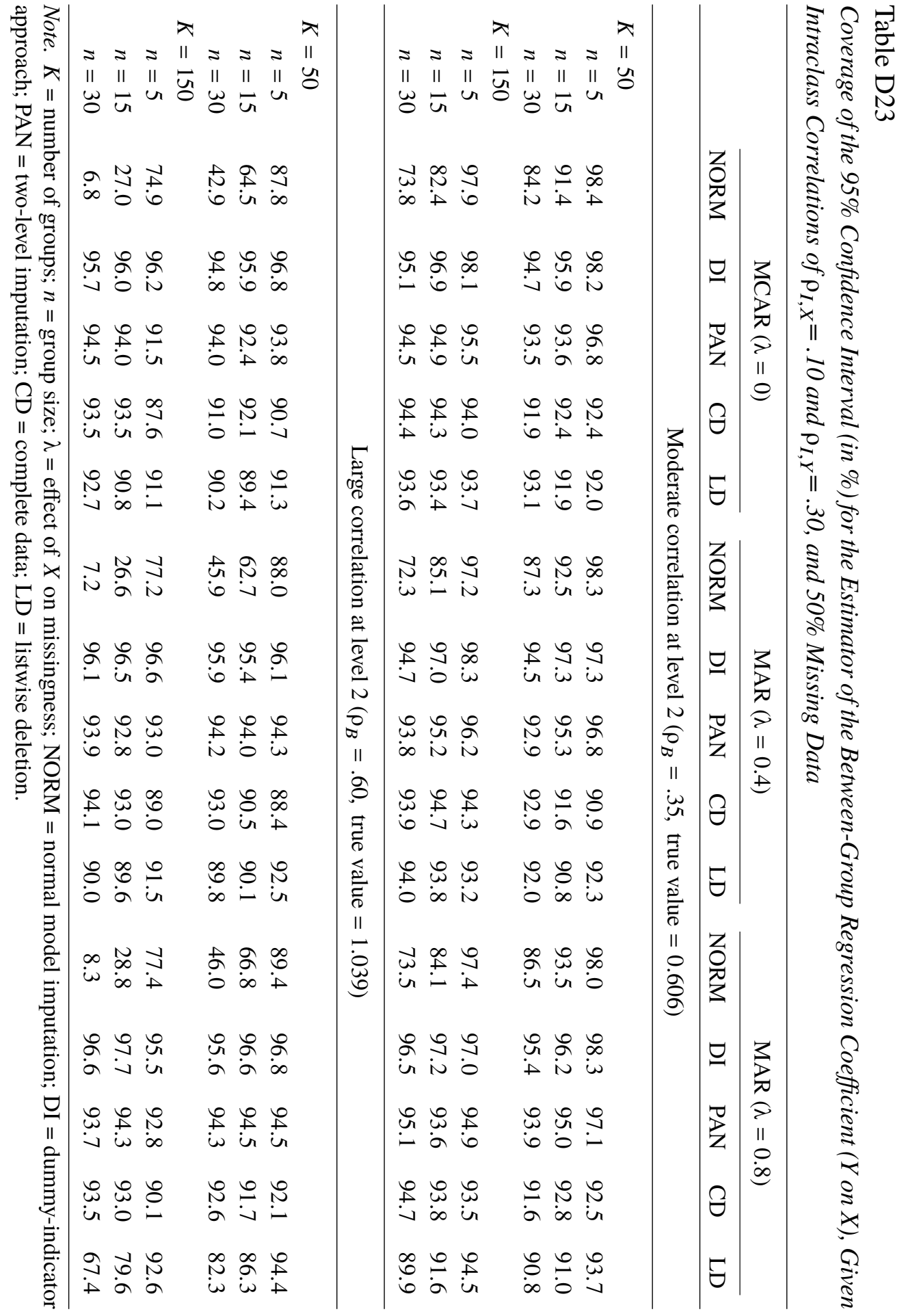




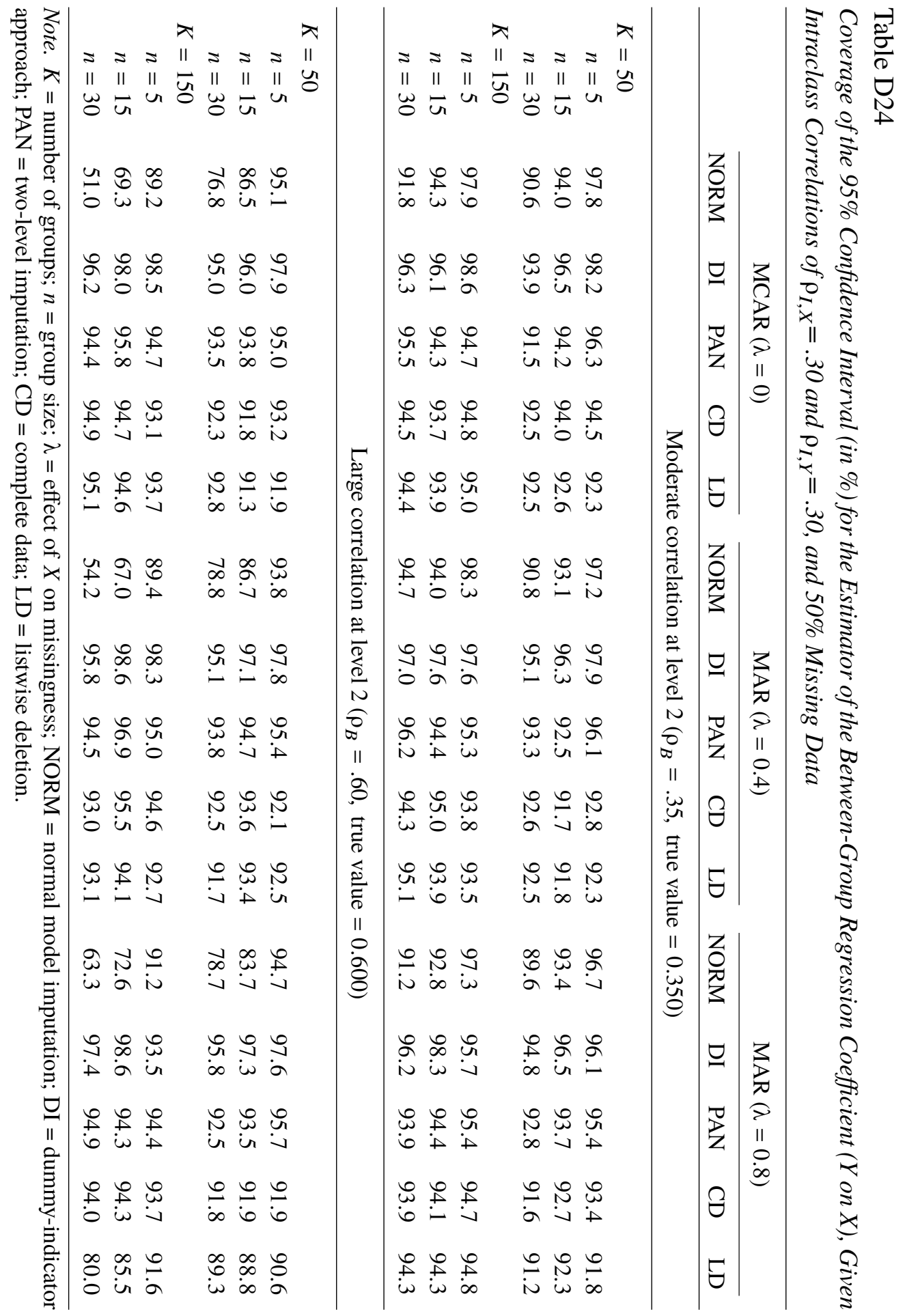




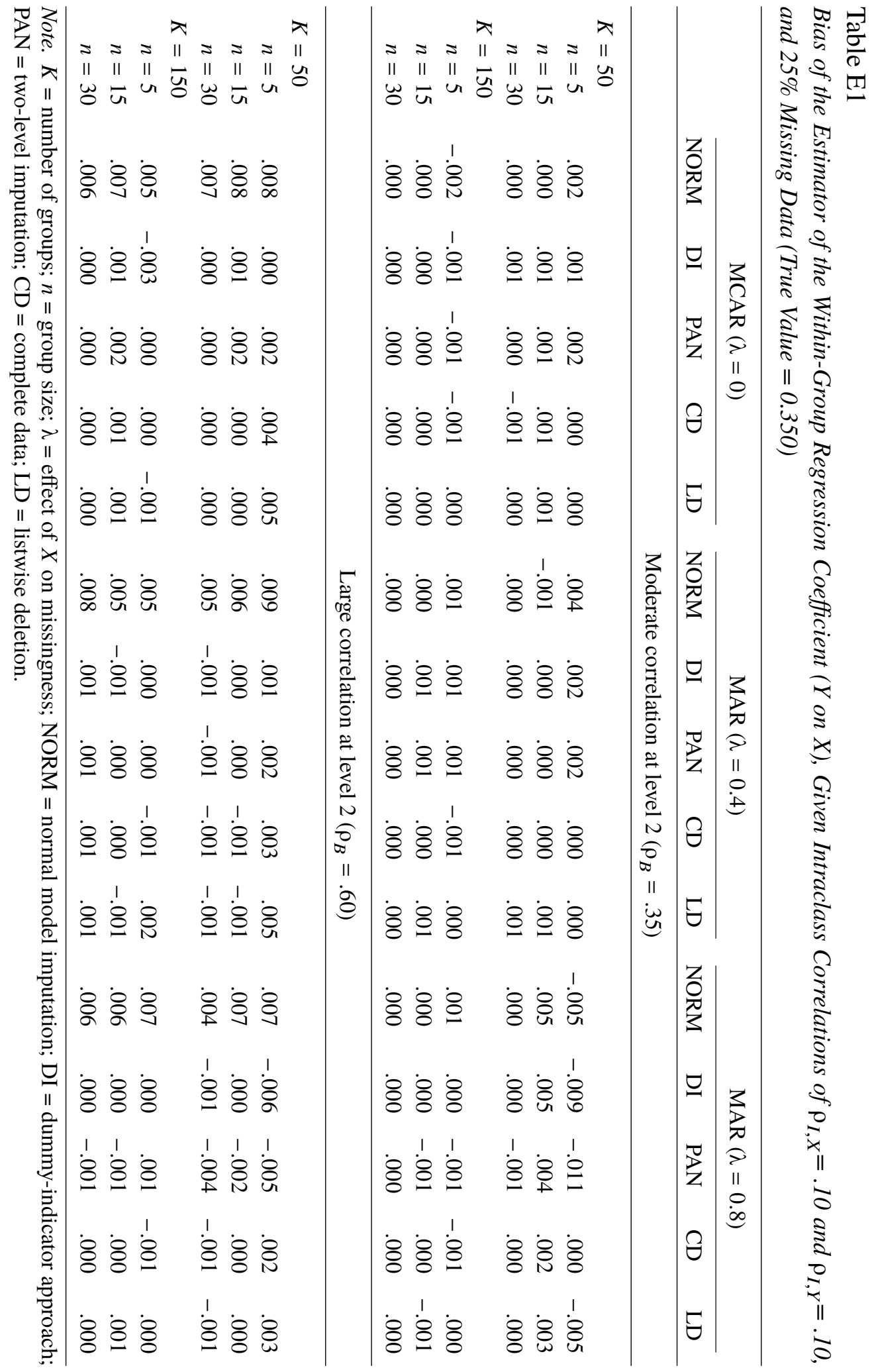




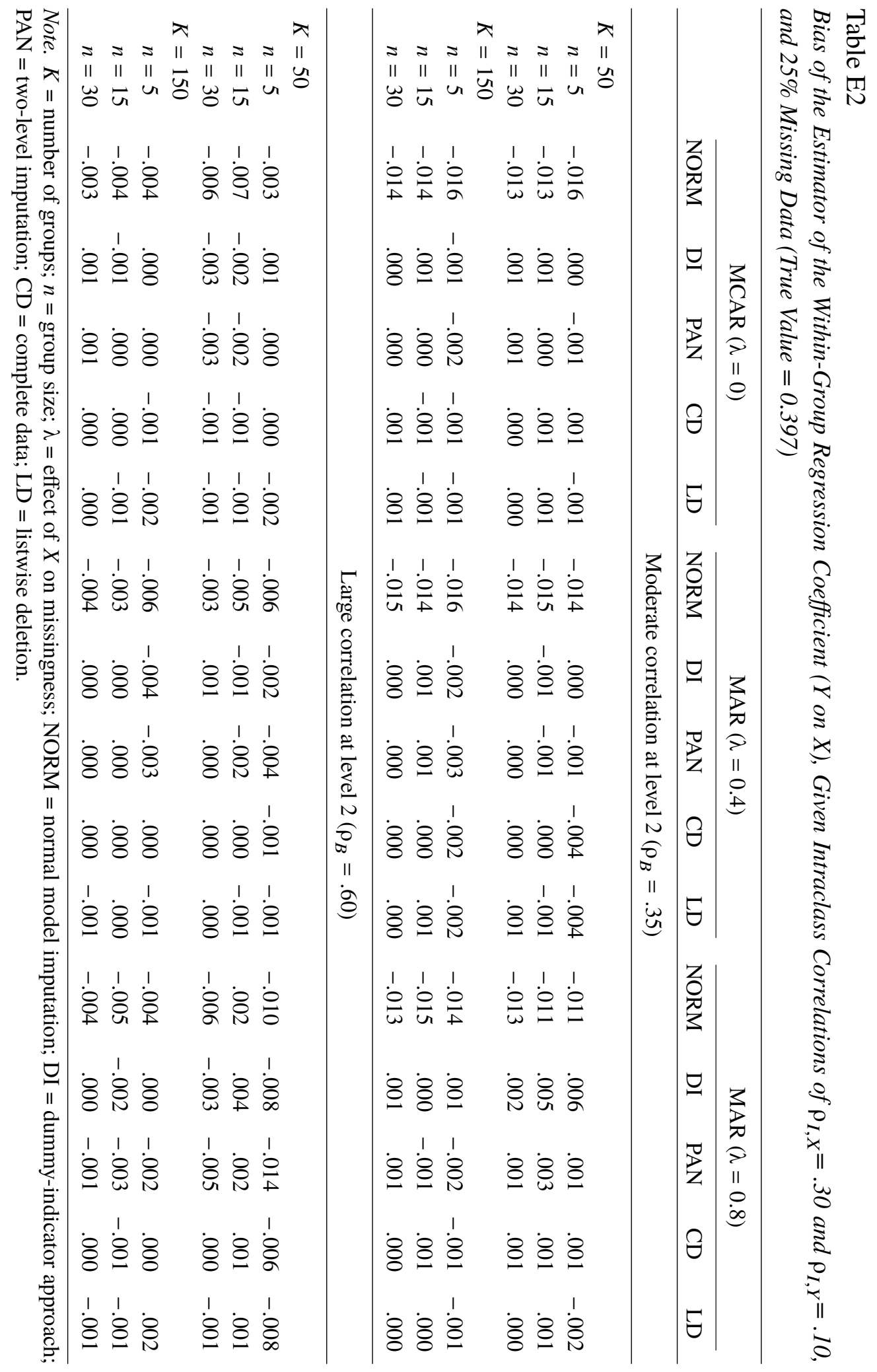




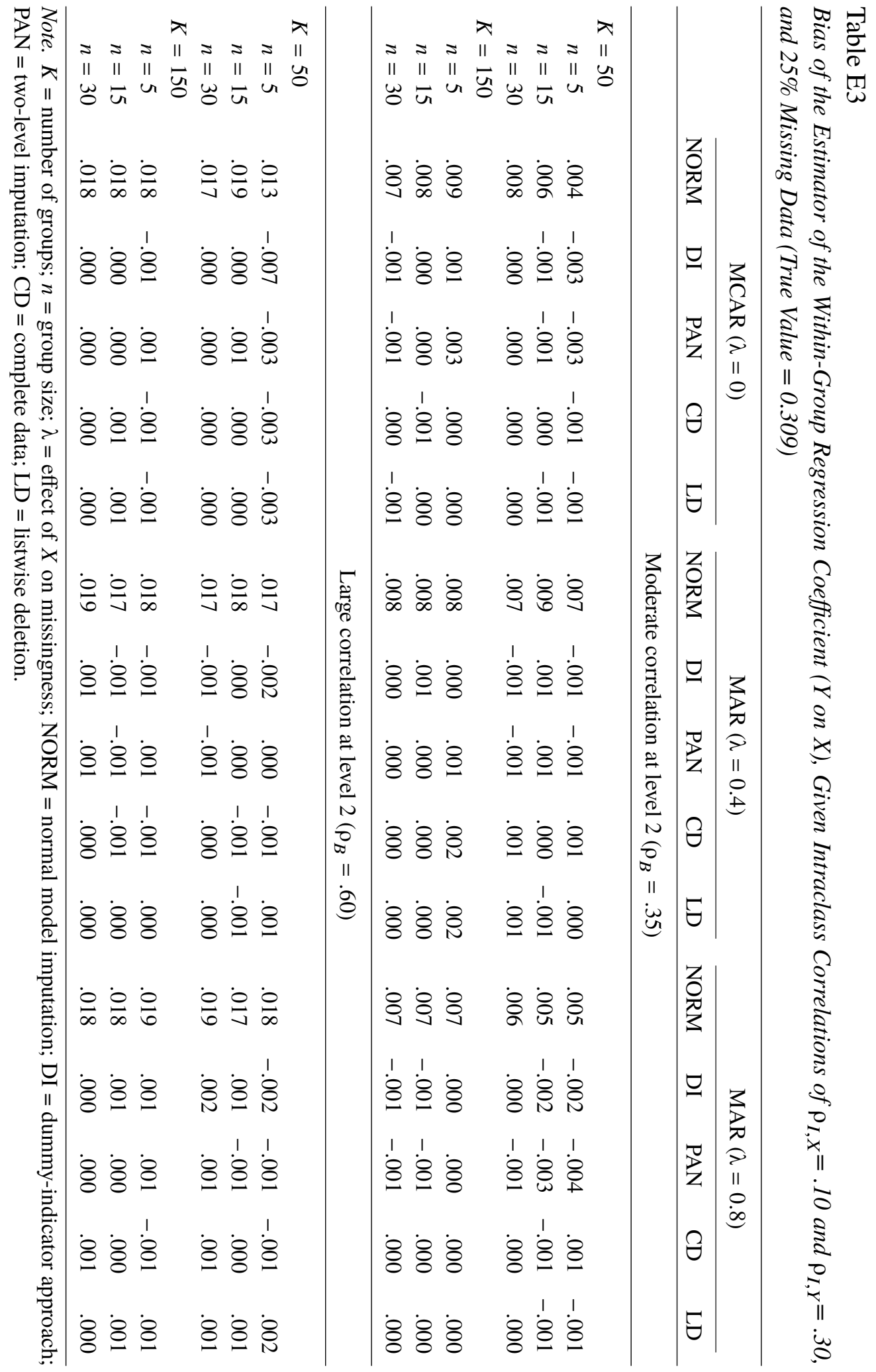




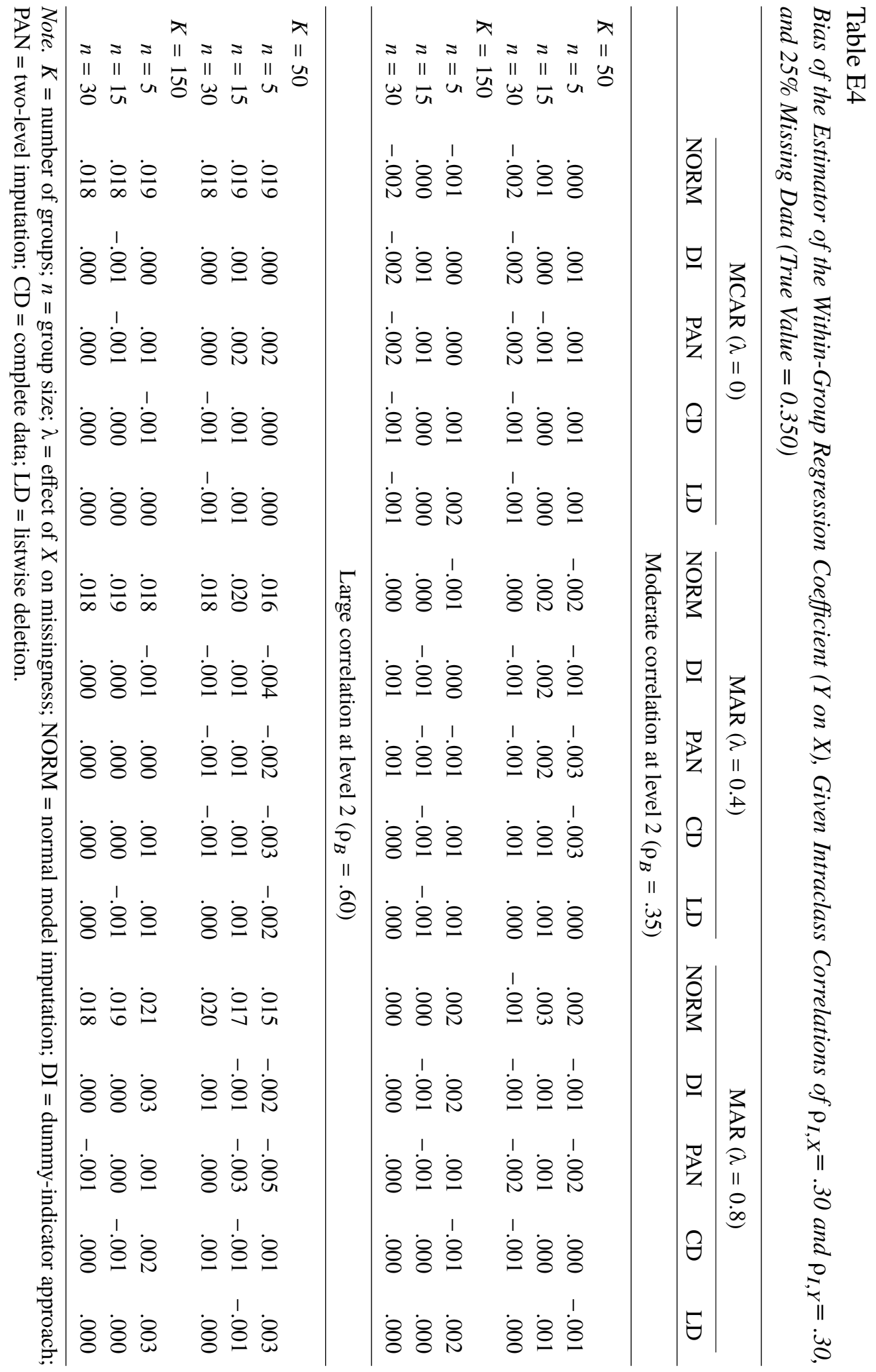




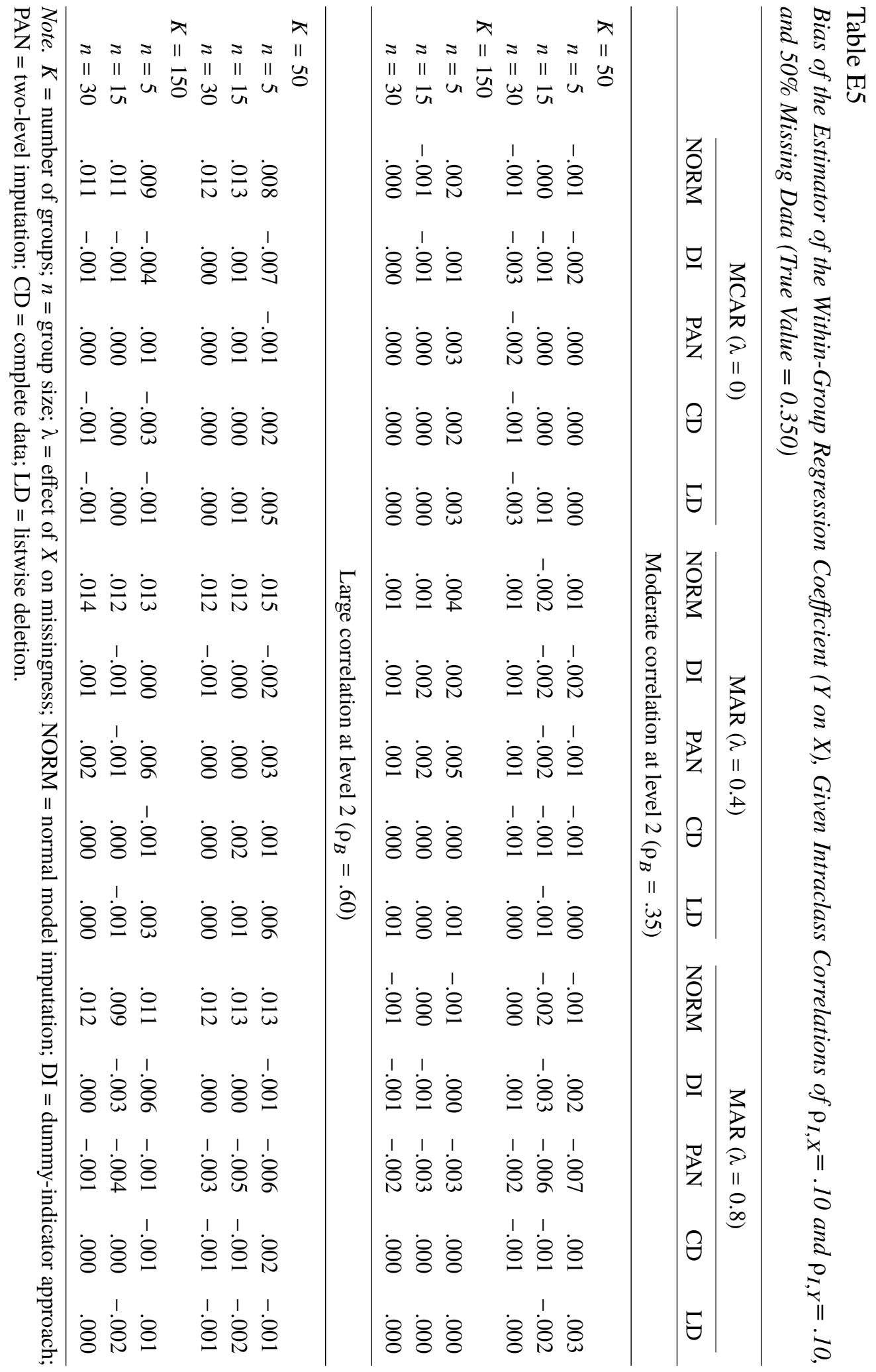




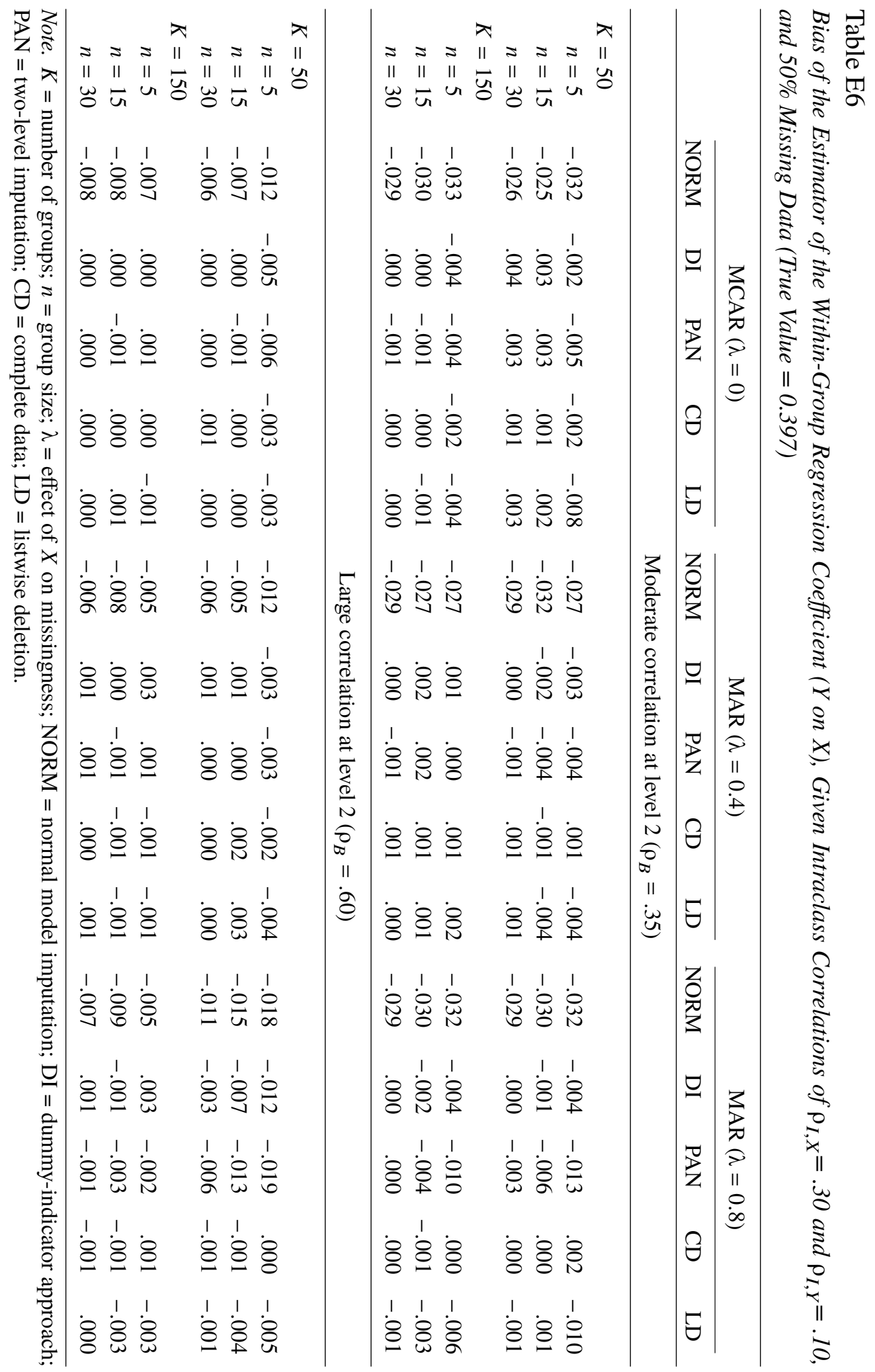




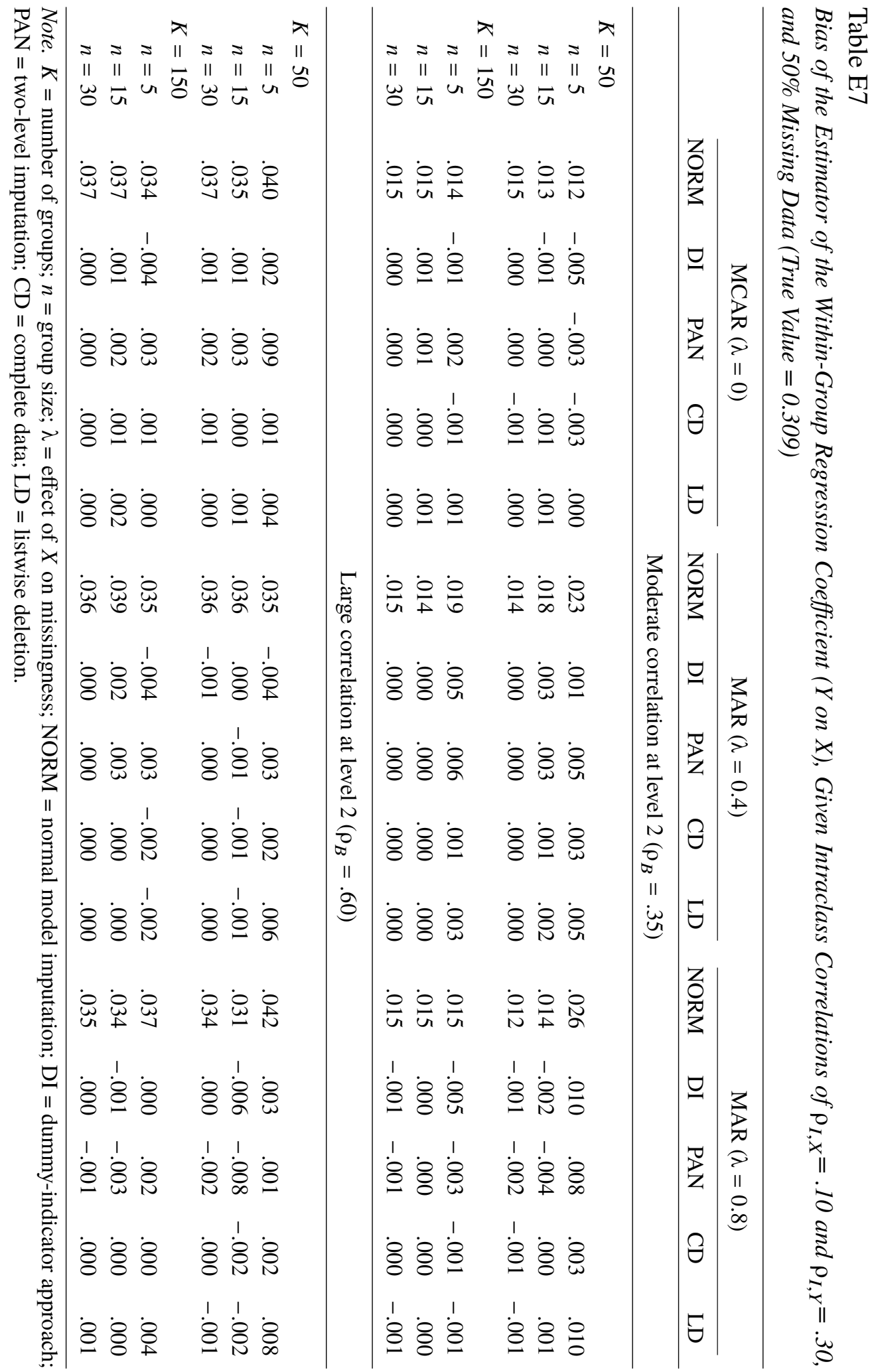




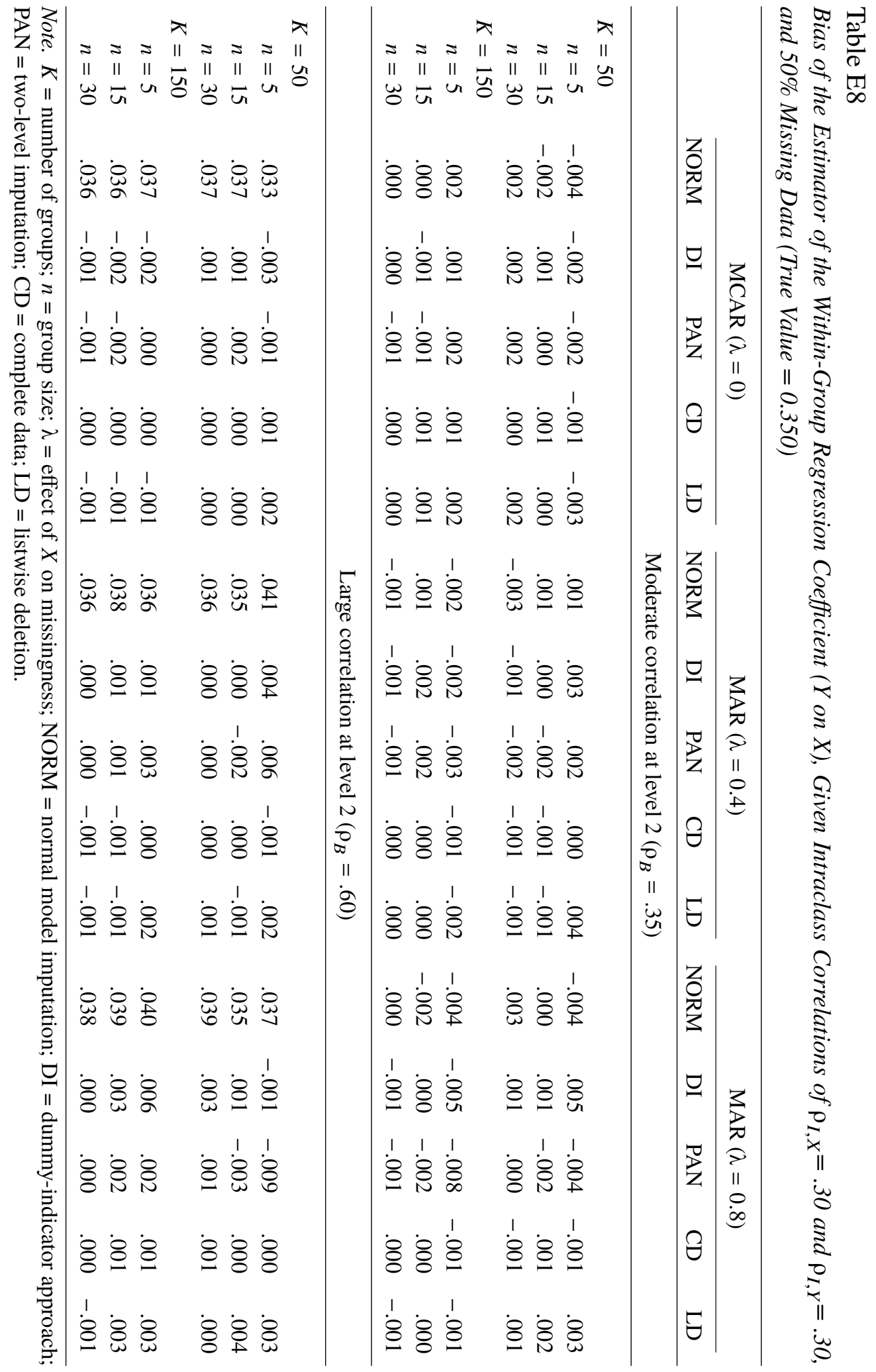




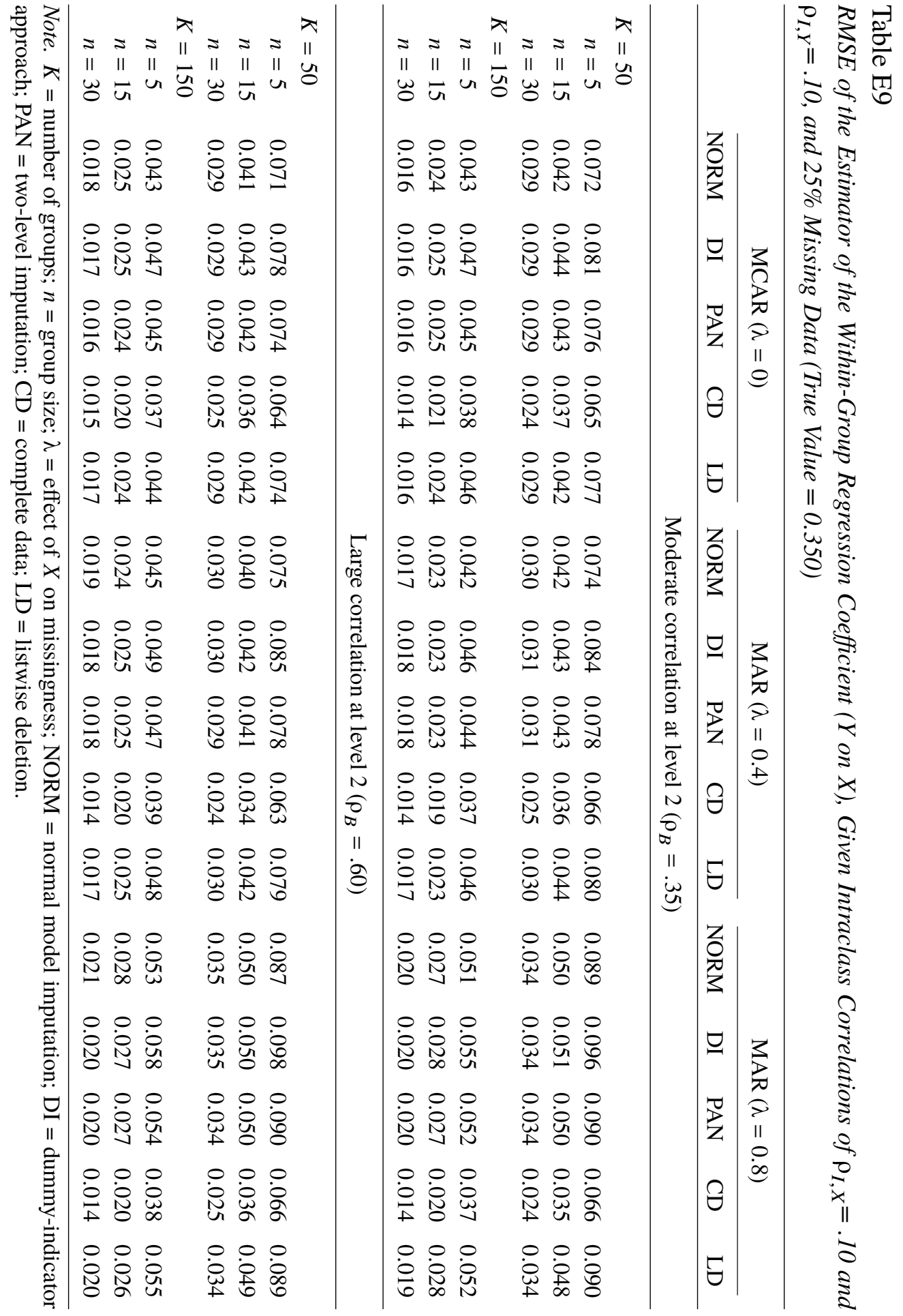




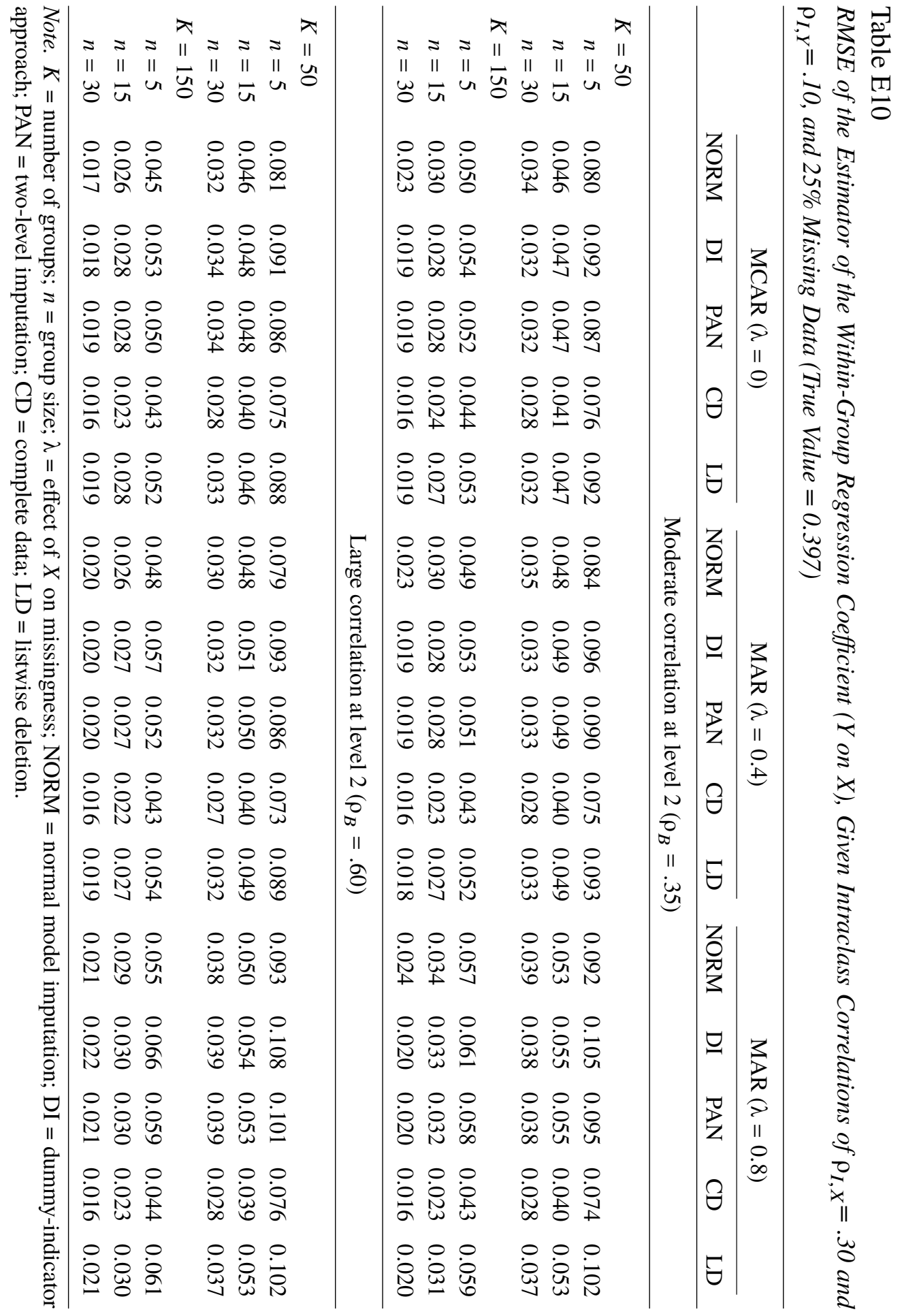




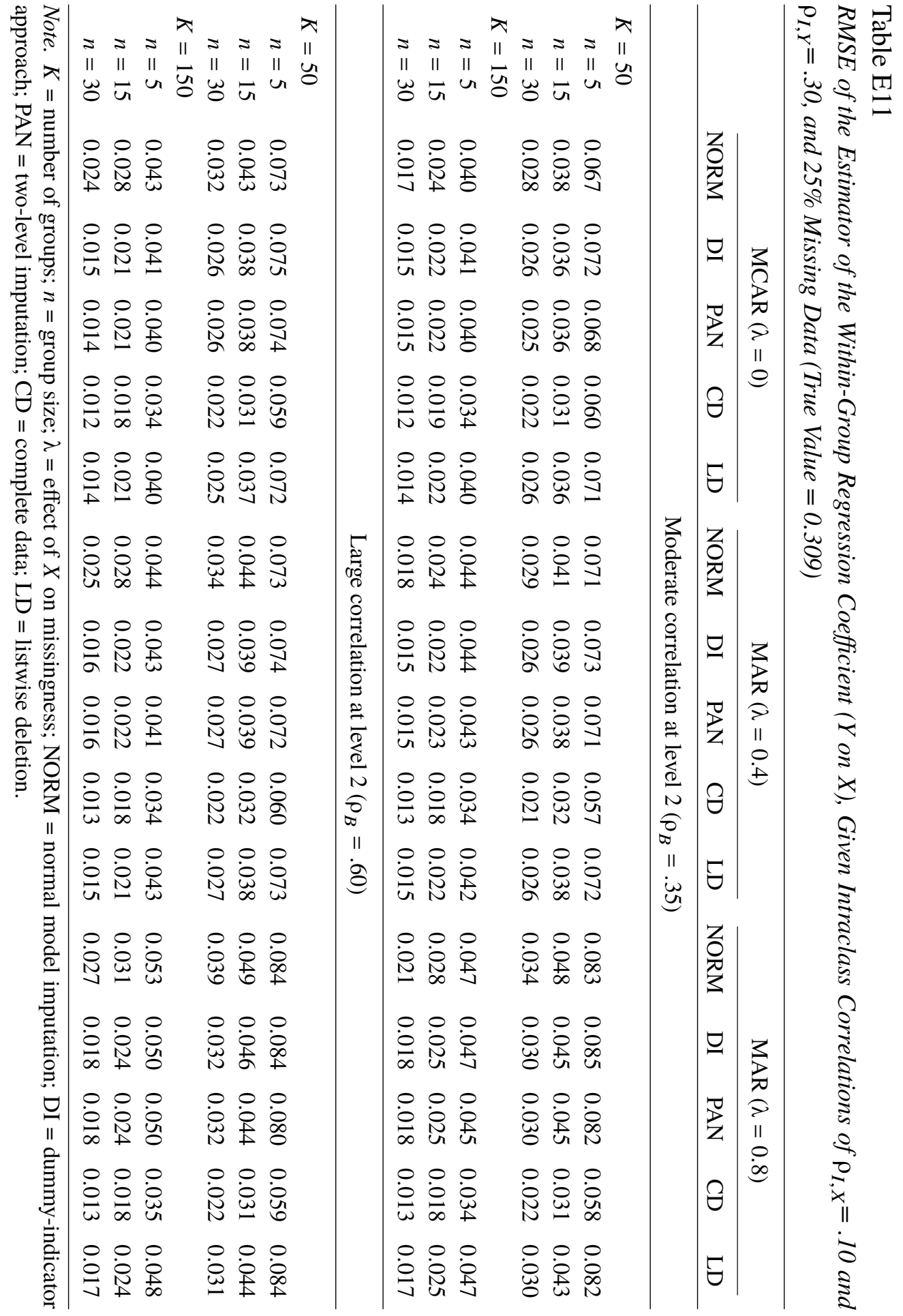




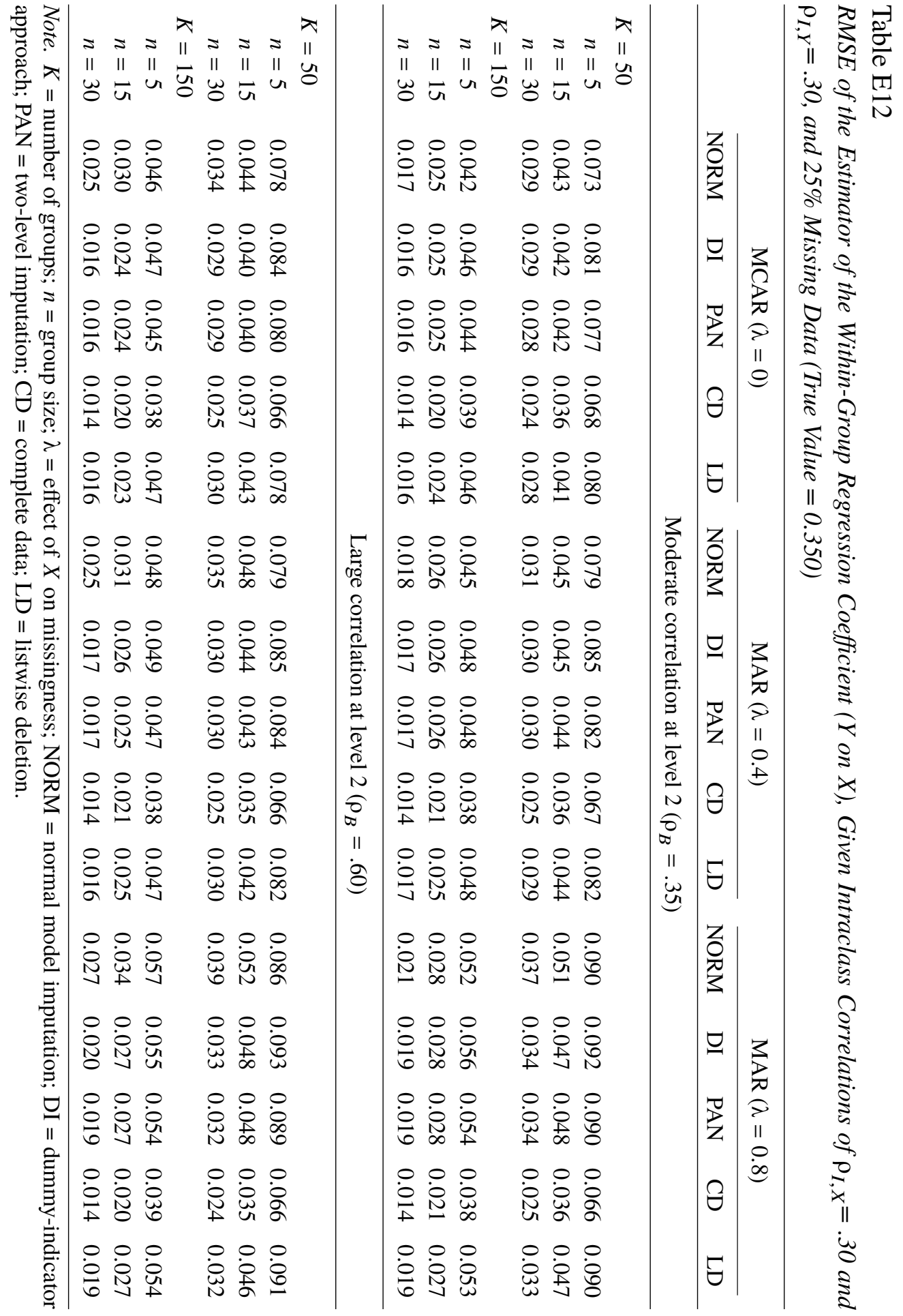




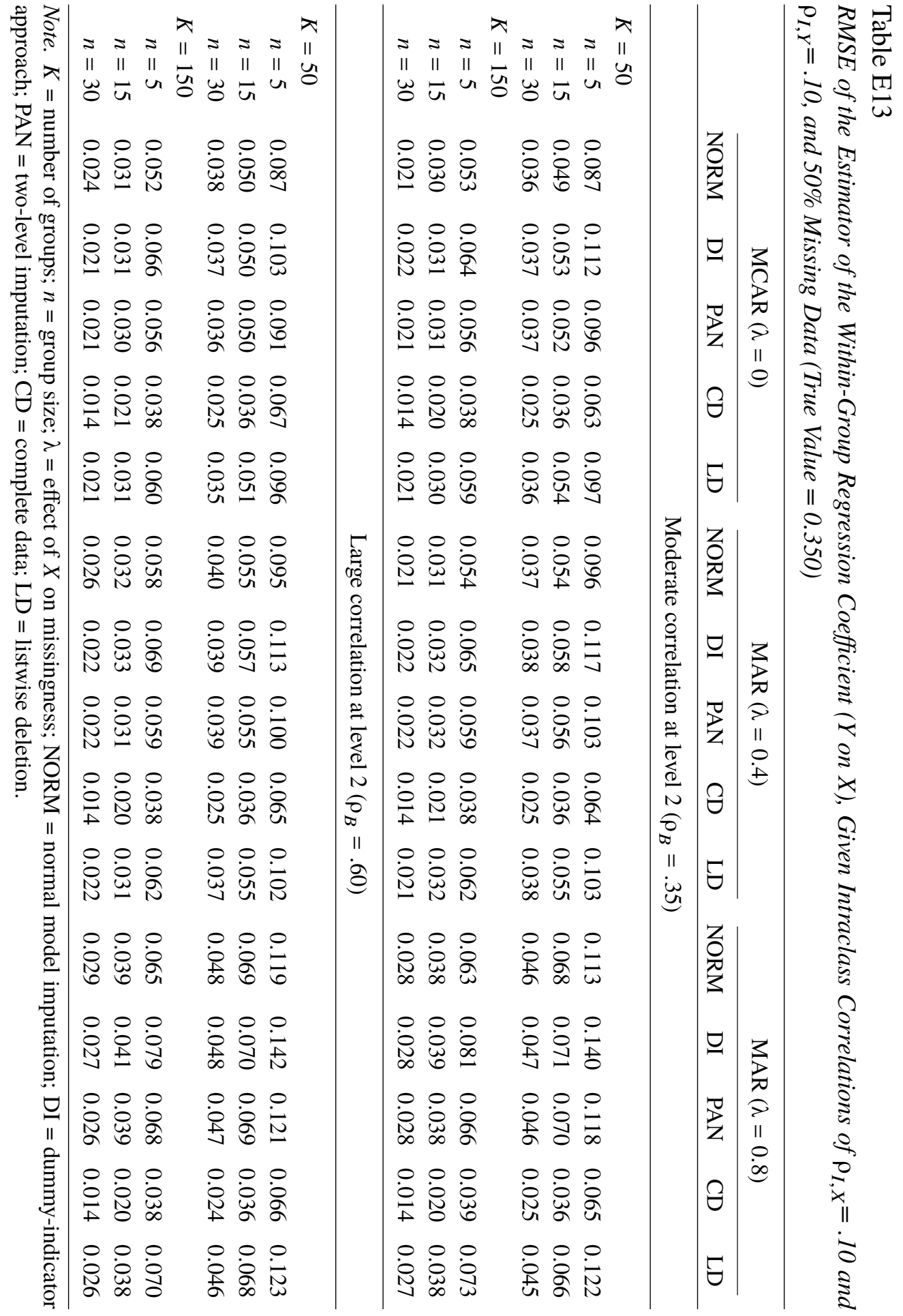




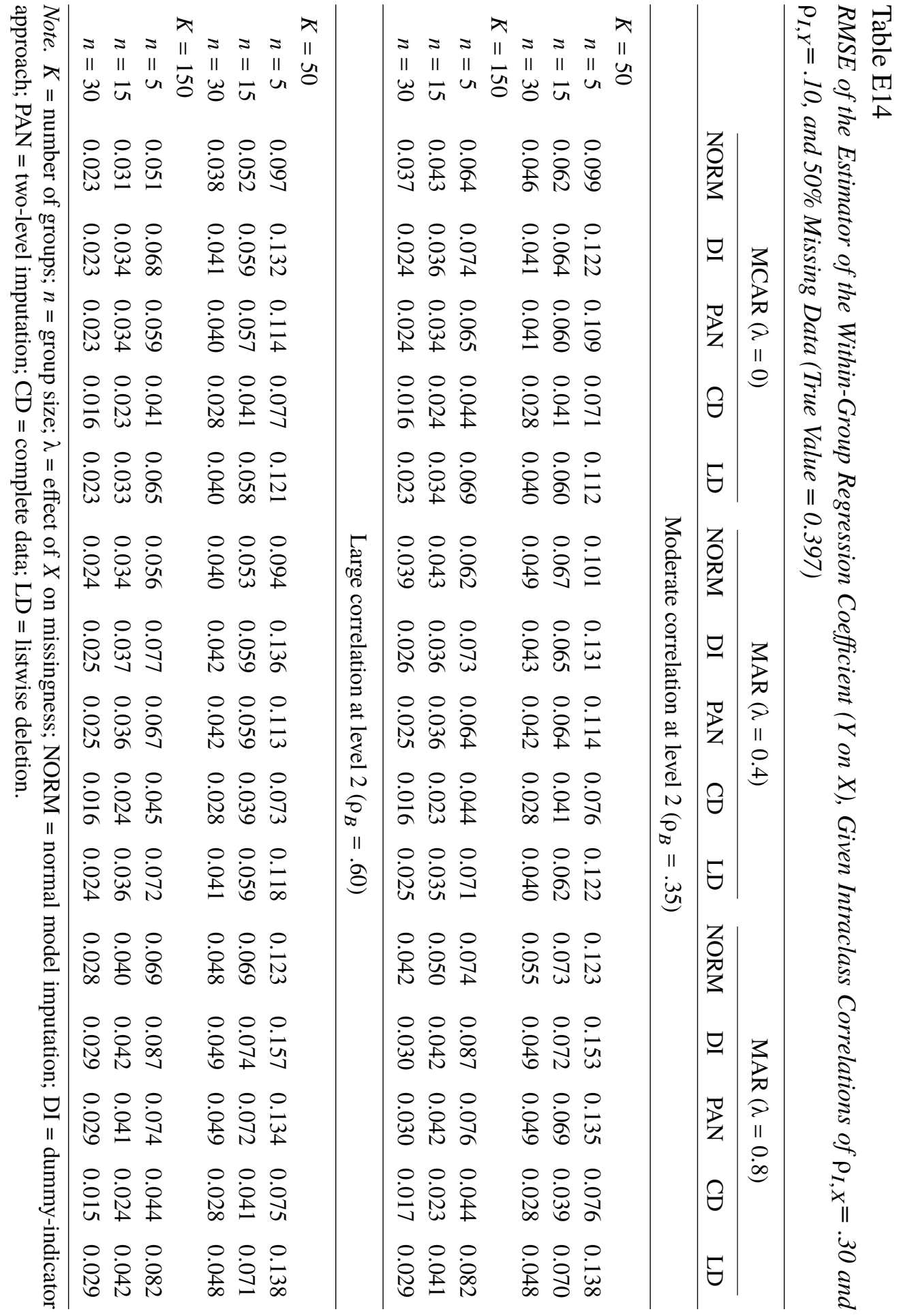




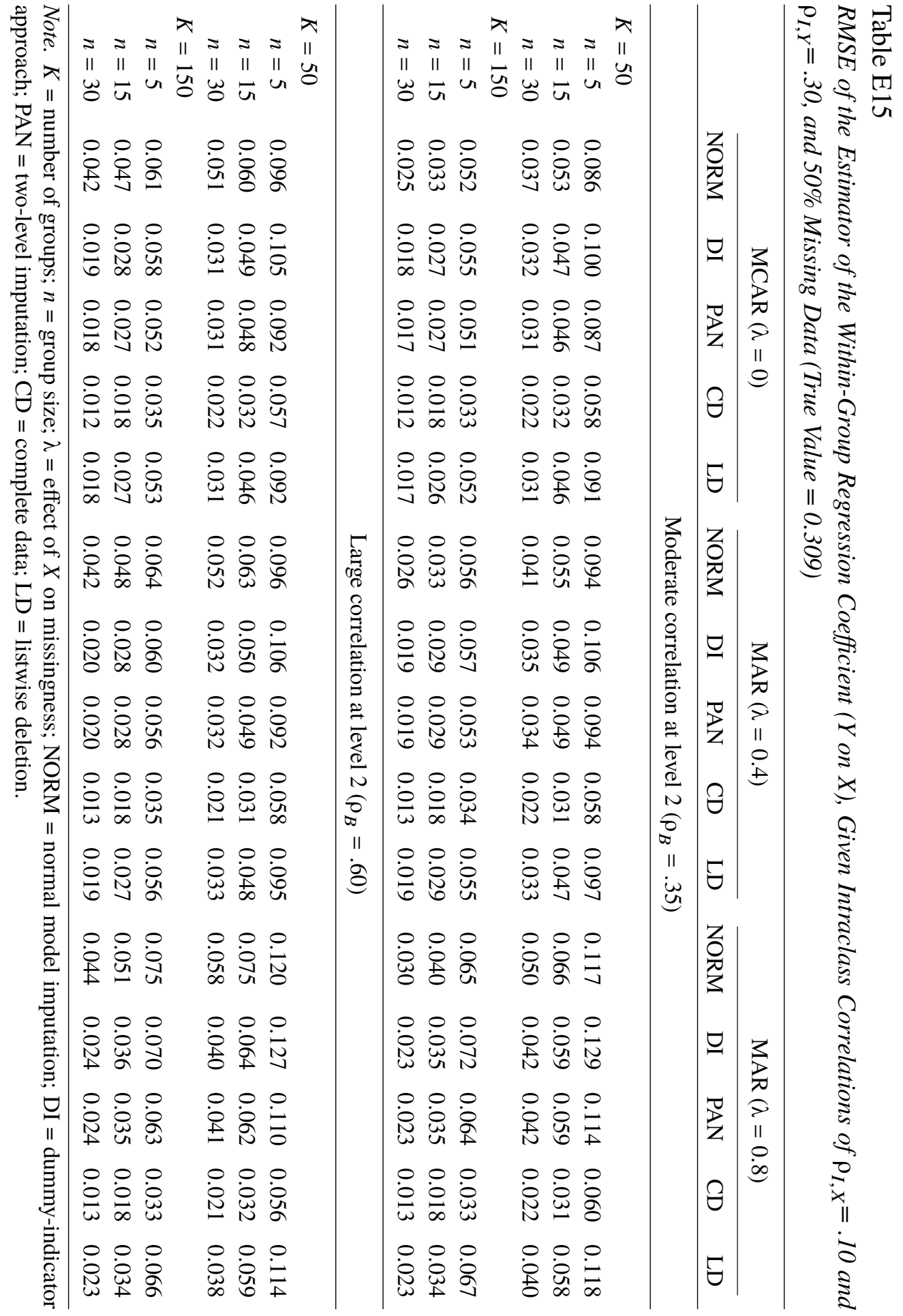




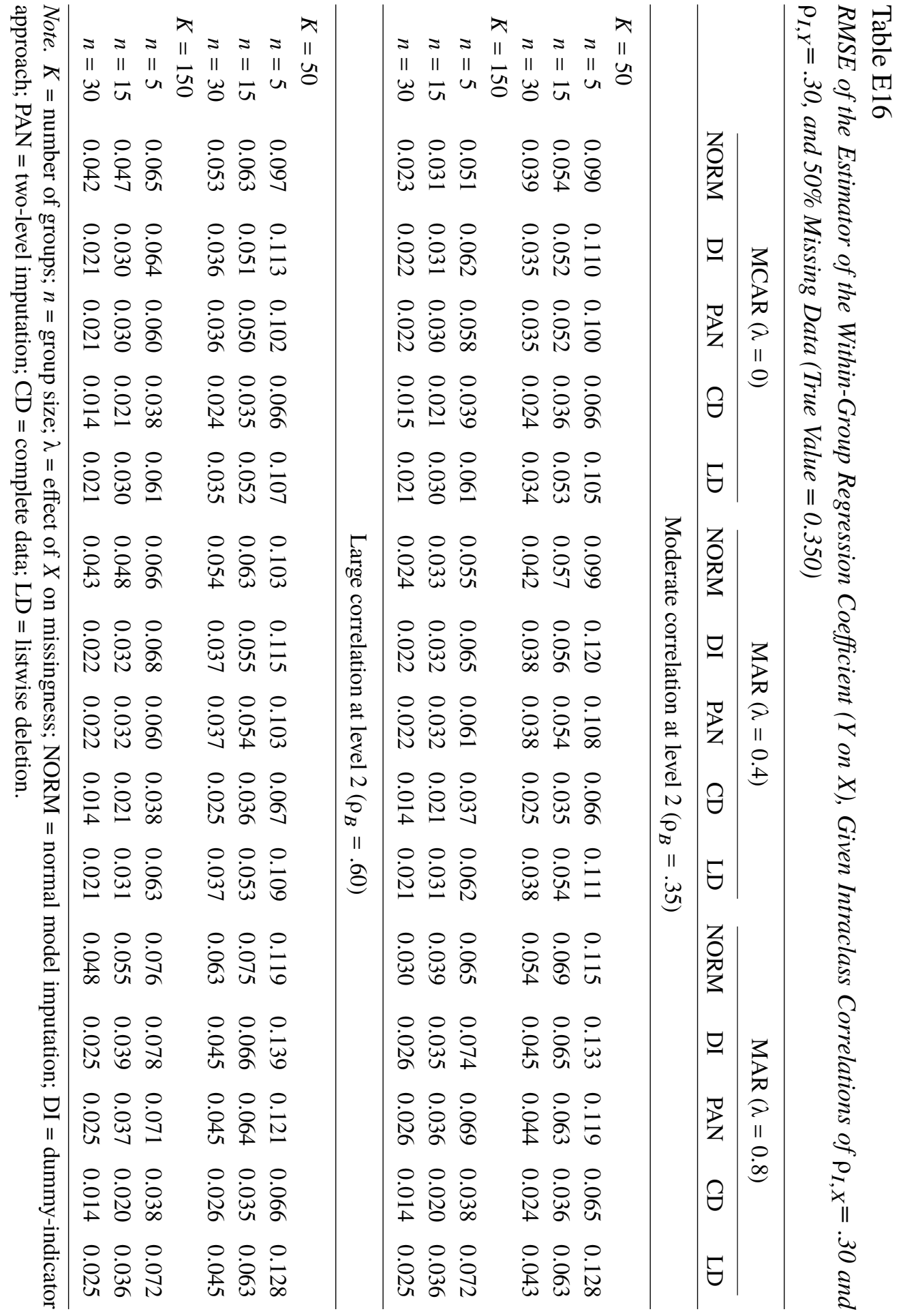




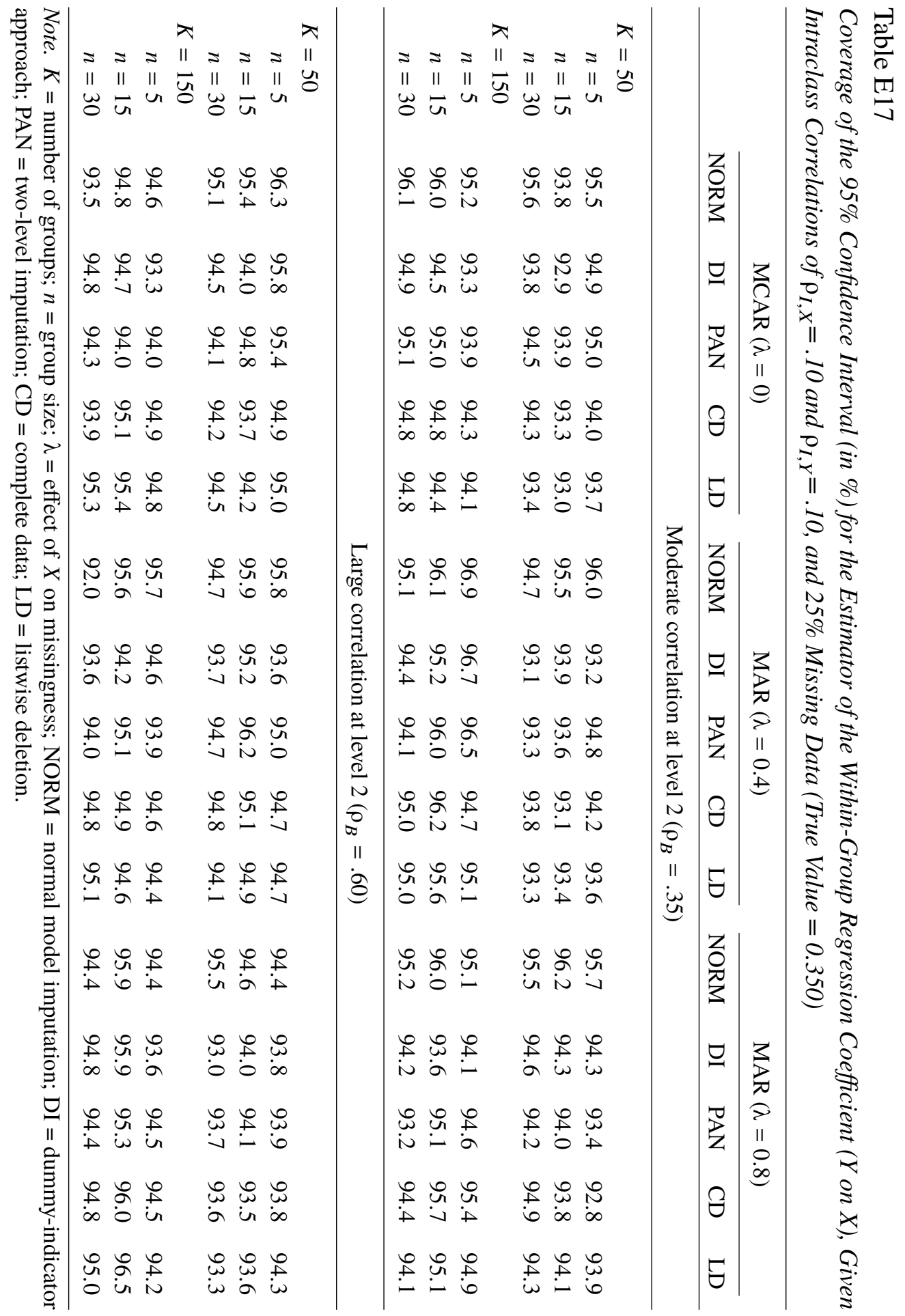




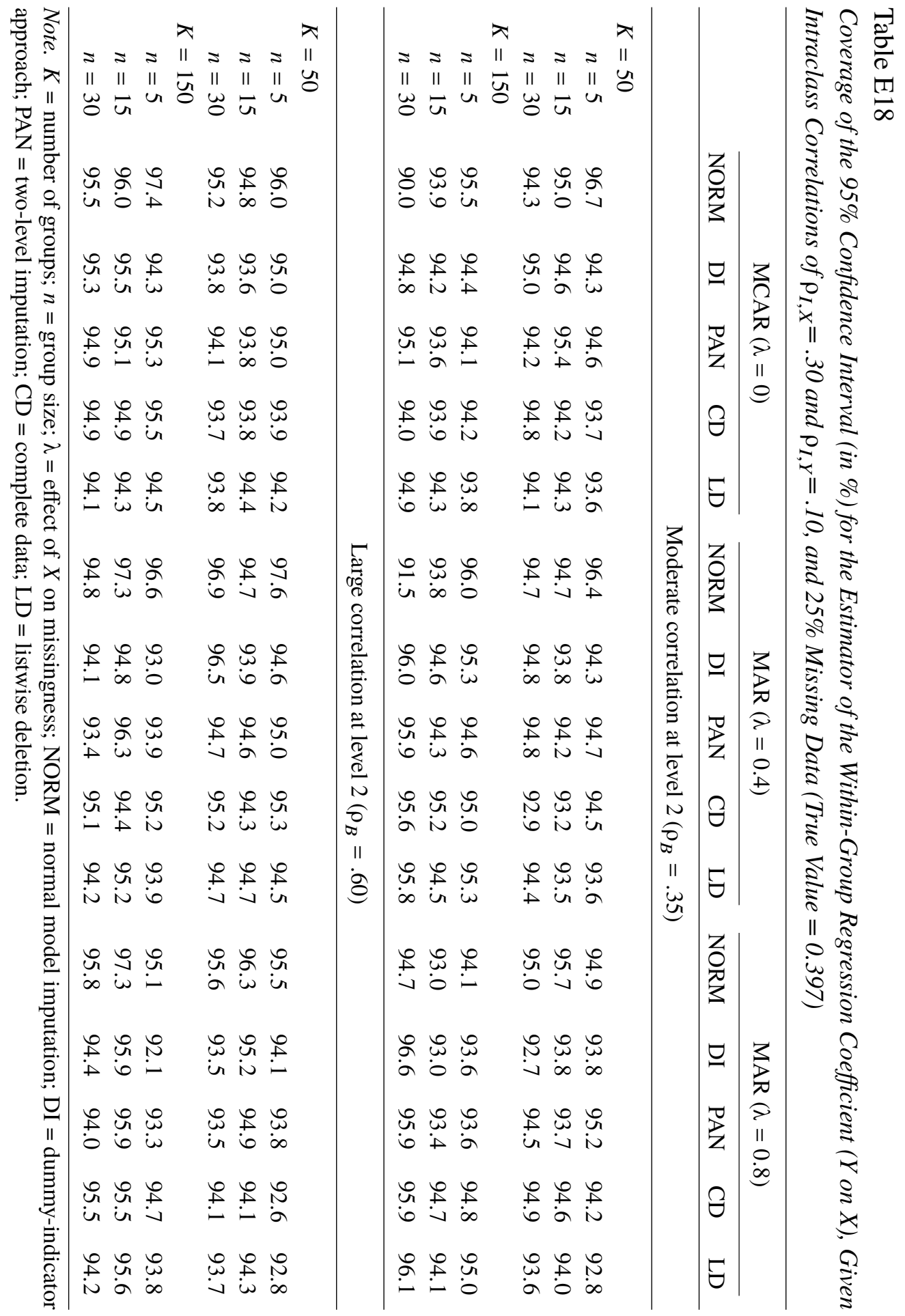




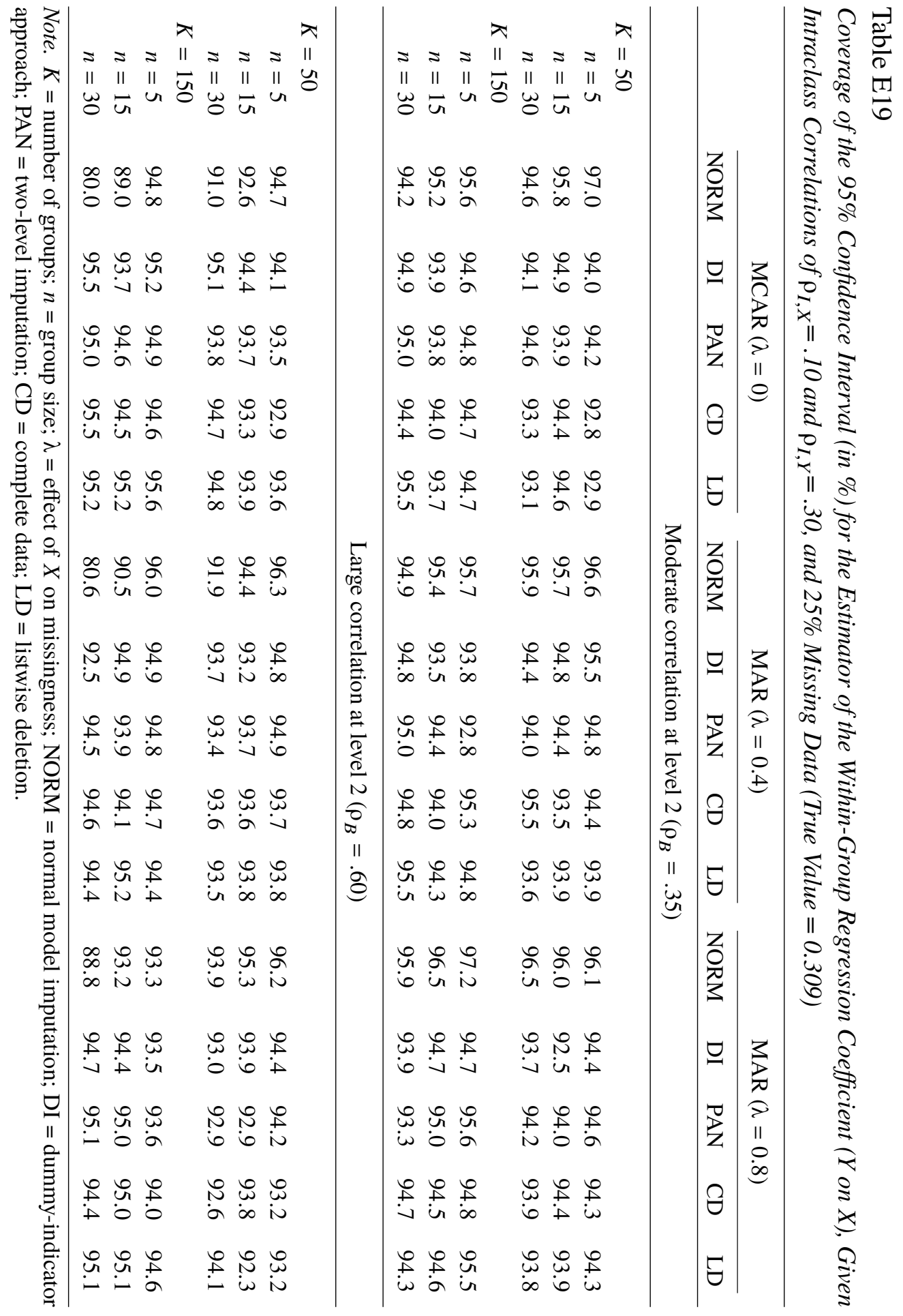




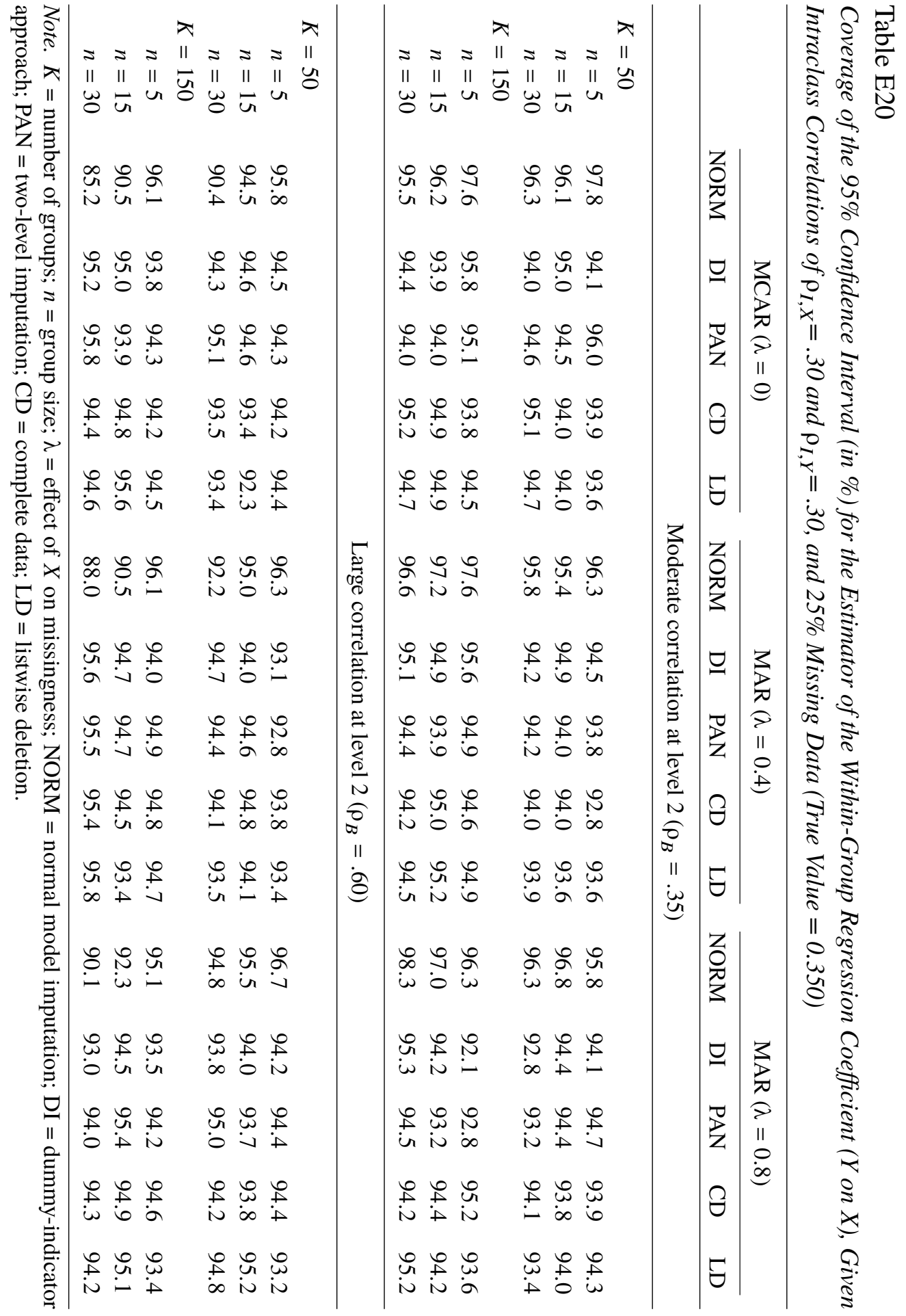




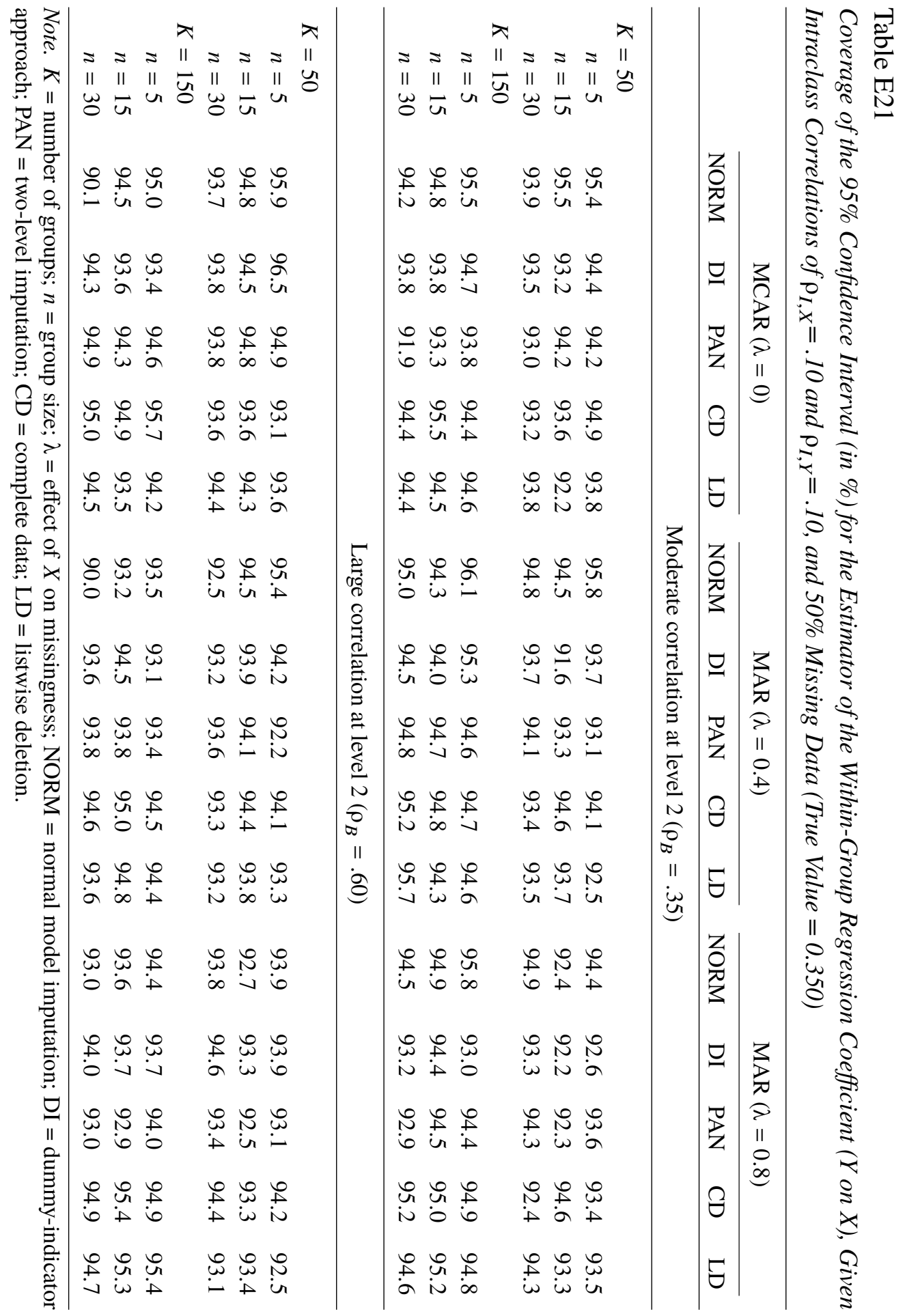




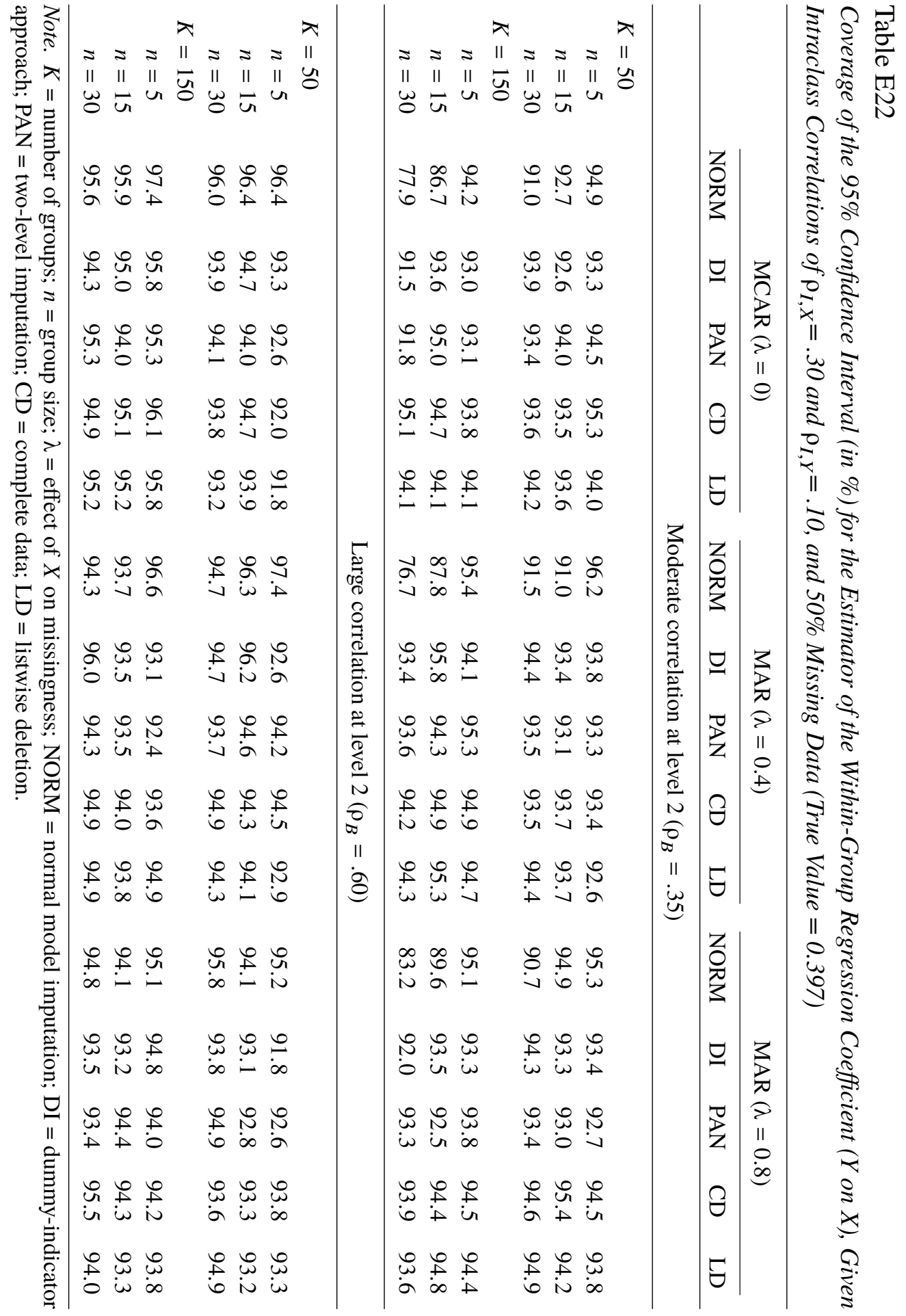




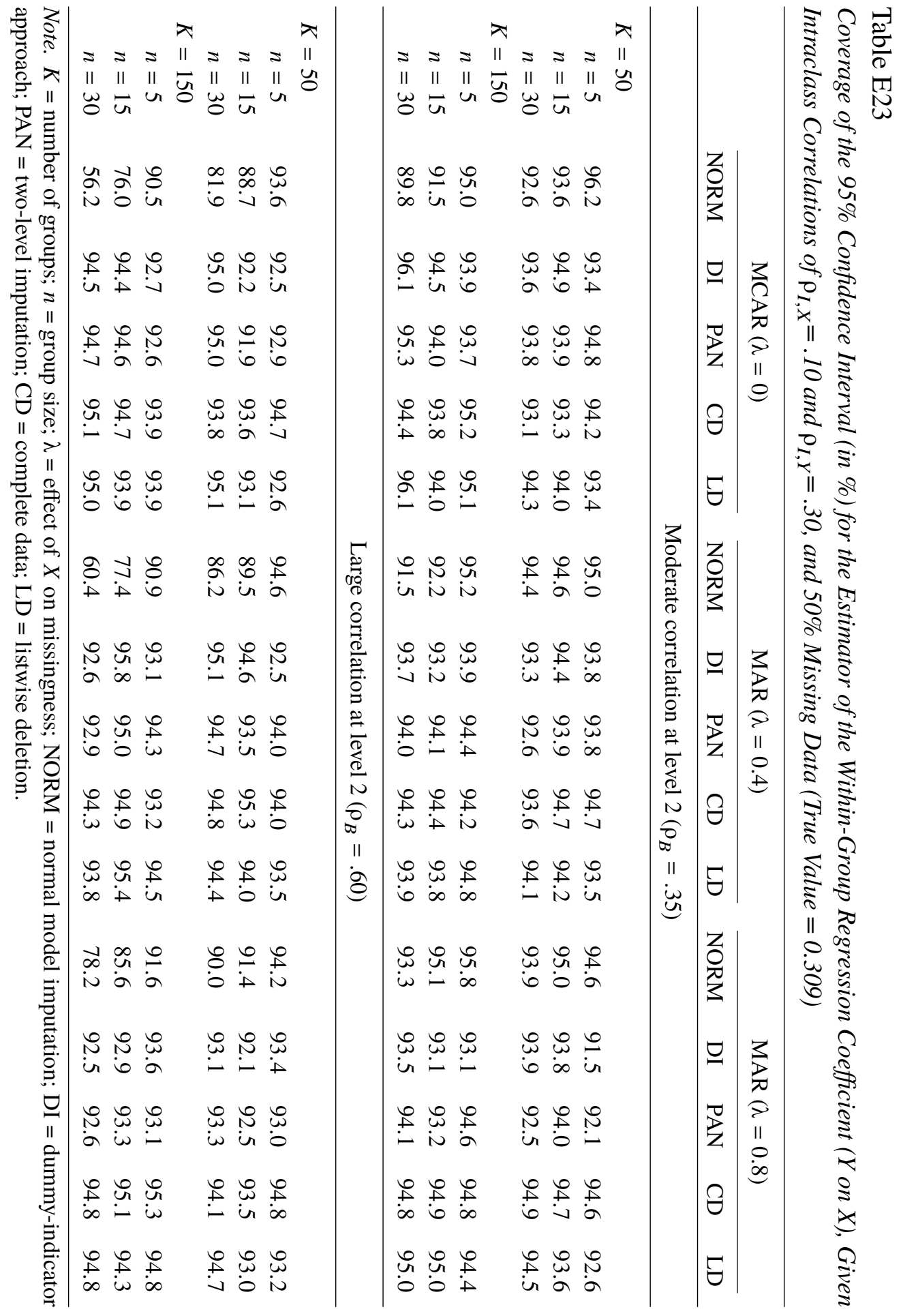




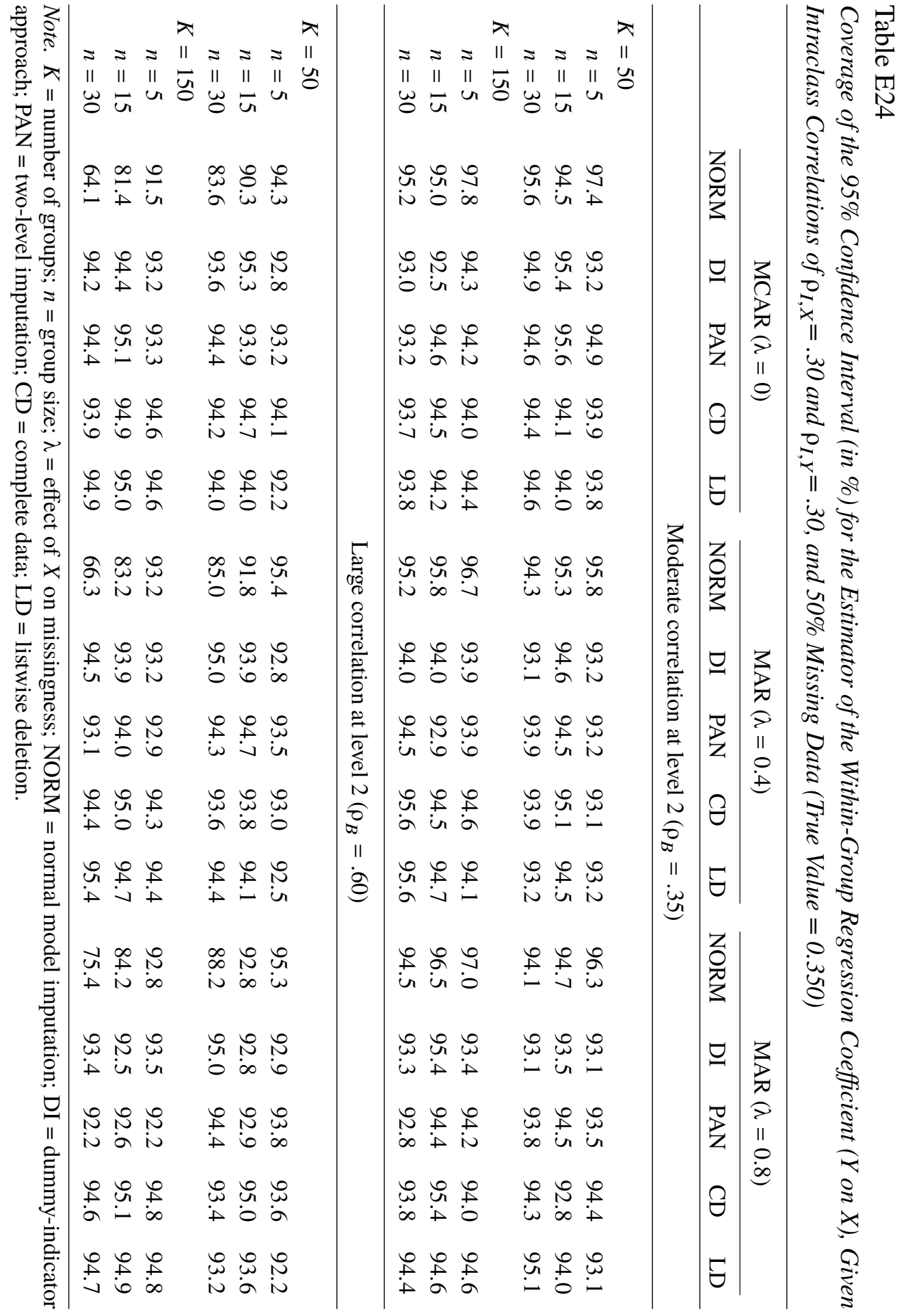




\section{References}

Carpenter, J. R., \& Kenward, M. G. (2013). Multiple imputation and its application. Hoboken, NJ: Wiley.

Enders, C. K., Mistler, S. A., \& Keller, B. T. (in press). Multilevel multiple imputation: A review and evaluation of joint modeling and chained equations imputation. Psychological Methods. doi: 10.1037/met0000063

Grund, S., Lüdtke, O., \& Robitzsch, A. (in press). Multiple imputation of missing covariate values in multilevel models with random slopes: A cautionary note. Behavior Research Methods. doi: 10.3758/s13428-015-0590-3

Lüdtke, O., Marsh, H. W., Robitzsch, A., Trautwein, U., Asparouhov, T., \& Muthén, B. O. (2008). The multilevel latent covariate model: A new, more reliable approach to group-level effects in contextual studies. Psychological Methods, 13, 203-229. doi: $10.1037 / \mathrm{a} 0012869$

Magnus, J. R., \& Neudecker, H. (1988). Matrix differential calculus with applications in statistics and econometrics. Hoboken, NJ: Wiley.

Mehta, P. D., \& Neale, M. C. (2005). People are variables too: Multilevel structural equations modeling. Psychological Methods, 10, 259-284. doi: 10.1037/1082-989X .10 .3 .259

Mistler, S. A. (2015). Multilevel multiple imputation: An examination of competing methods (Doctoral dissertation). Arizona State University.

Preacher, K. J., Zhang, Z., \& Zyphur, M. J. (in press). Multilevel structural equation models for assessing moderation within and across levels of analysis. Psychological Methods. doi: $10.1037 /$ met0000052

Raudenbush, S. W., \& Bryk, A. S. (2002). Hierarchical linear models: Applications and data analysis methods (2nd ed.). Thousand Oaks, CA: Sage.

van Buuren, S., \& Groothuis-Oudshoorn, K. (2011). MICE: Multivariate imputation by 
chained equations in R. Journal of Statistical Software, 45(3), 1-67. 